SECTION I

\title{
Animal Viruses, Including Protozoal Viruses
}




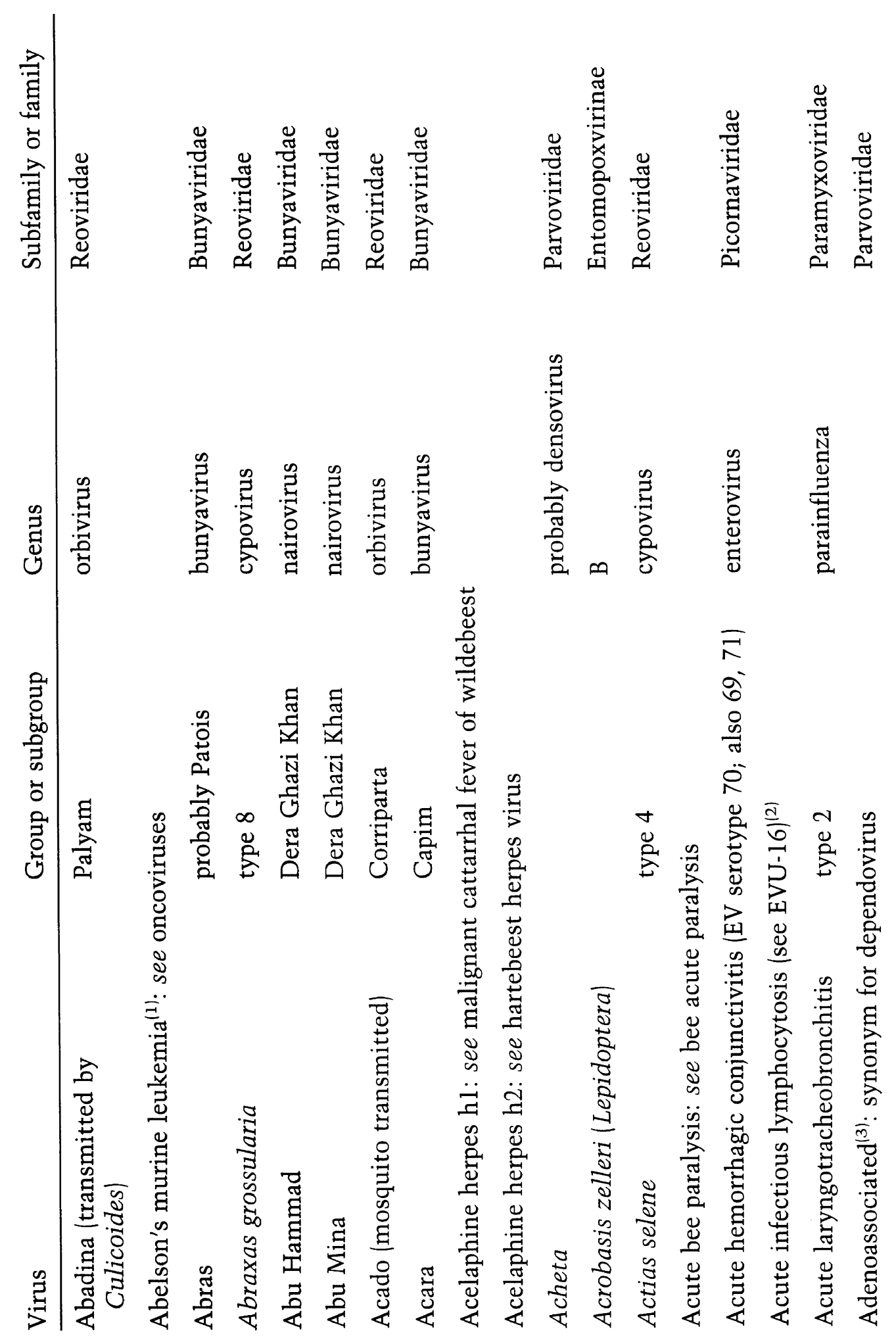



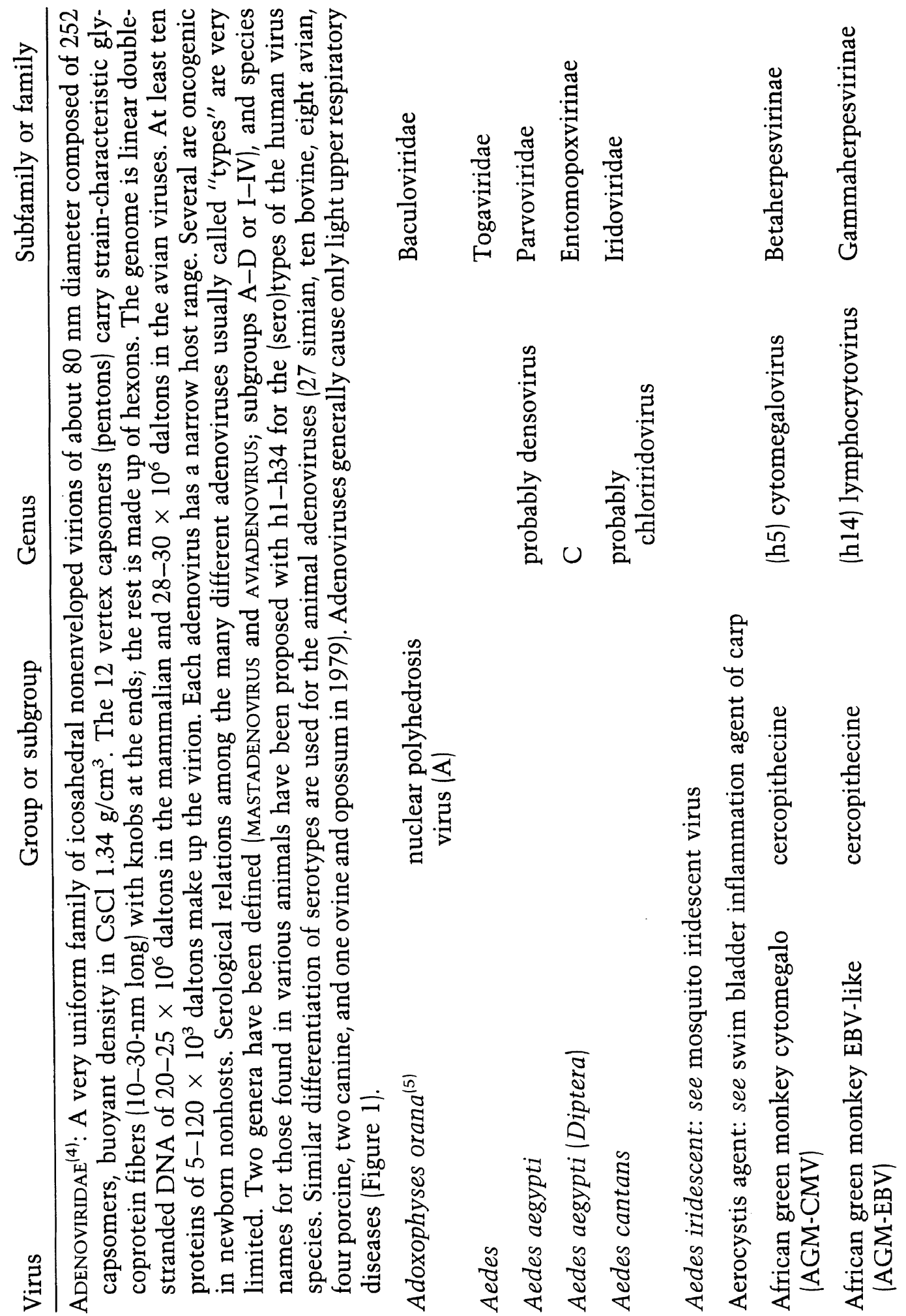

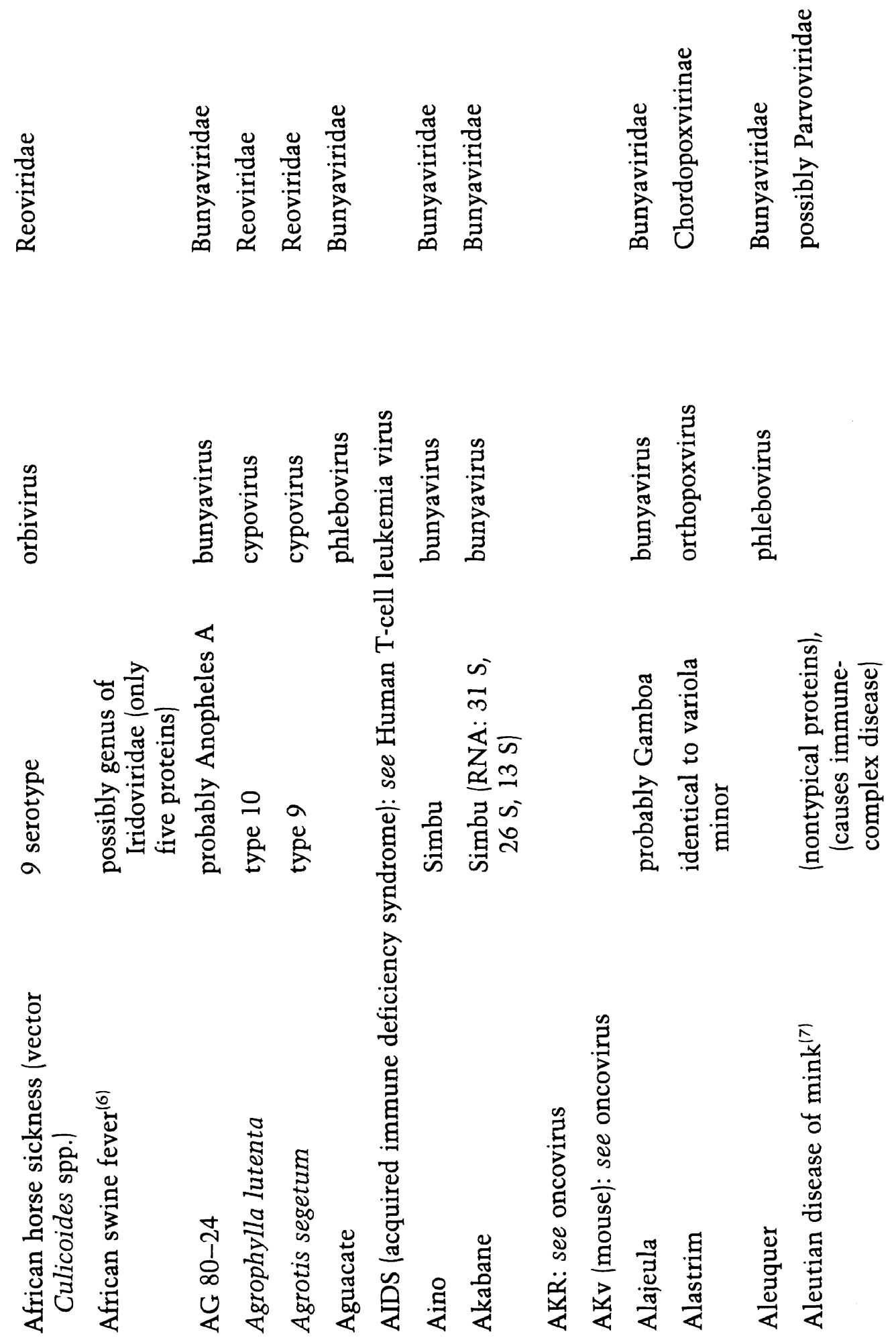

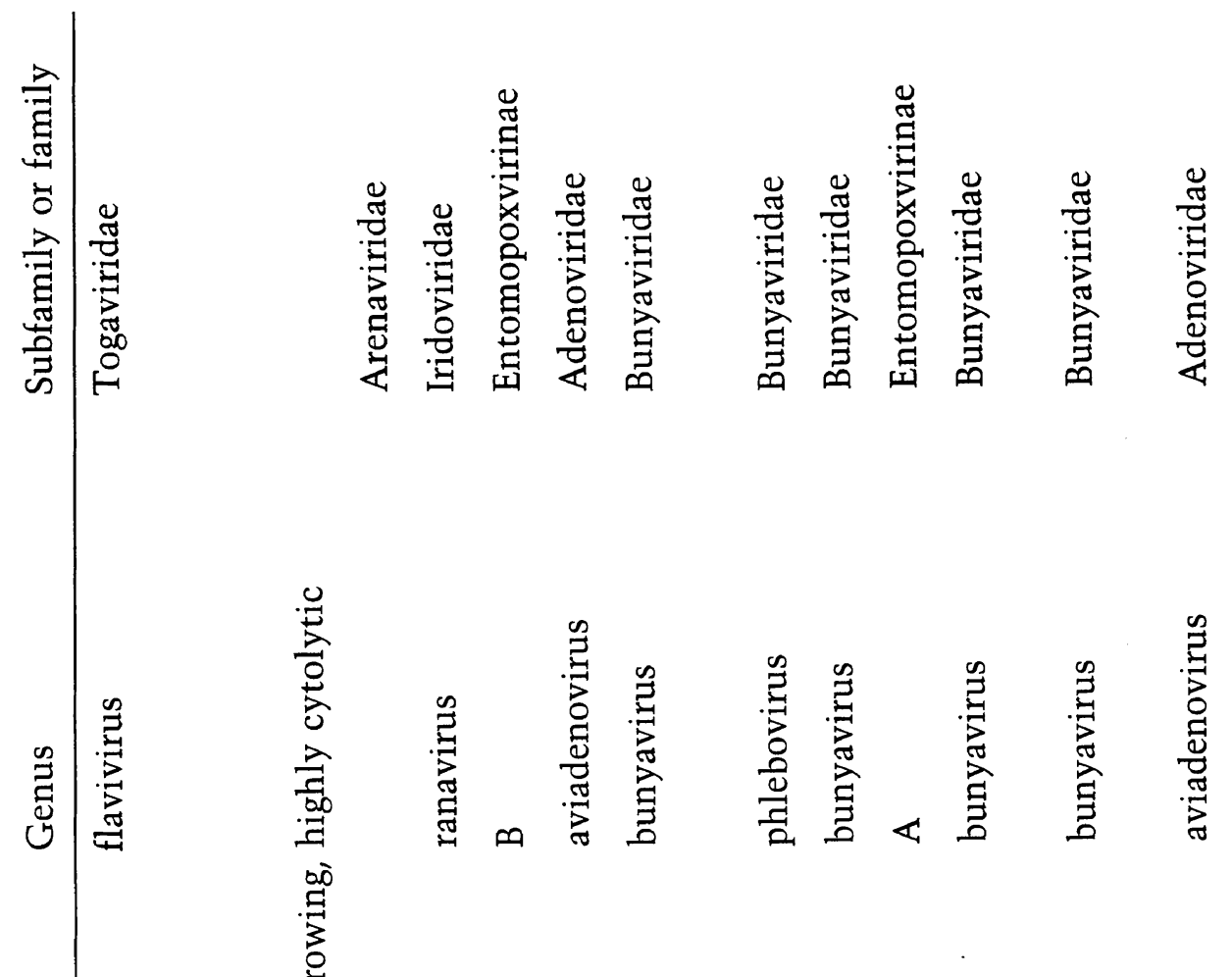

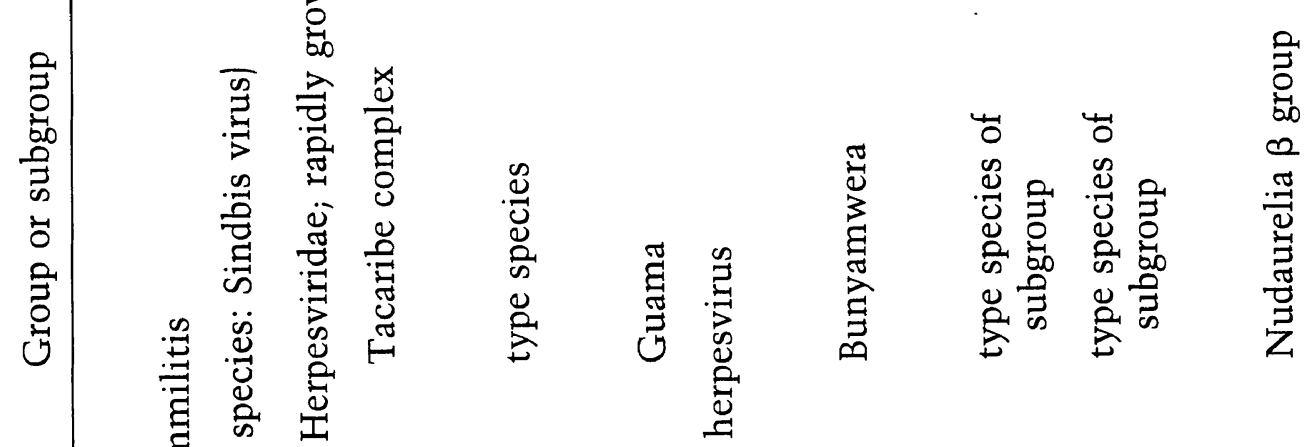

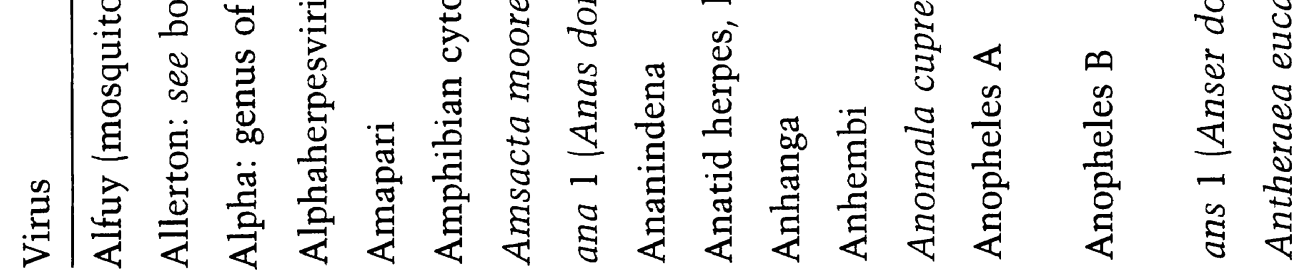




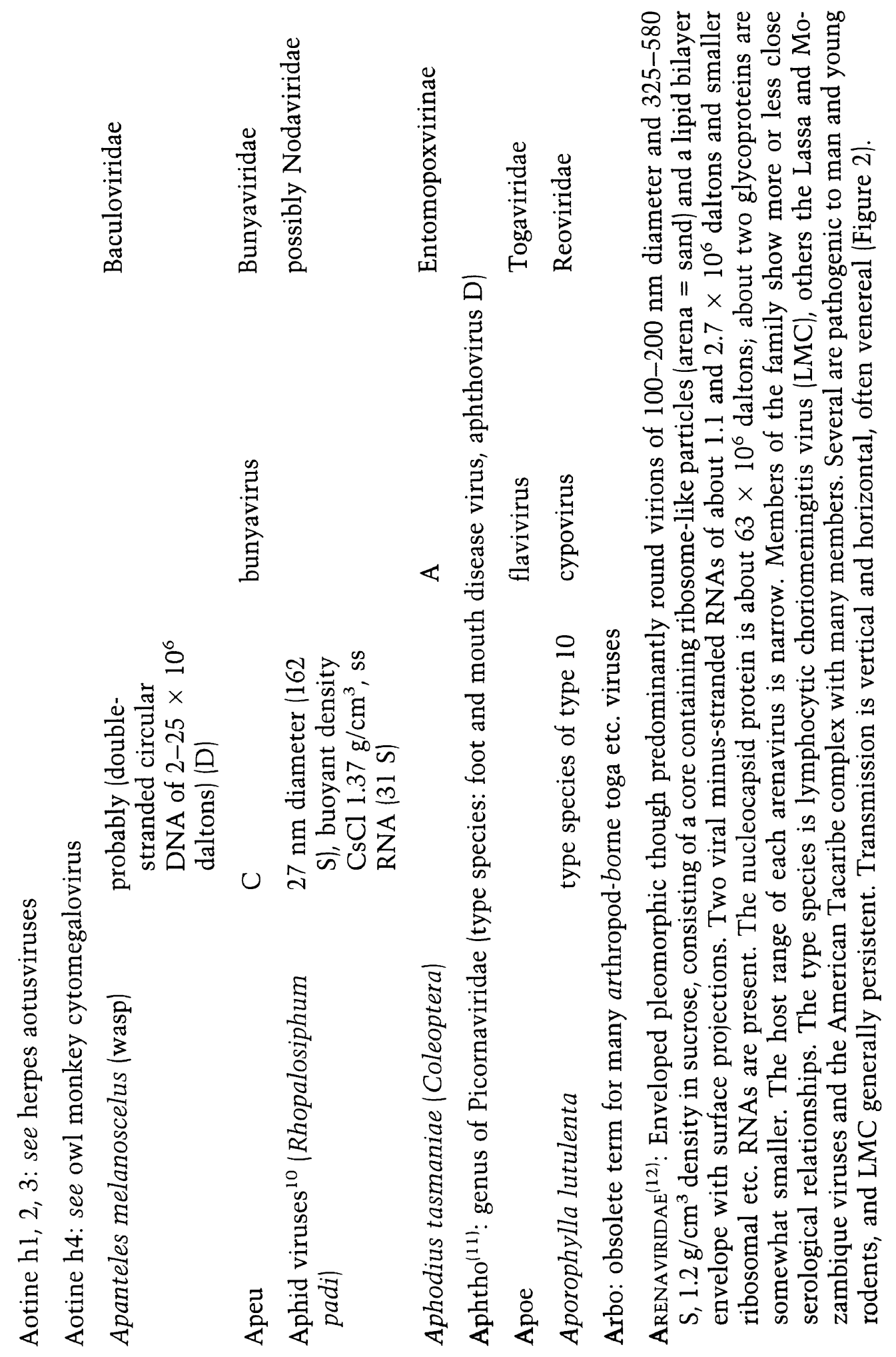




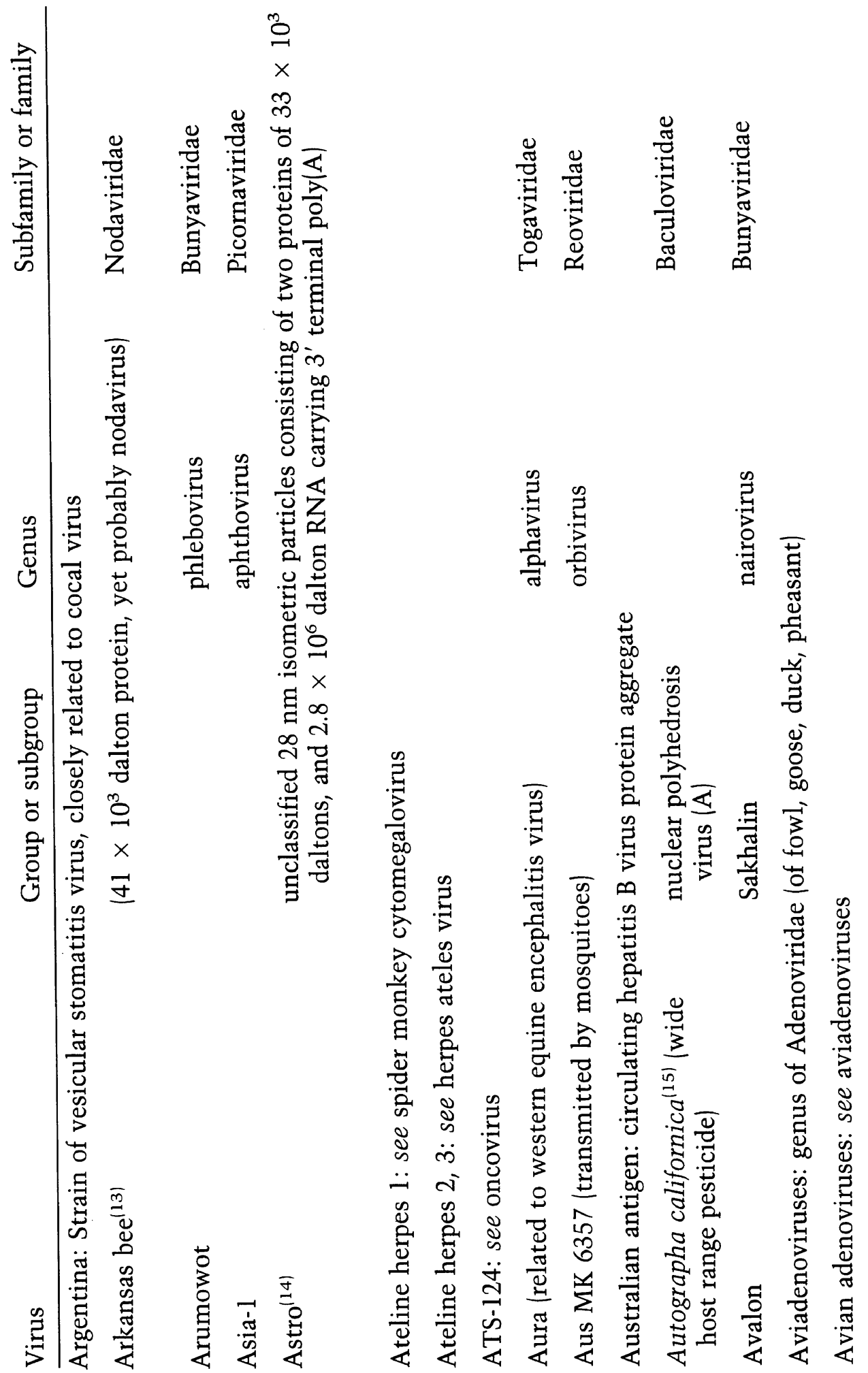




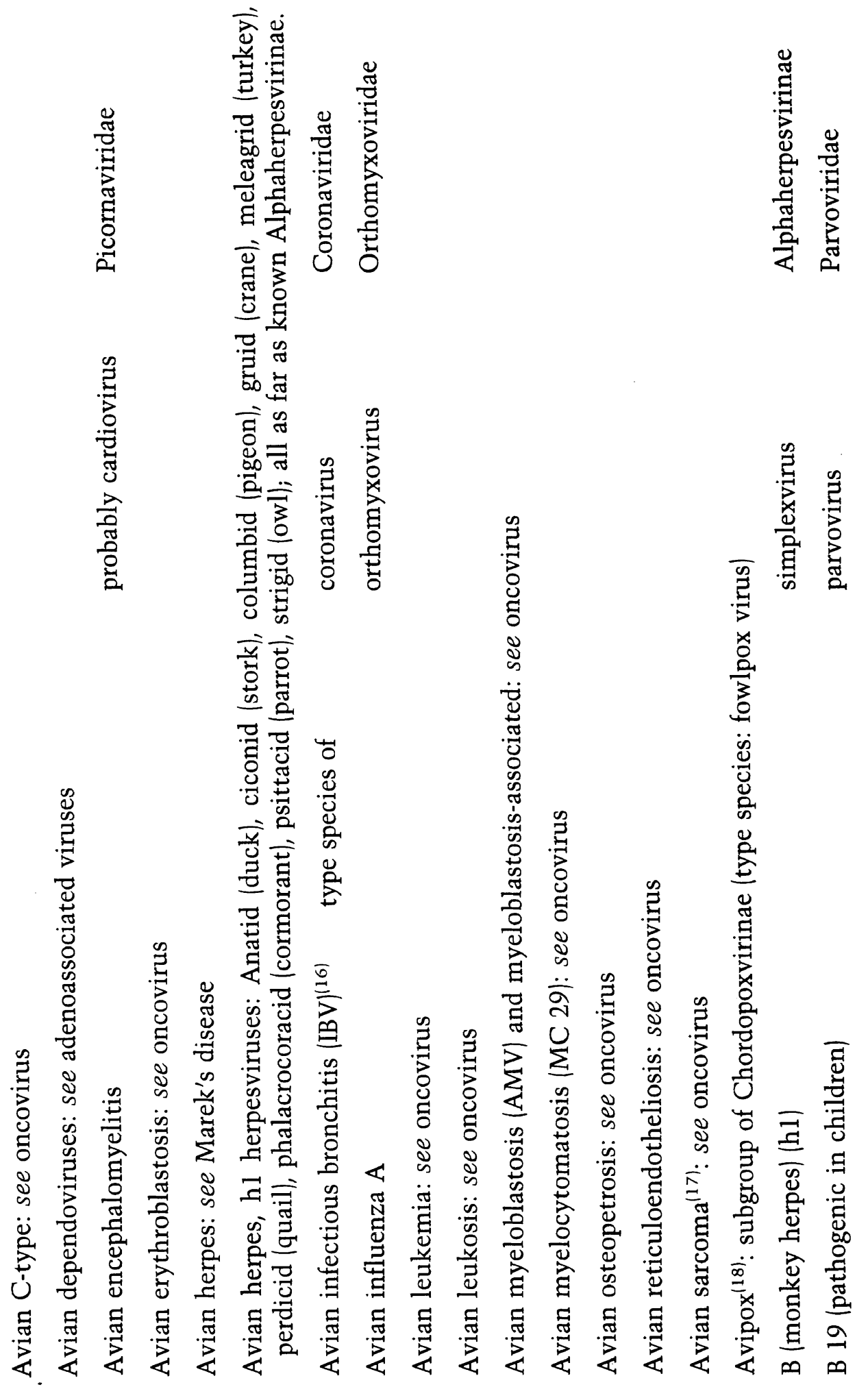




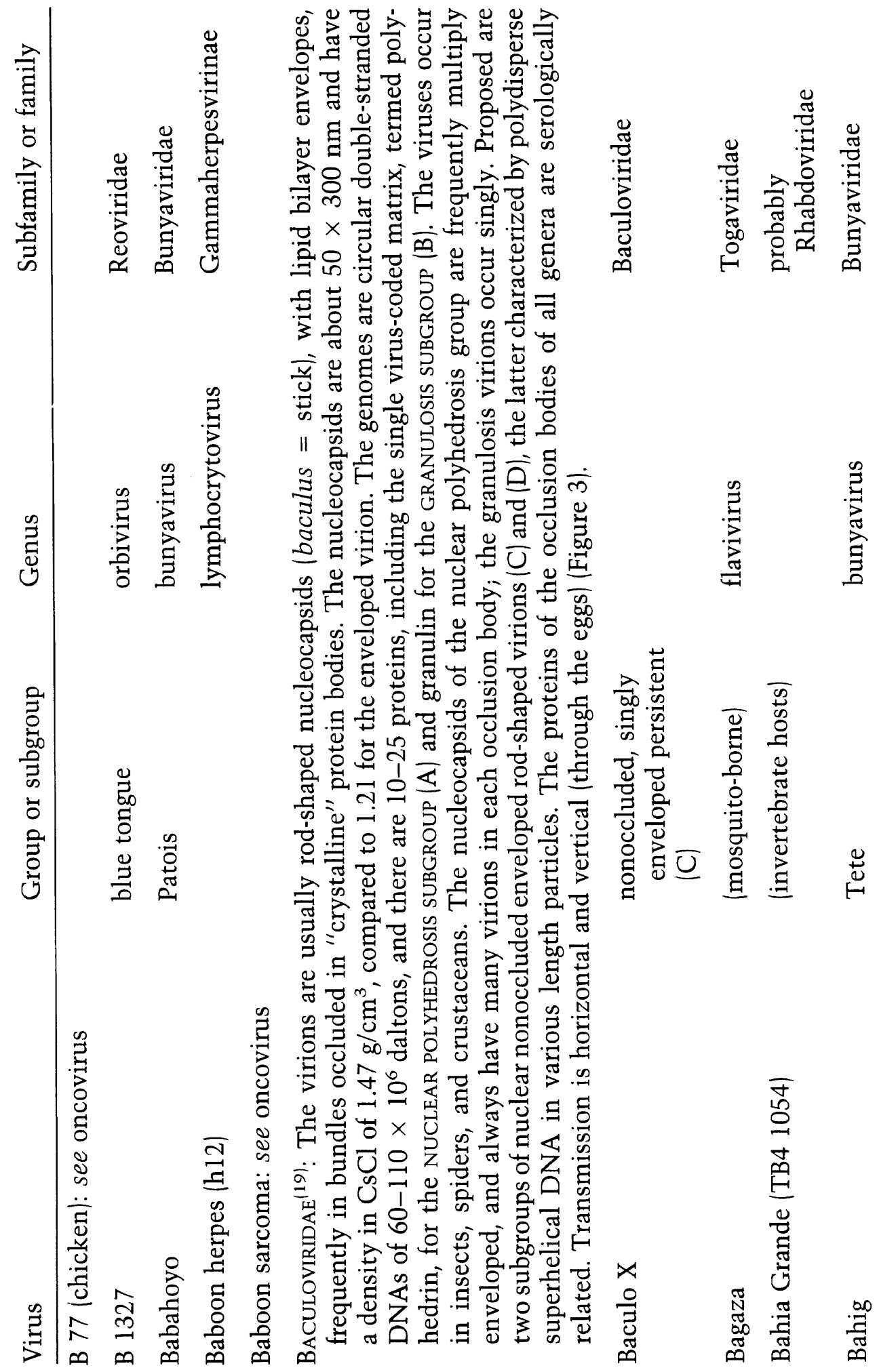




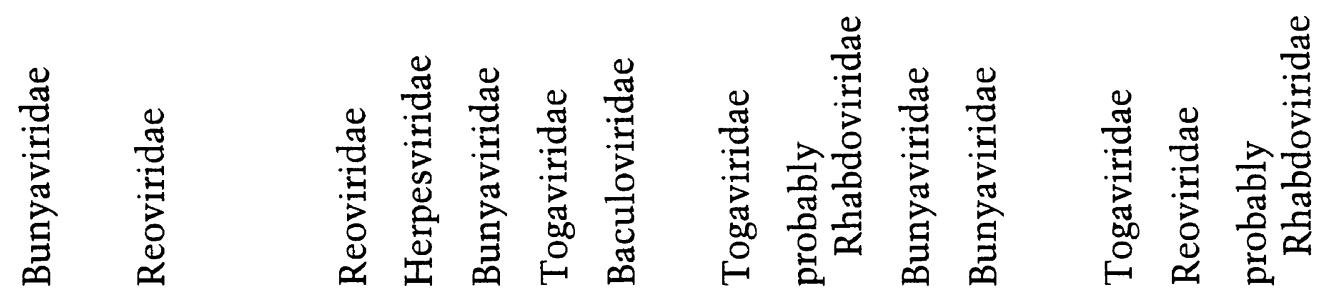

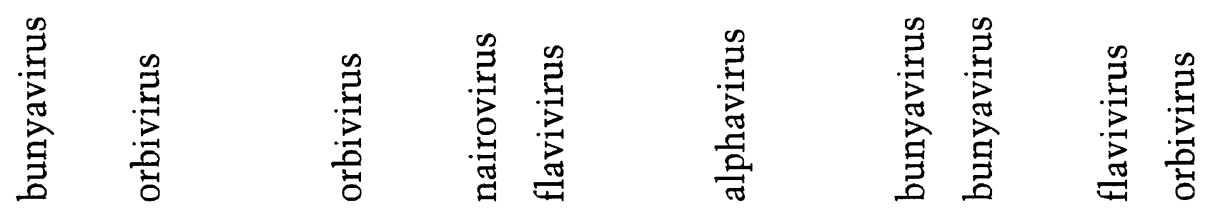
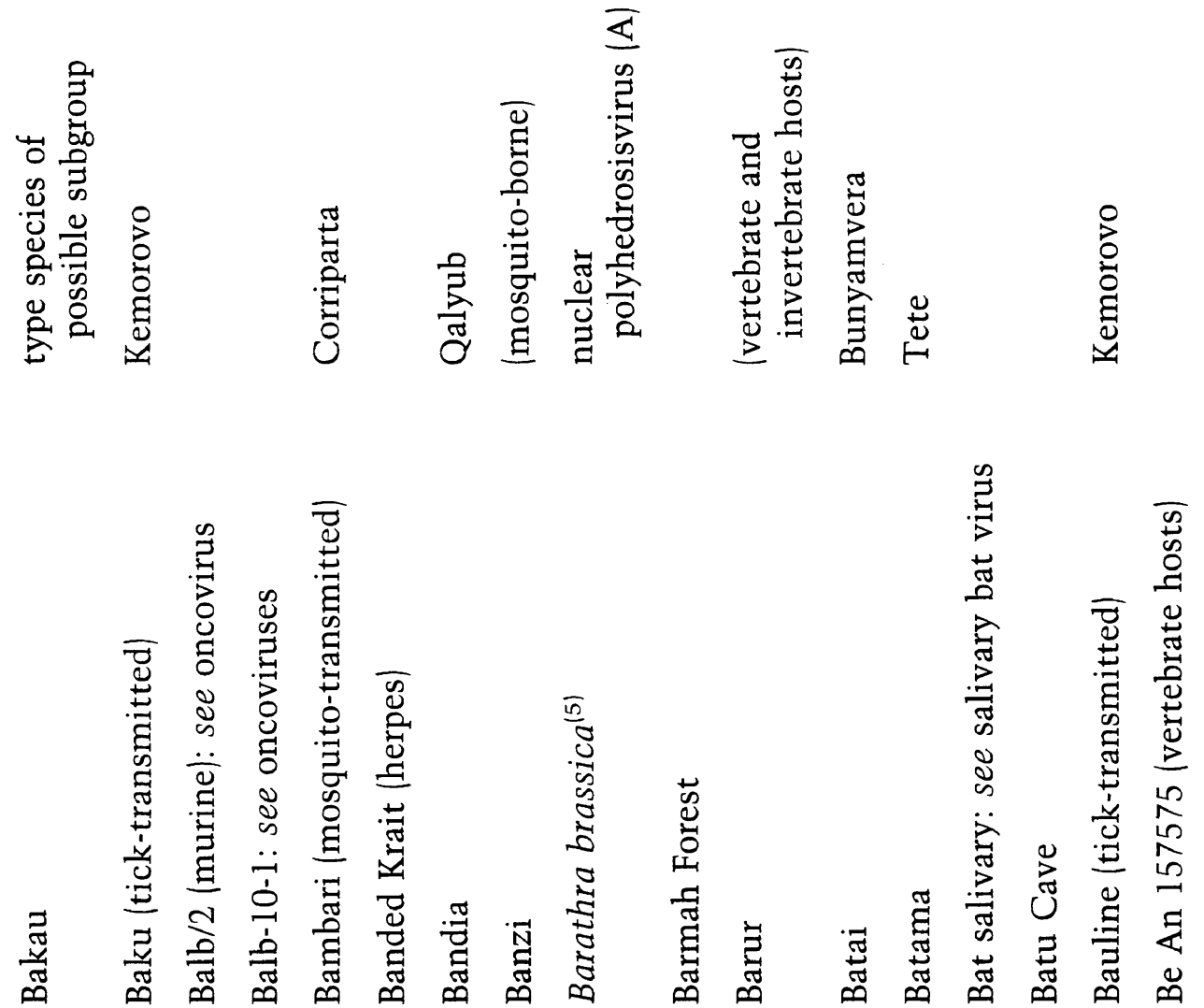


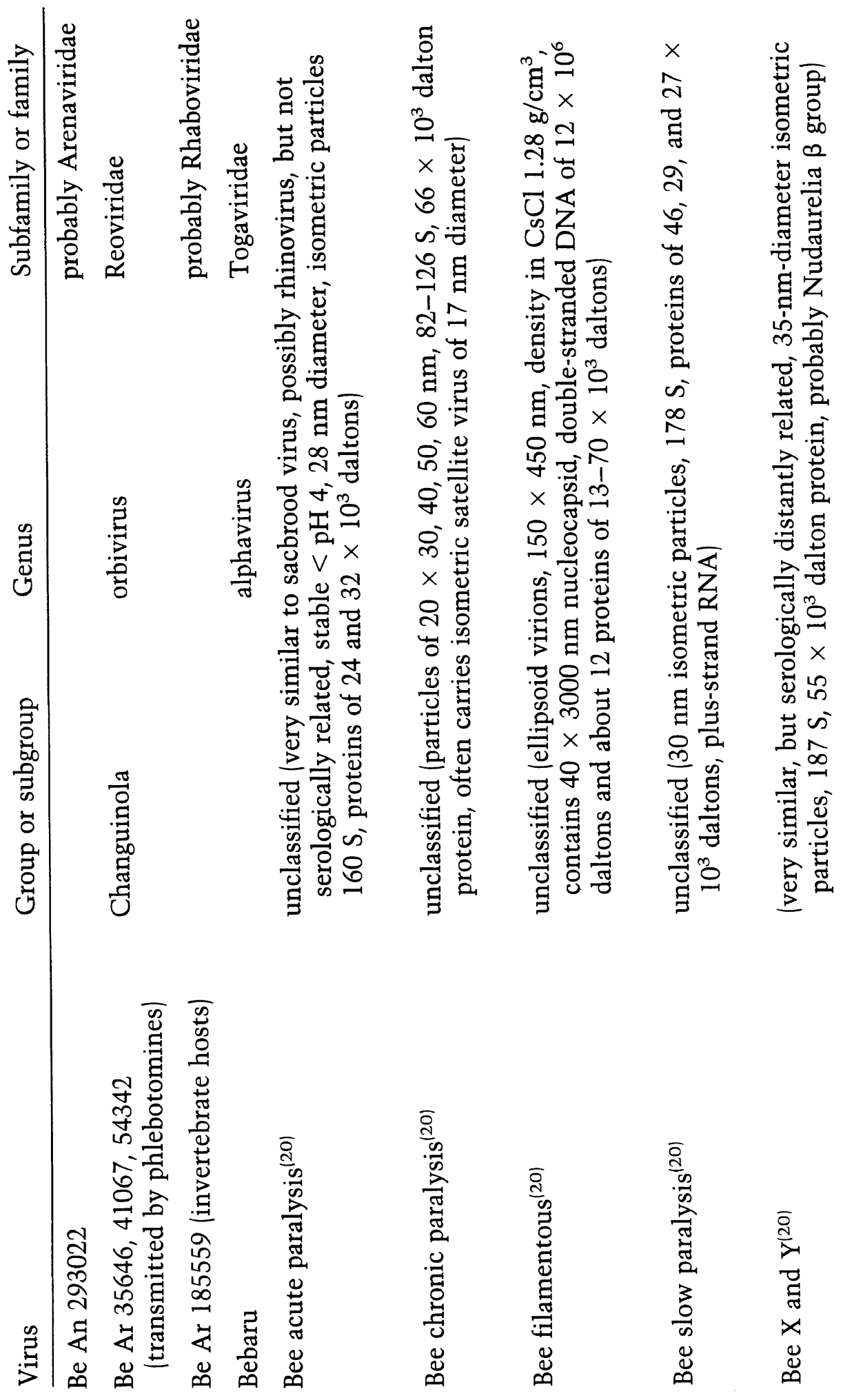


苞

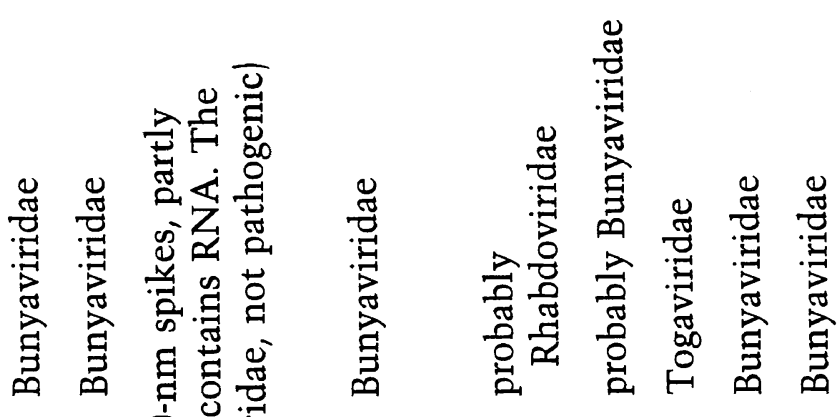

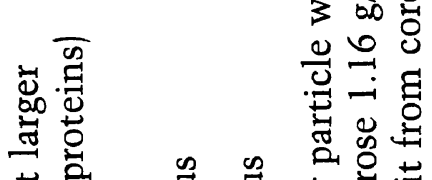

$\mathrm{O}_{n}-\mathrm{s}$

동 켱

Ф म $\mapsto$ ص

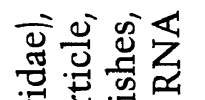

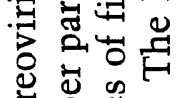

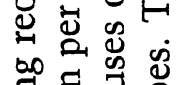

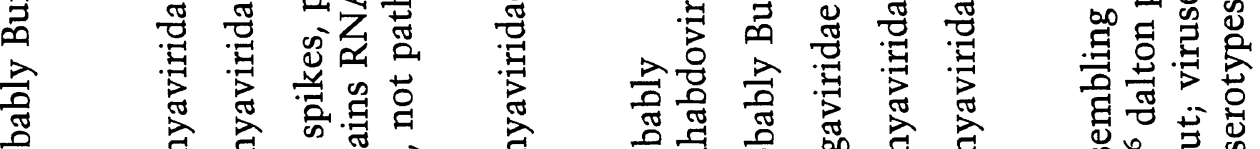

뭉

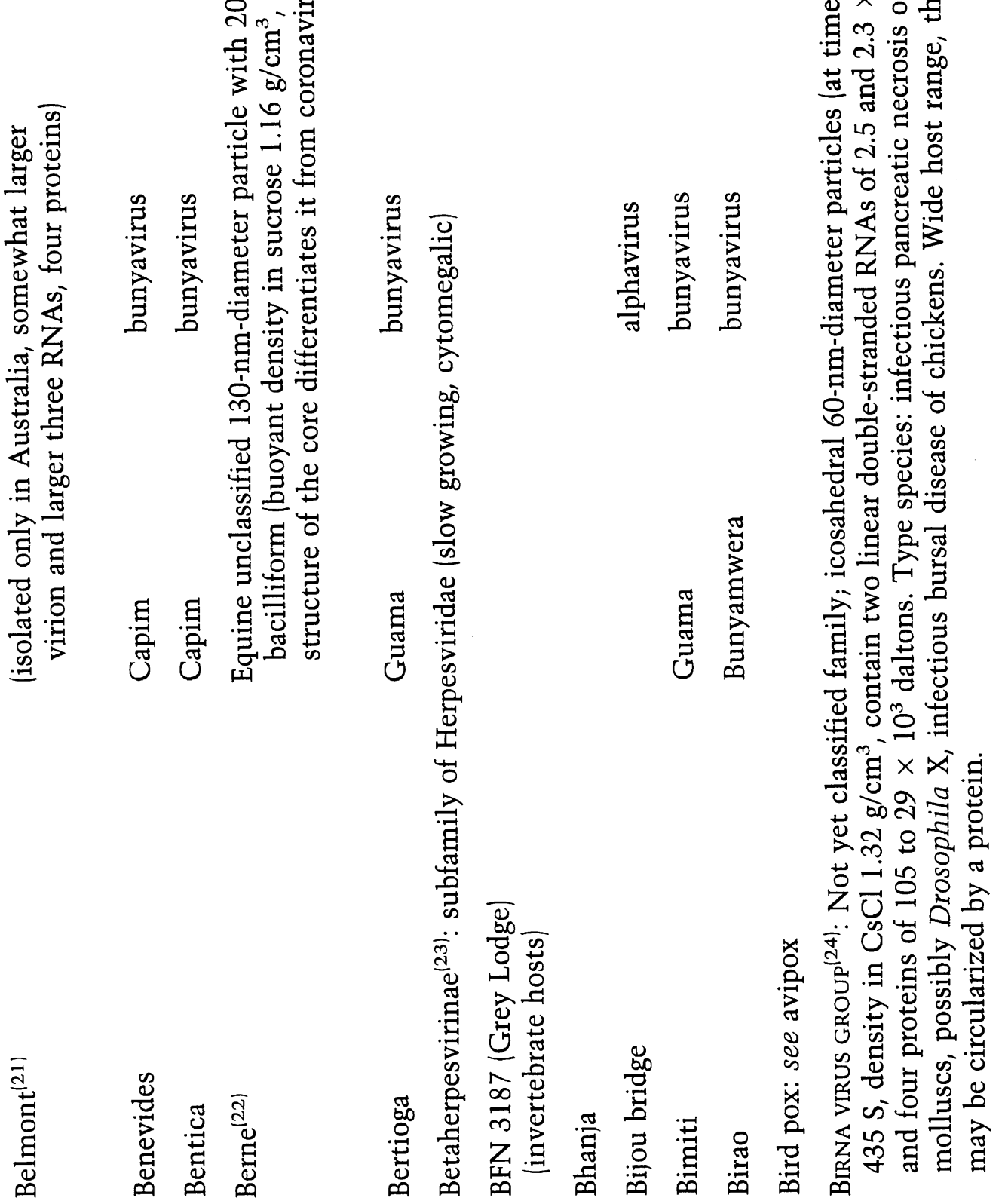




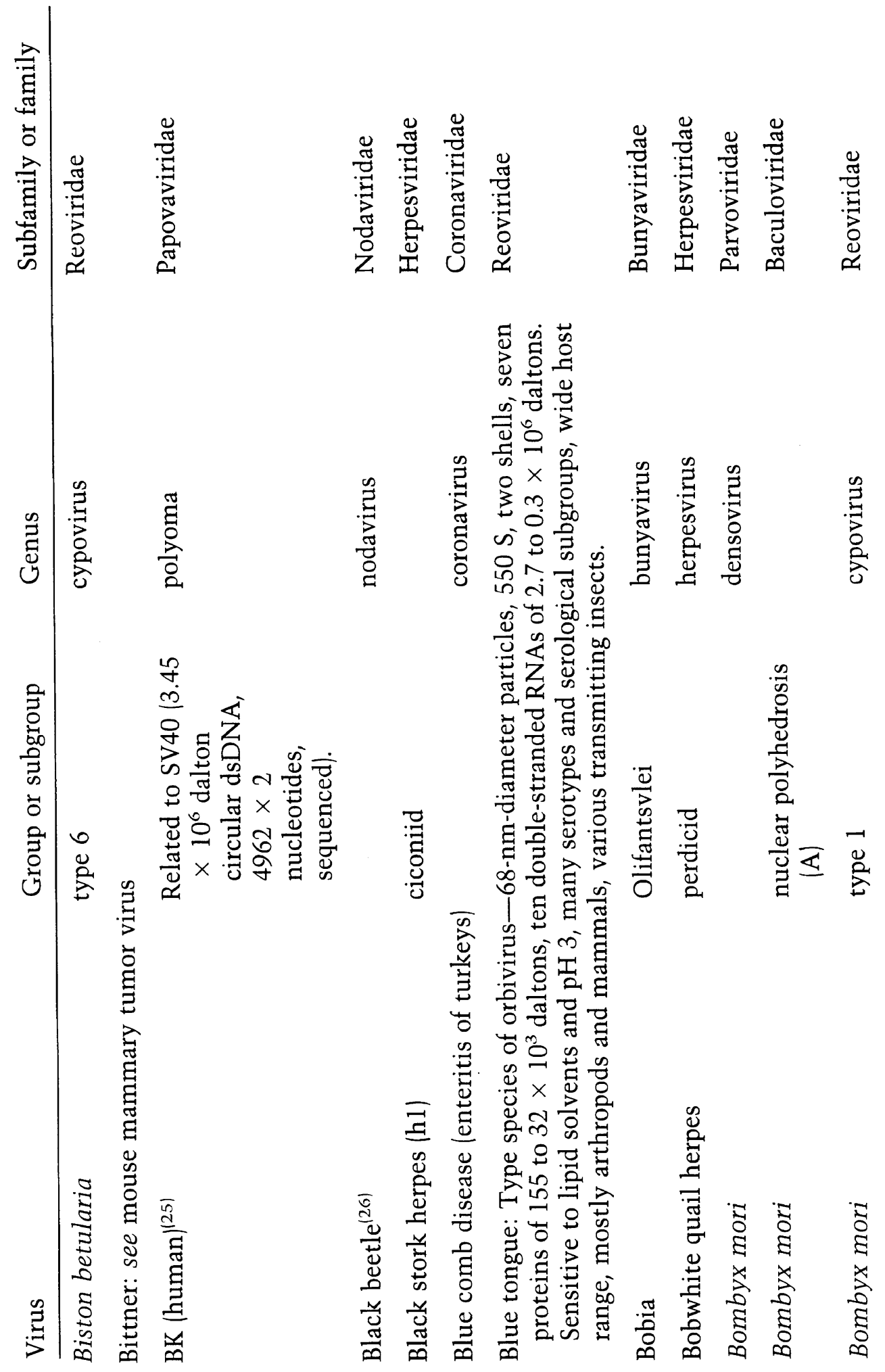



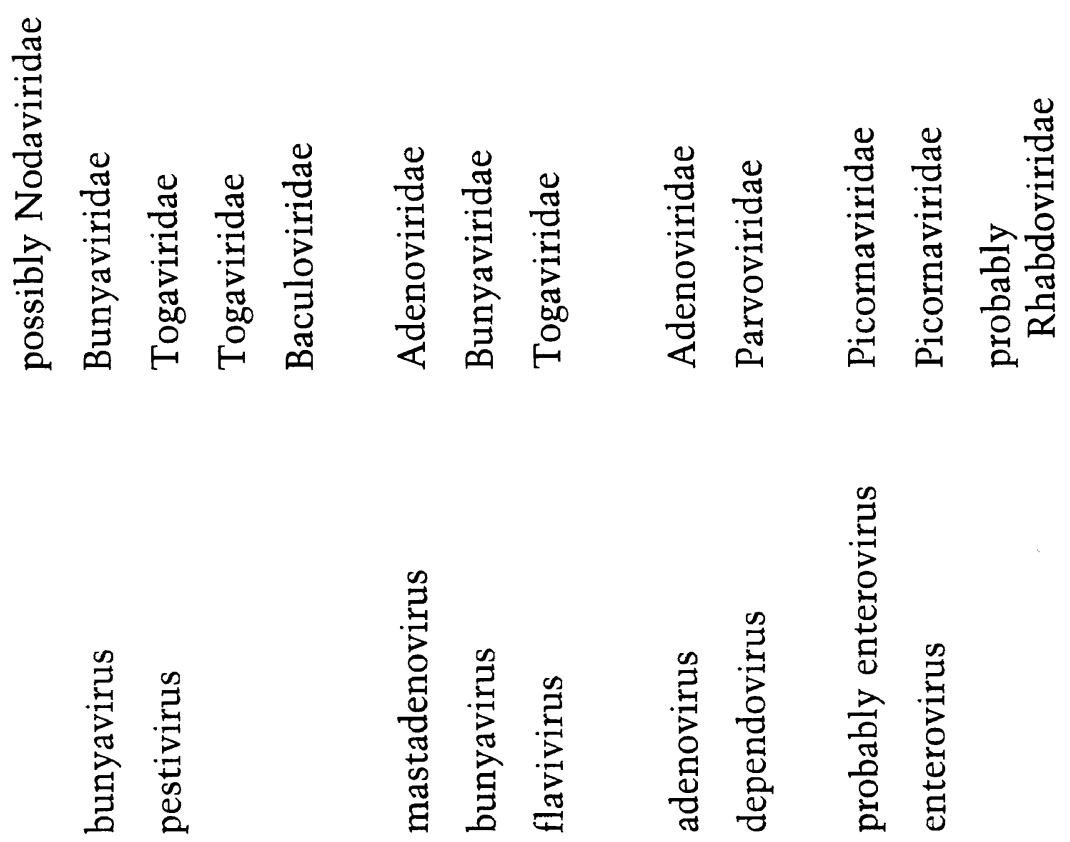

疍
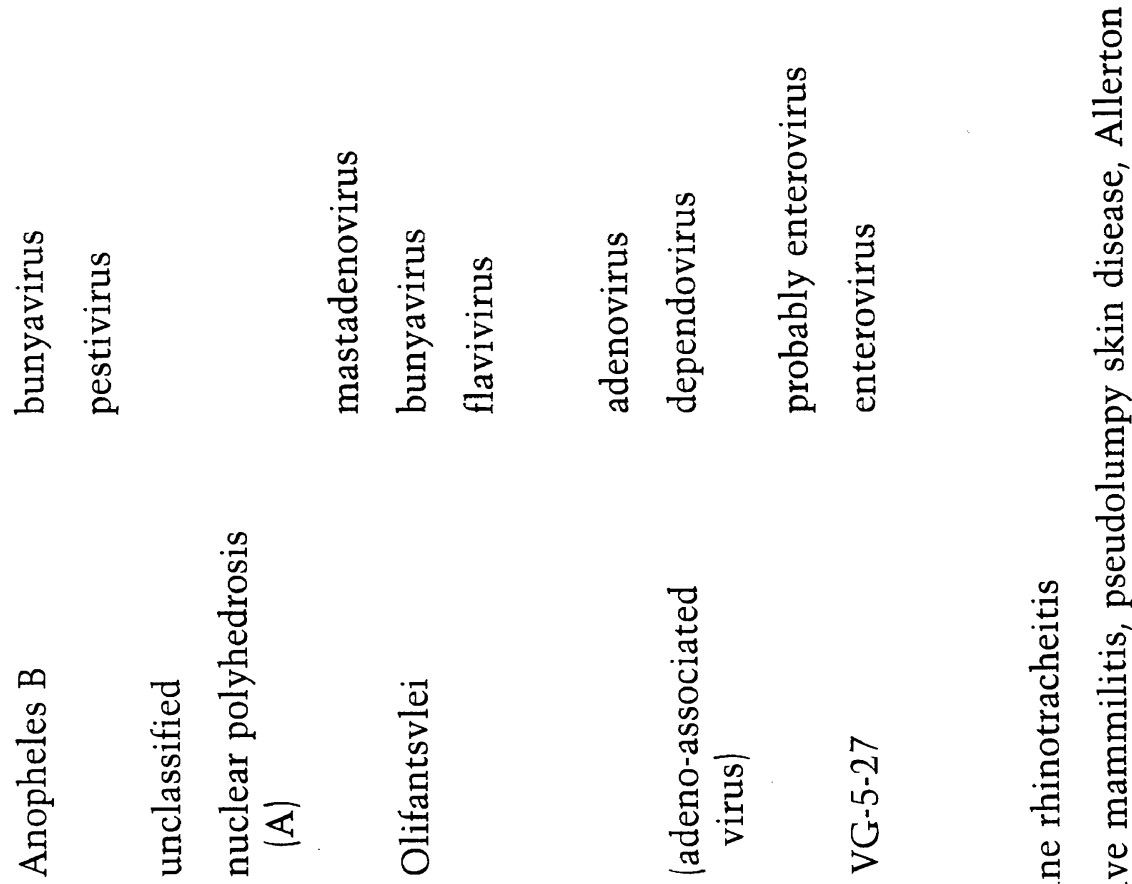

崩

$$
\text { 离 }
$$<smiles>C1COCCO1</smiles>

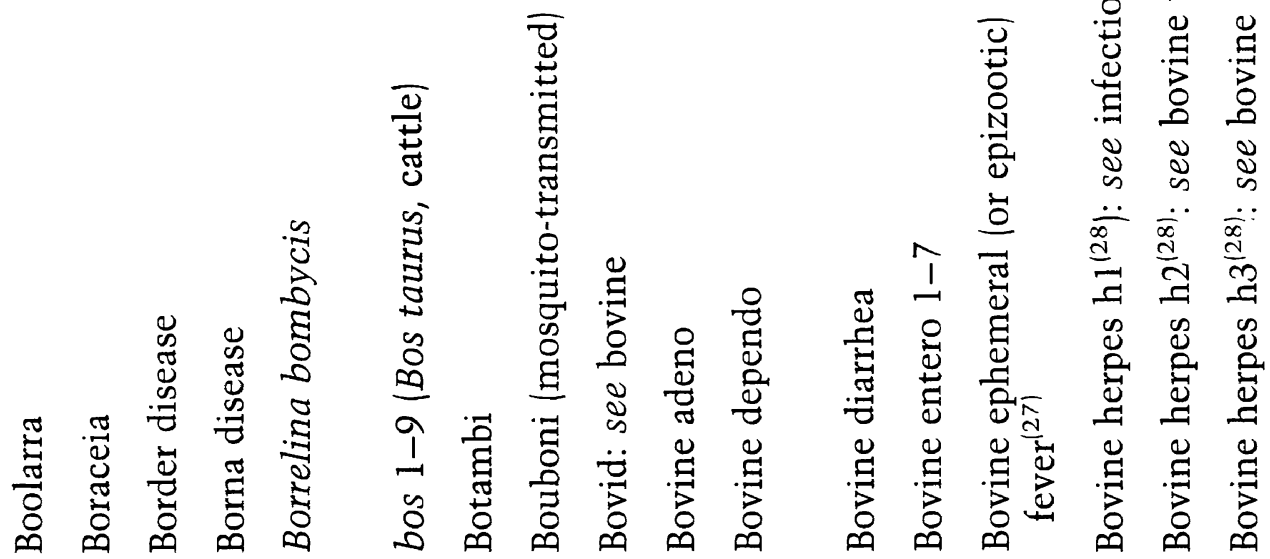



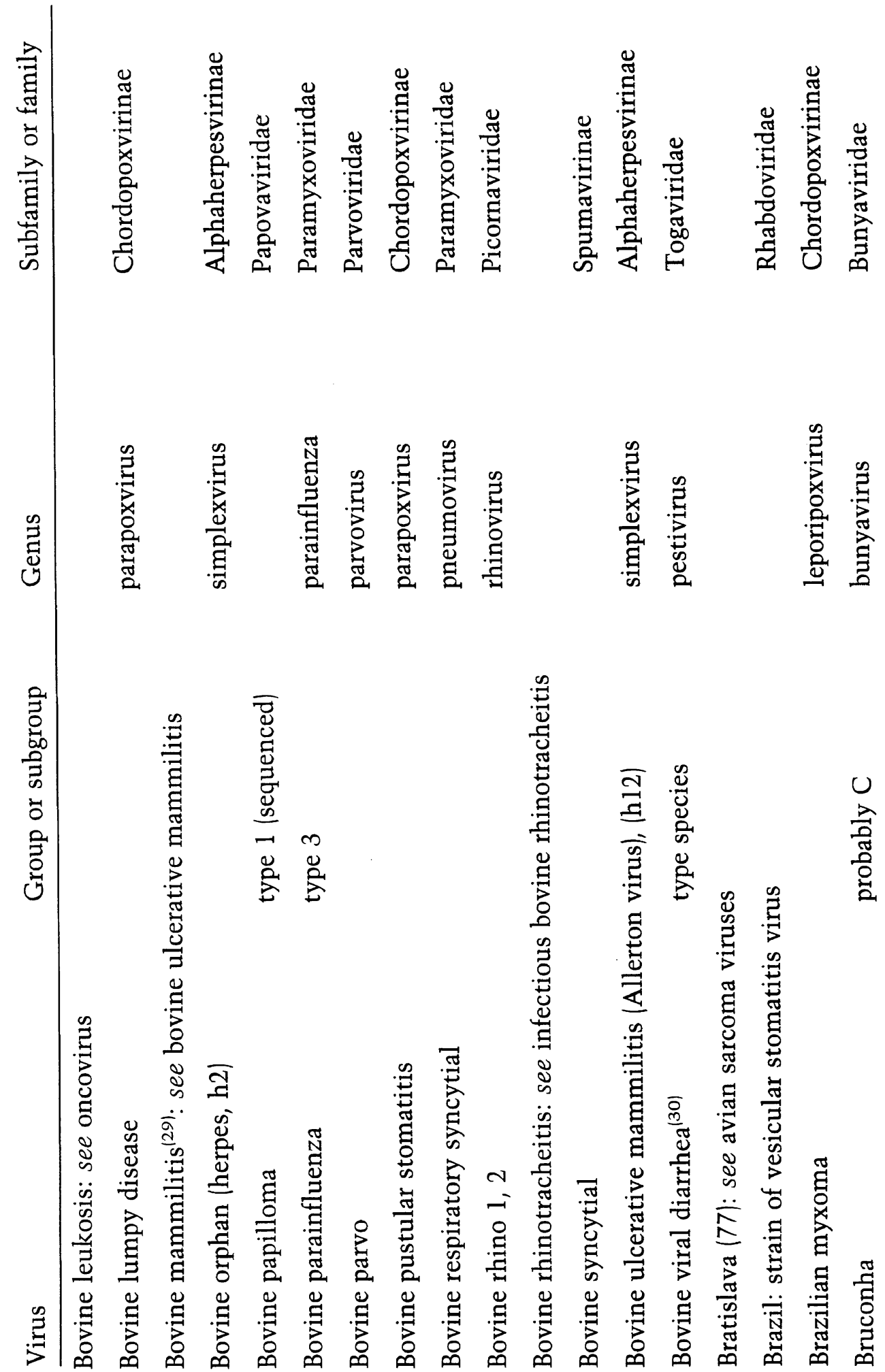


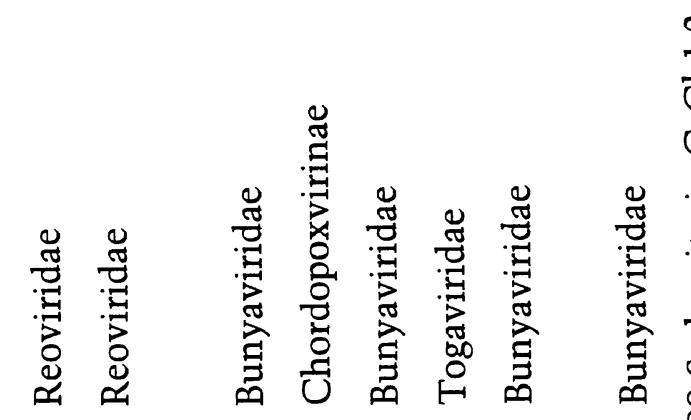

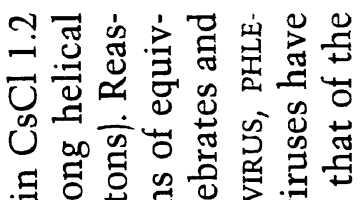

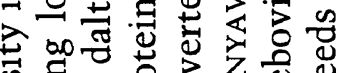

四

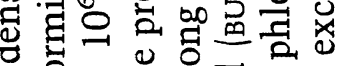

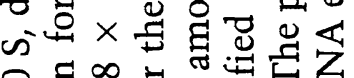

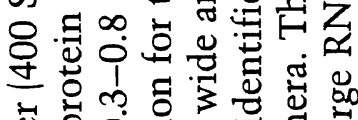

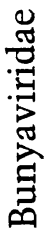

屯ี

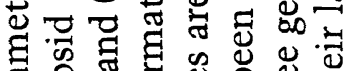

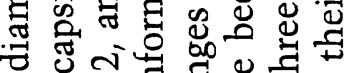

๑

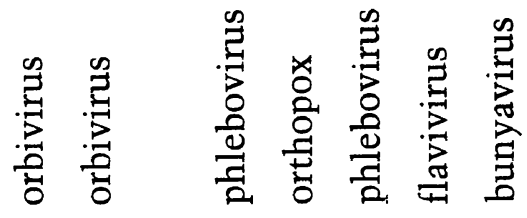

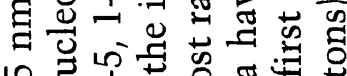

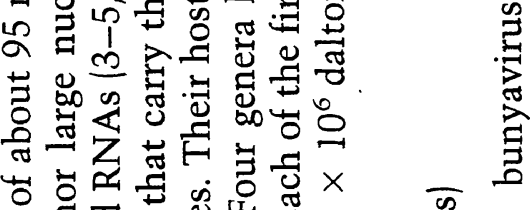

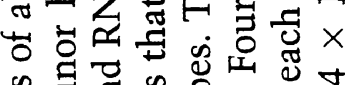

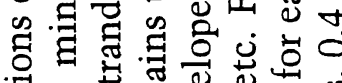

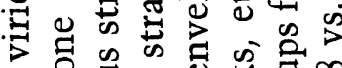

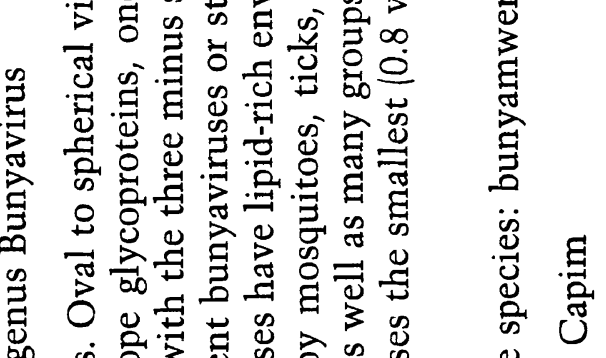

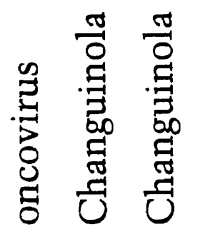

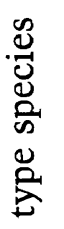

ญి

क म

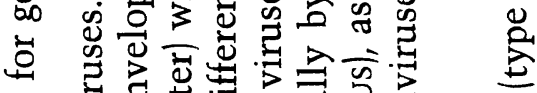

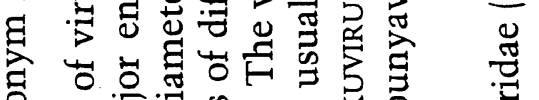

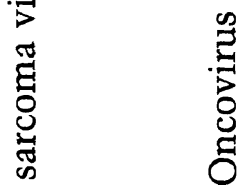

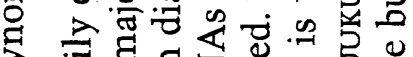

क द्व

苛 恶 放

పू०

$\begin{array}{ll}0 & 8 \\ 0 & \infty \\ 0 & \ddot{1}\end{array}$

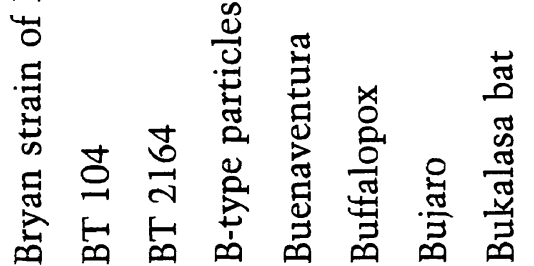

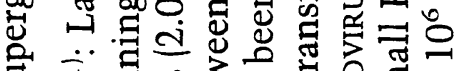

क

苞

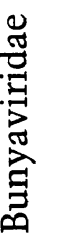
. 


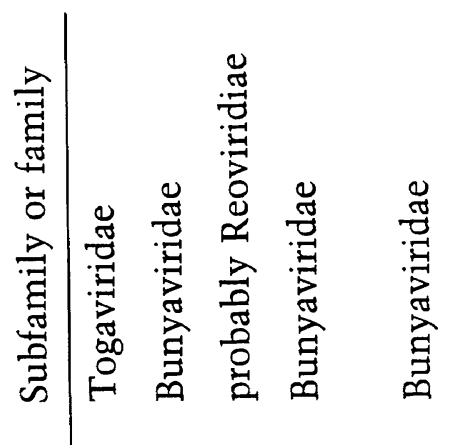

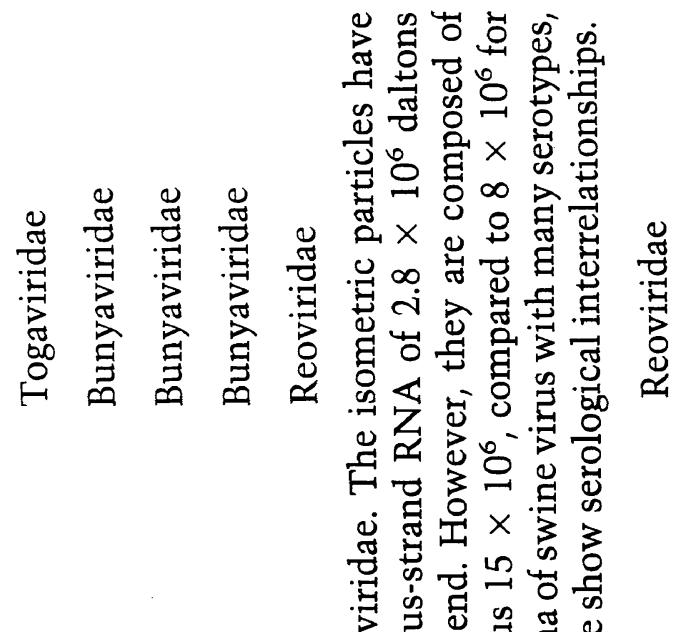

至

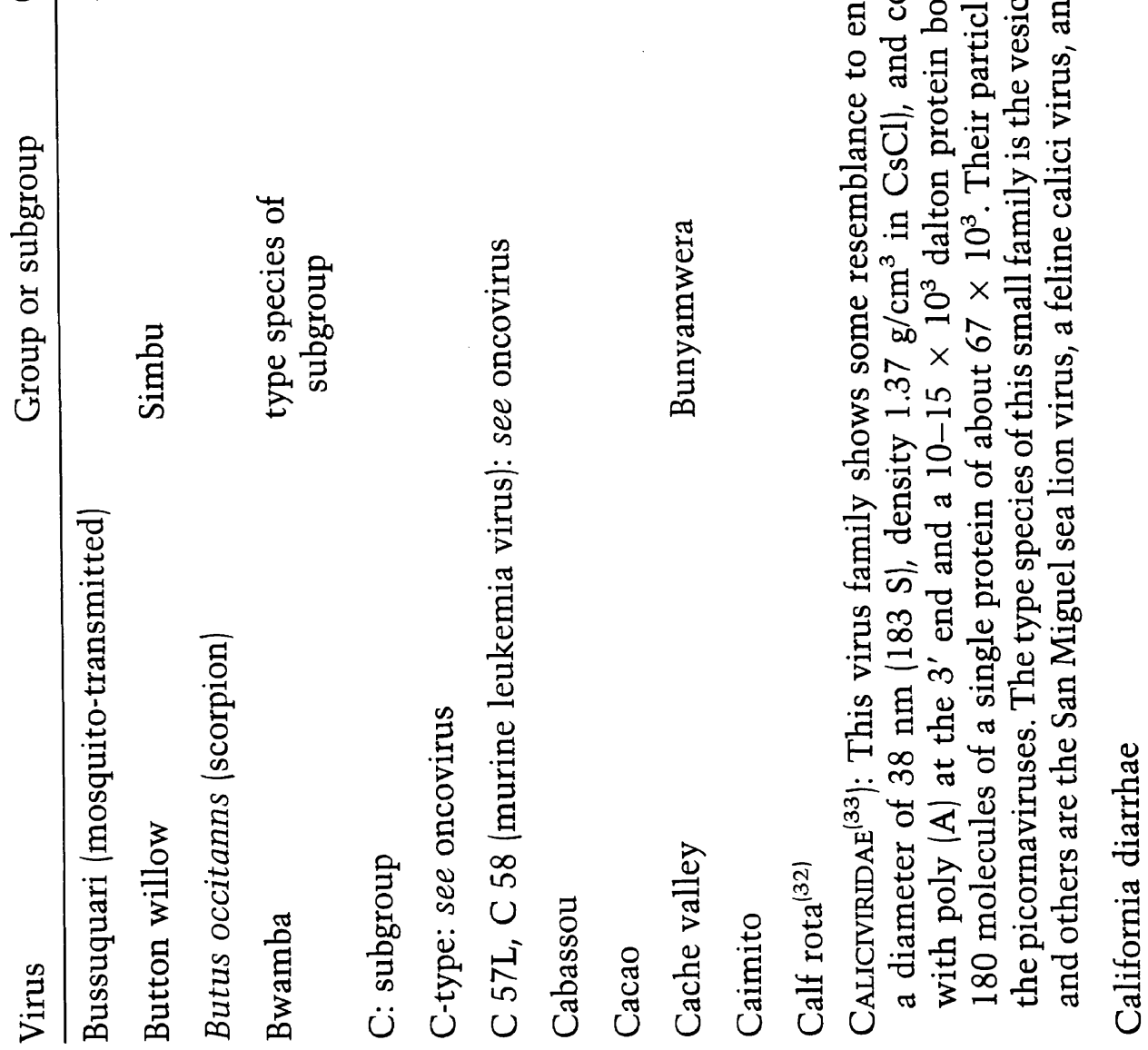



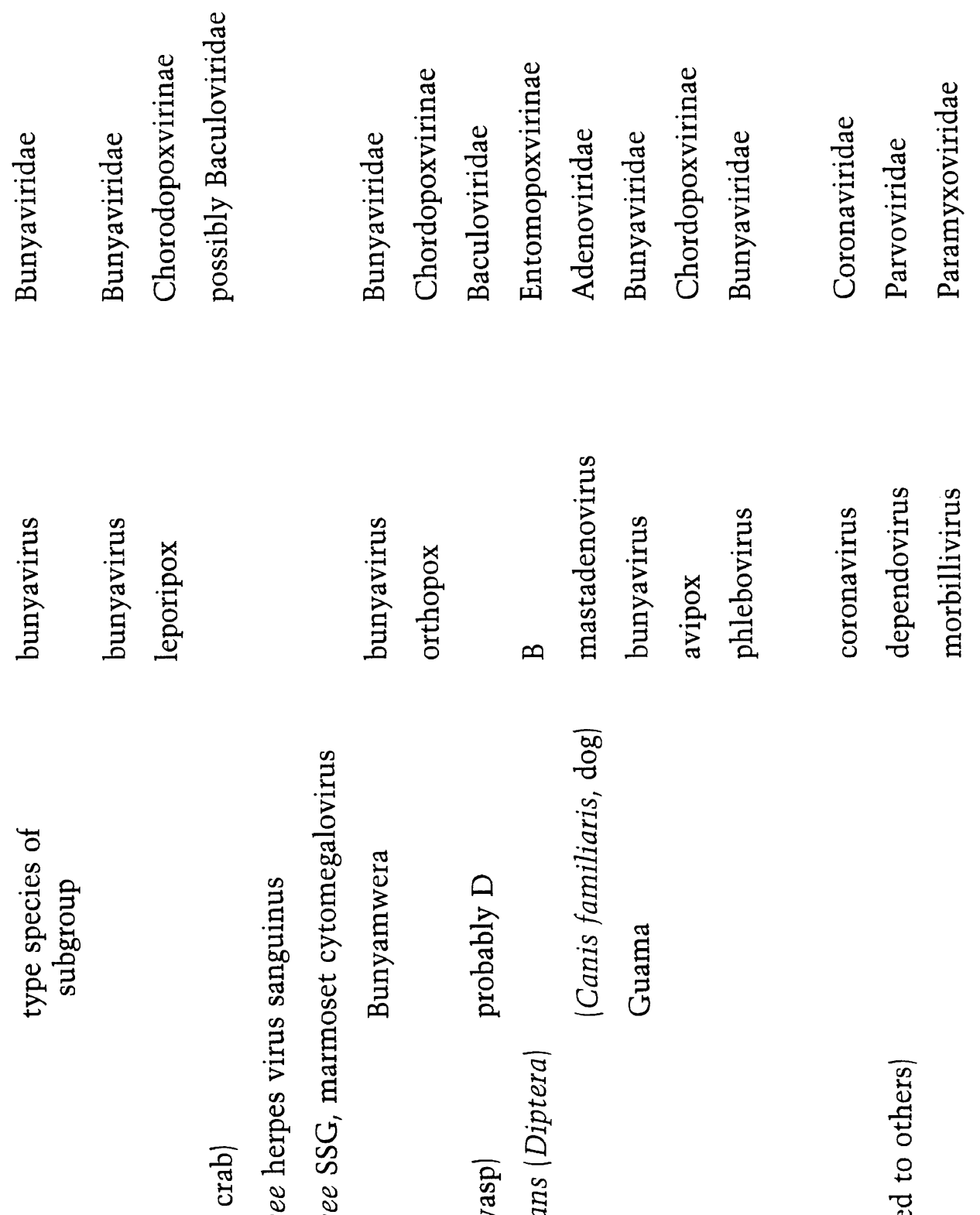

离
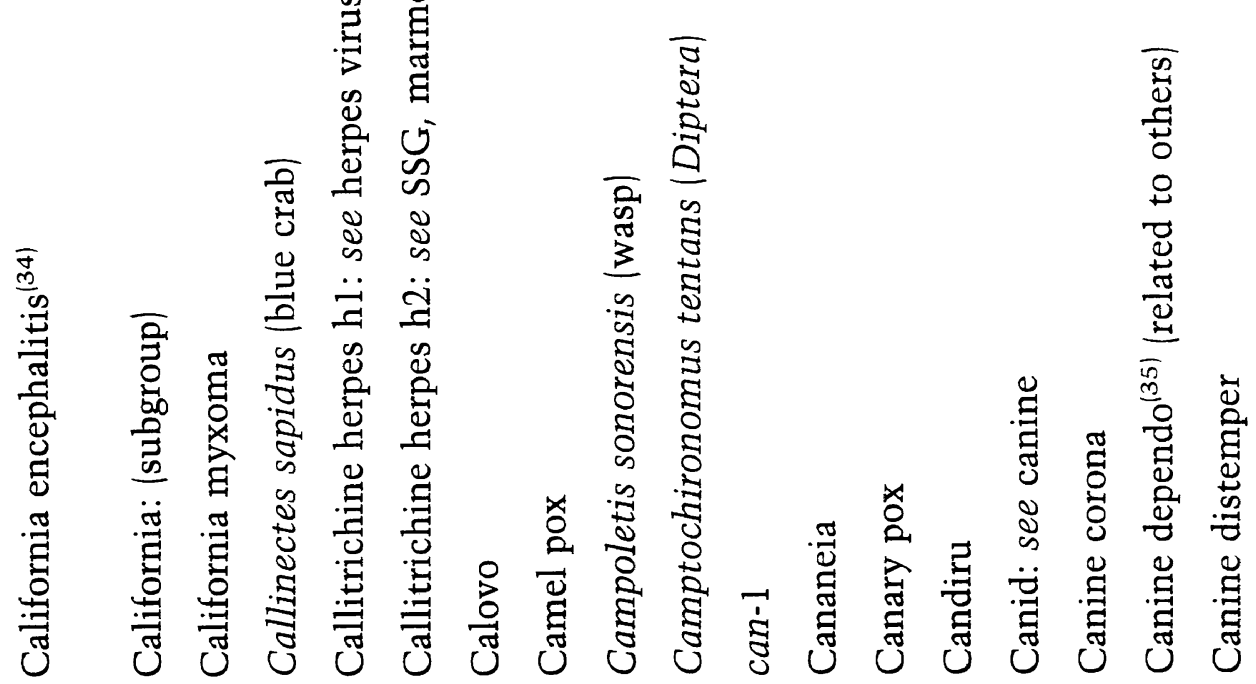

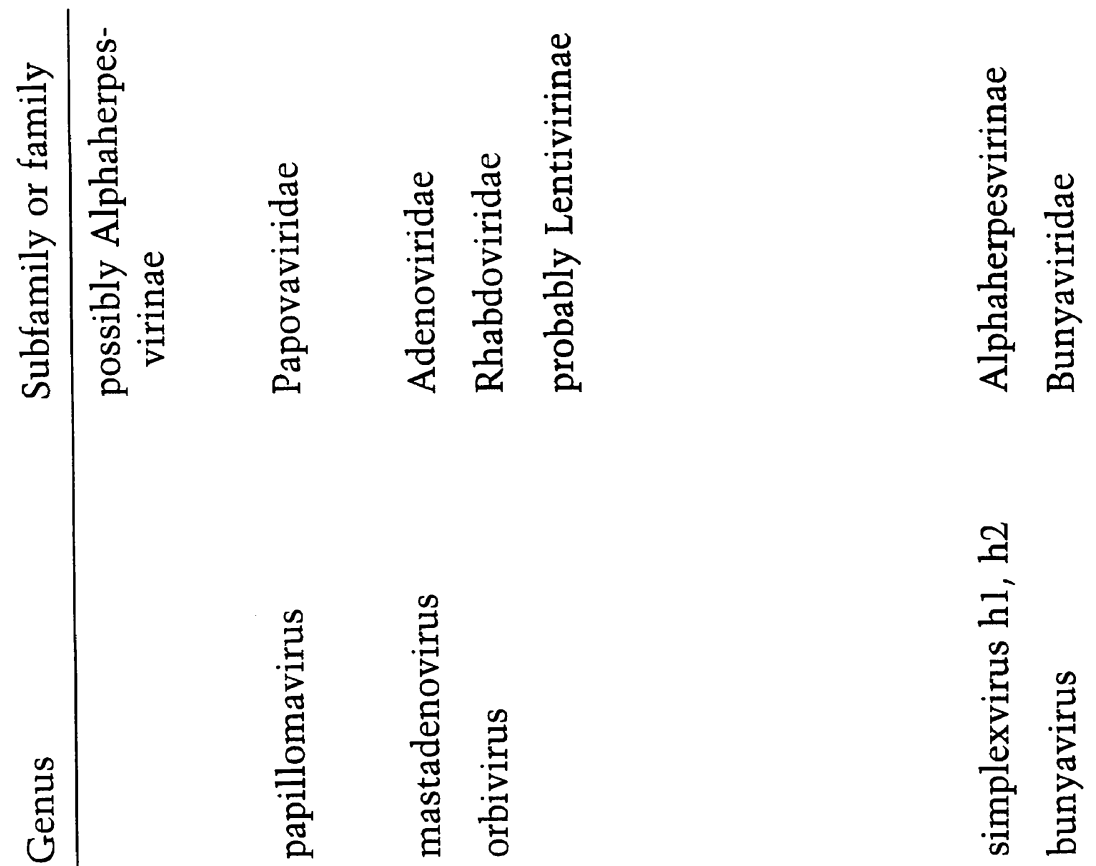

조

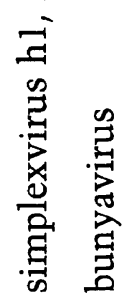

忽<smiles>C=CC#CC=CC=C</smiles>
范

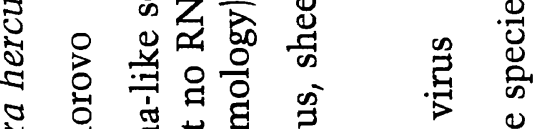

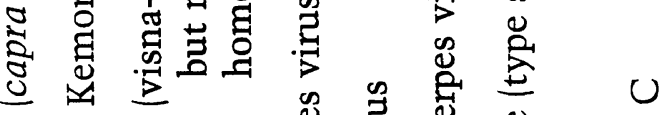

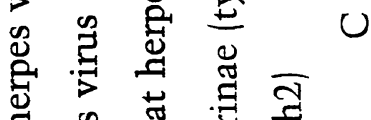

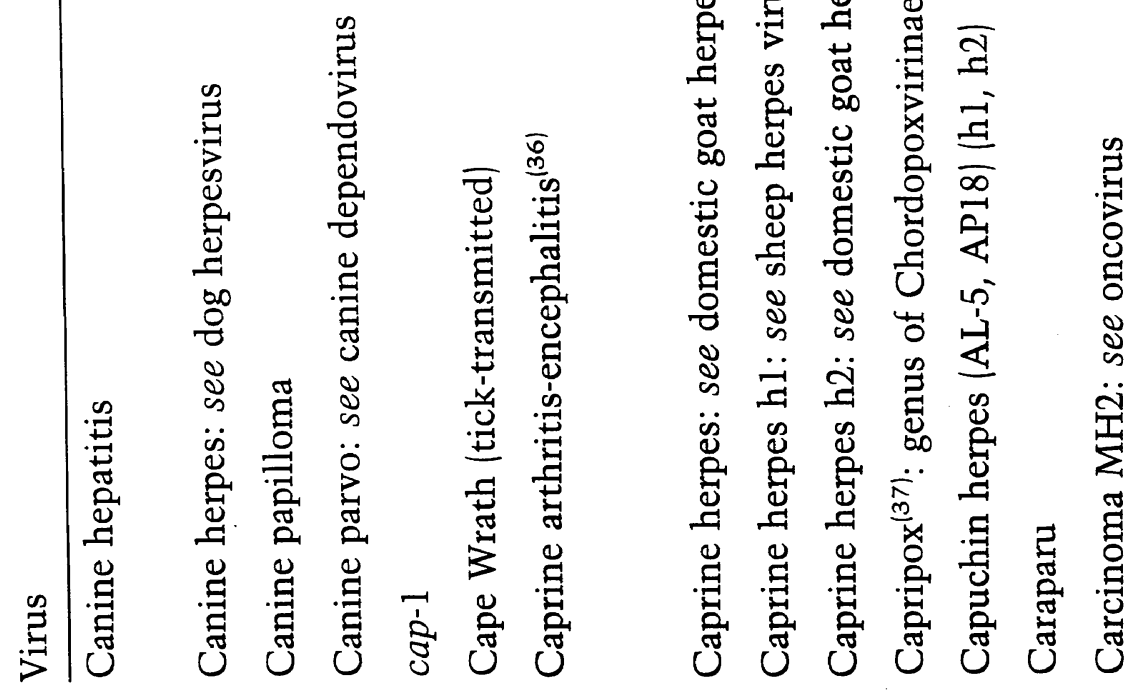



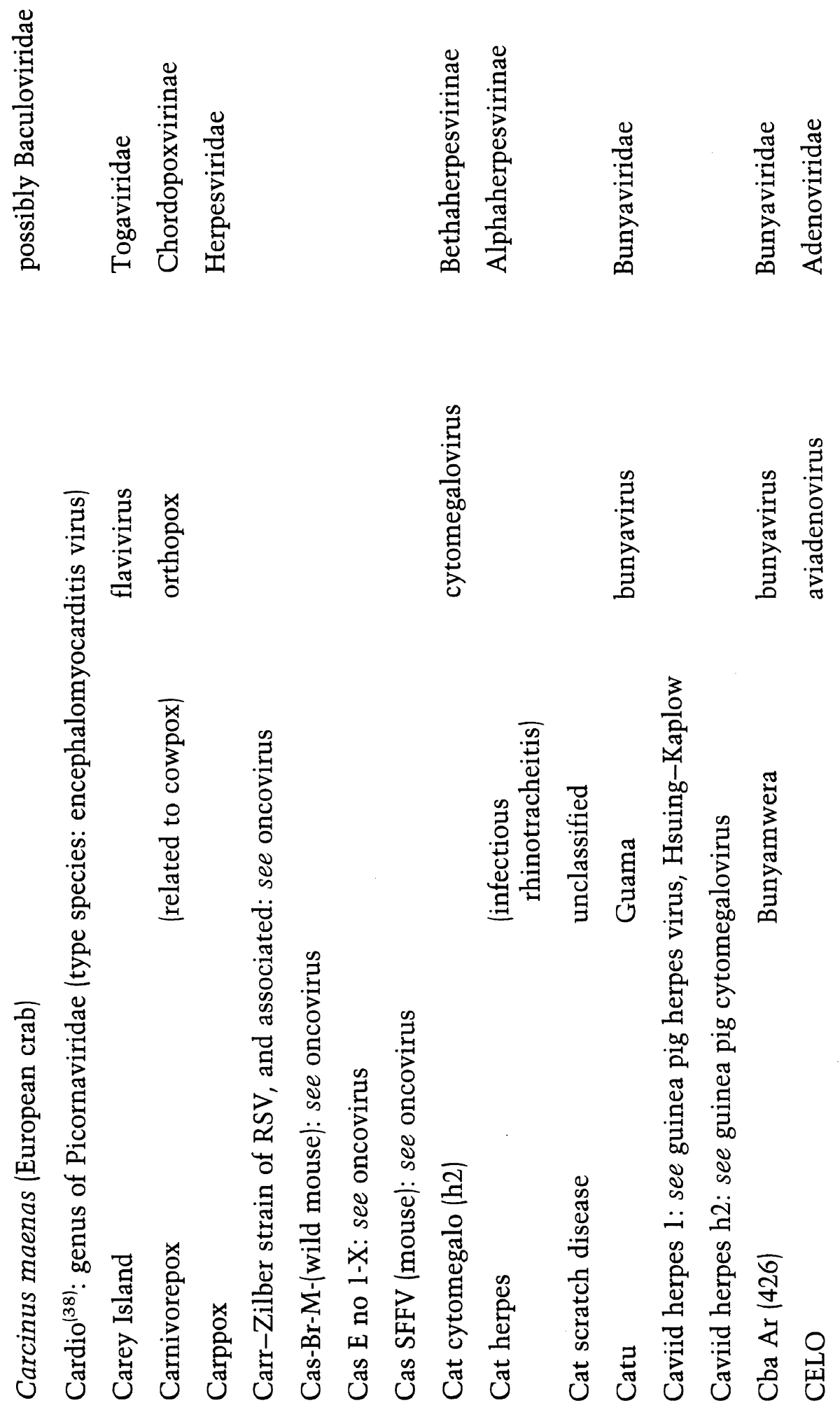


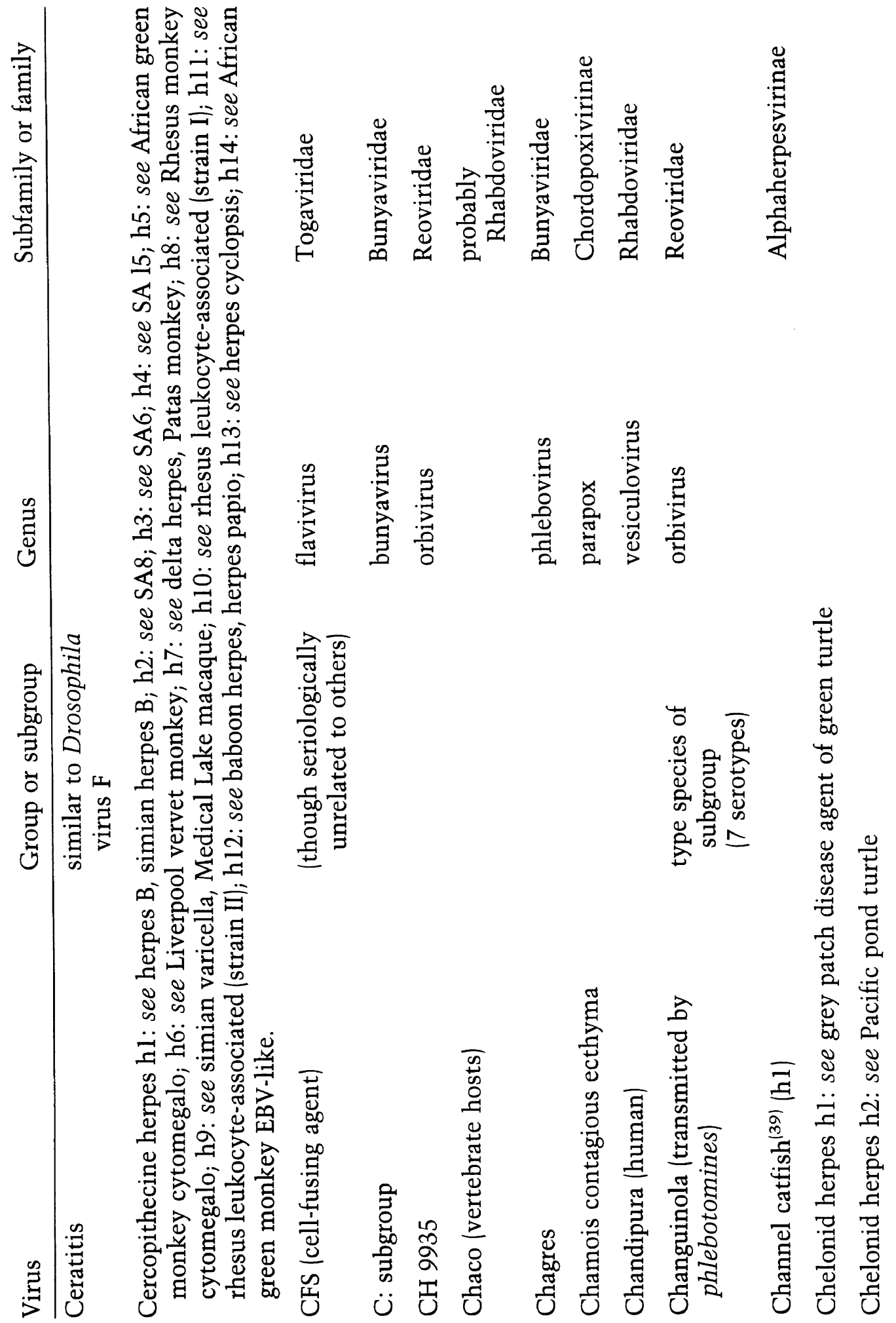



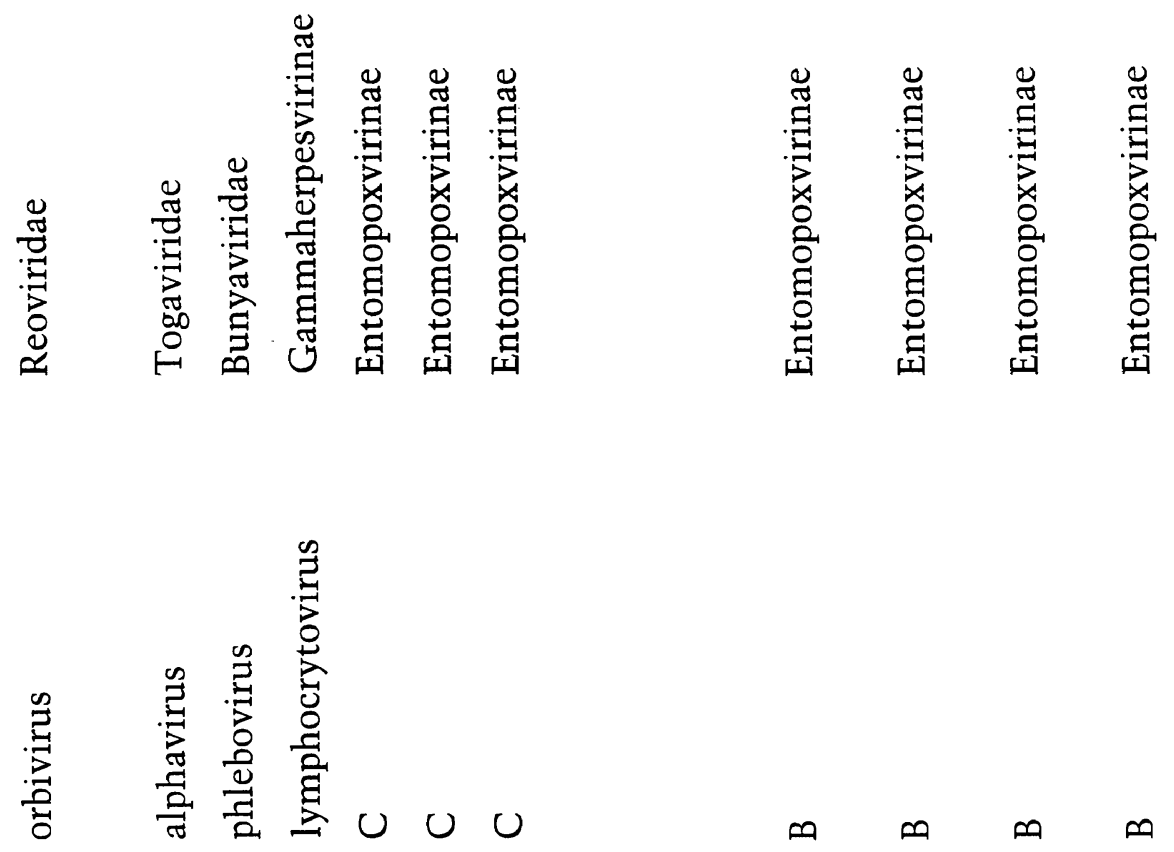

$\infty \infty \infty$

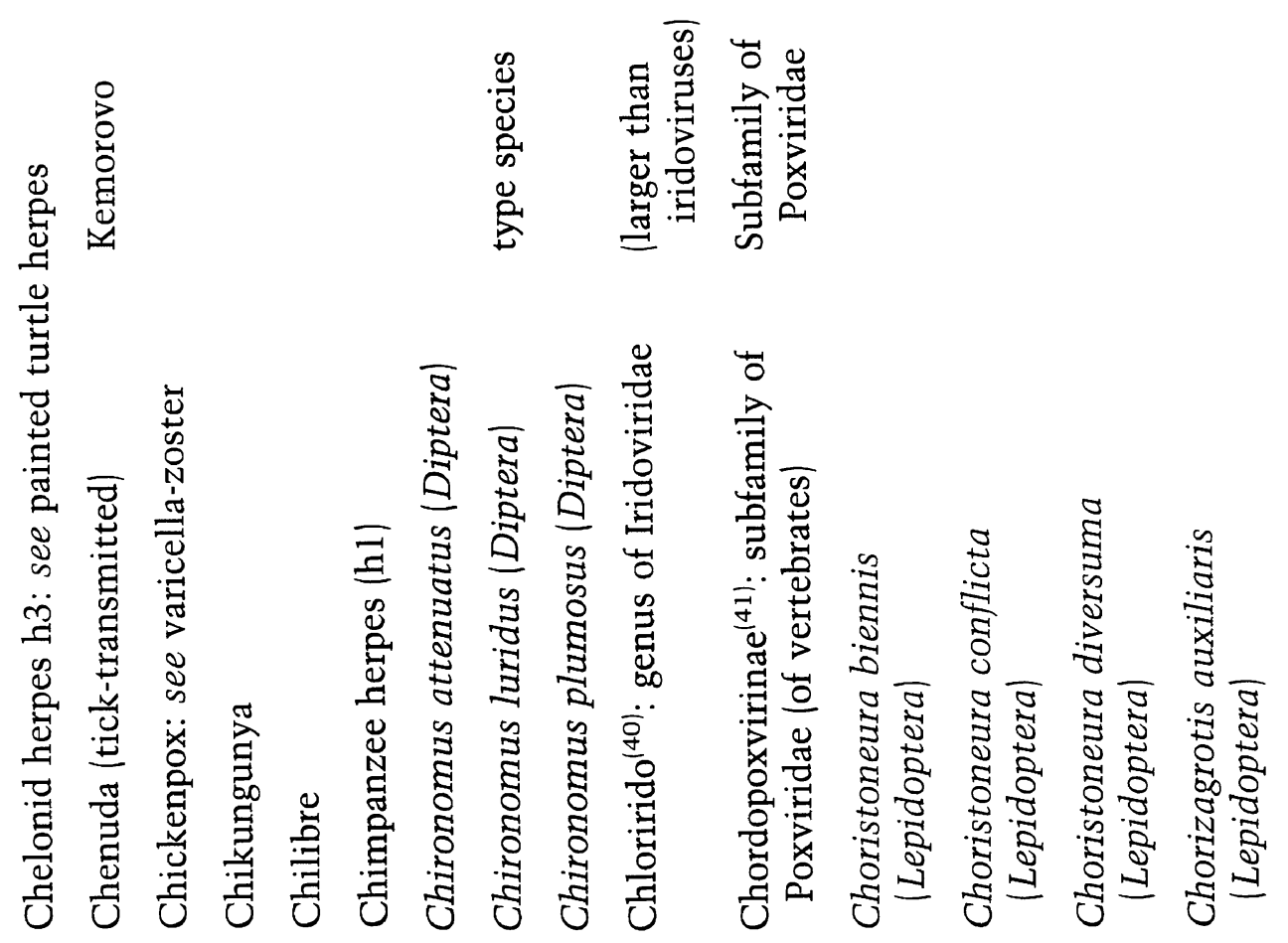




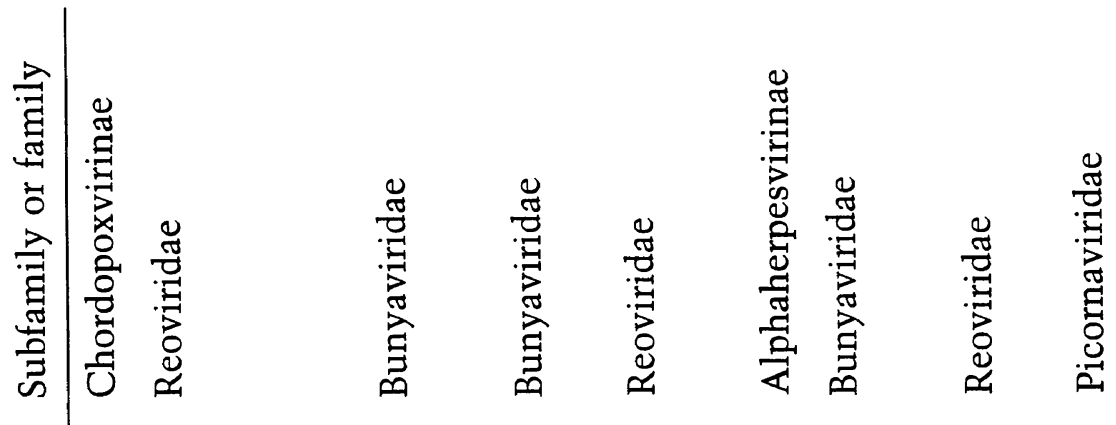

壱

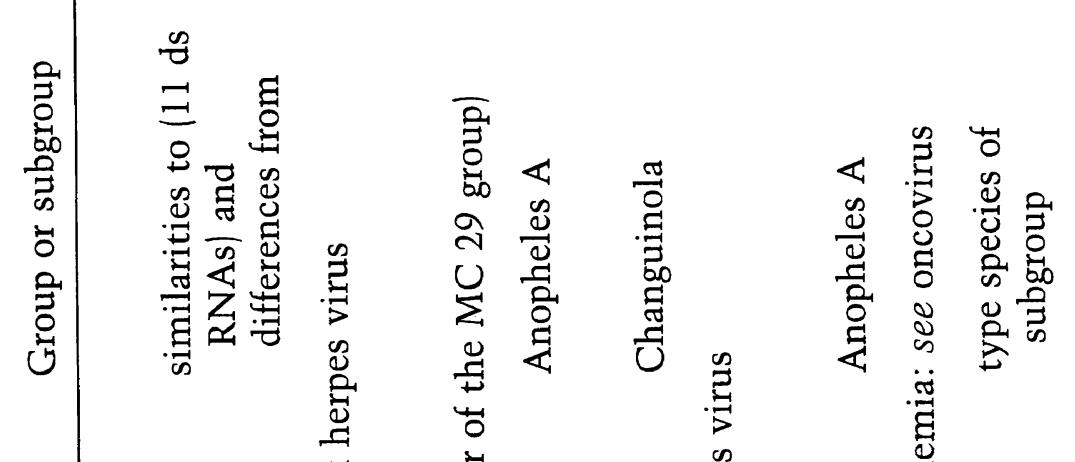

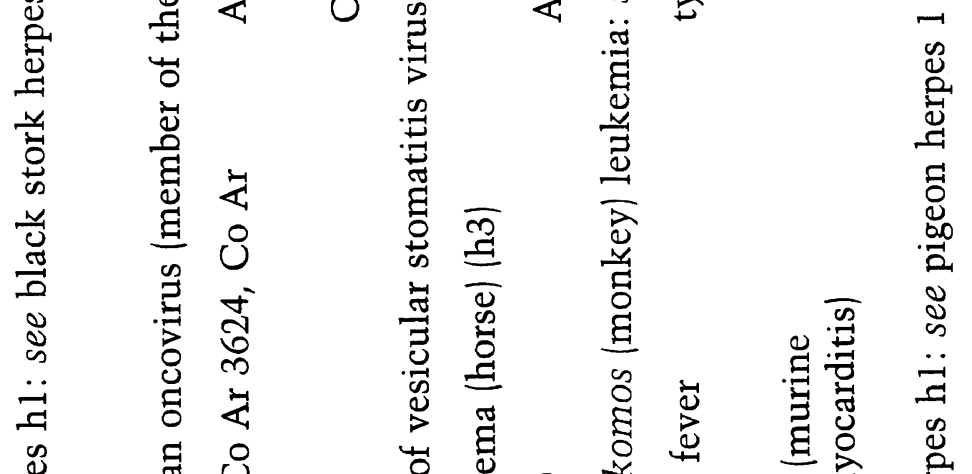

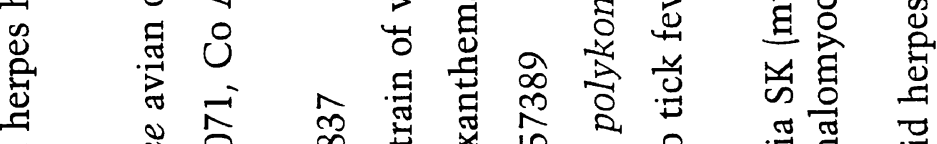

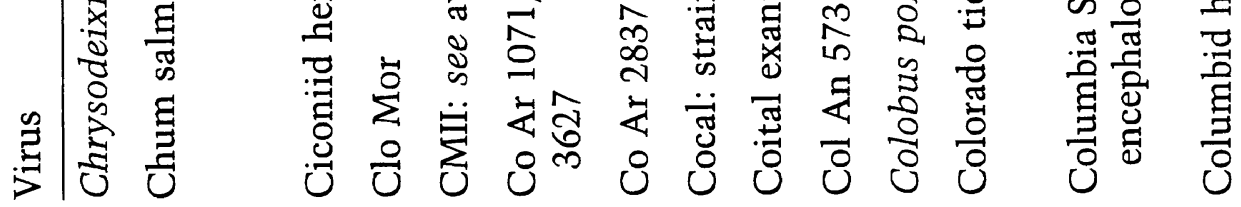




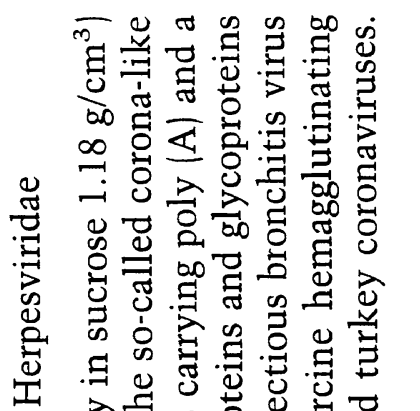

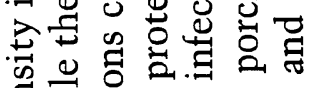

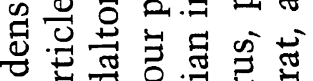

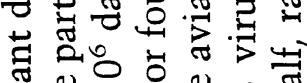
สิษ 䒠可式䓌 อ : 20 F

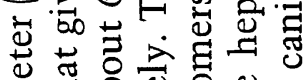

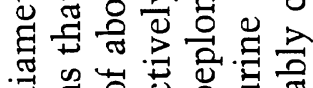

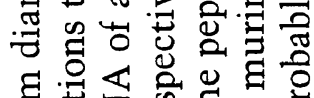

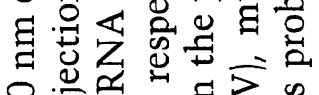

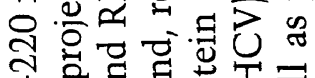
กิ

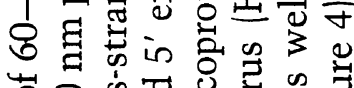

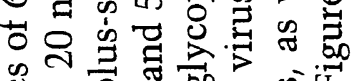

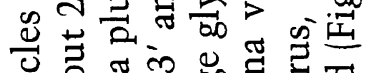

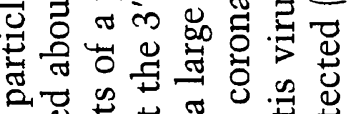

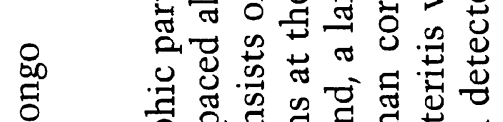

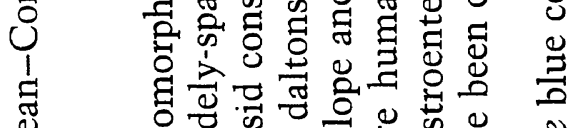

$\stackrel{\dot{3}}{\supset}$
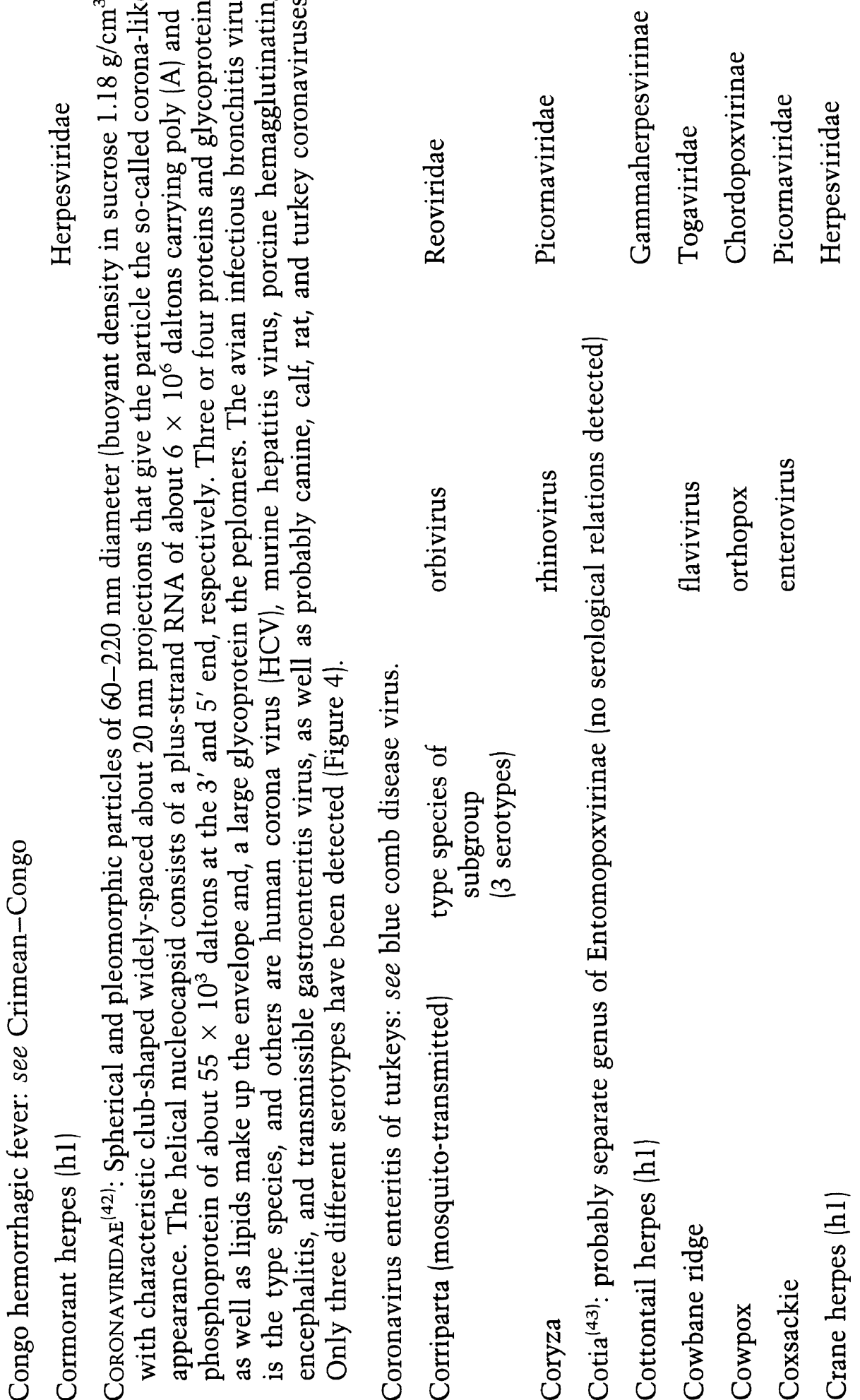

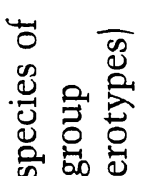

苛

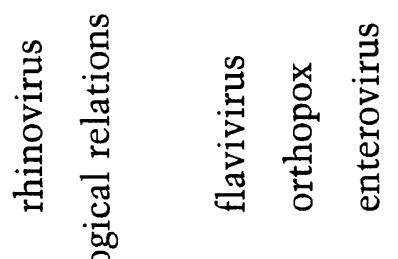

只舟

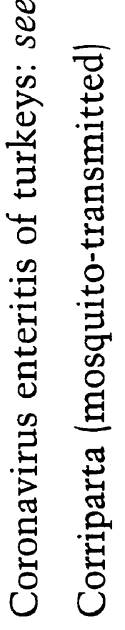

융

을

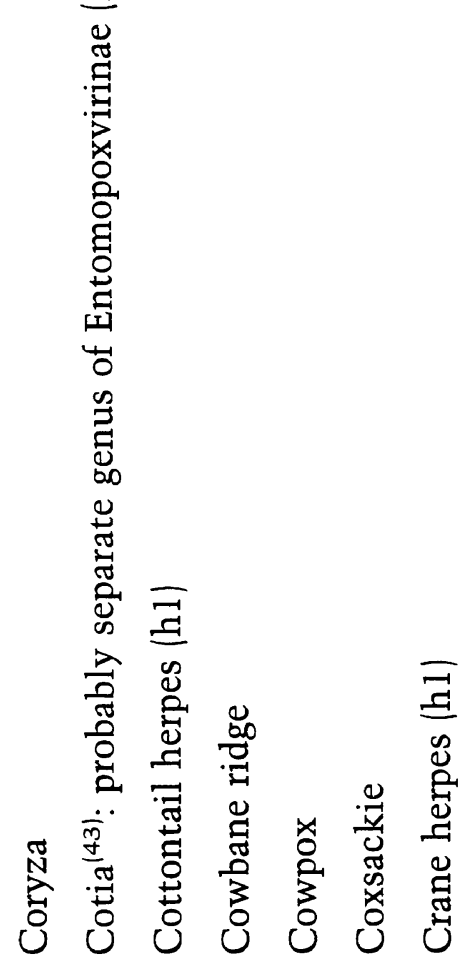




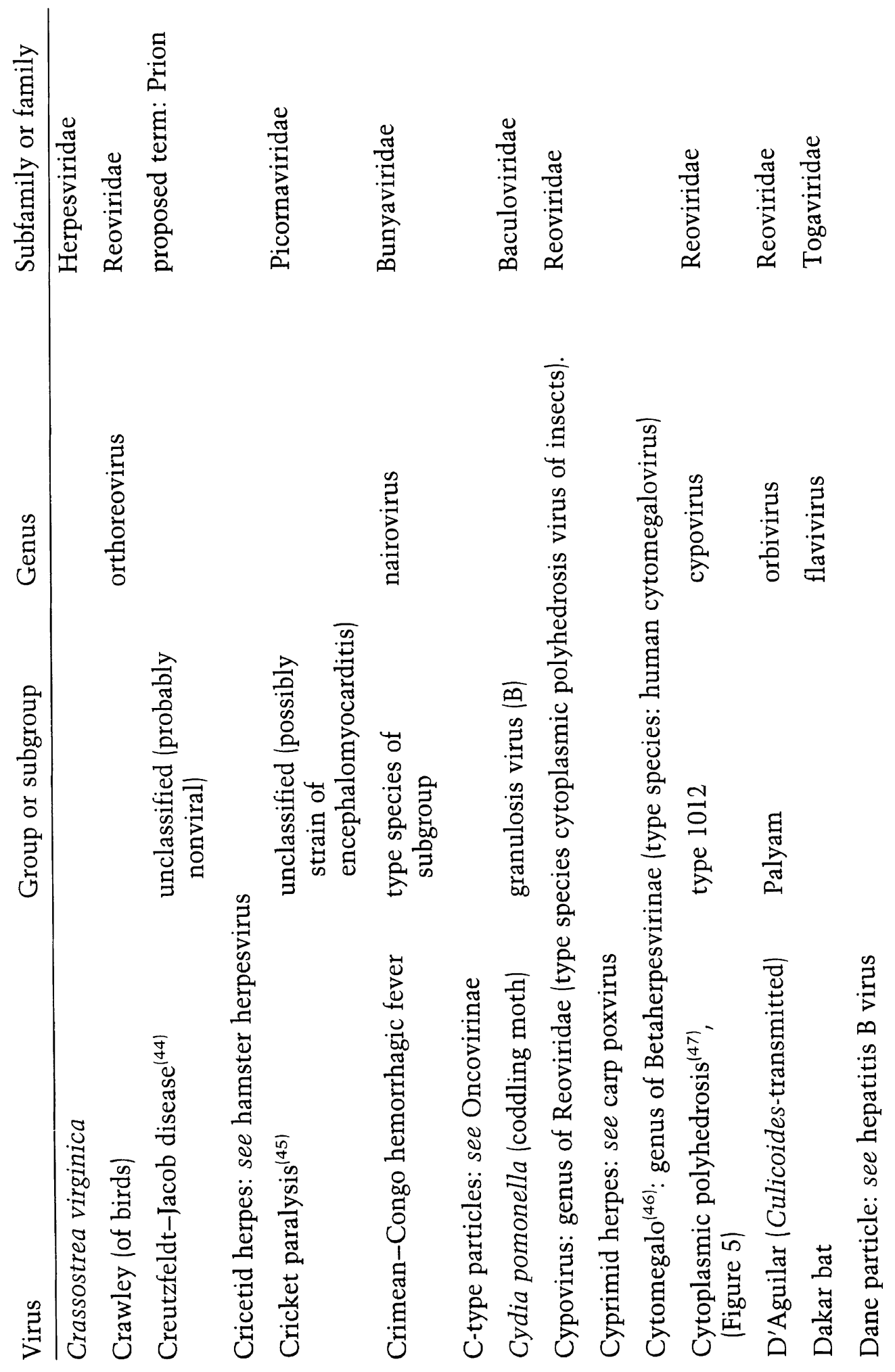




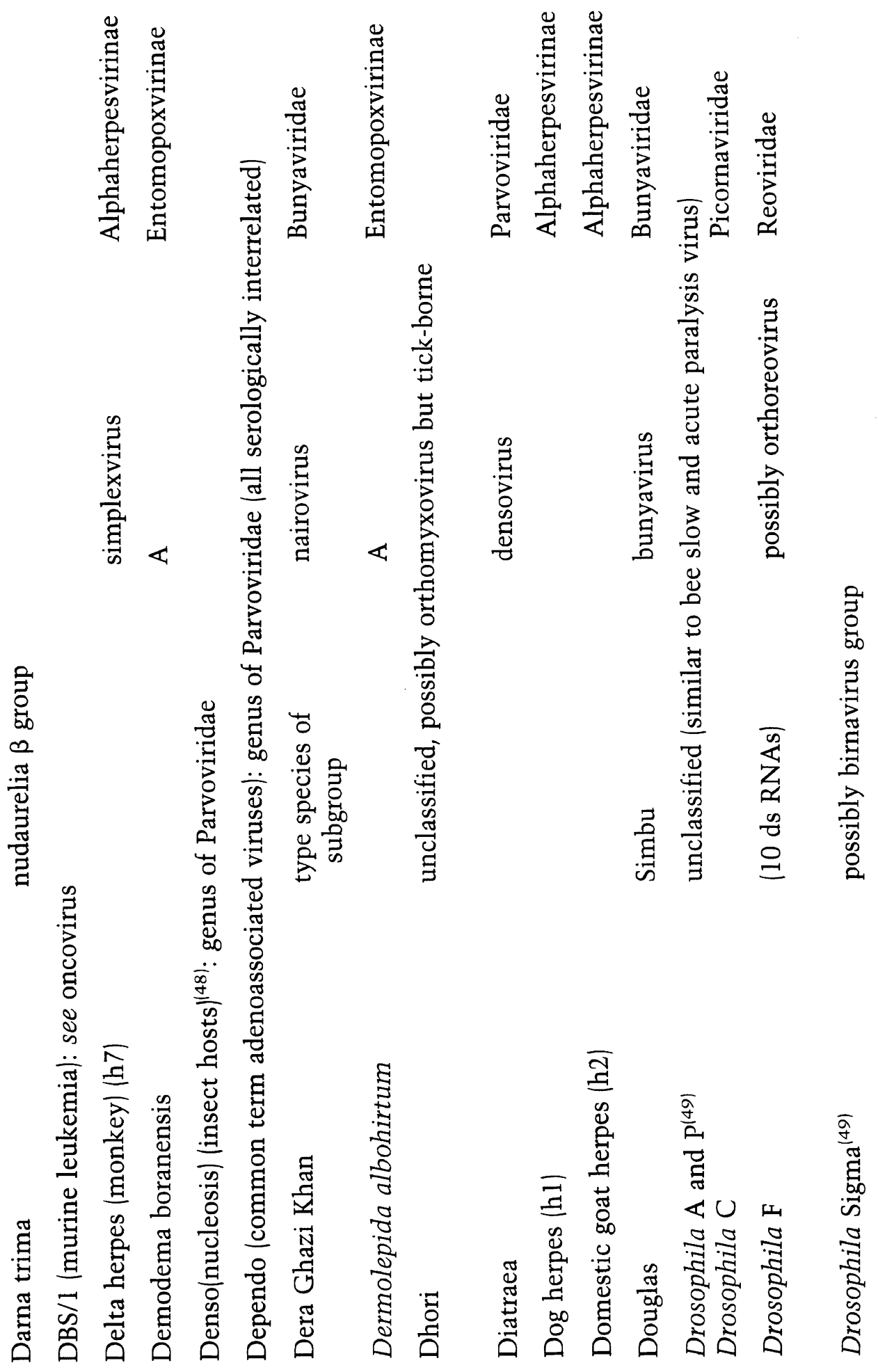



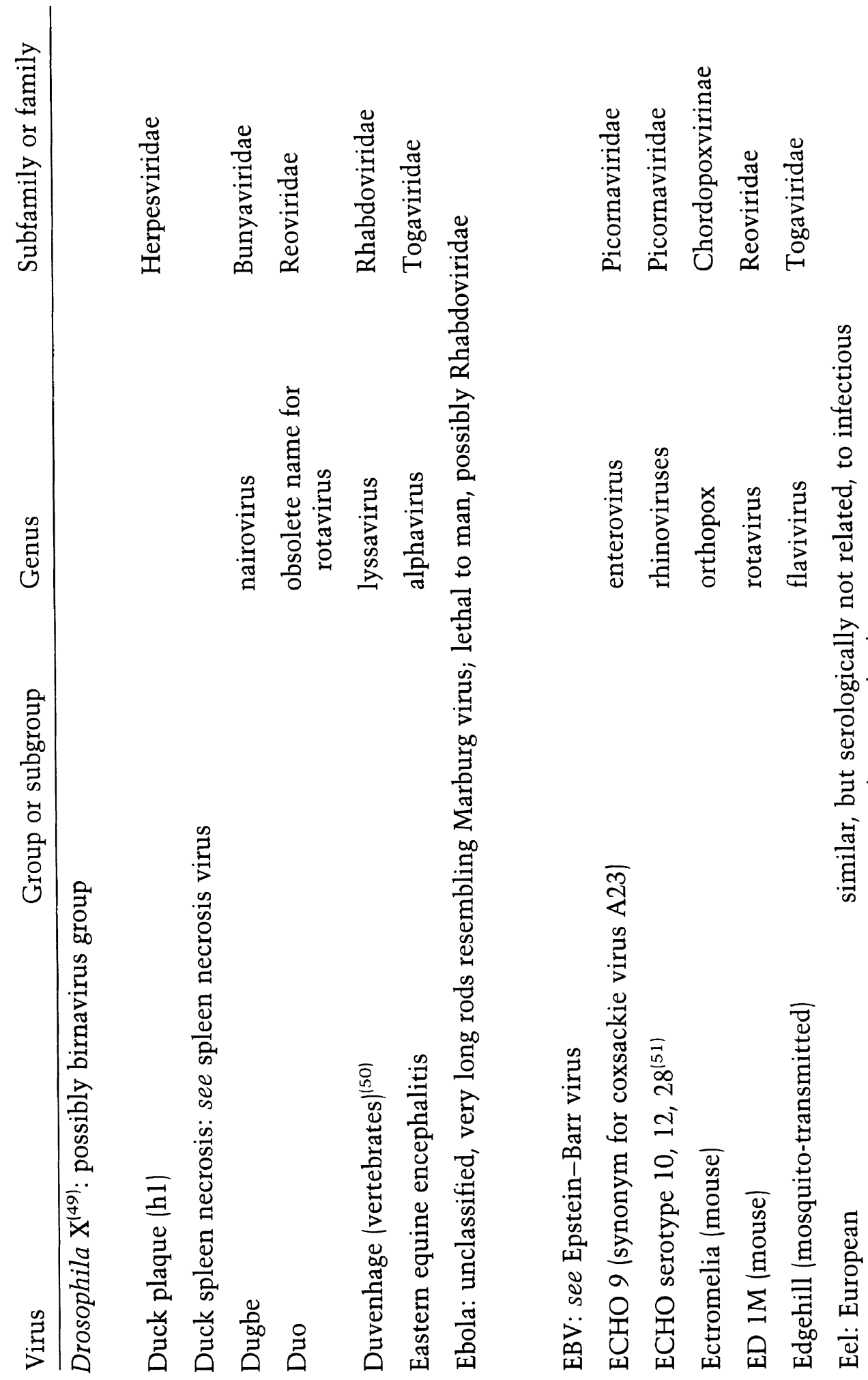

8

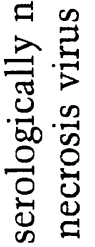

苛穿

式

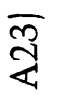

葛 華

疍

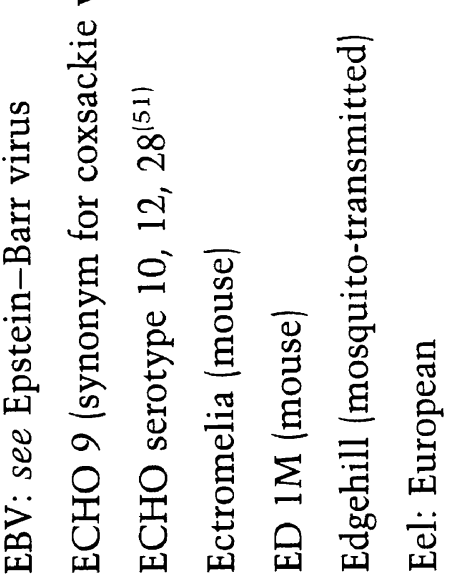




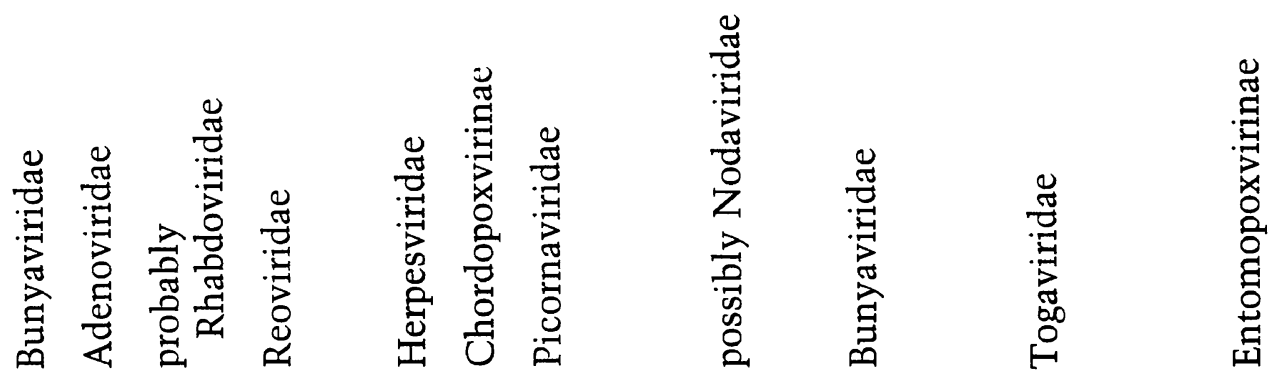
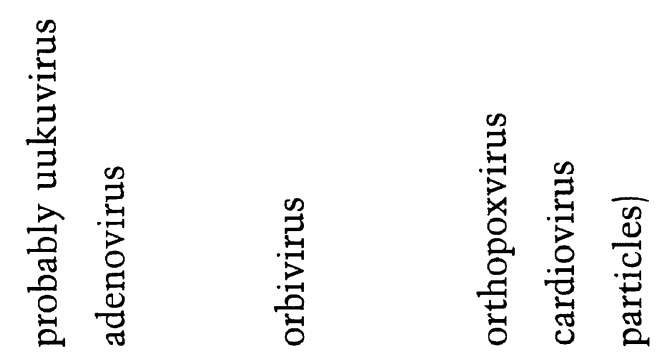

胥

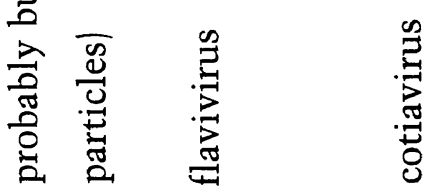

:

苛

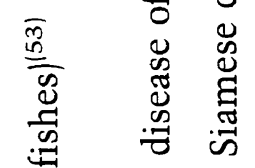

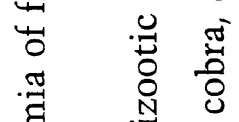

急

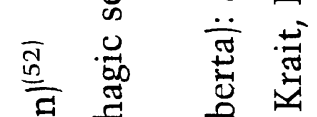

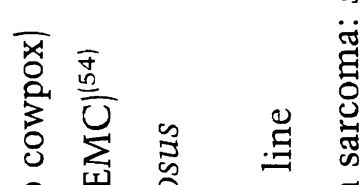

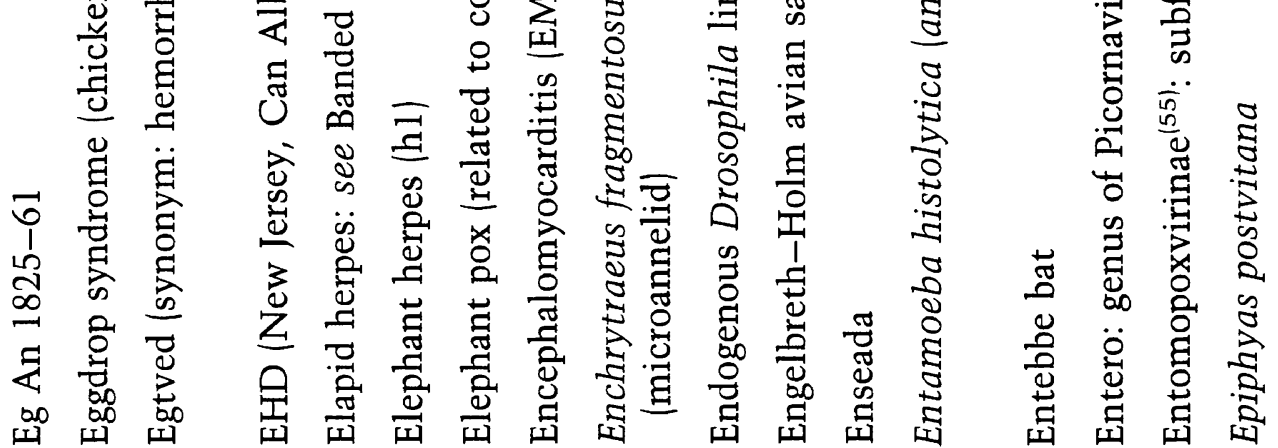

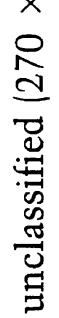

E

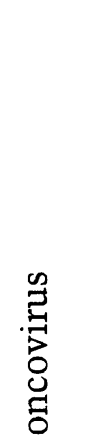

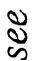

包

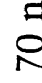

를

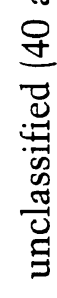

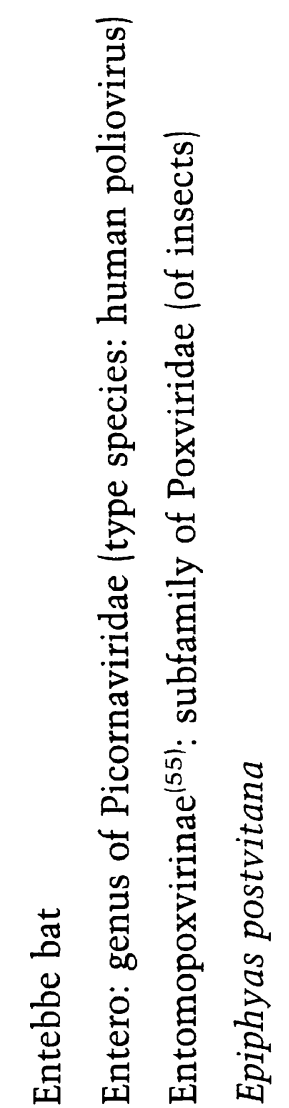



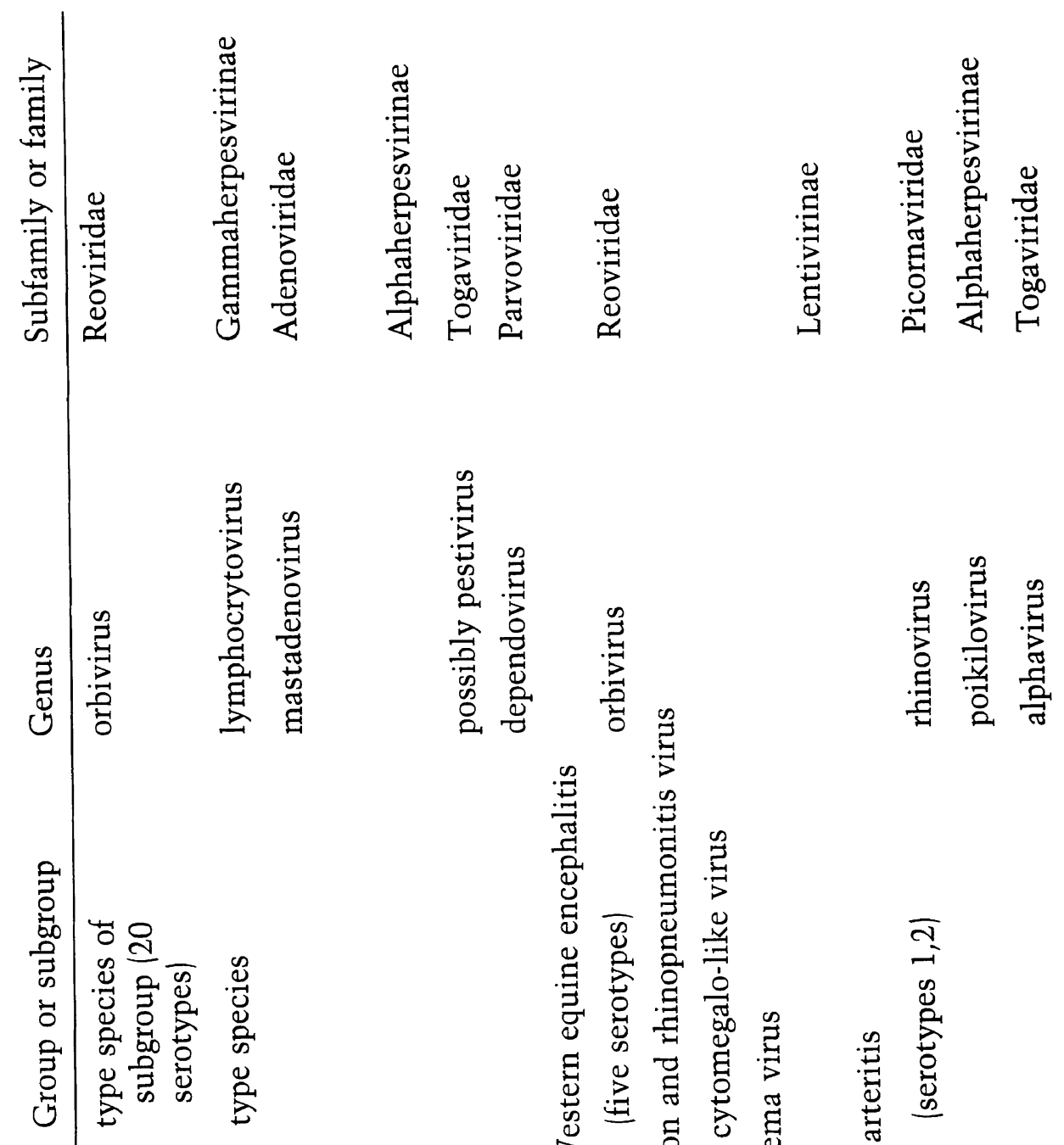

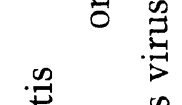
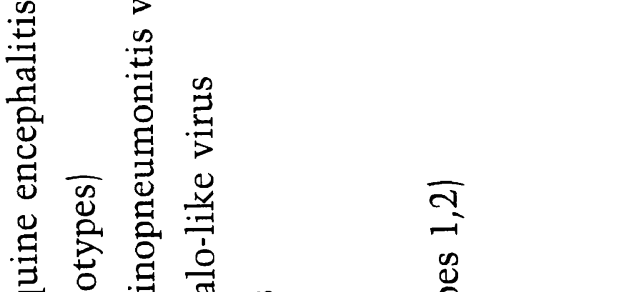

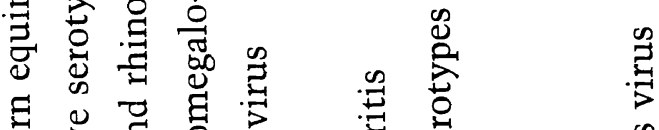

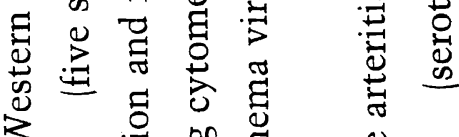



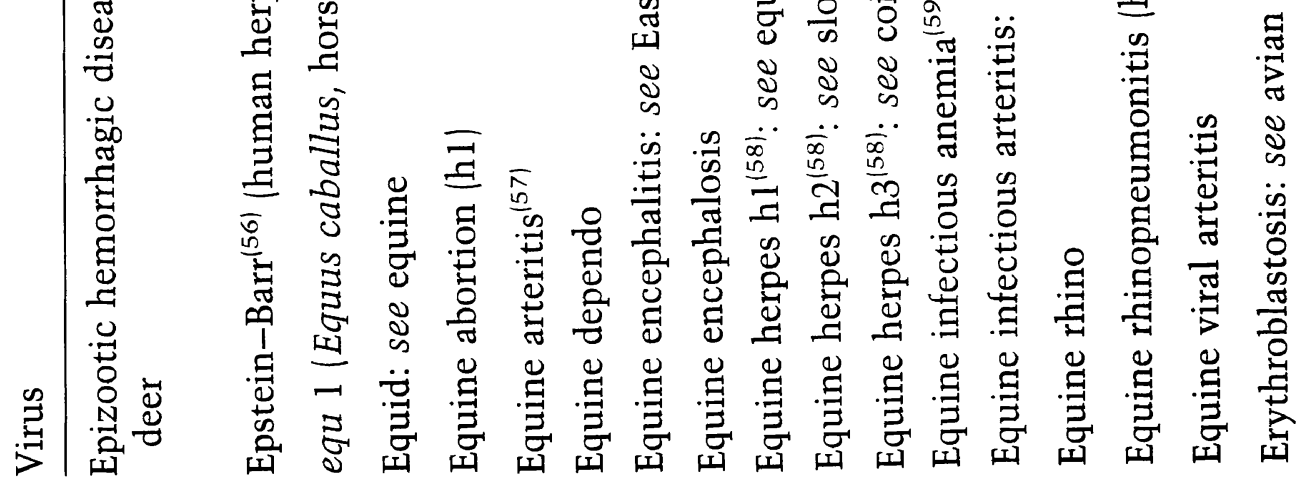



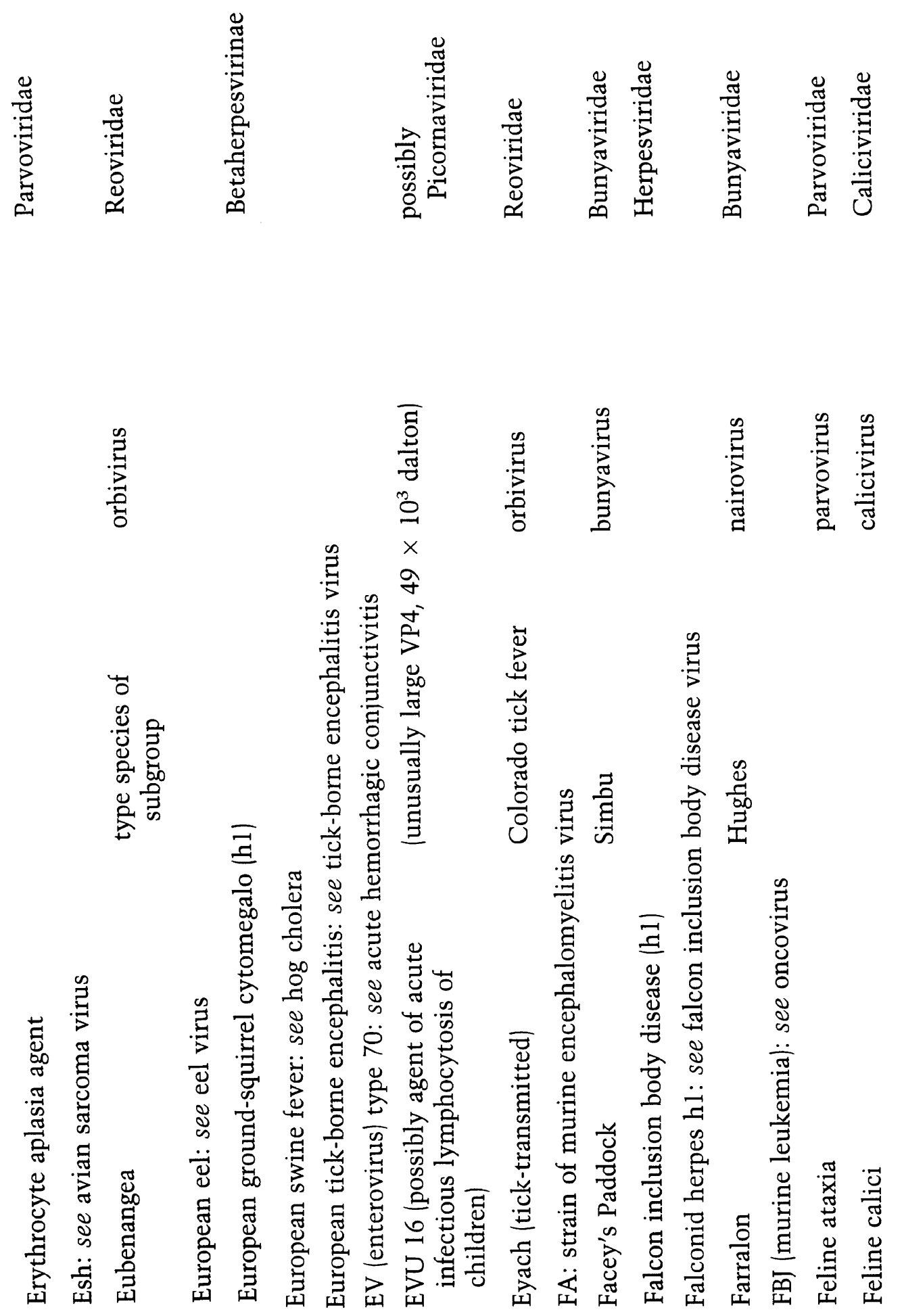


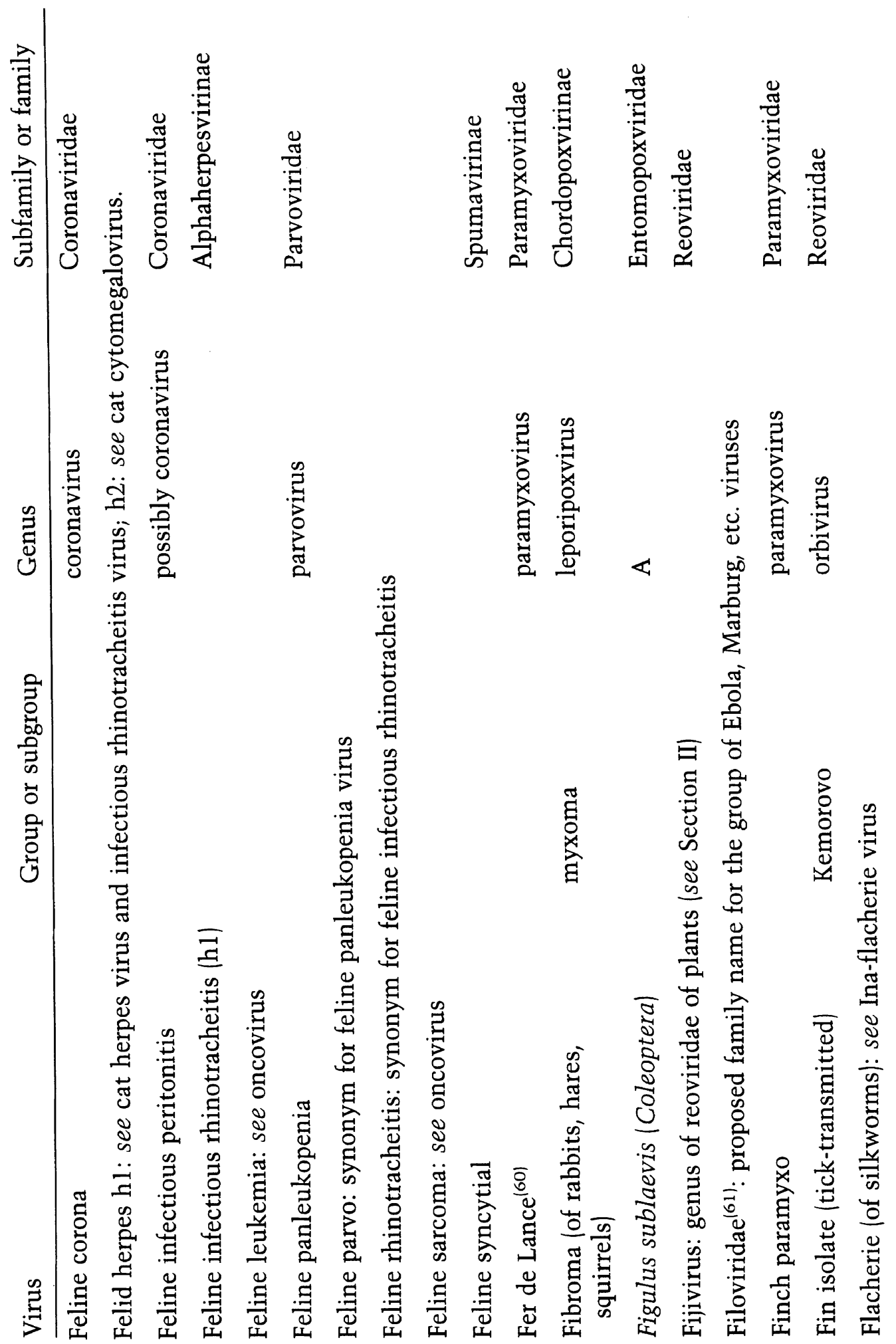



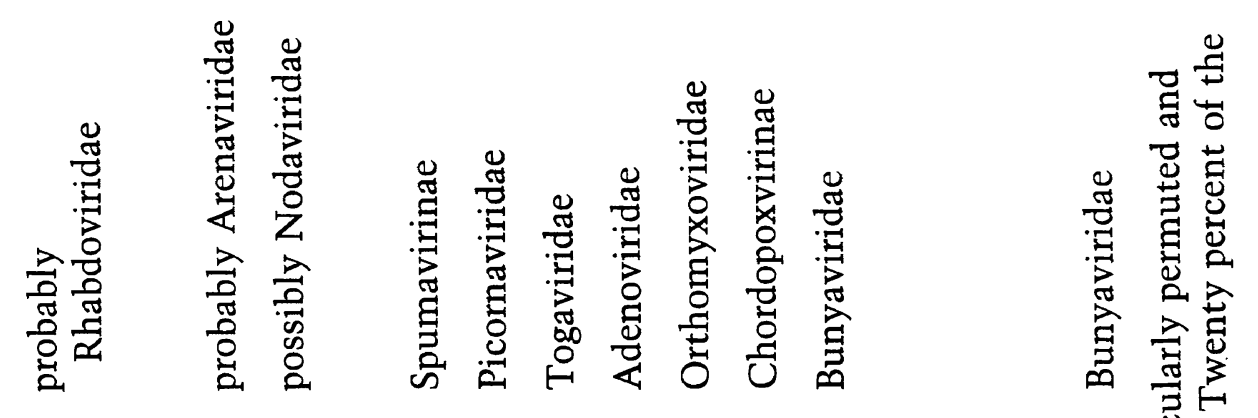


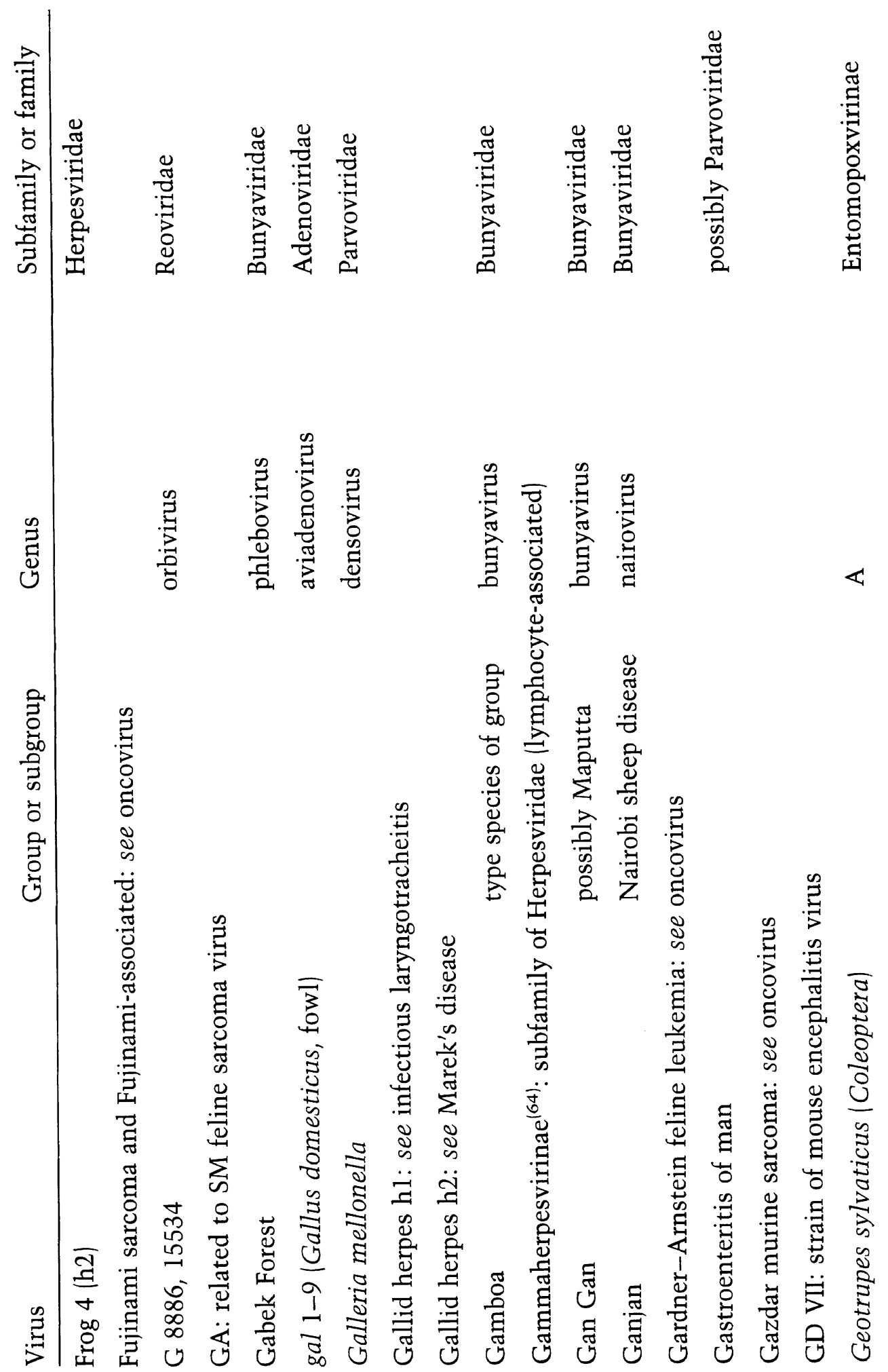



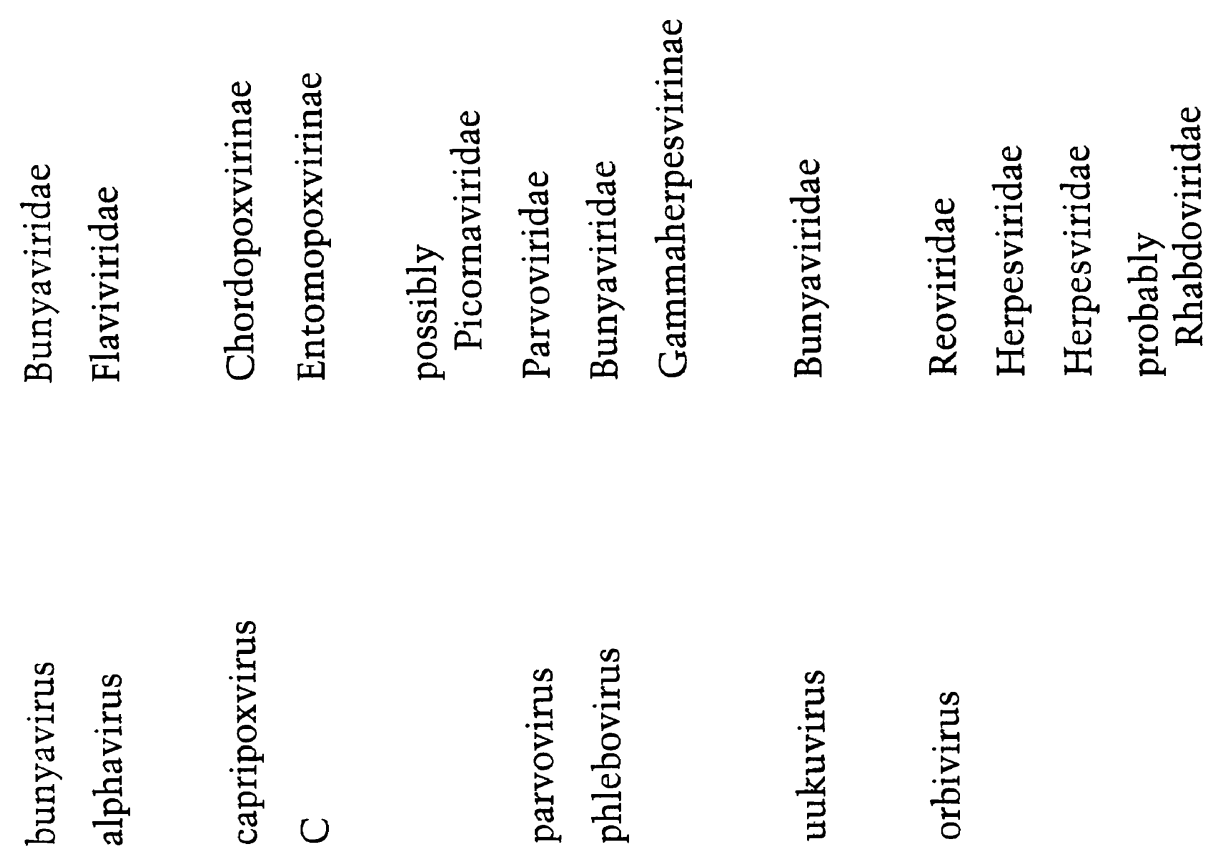

荥

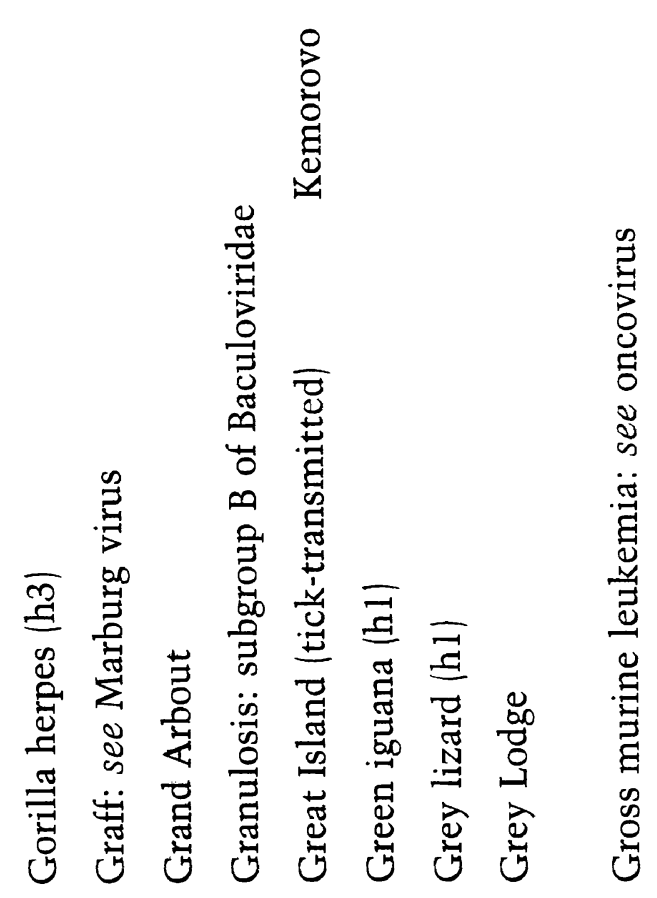




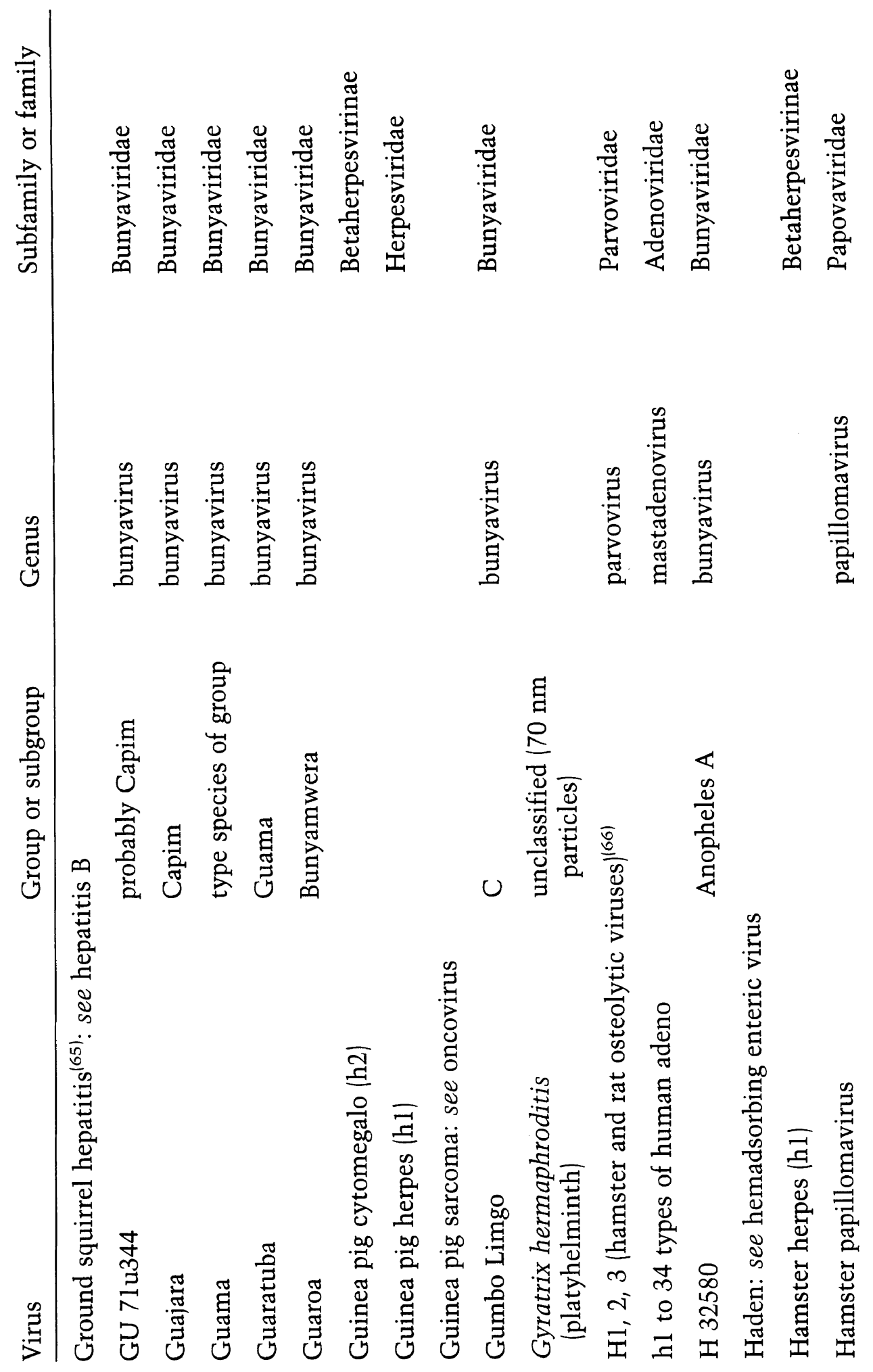




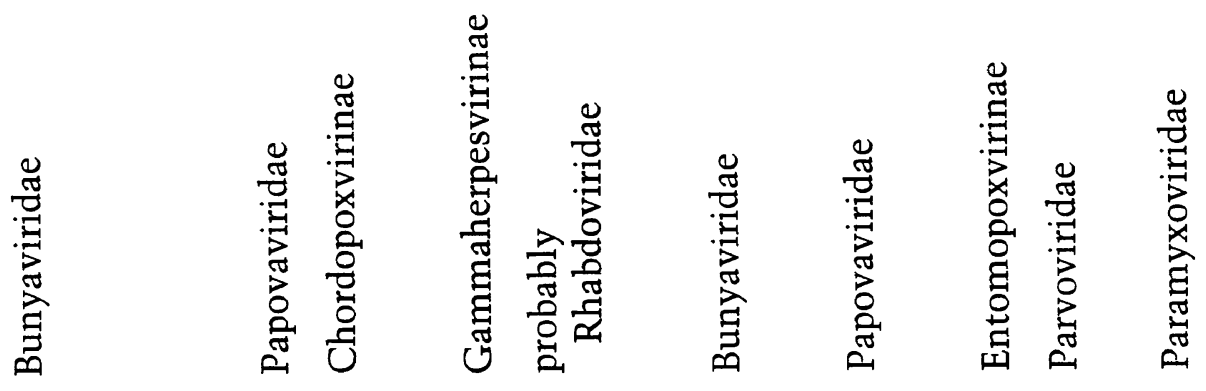

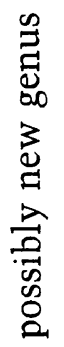

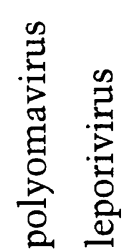

范
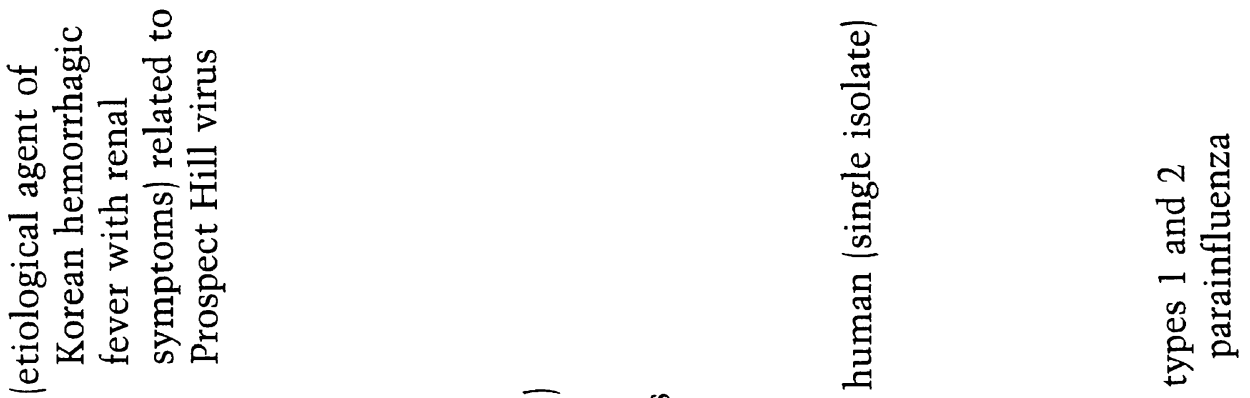

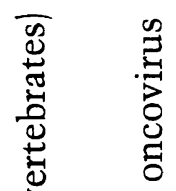

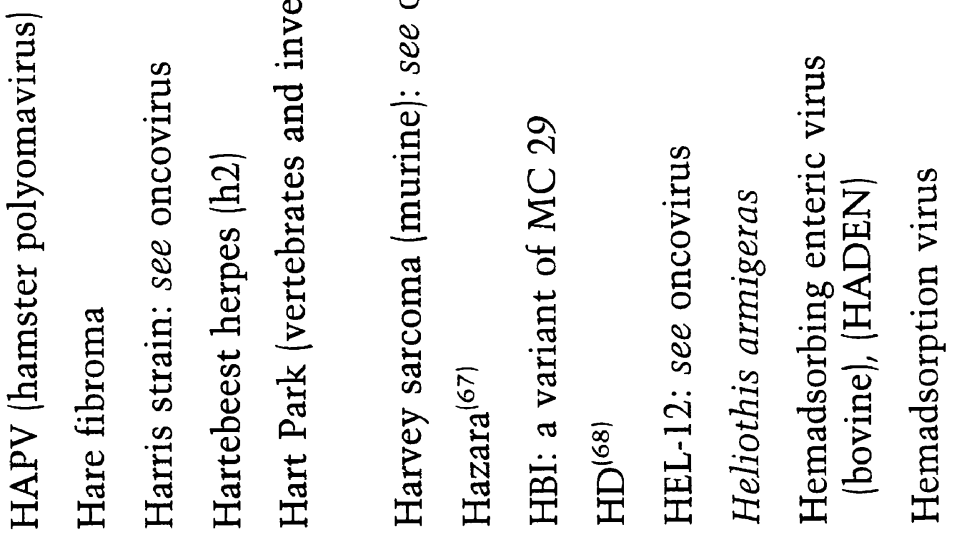




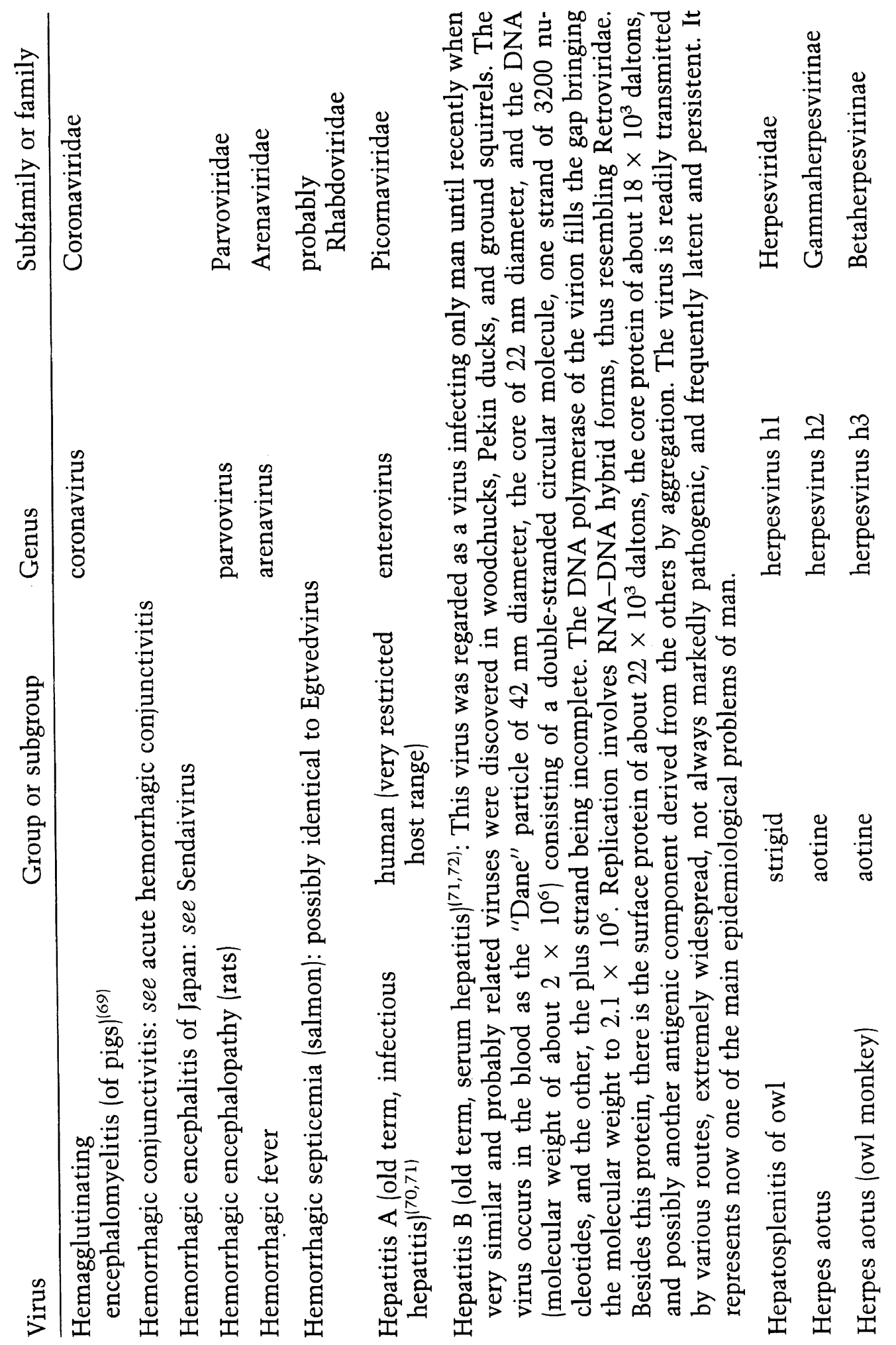



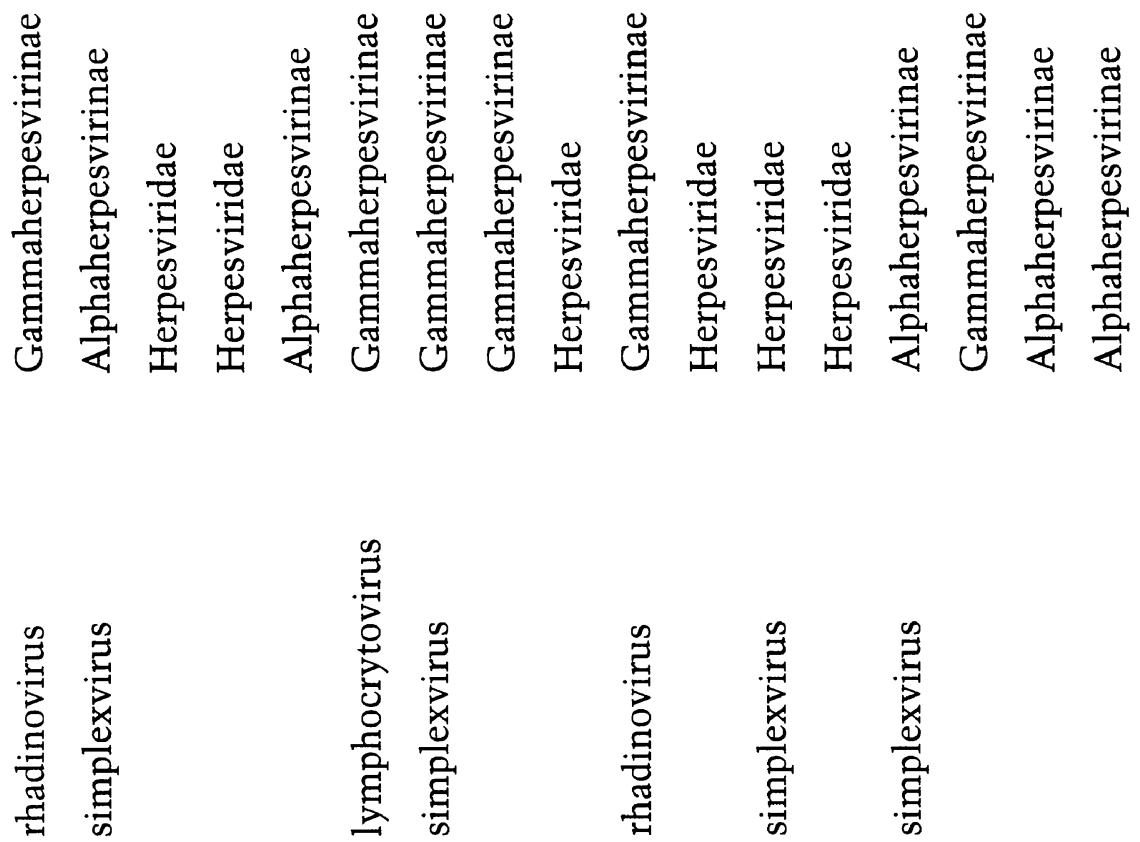

হ্త

초

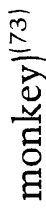

苛

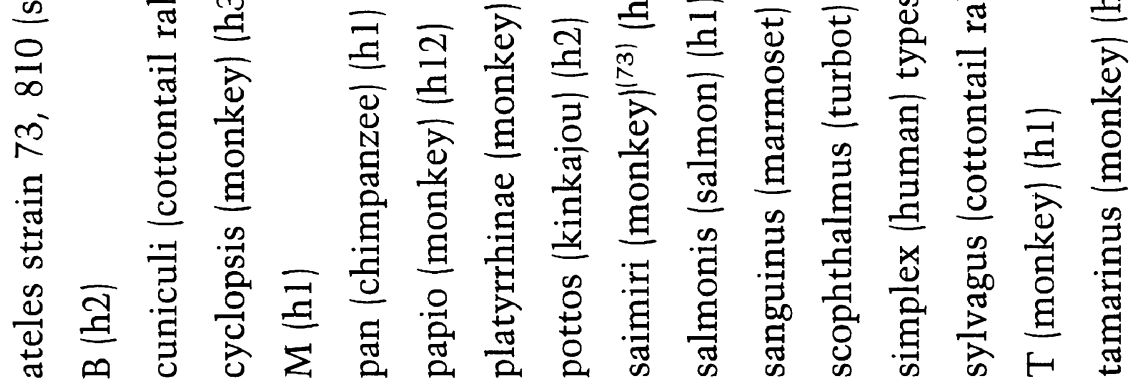

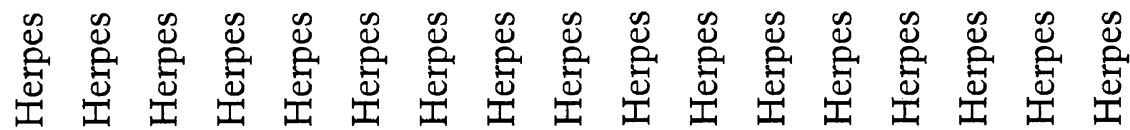




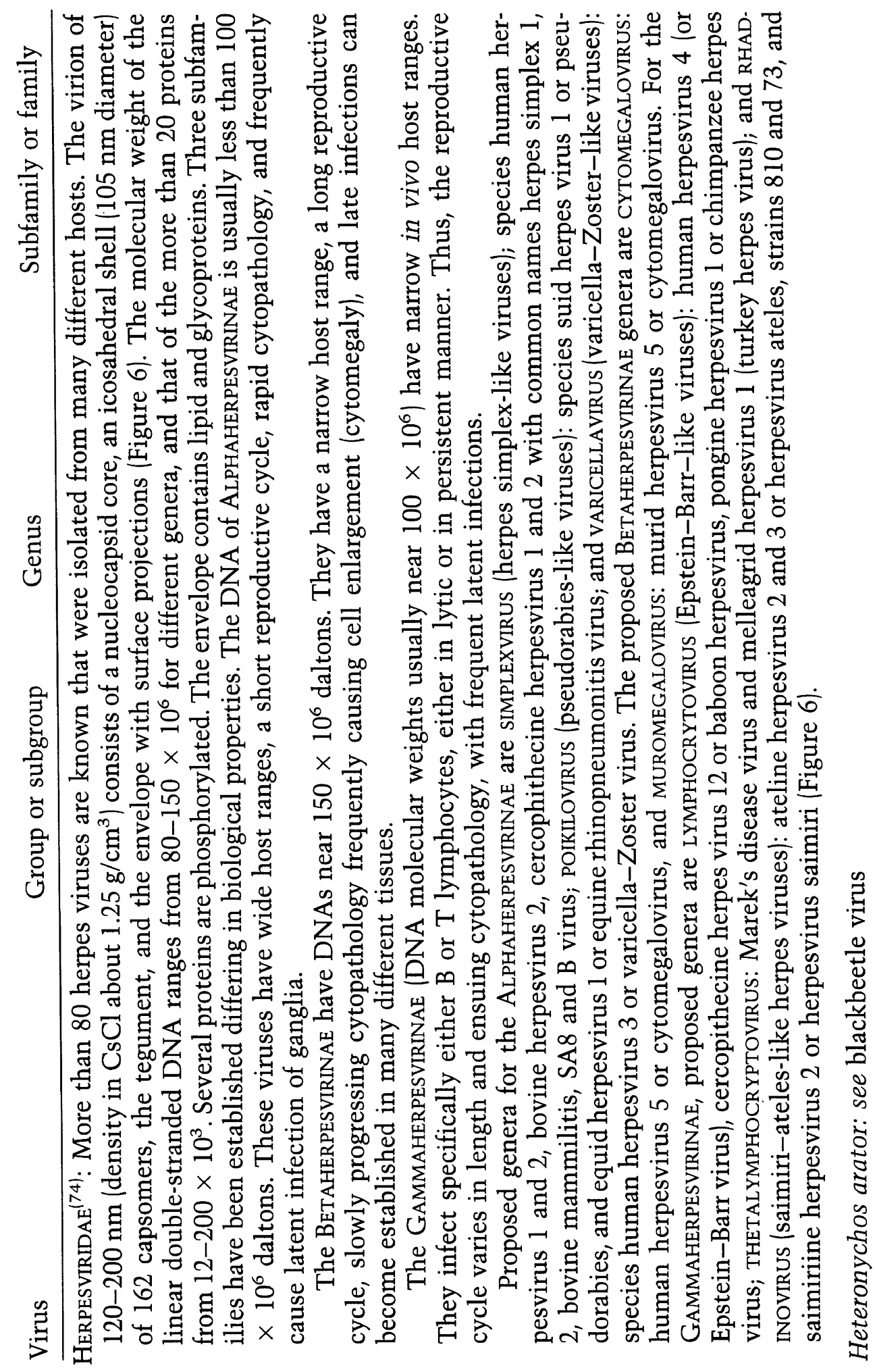



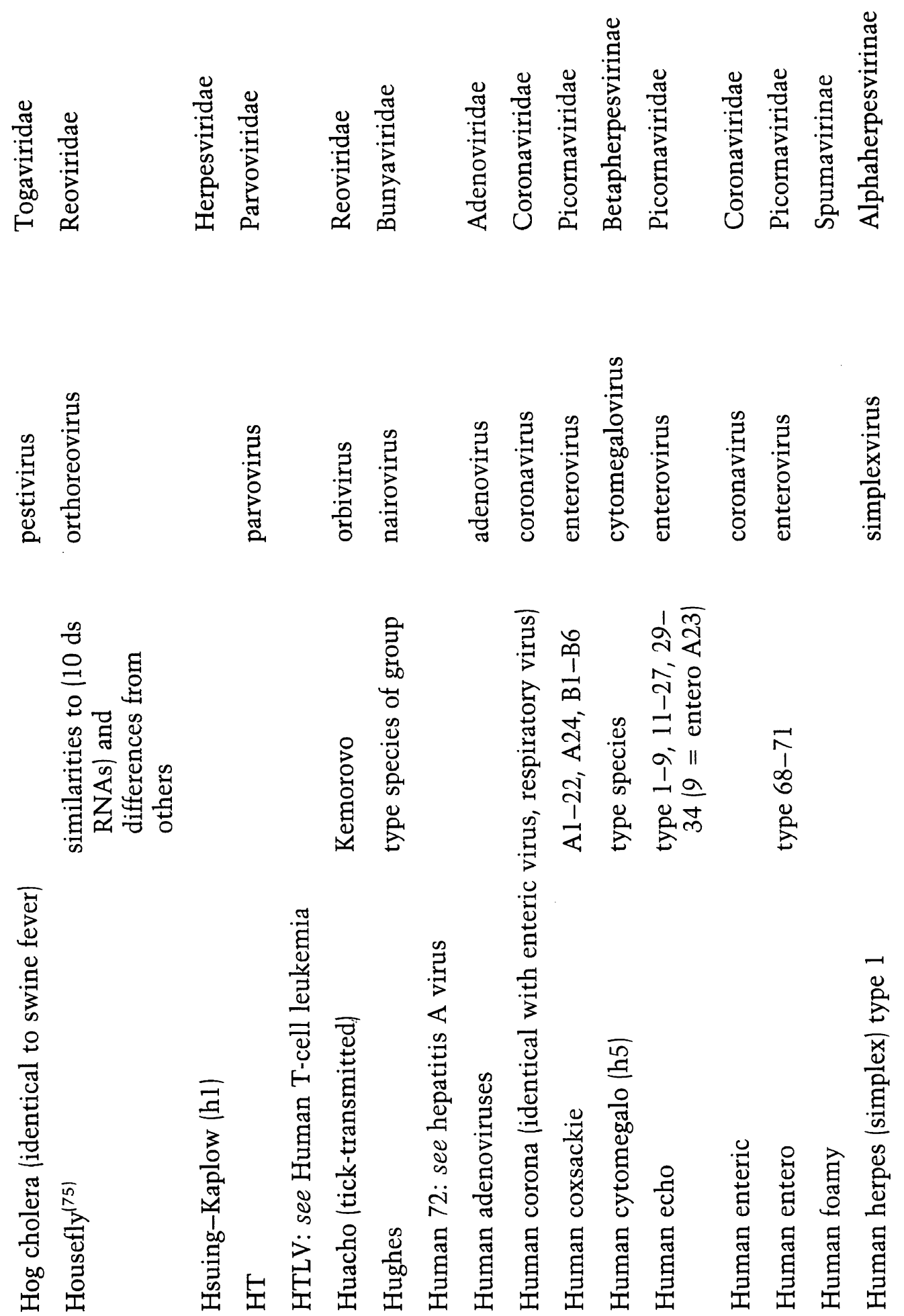


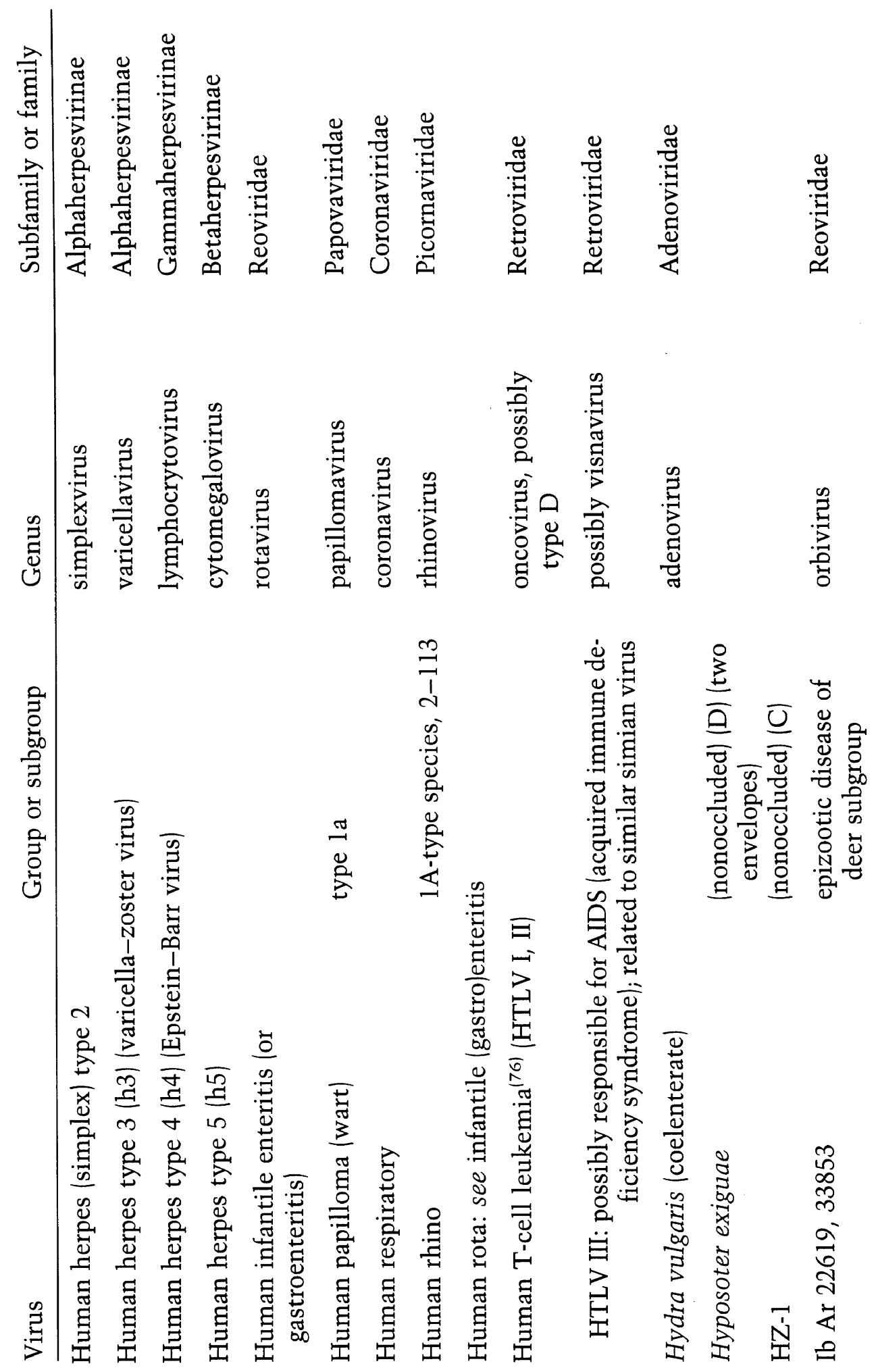




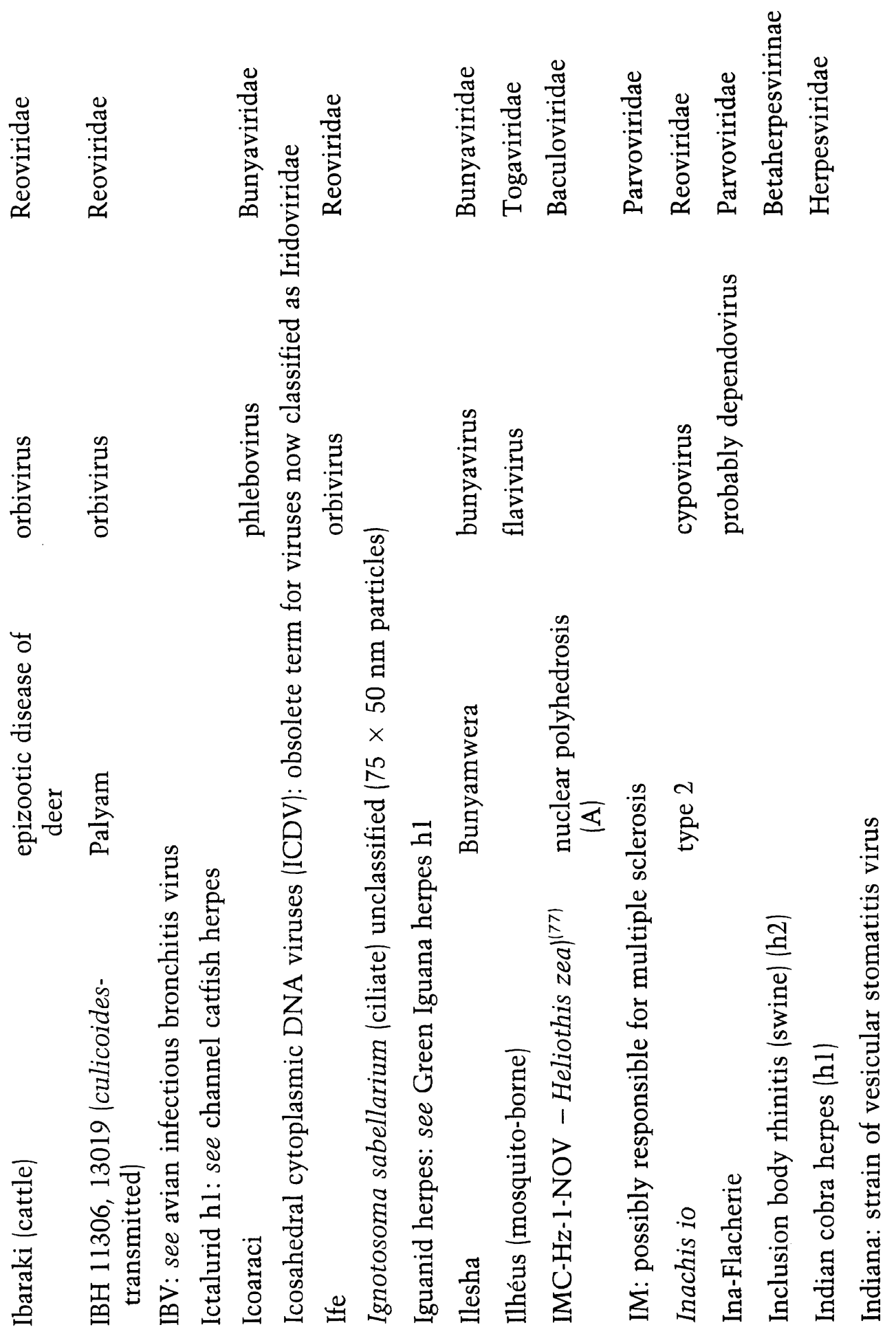



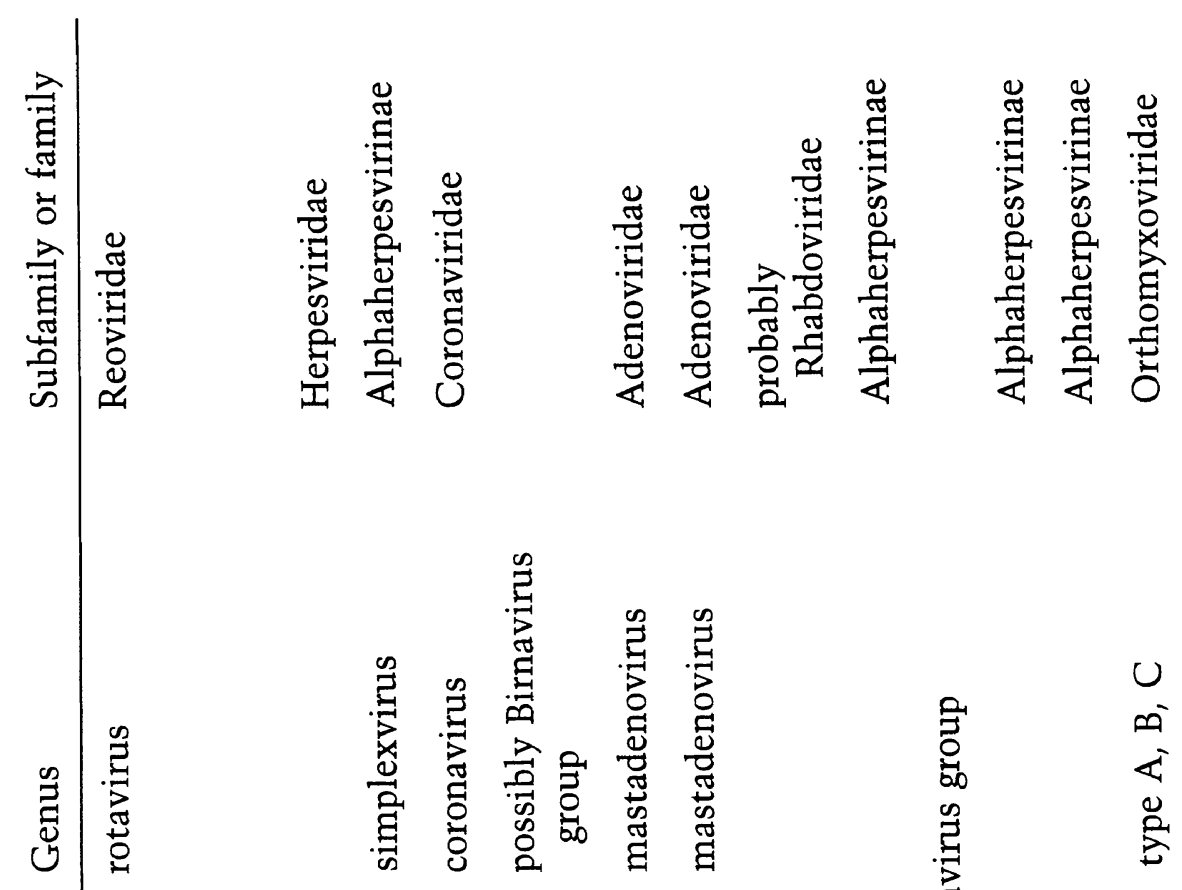

. 


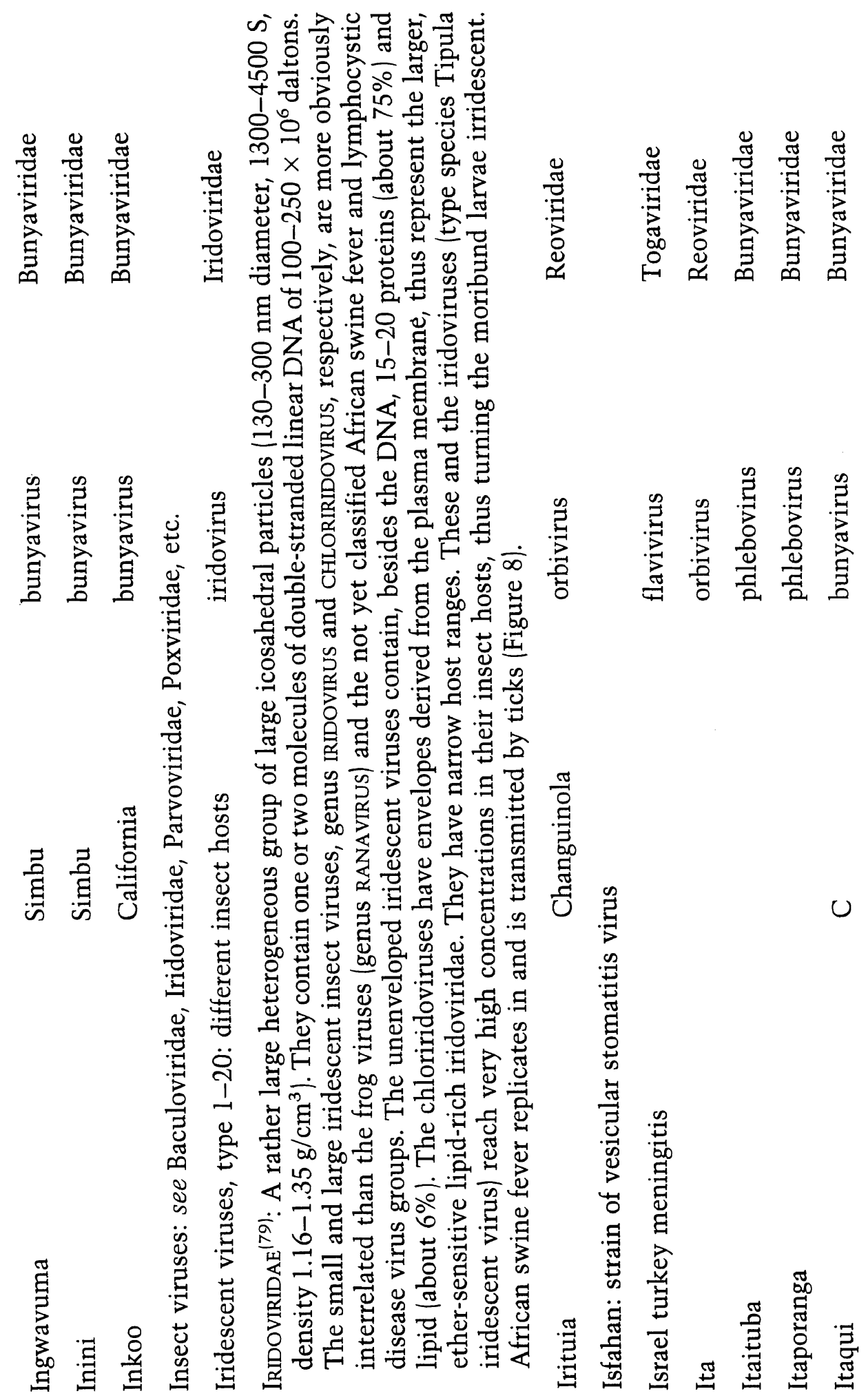




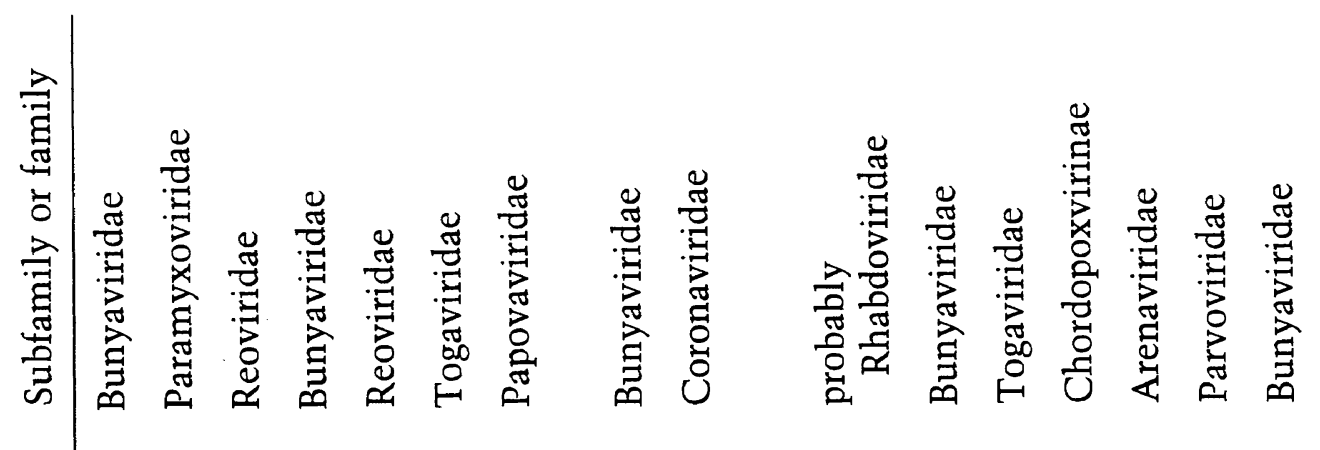

|

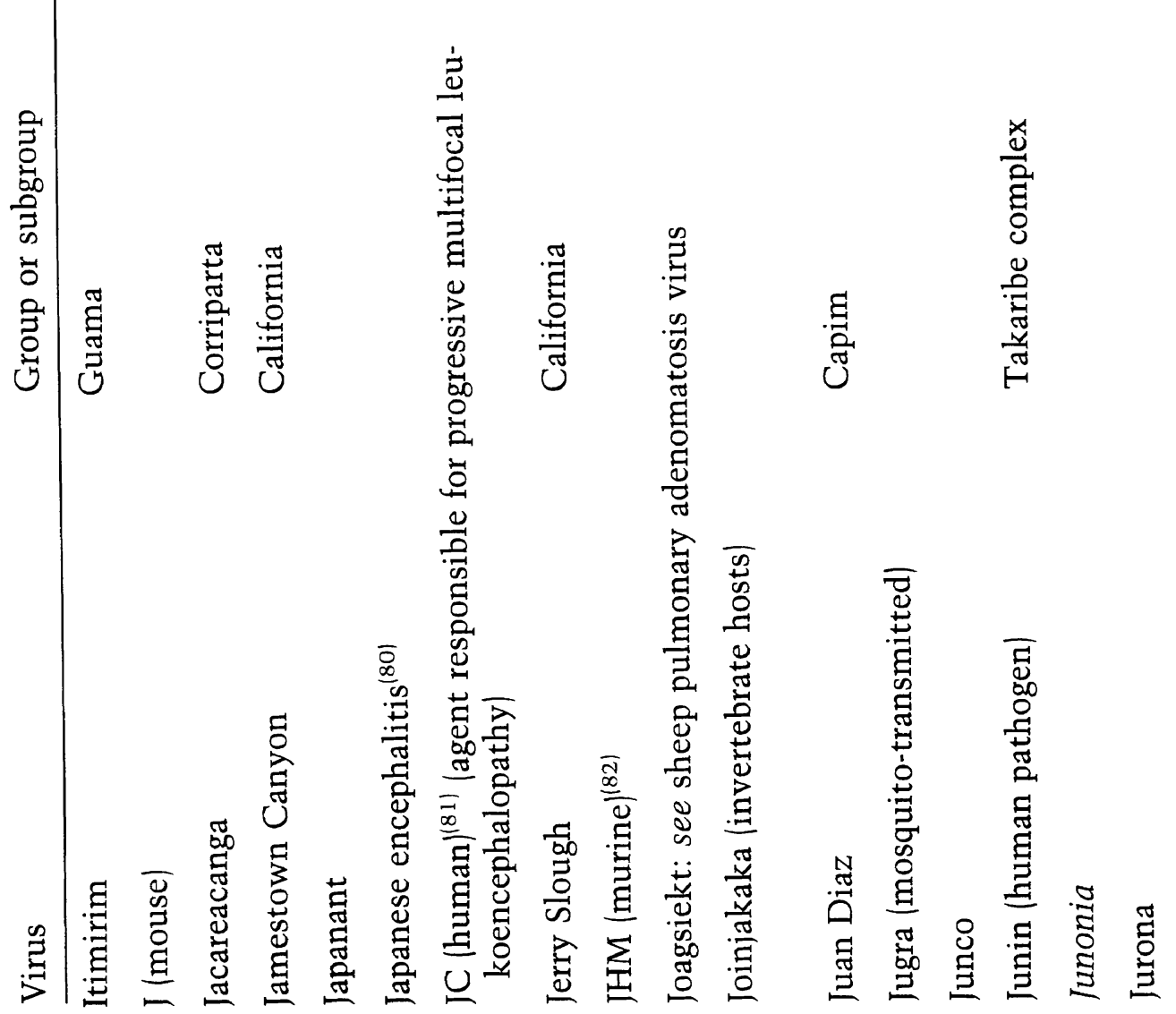



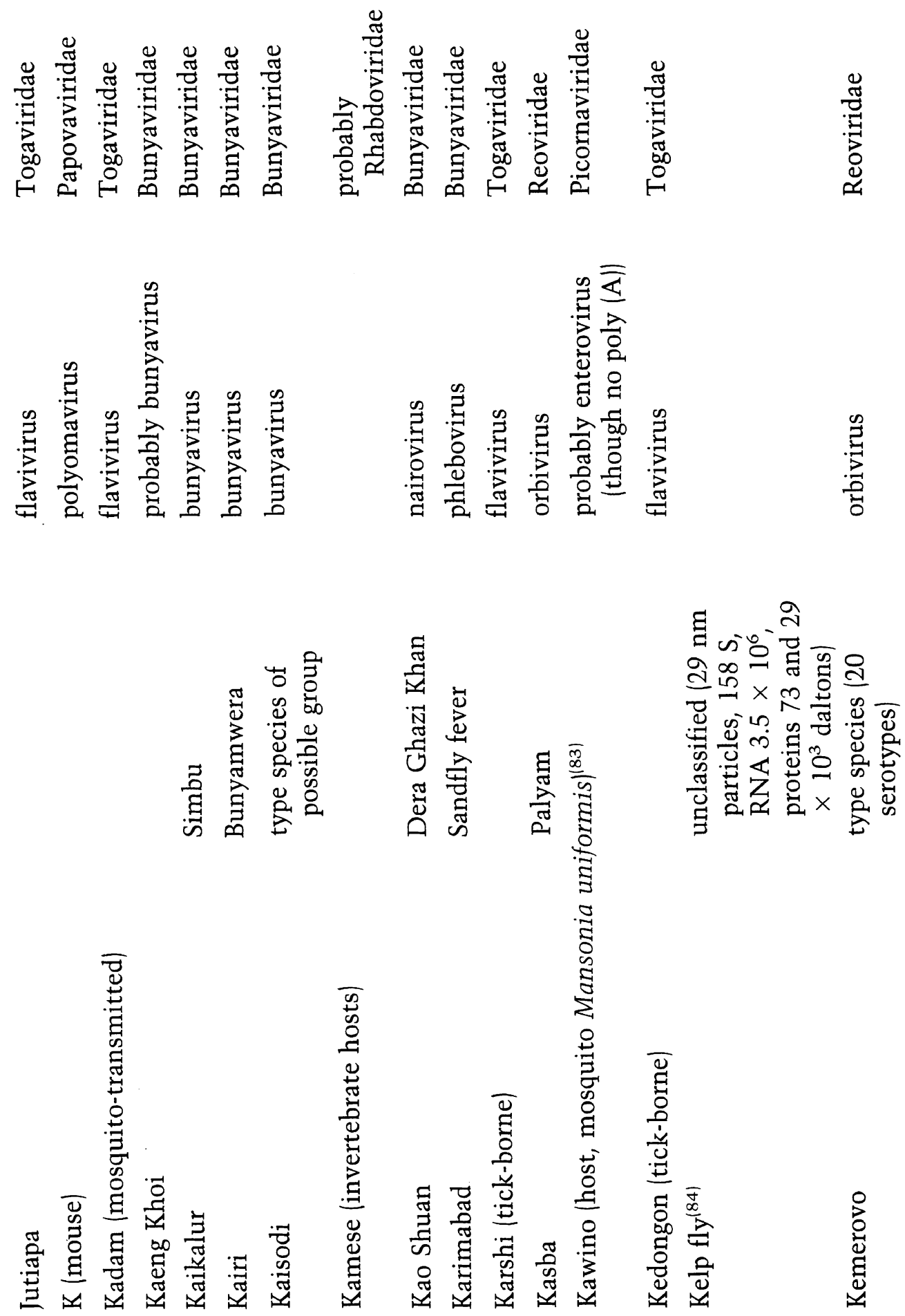


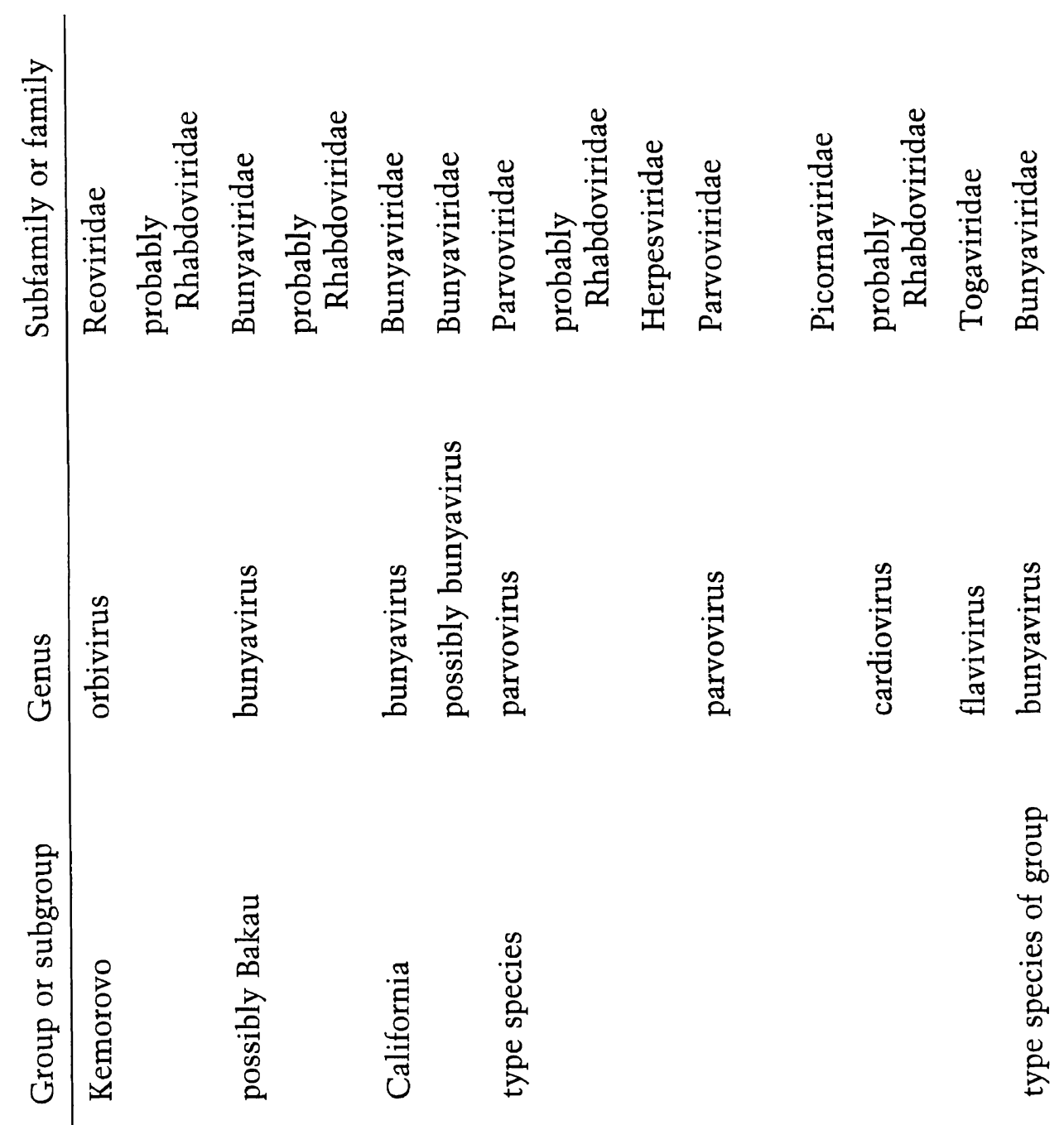


营
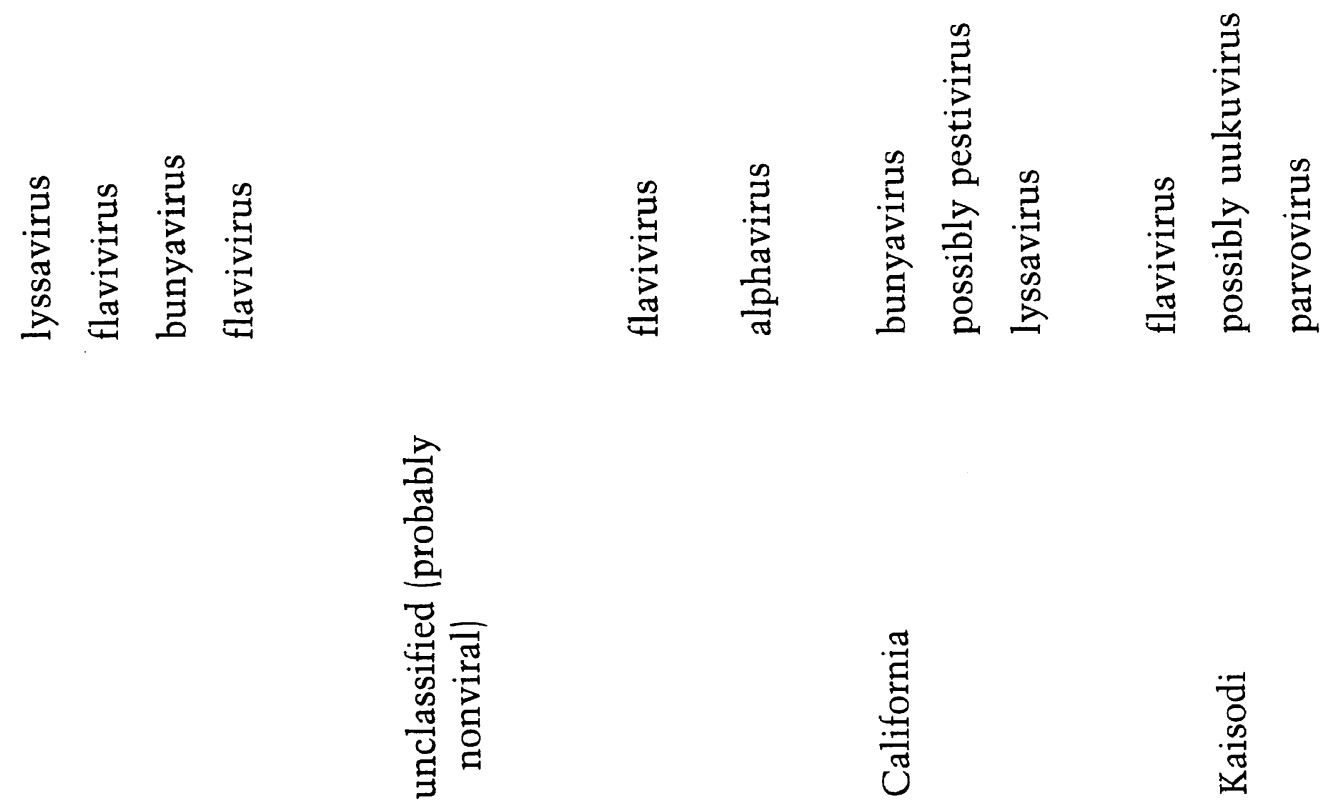

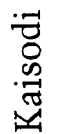

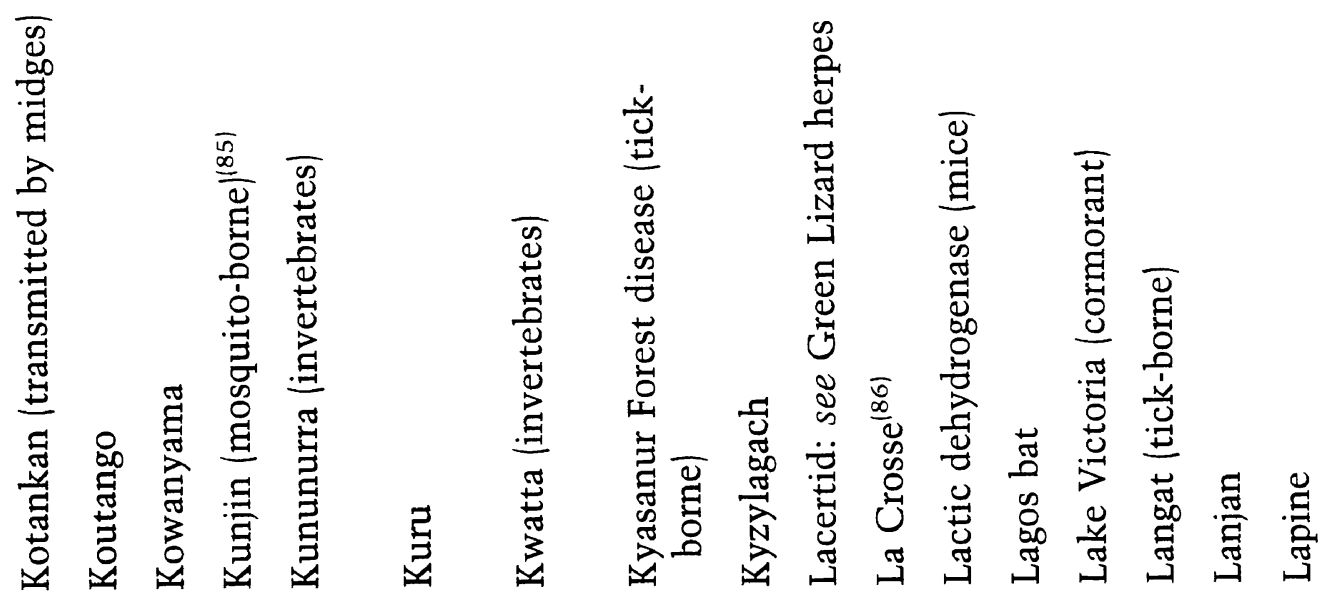



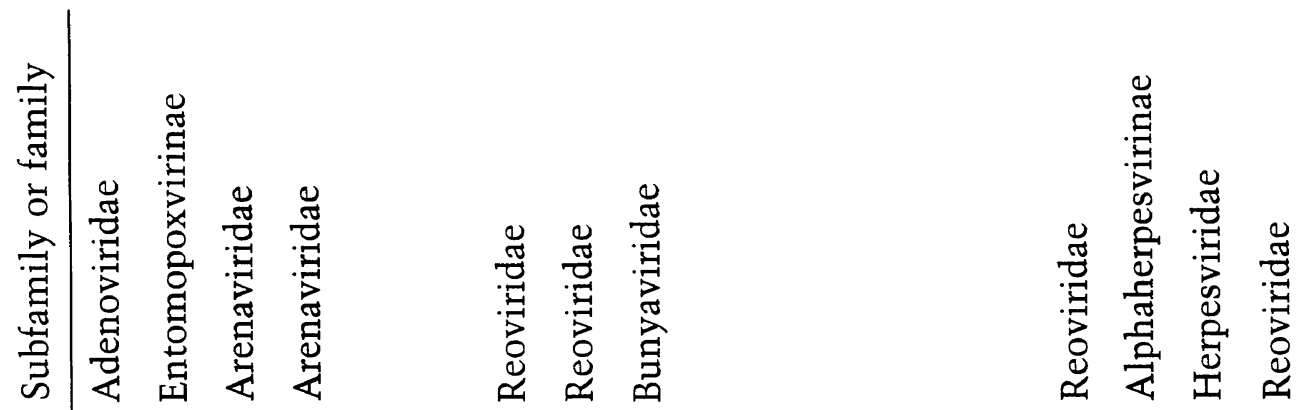

总
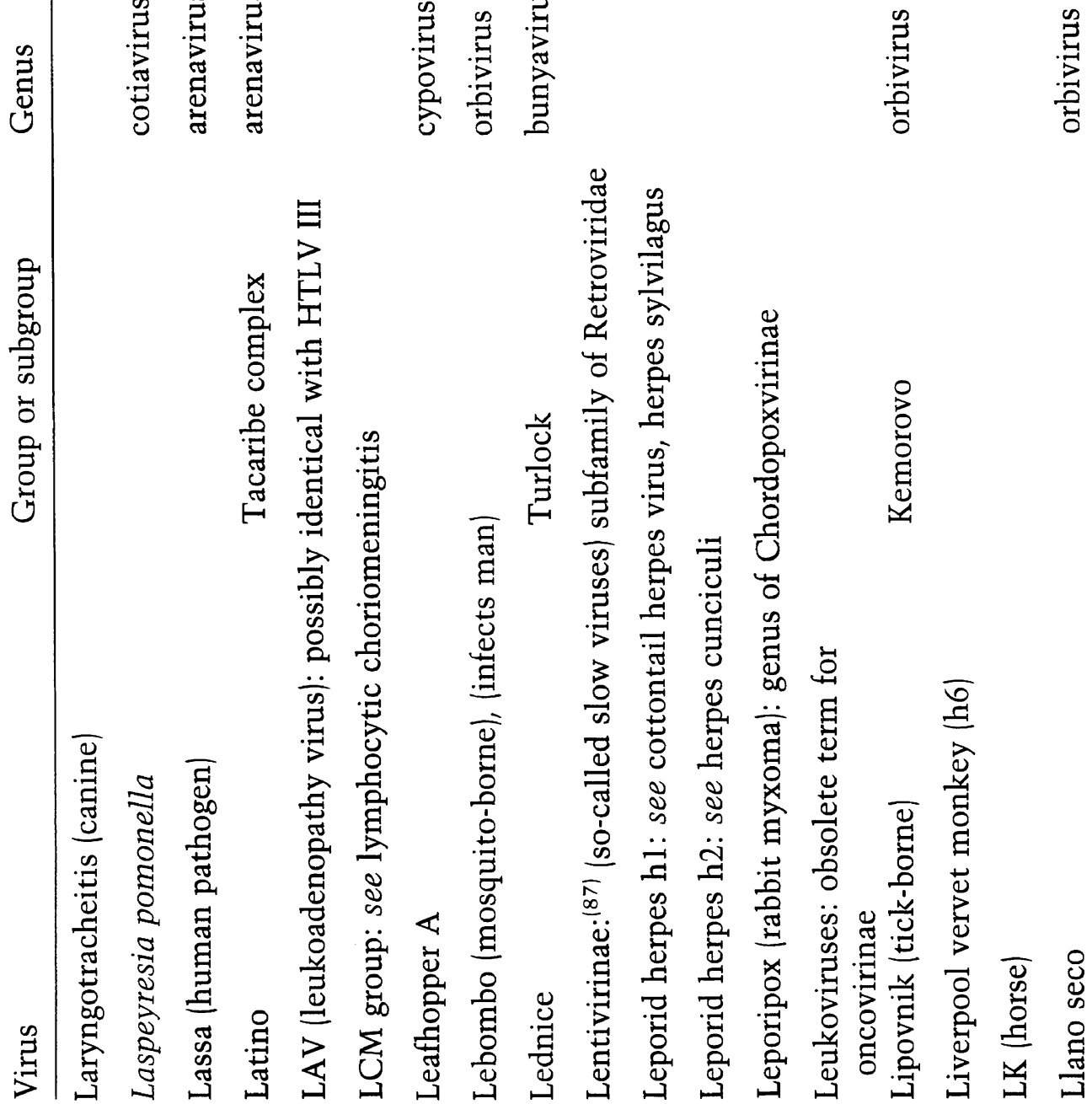


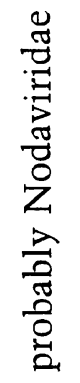
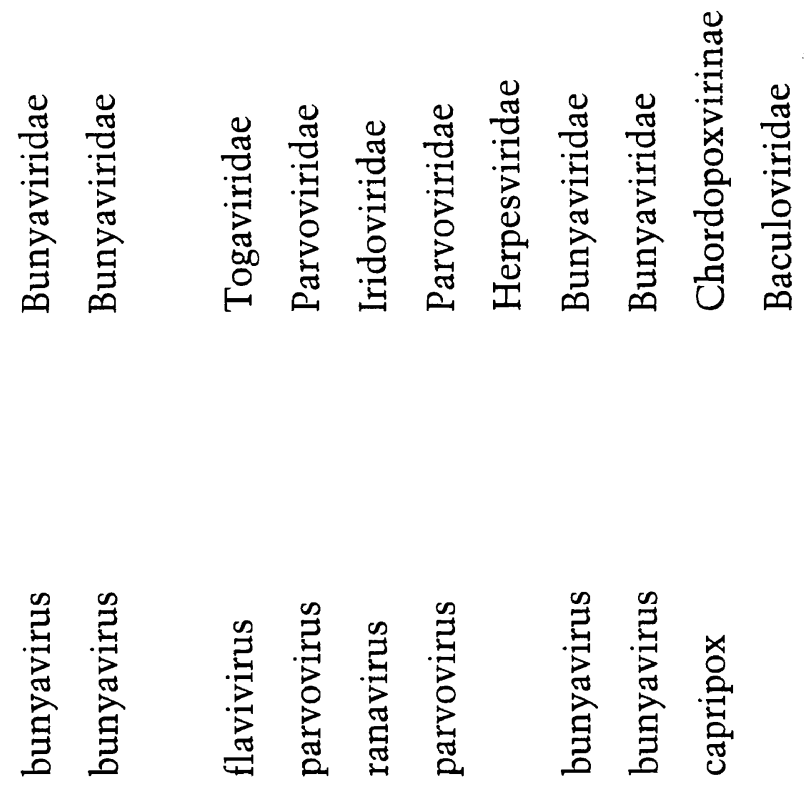

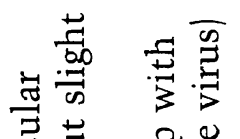

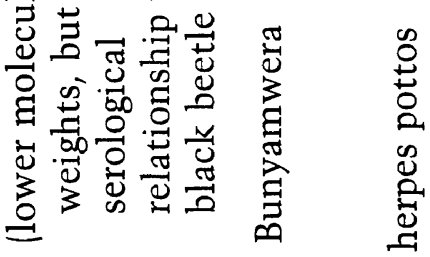

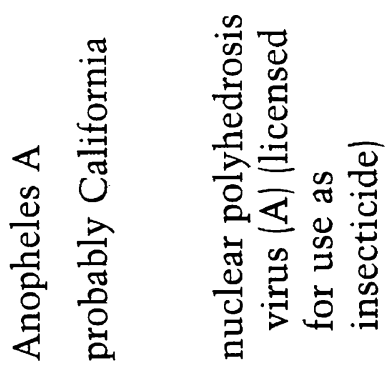

岂

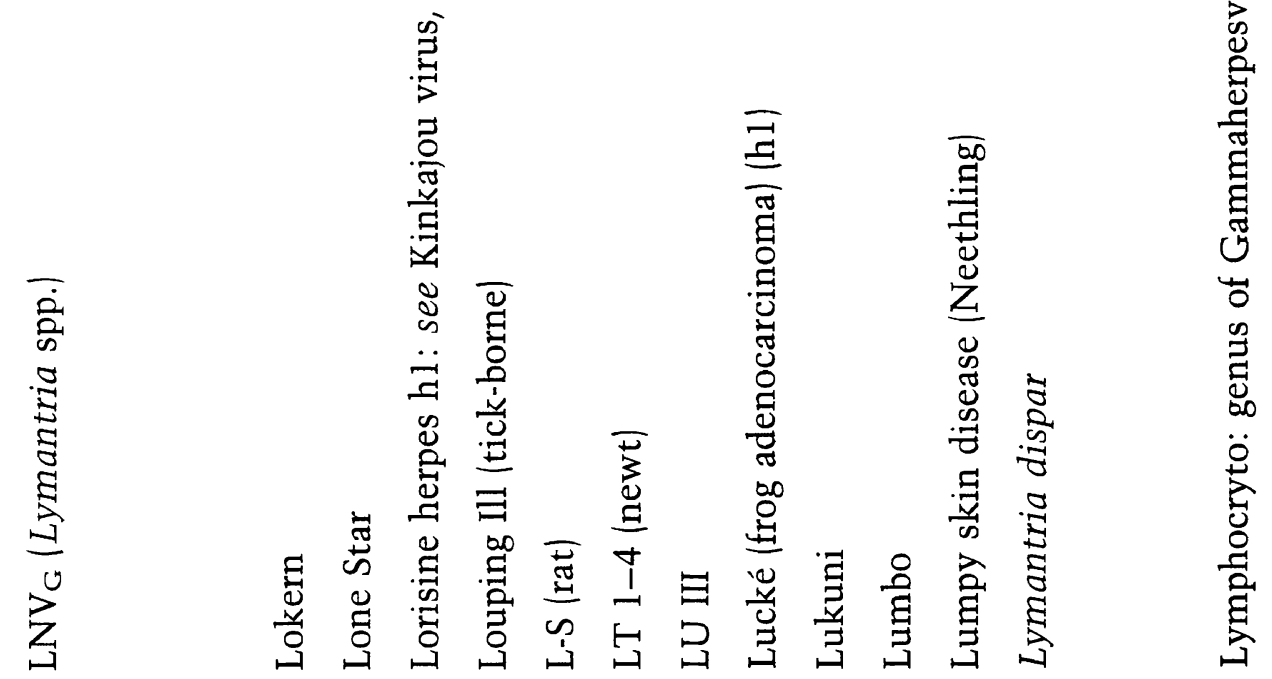




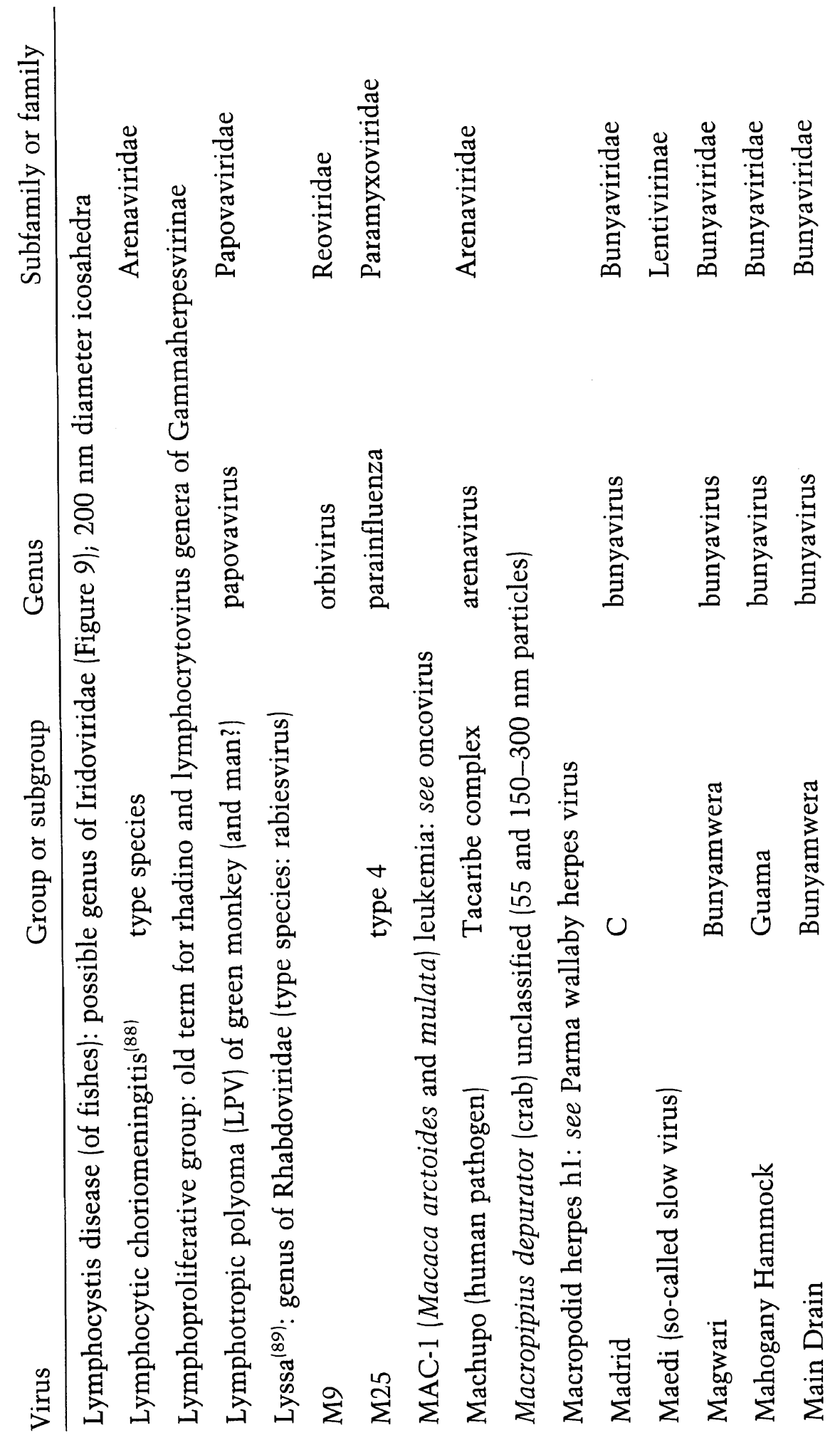



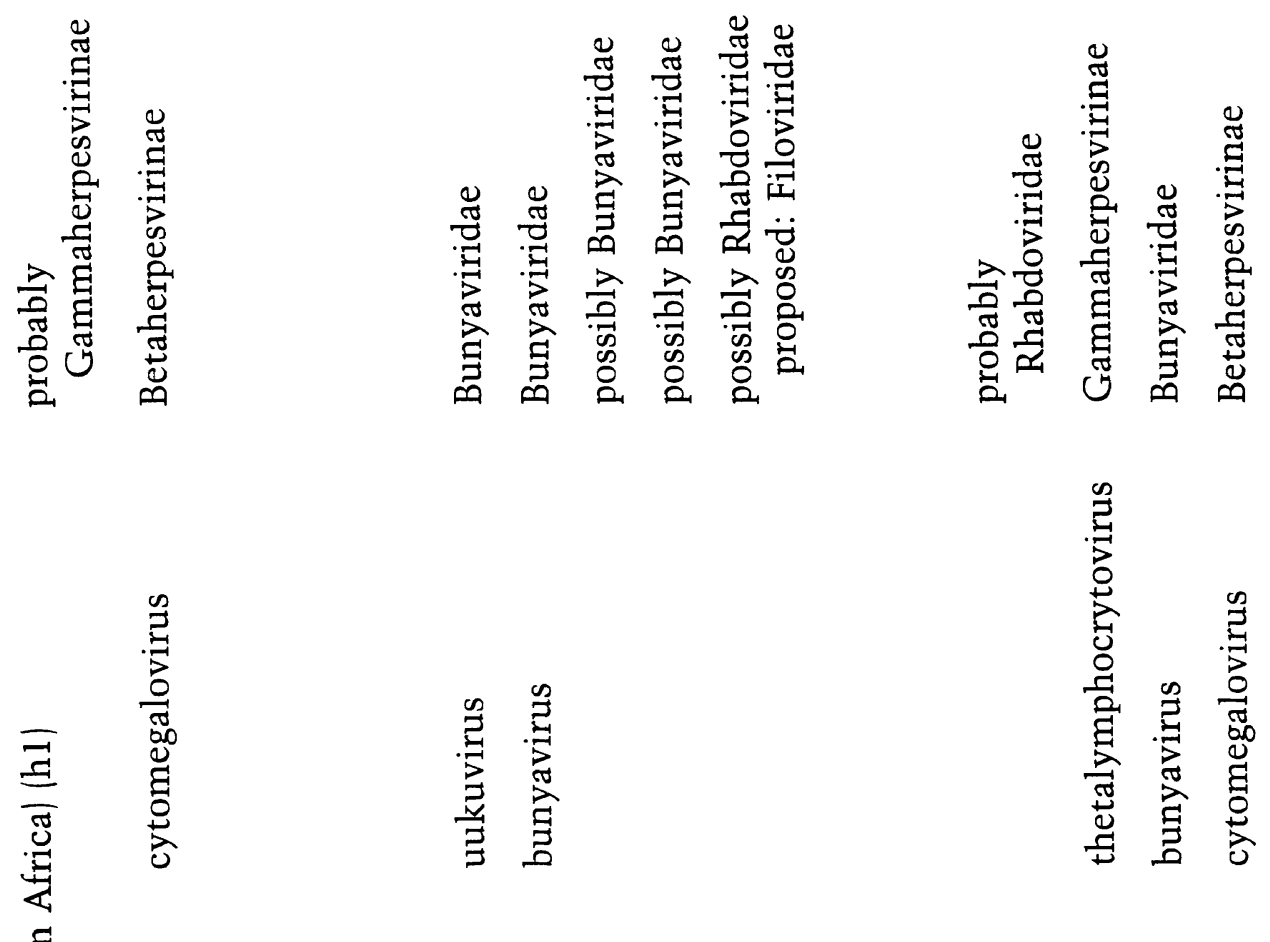

$\Xi$

ن

过

莺

ำ

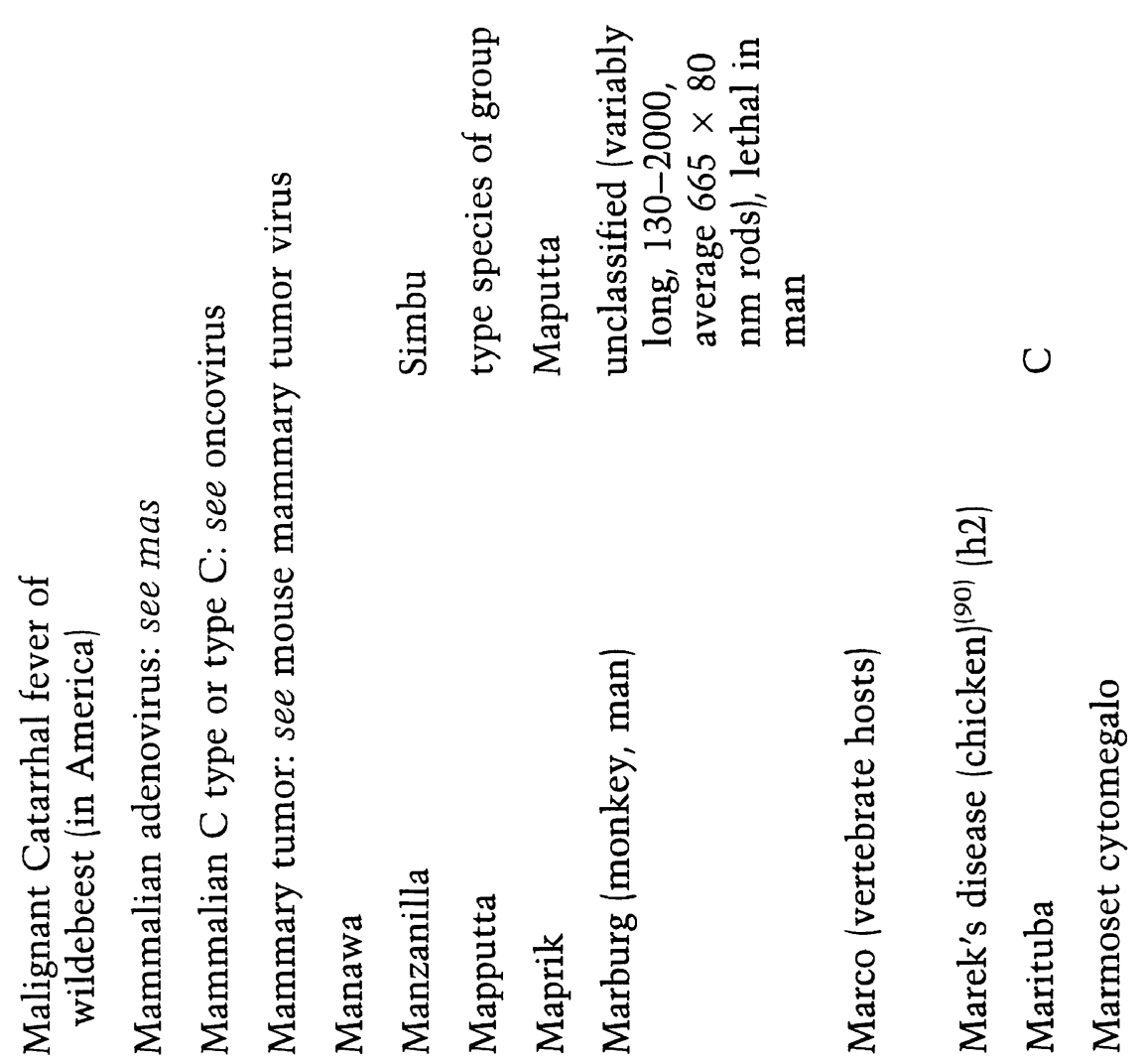




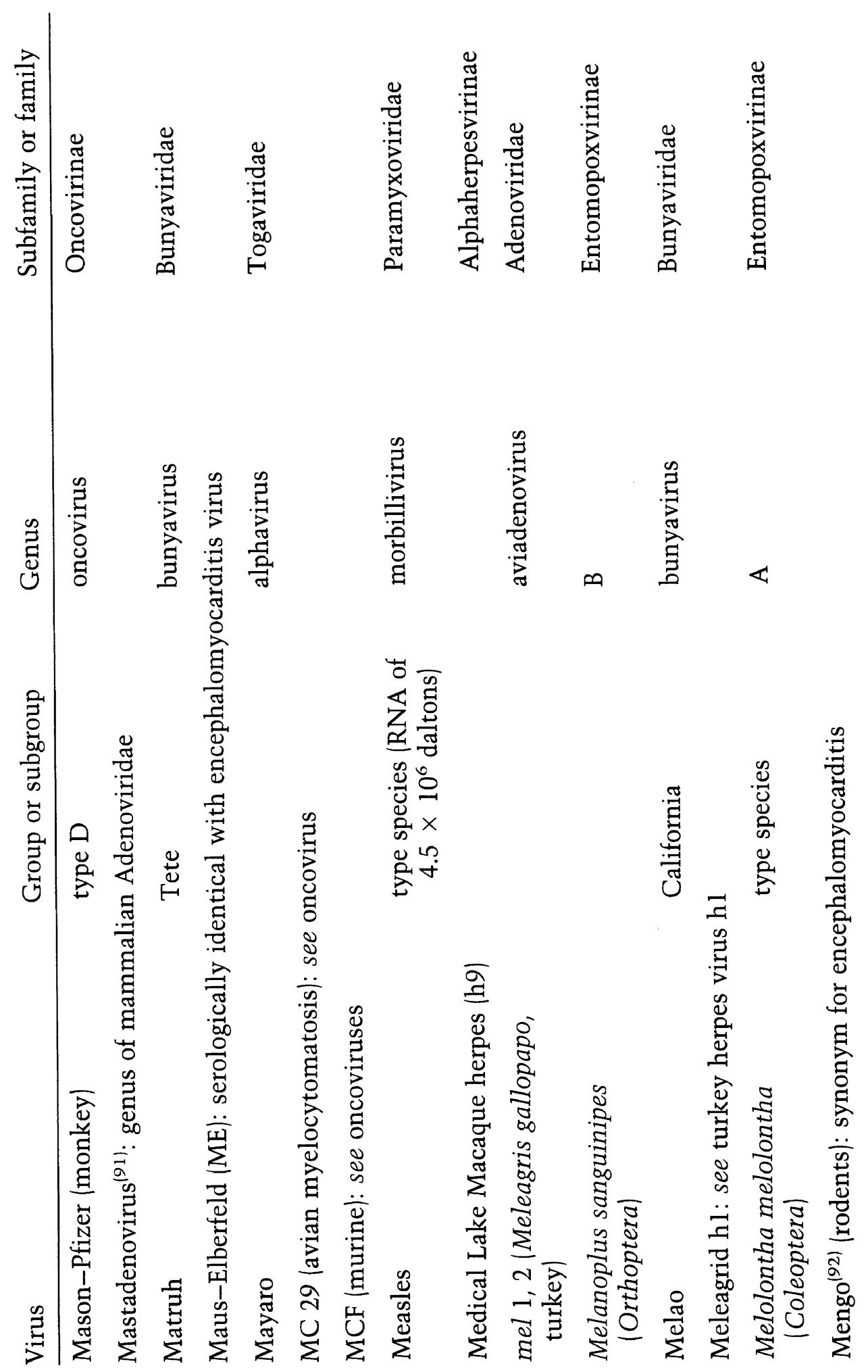



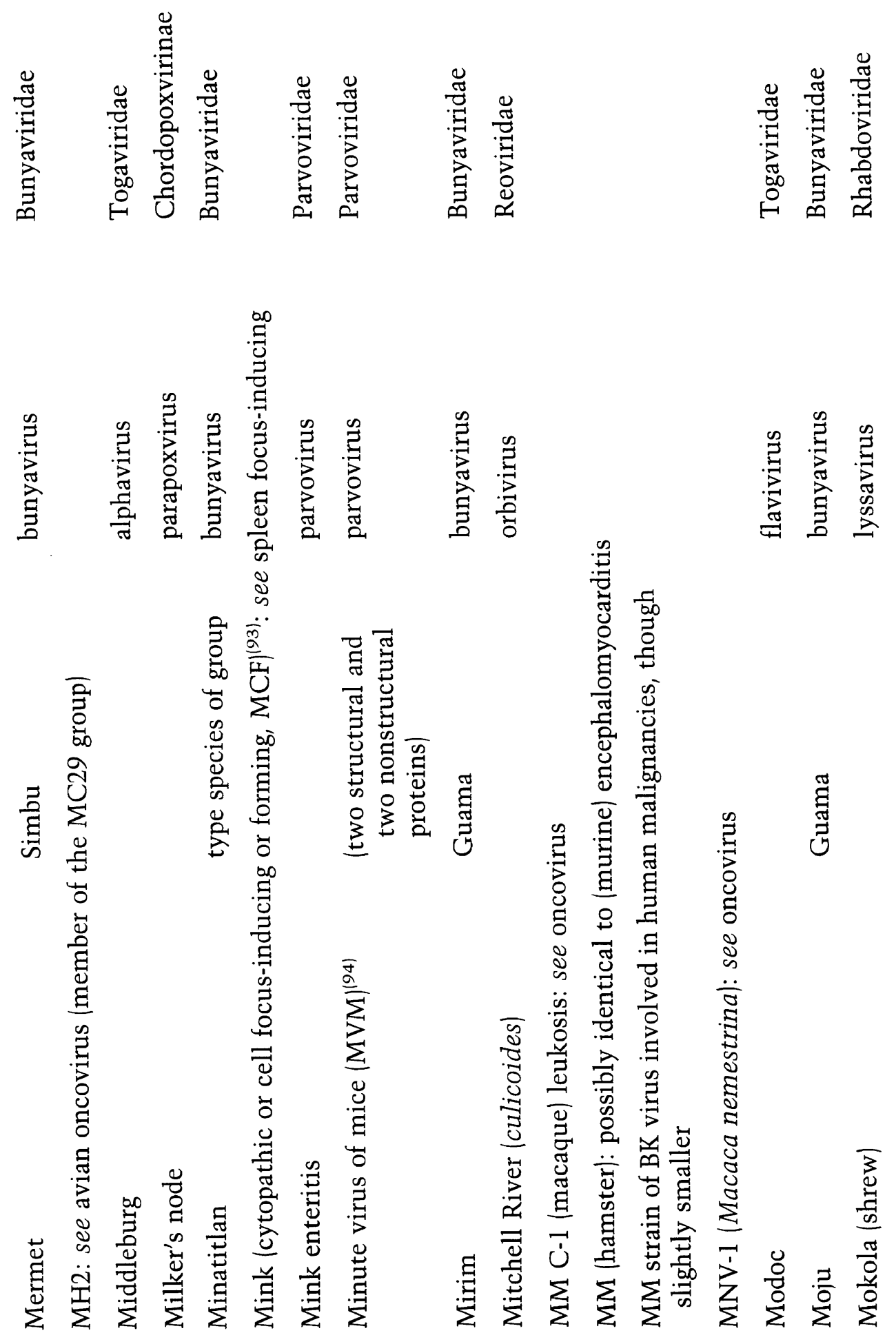


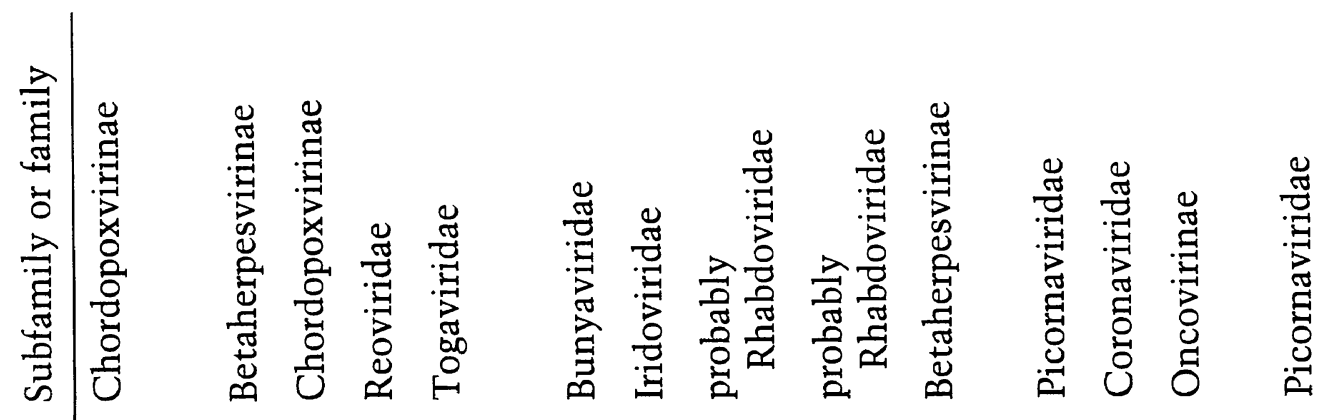

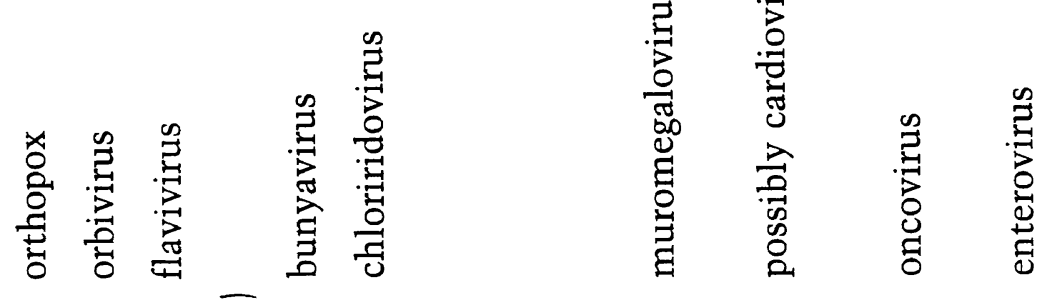

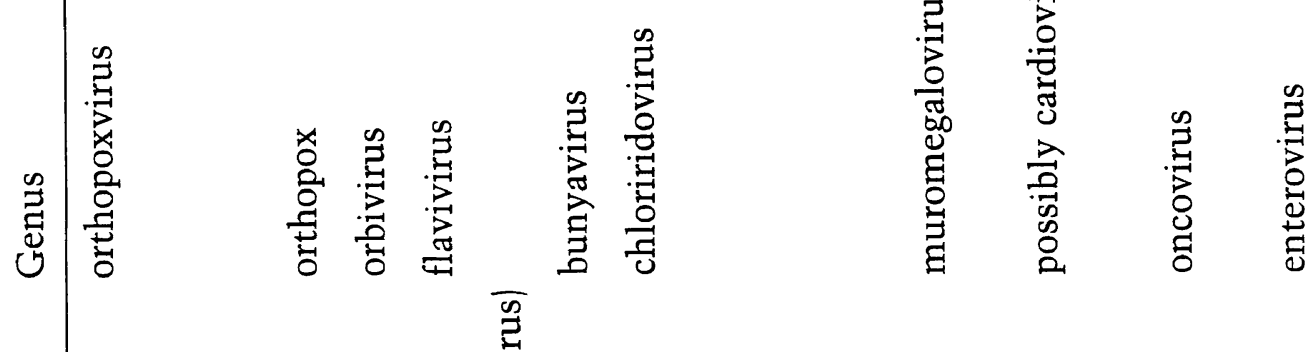

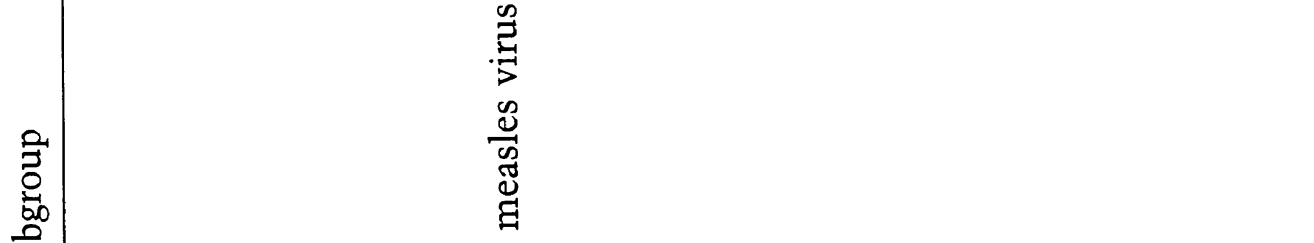

U

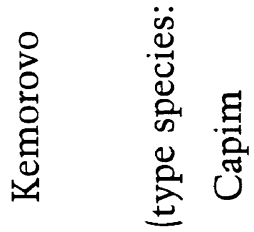

๑ุ

泀

苞

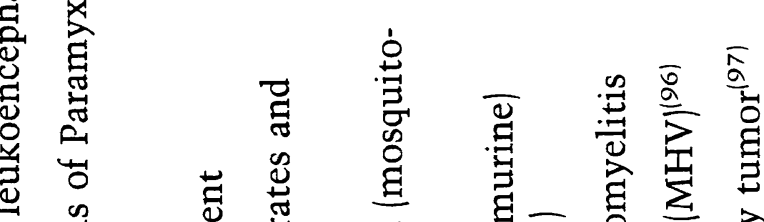

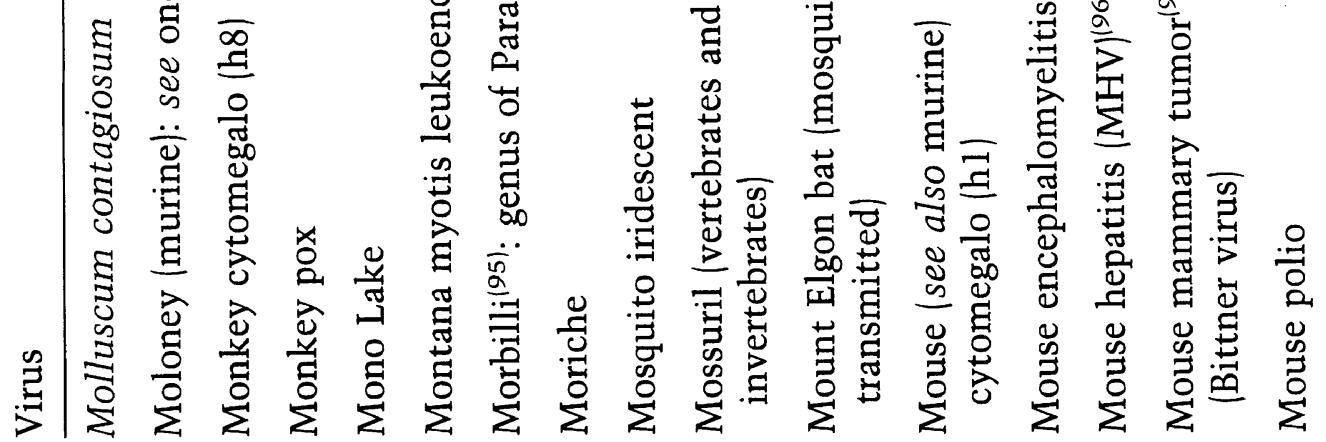



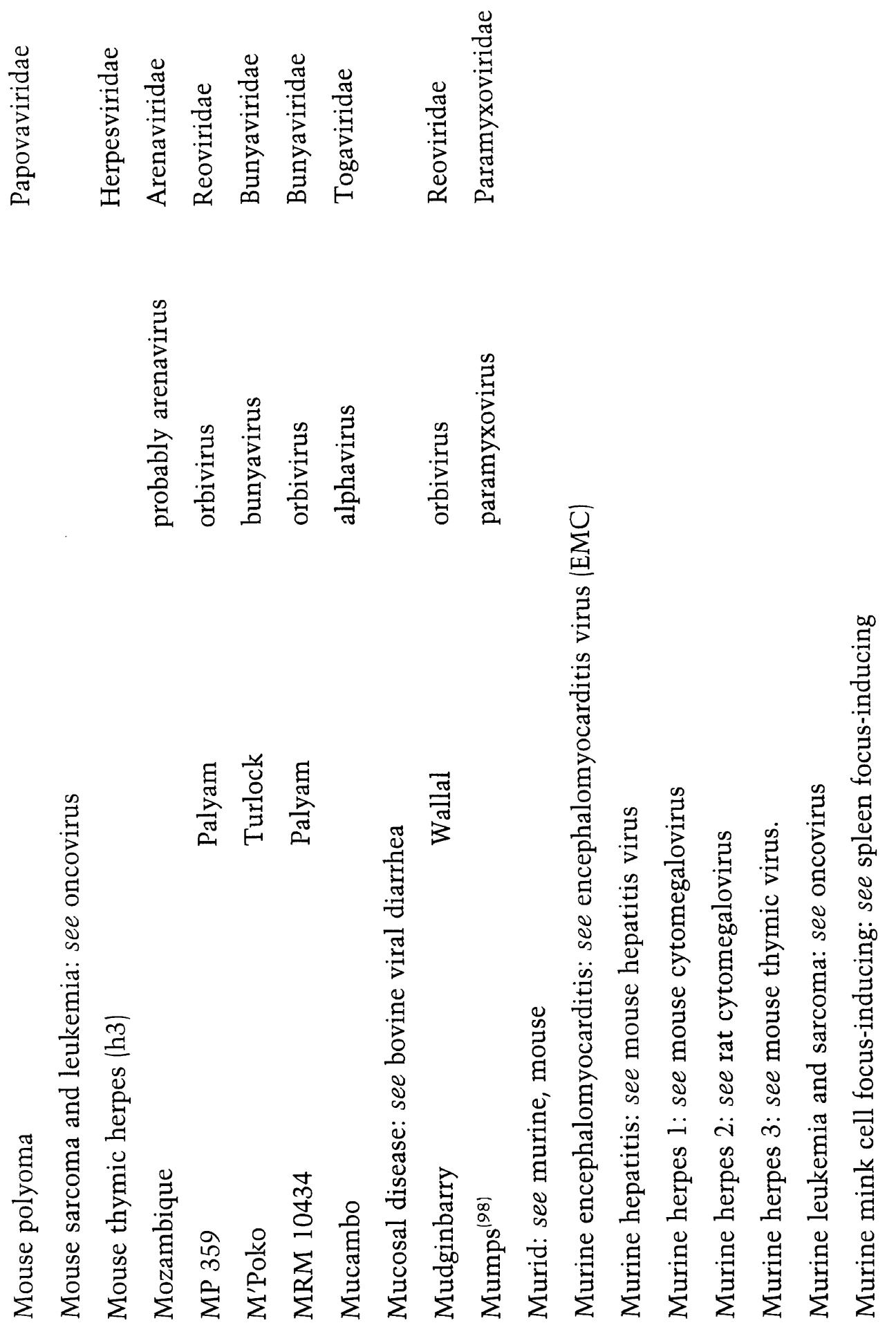


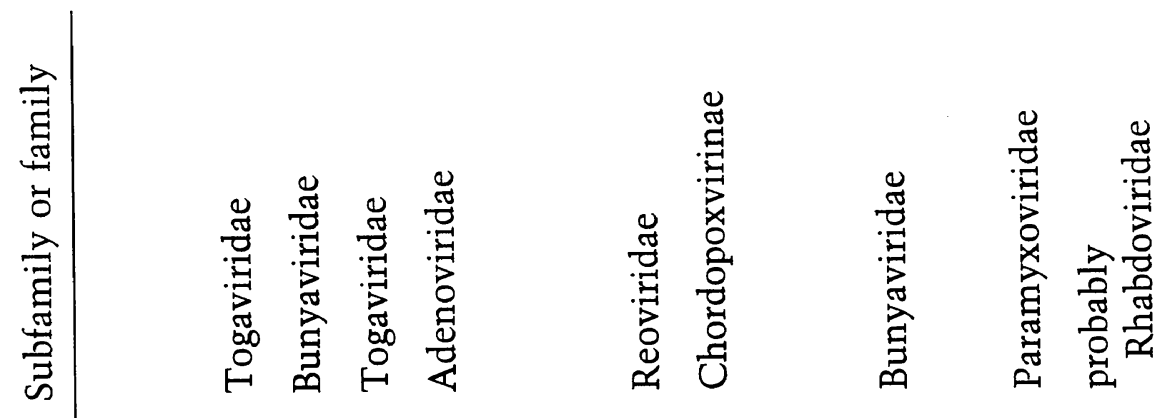

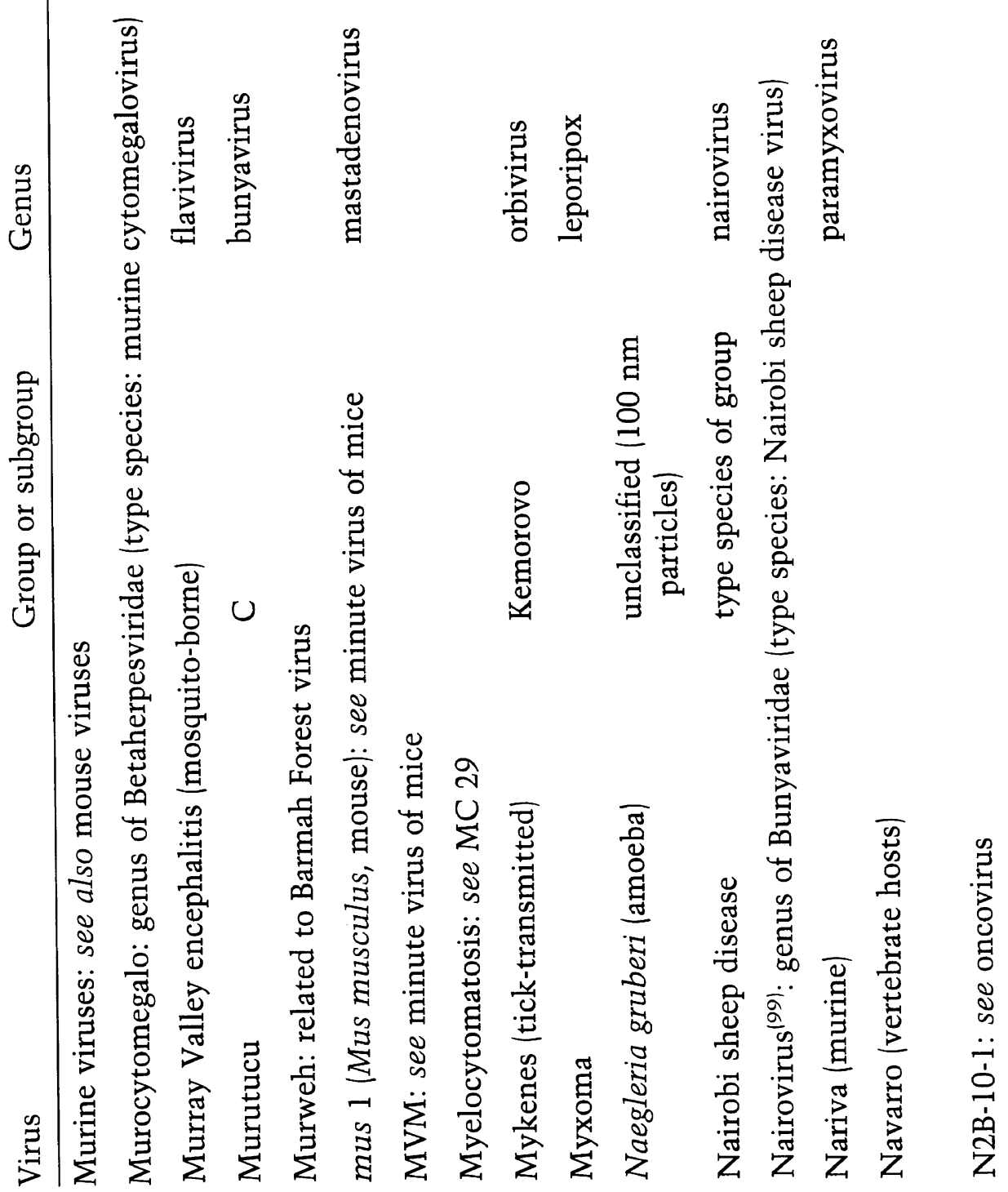



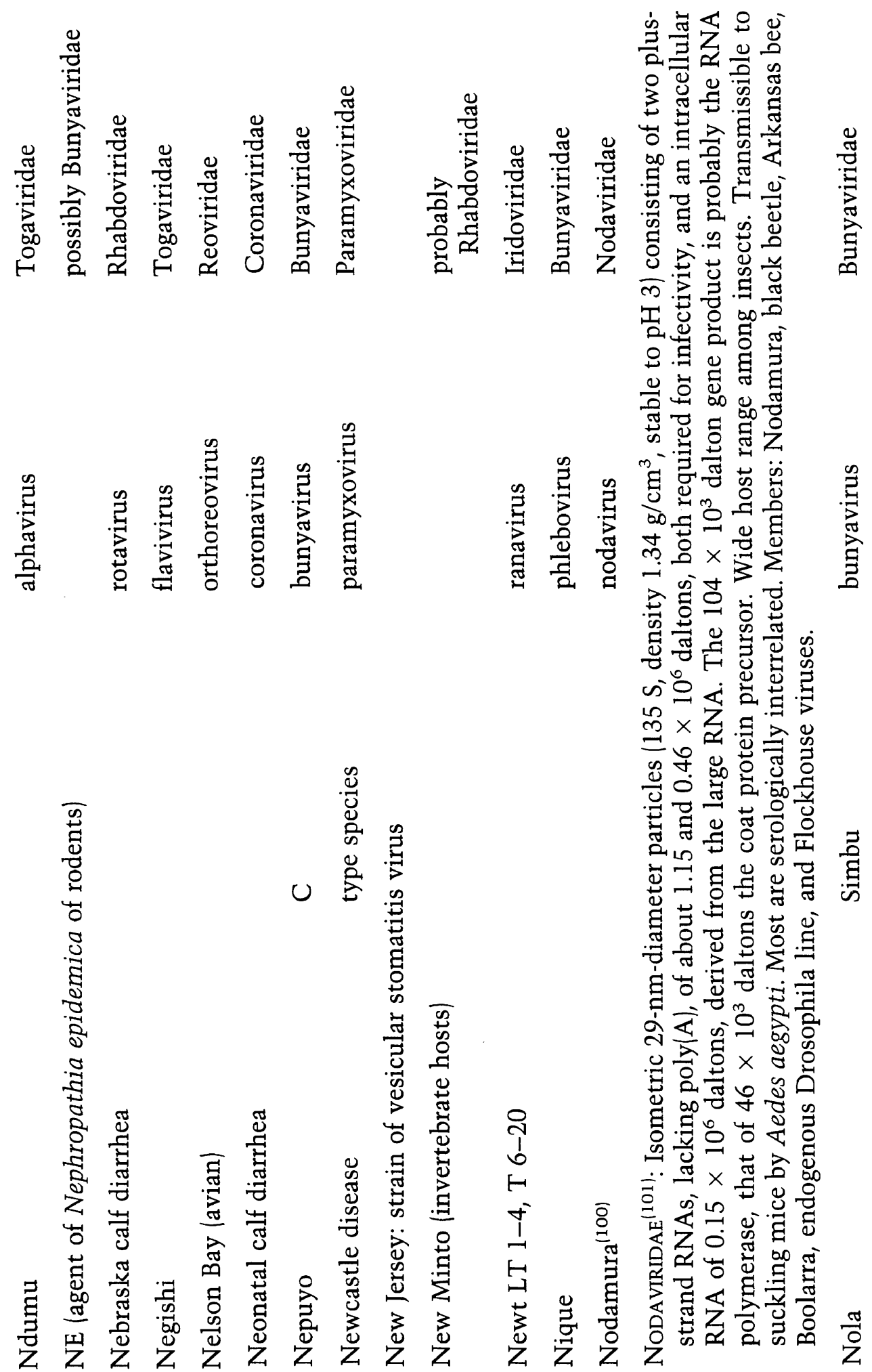


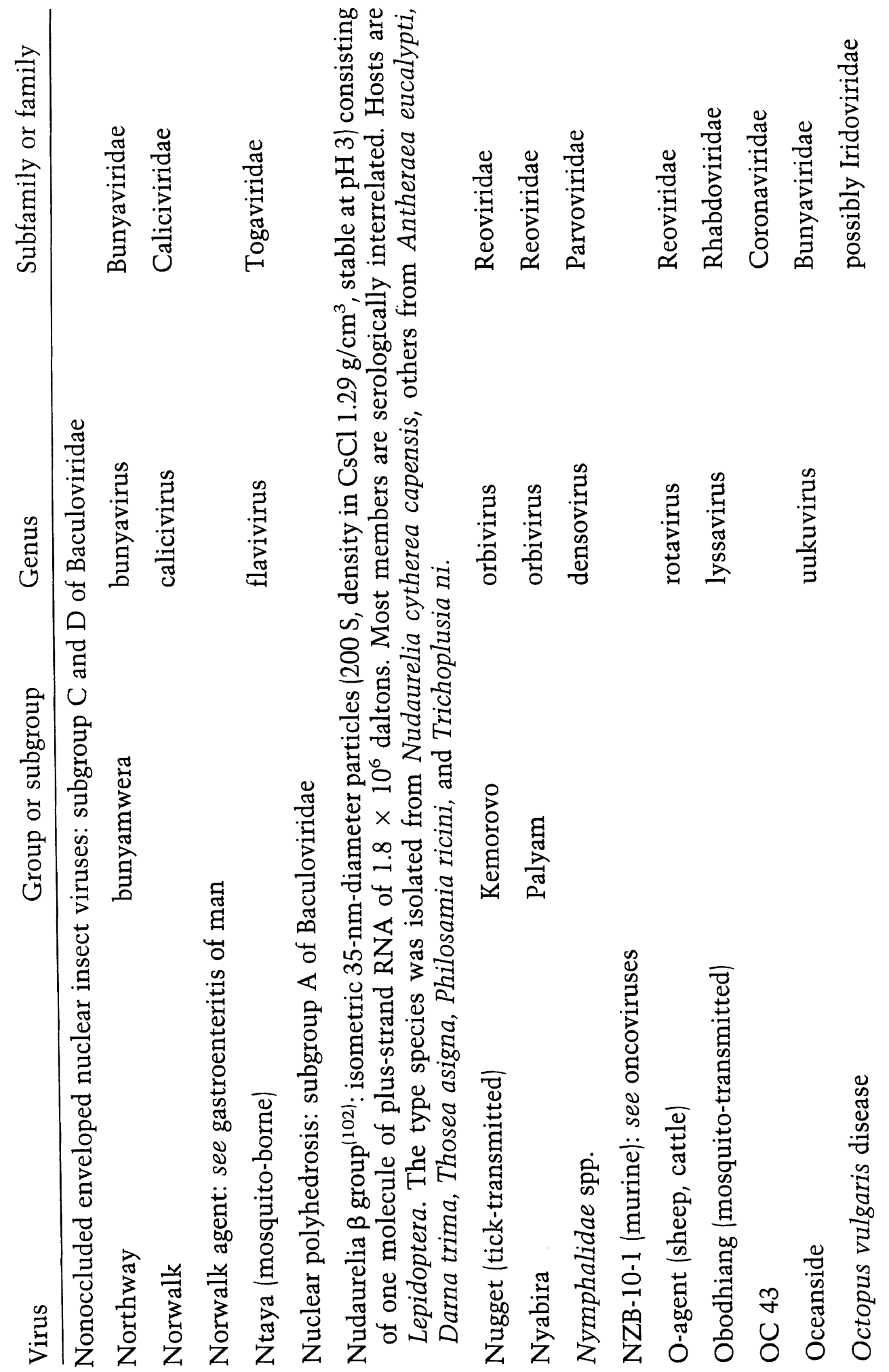



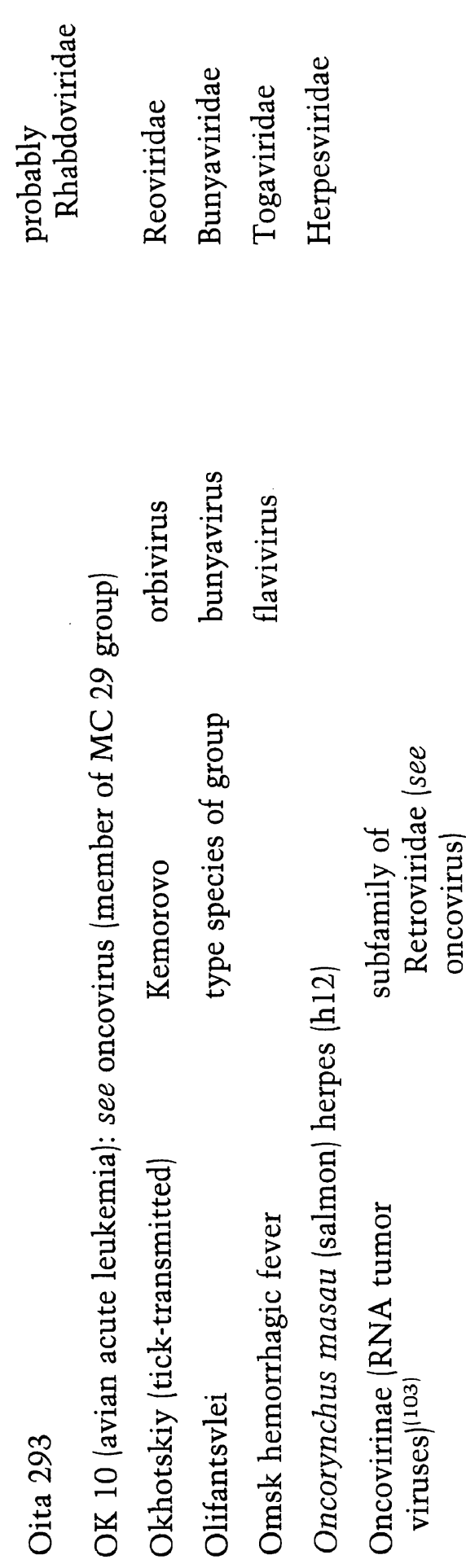

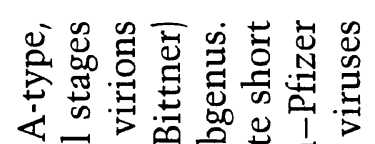

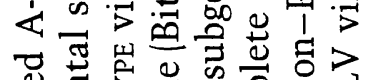
通

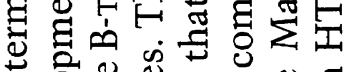

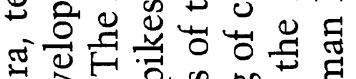
원 50

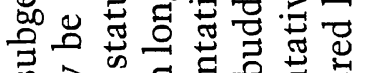
के

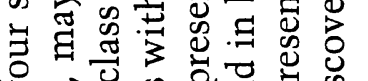

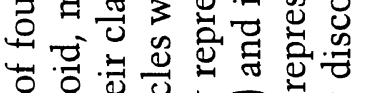

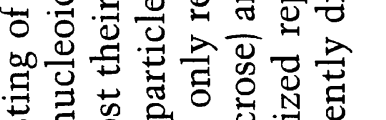

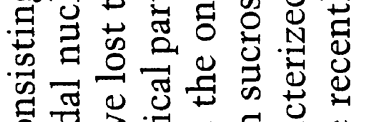
5 항

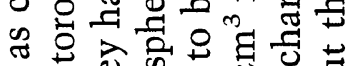

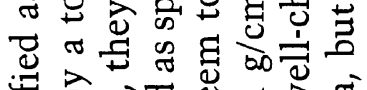

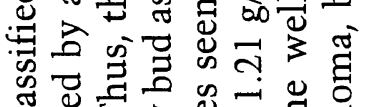

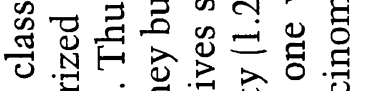

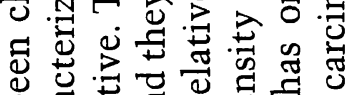

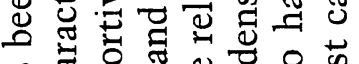

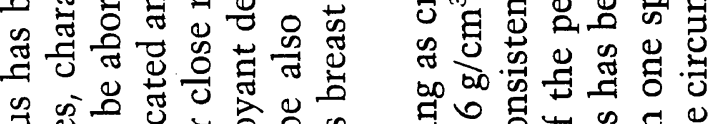

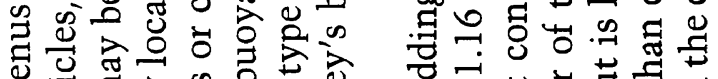

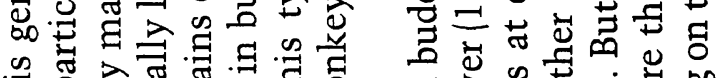

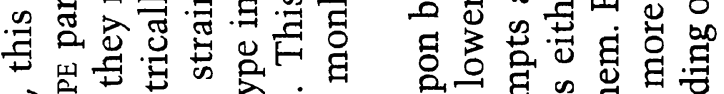

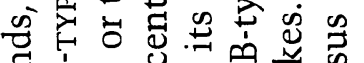

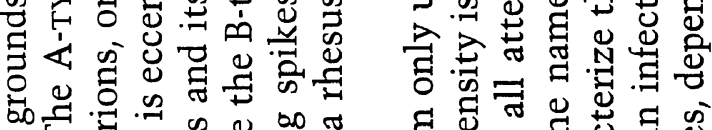

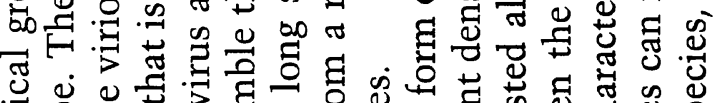

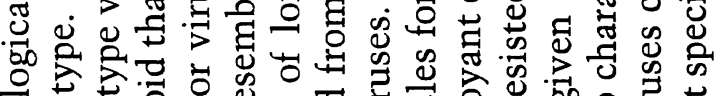

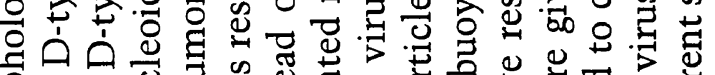

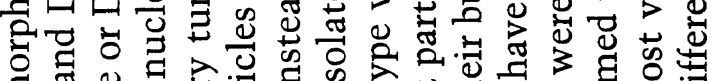

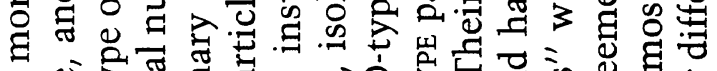

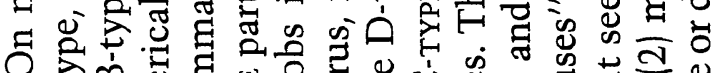

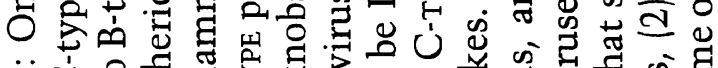

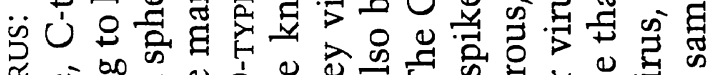

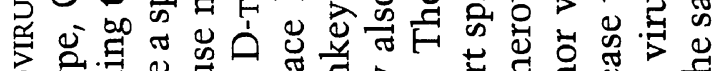

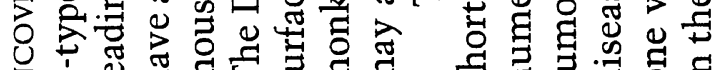

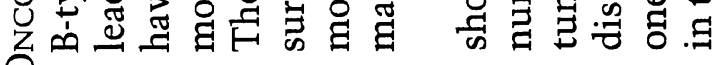




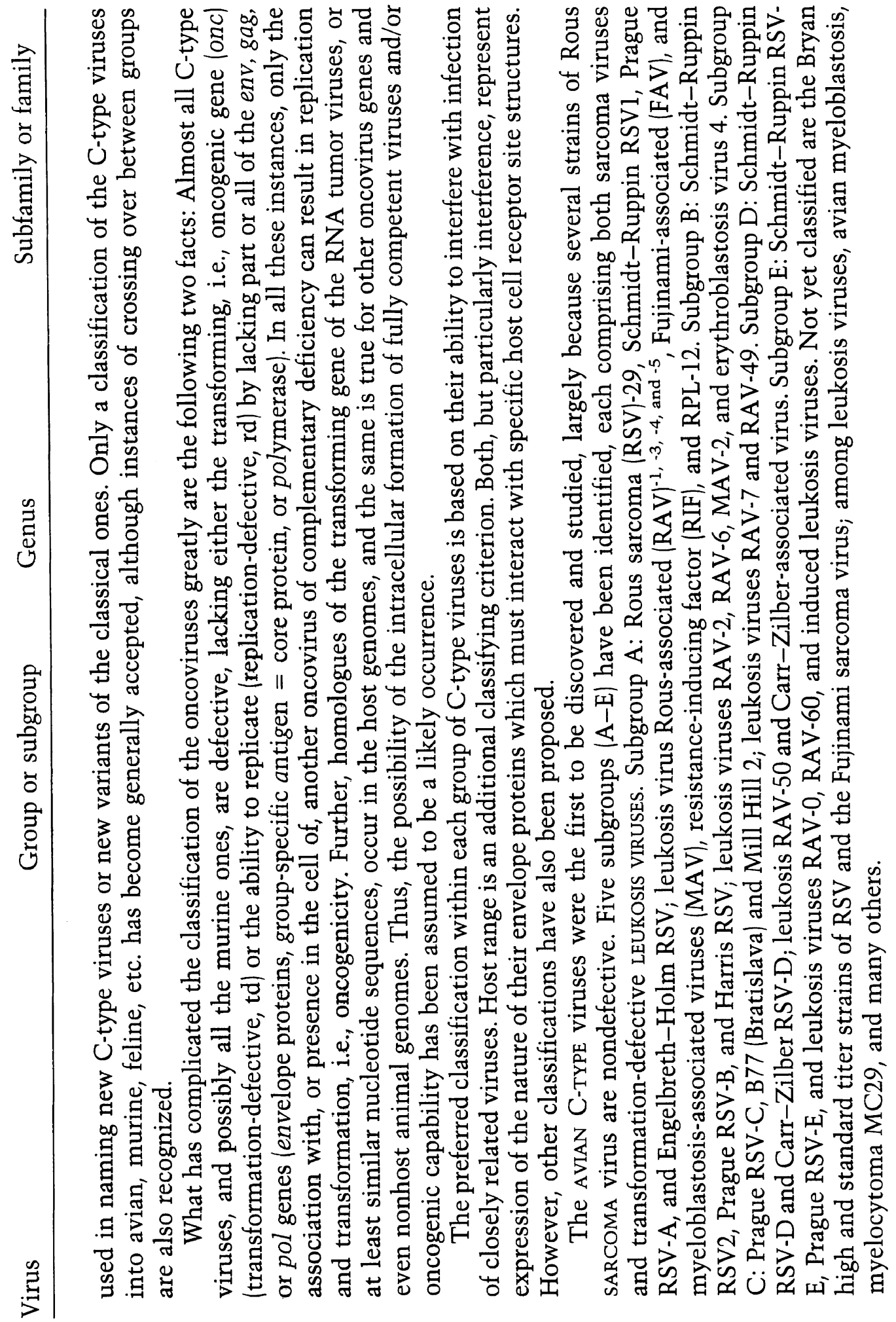




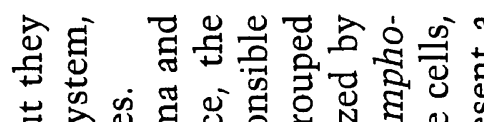

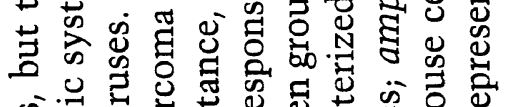

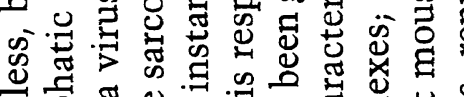

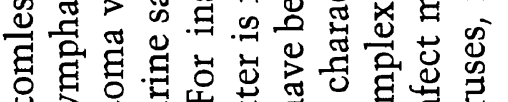
茗至 总点

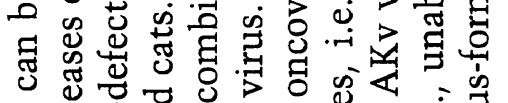

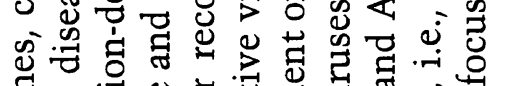

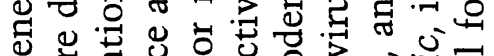

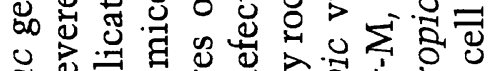

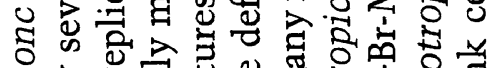

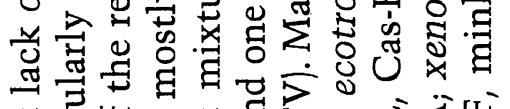

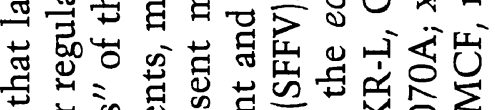
क क

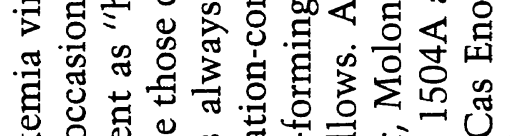

岳离芯 象泀 韋㤎 ปิ \&

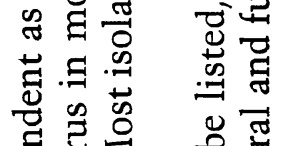

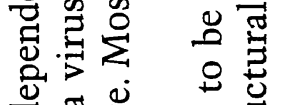

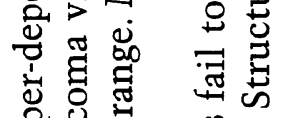

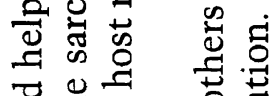

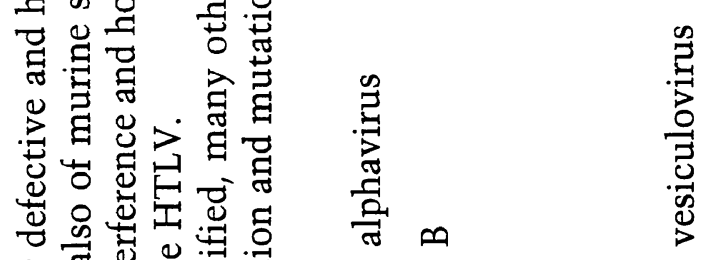

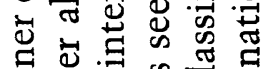

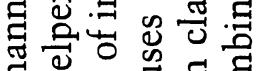
घ․ㅗ. \& ⿷匚

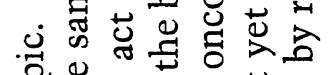
㐘 ఏ

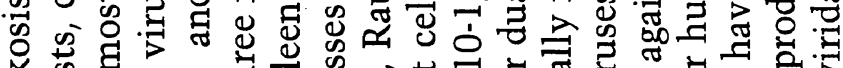

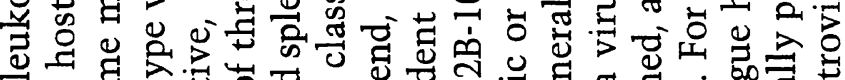

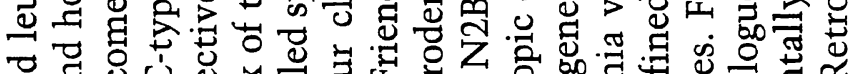

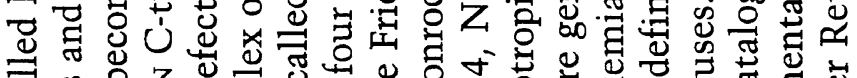

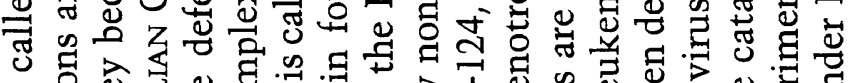

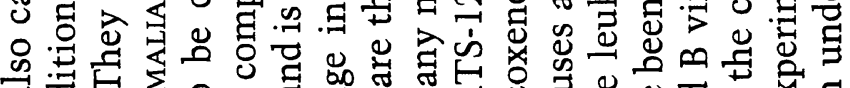
कै क О

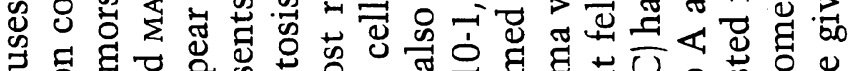

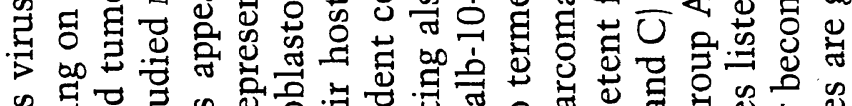

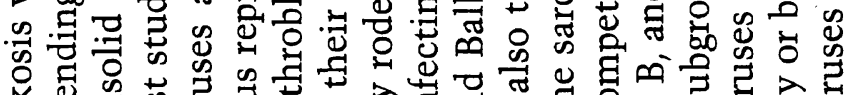

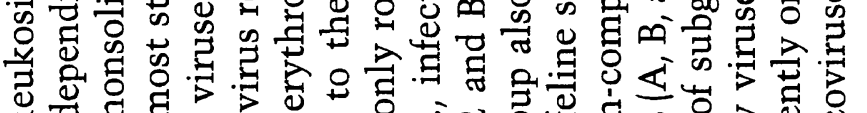

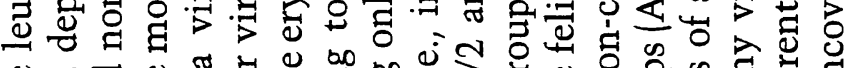
\&

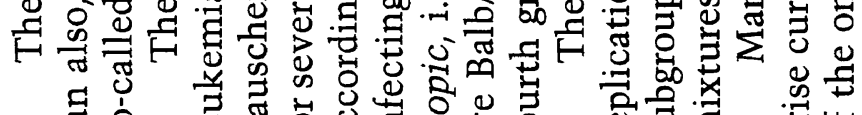

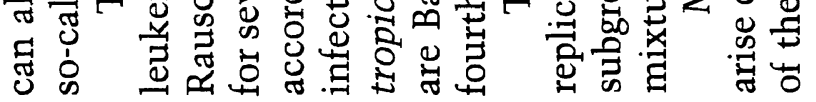

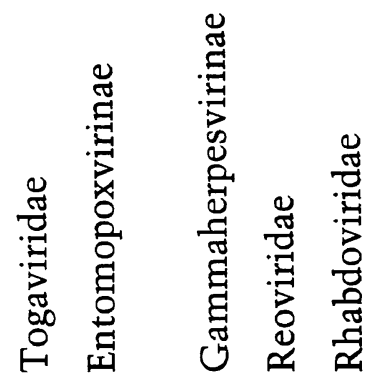

奈

$\mathscr{F}_{0}$

○్

$\stackrel{0}{3}$

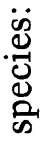

空

\&

苟

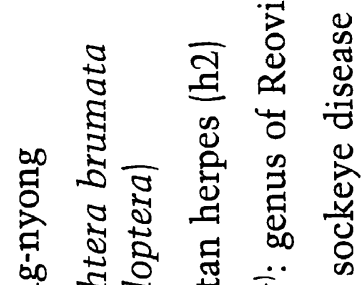

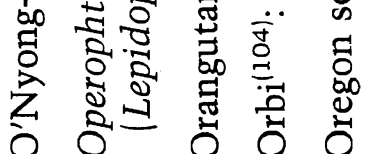




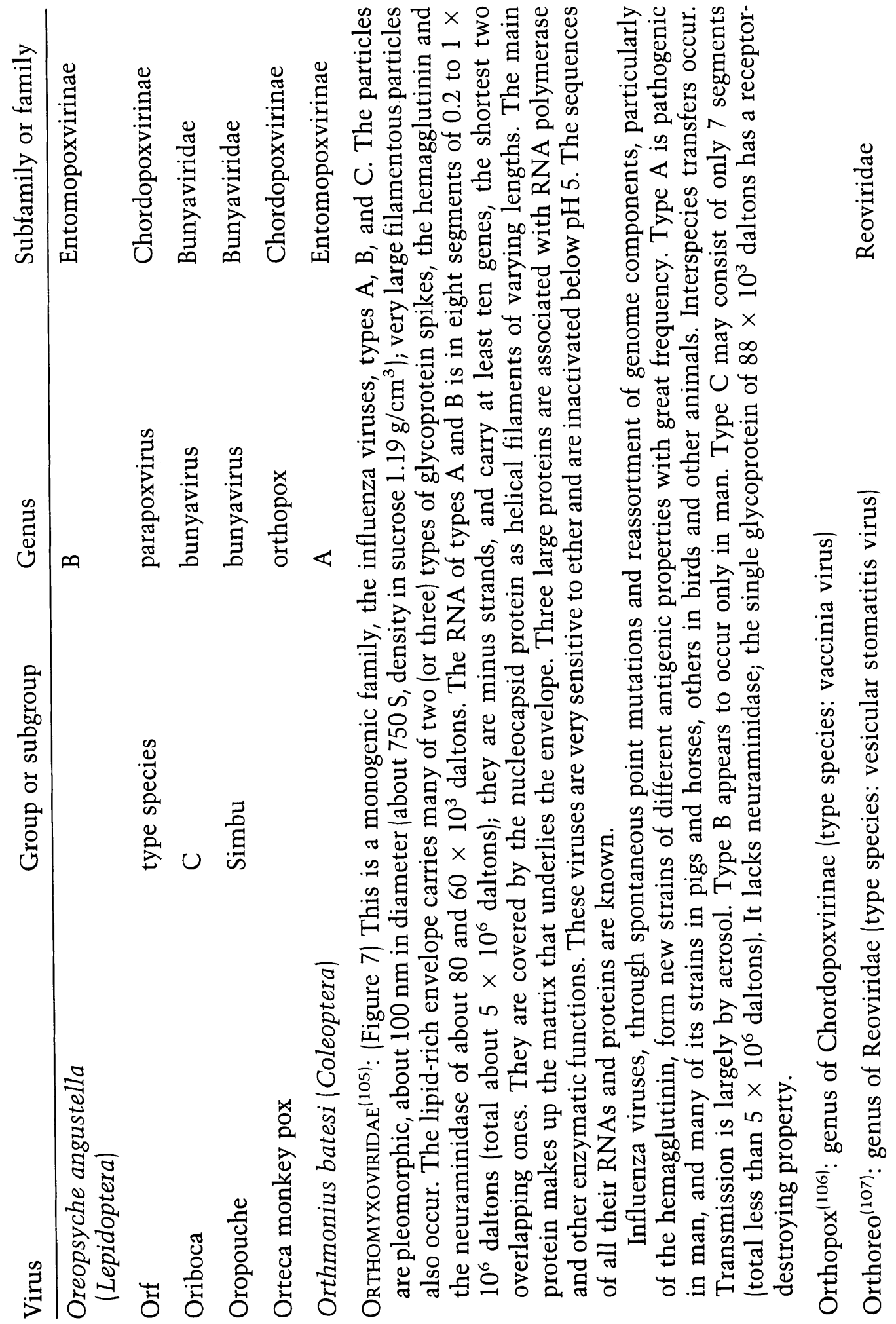



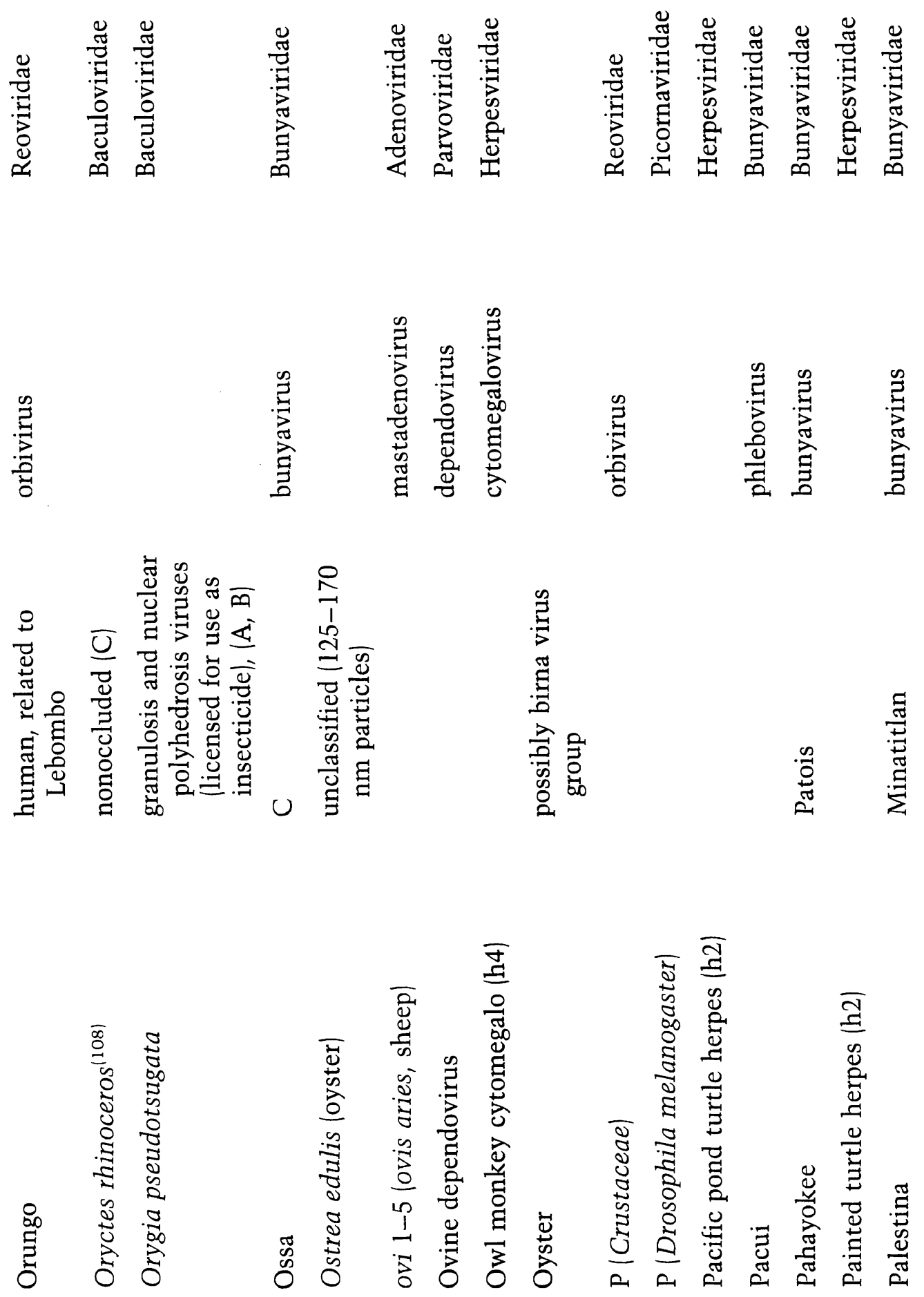


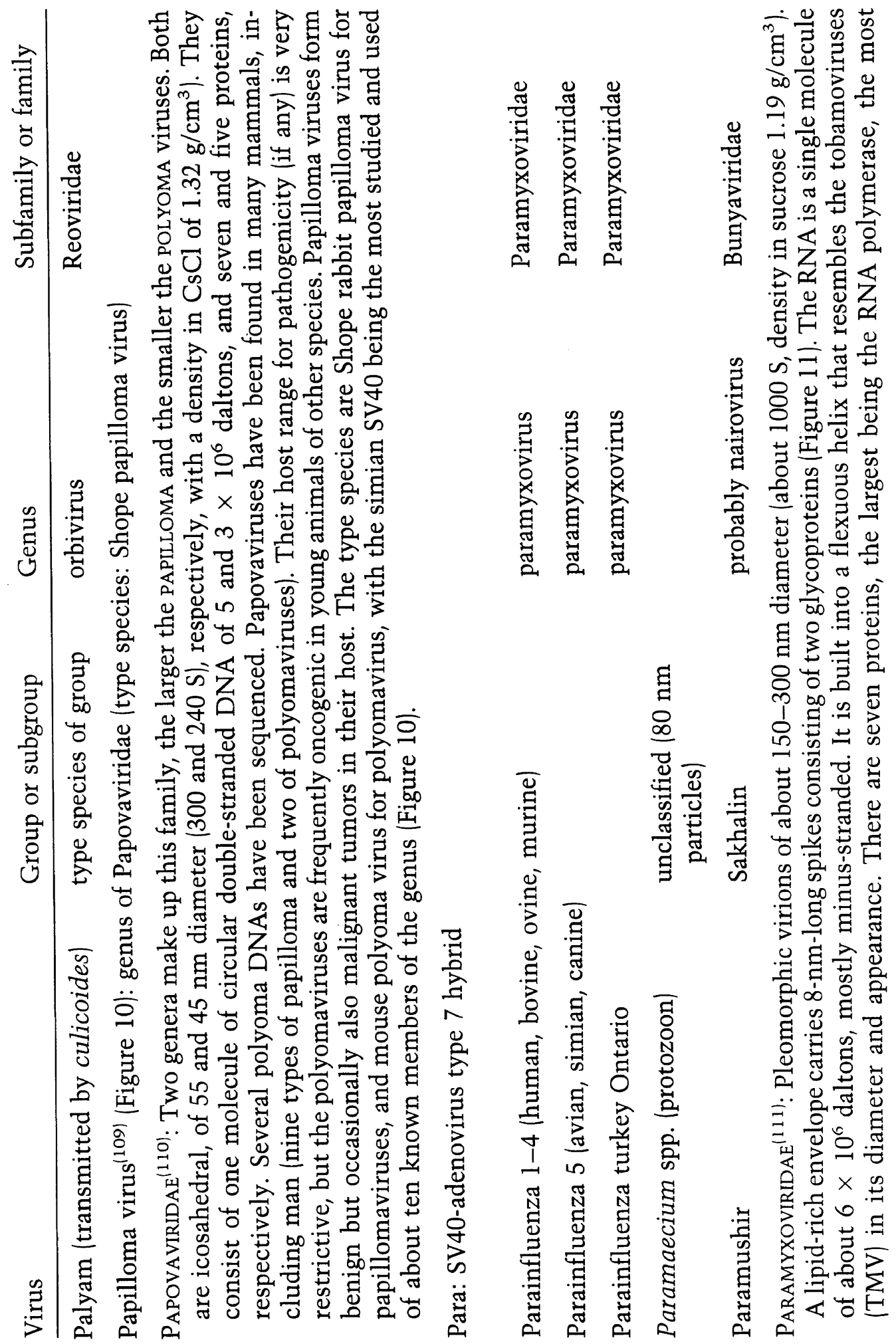




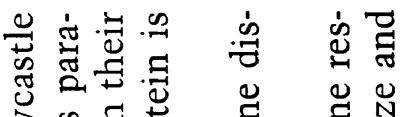

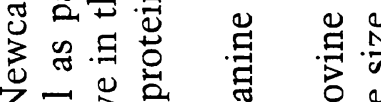

Z

声交究 吾

$3 \approx \mathbb{S}$

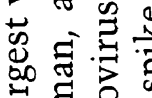

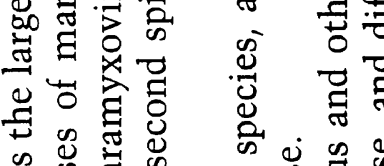

胥

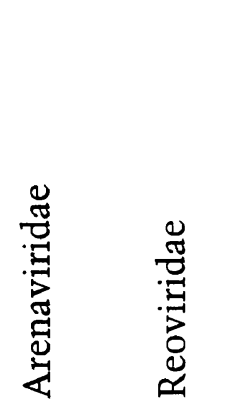

$\rightarrow \infty \begin{gathered}0 \\ \infty\end{gathered}$

के में

胥点

苛

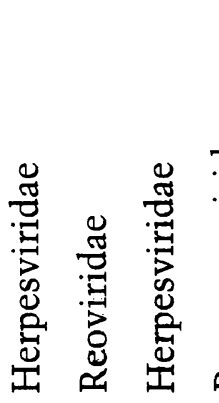

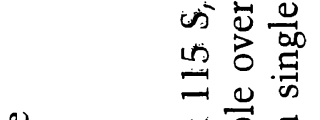

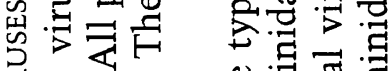

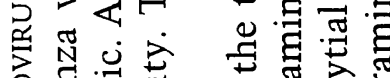

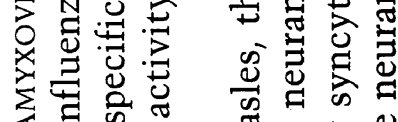

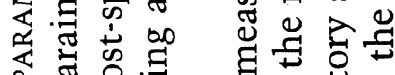

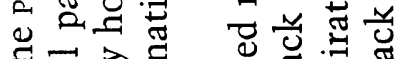

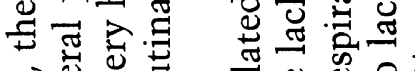

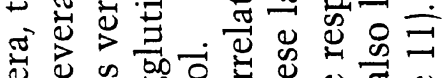

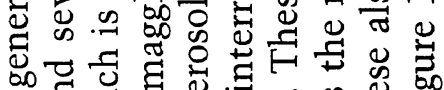

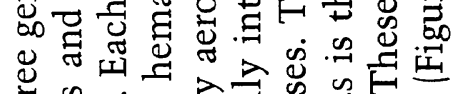

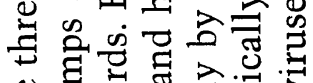

๑

플

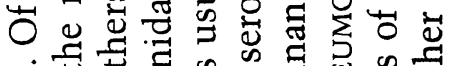

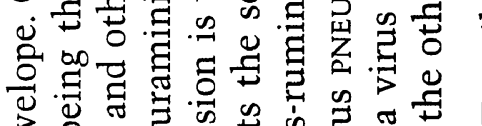

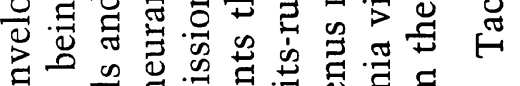

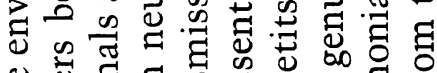

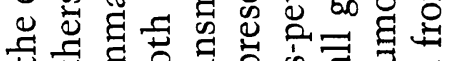

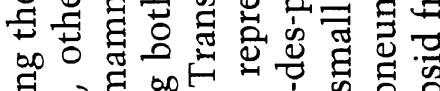

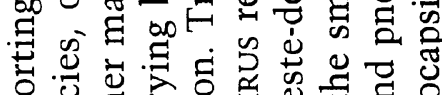

을

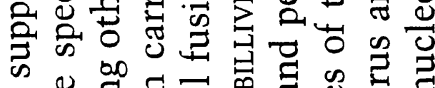

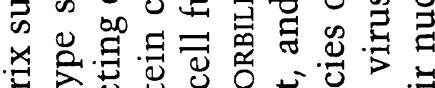

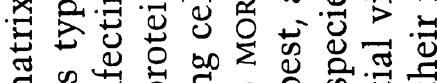

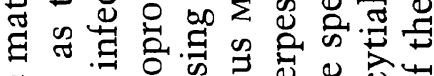

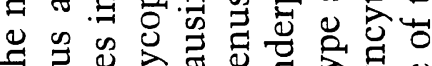

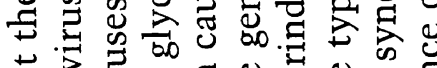

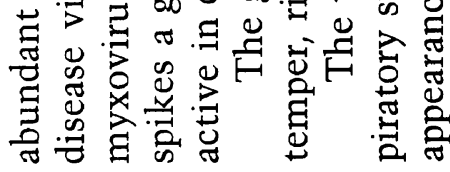

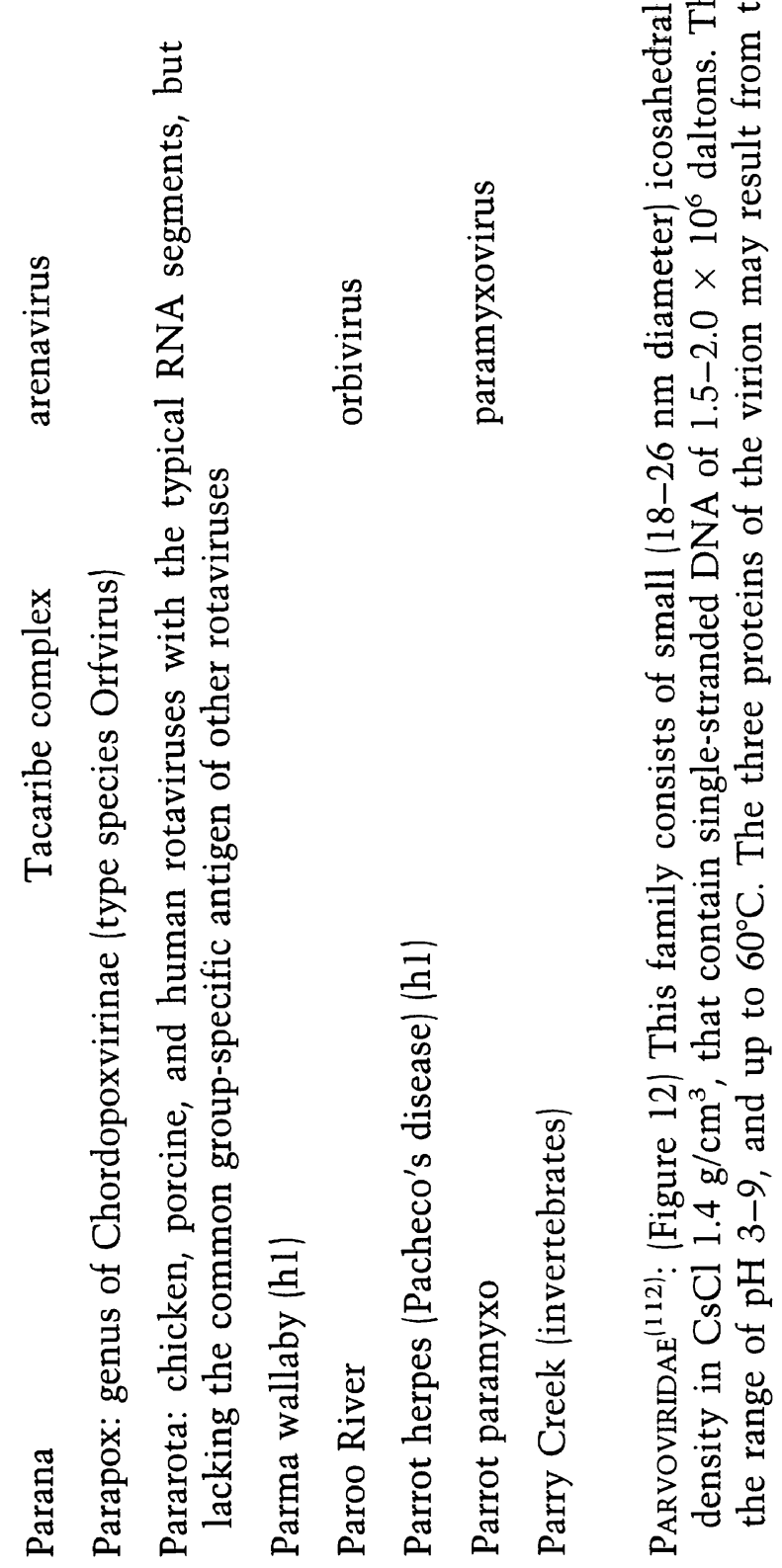




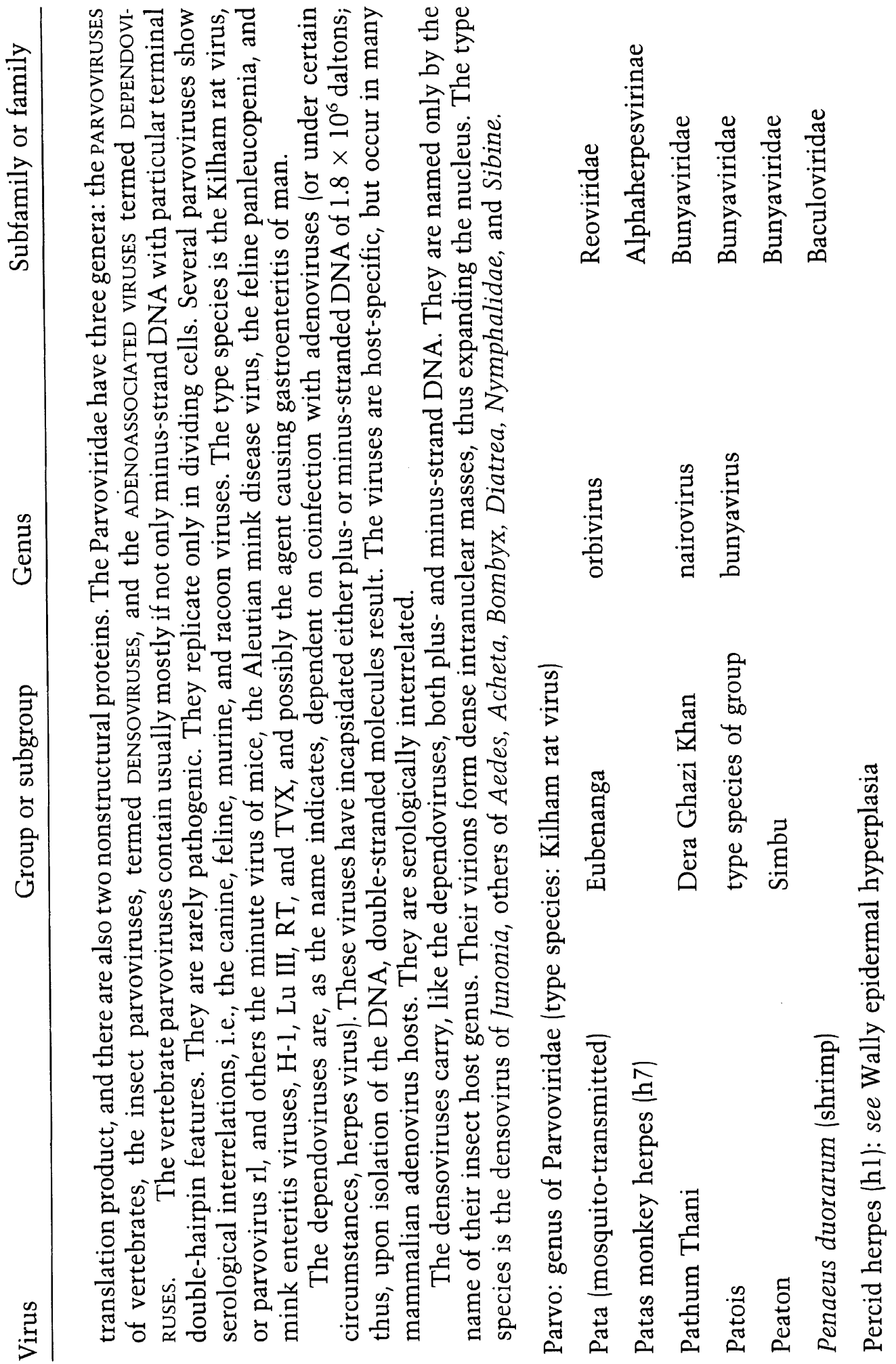



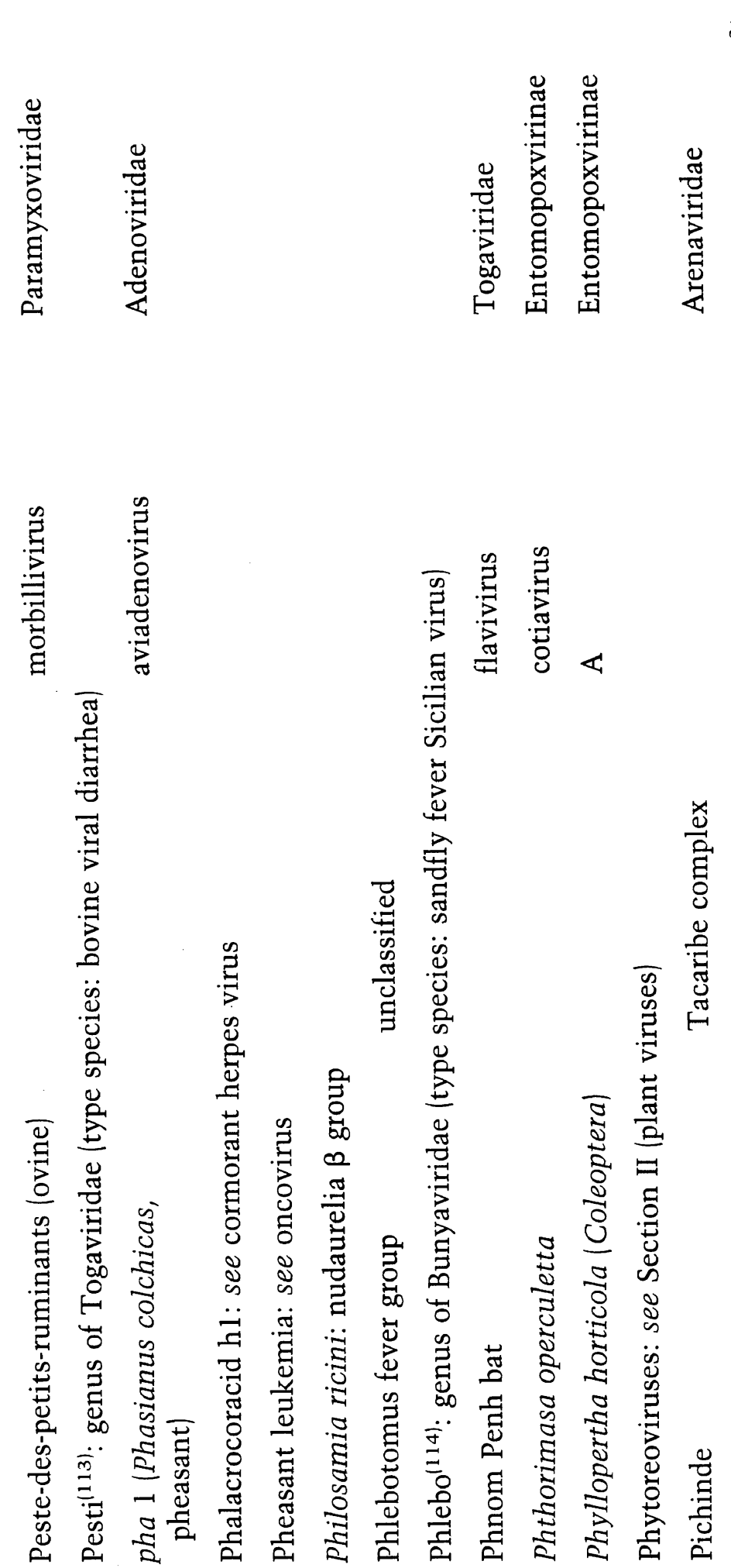

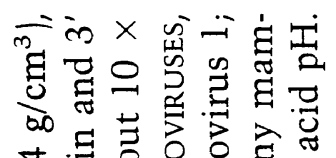

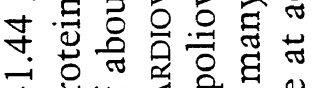

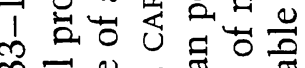
అ ర

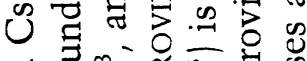
퓌 公 $\times z_{4} \frac{0}{0}$

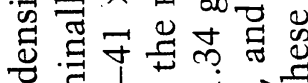

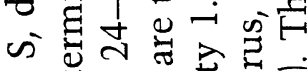
जि in \&

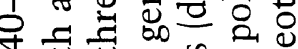
二苾声出出总 む)

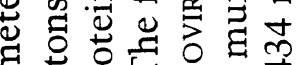

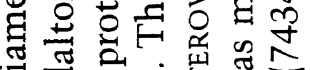

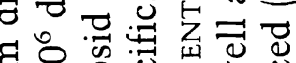
西으웛

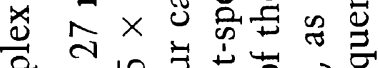

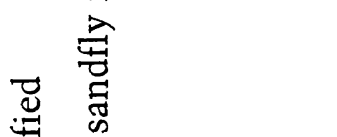

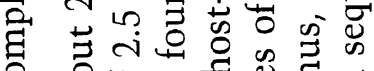

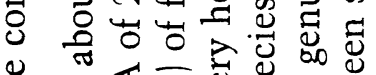
ㄴ $4 \overline{0}$ ชู \& ज .

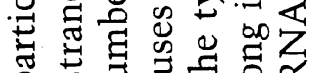

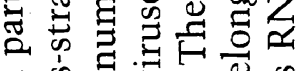

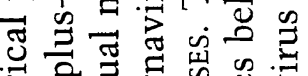

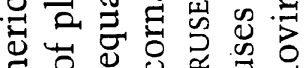

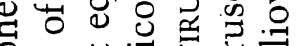

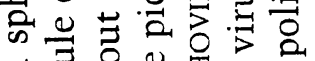

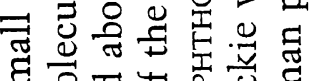

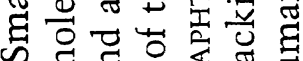

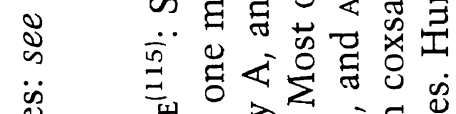

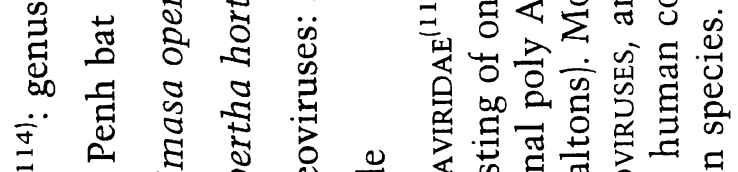

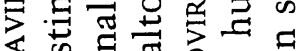

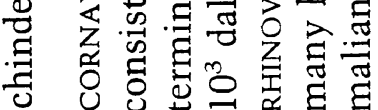

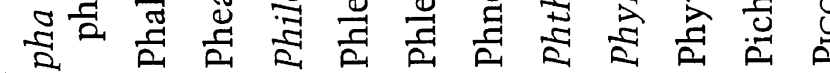




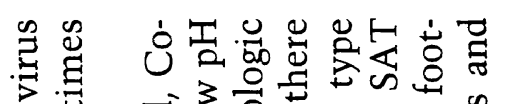

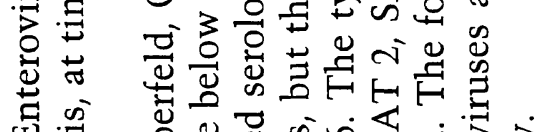

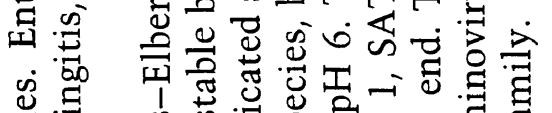

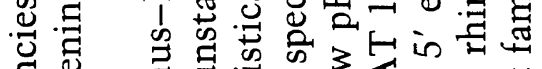

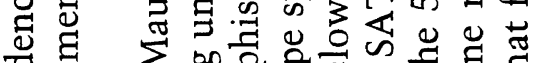

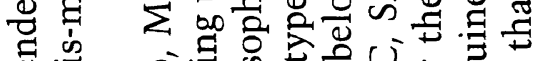
D 0.

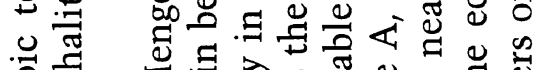

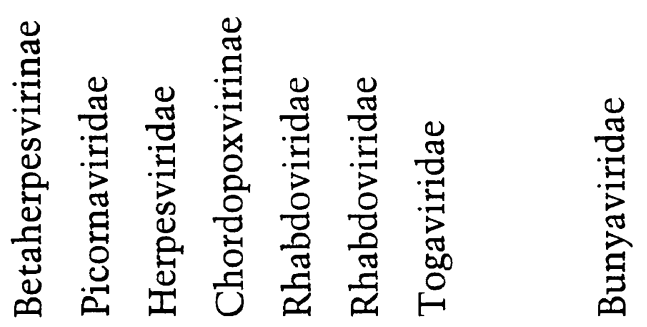

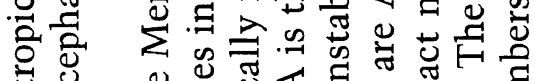

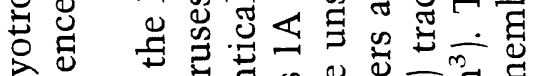

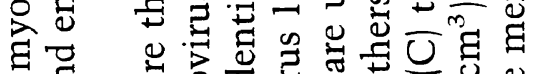

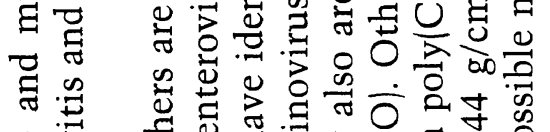

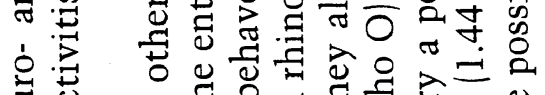

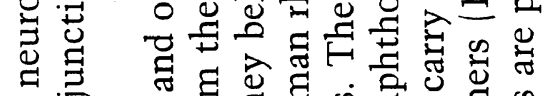

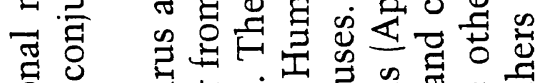

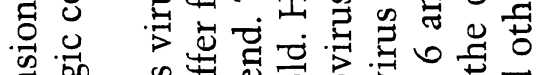

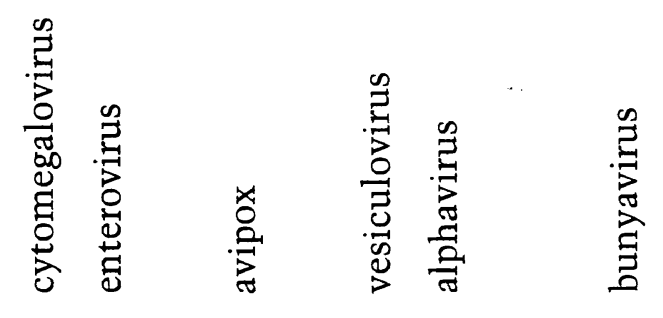

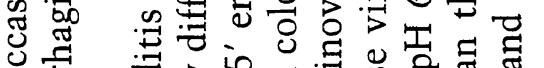

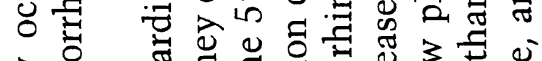

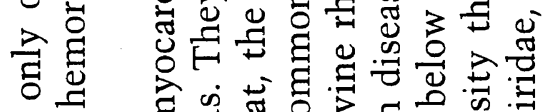

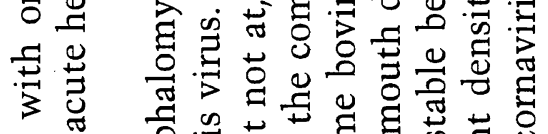

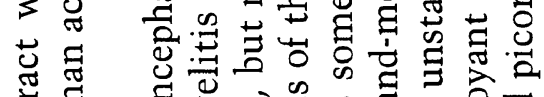

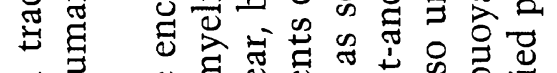

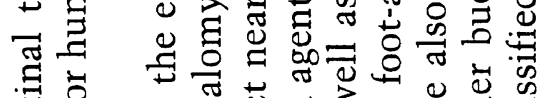

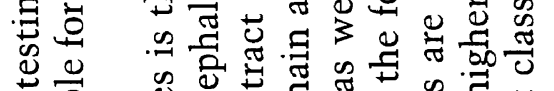

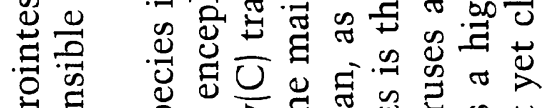

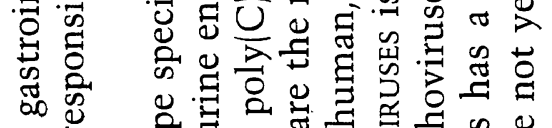

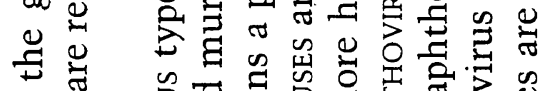

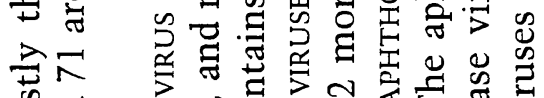
क्

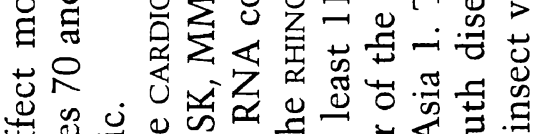

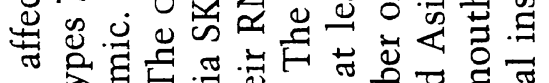
क人

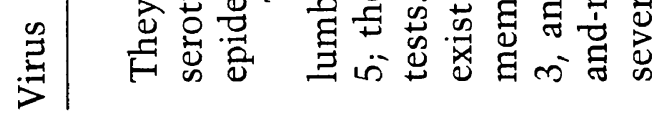

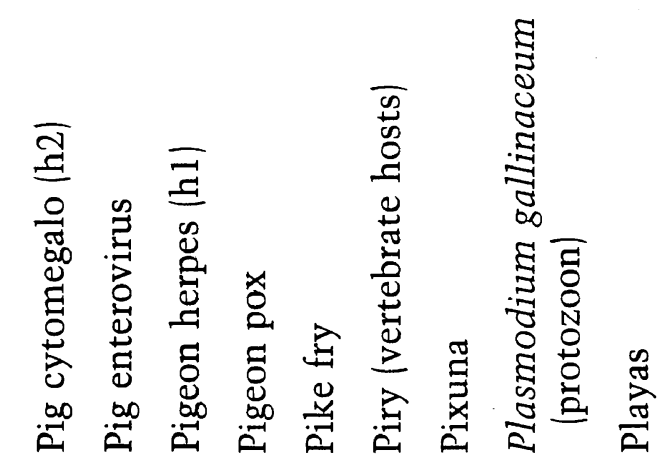



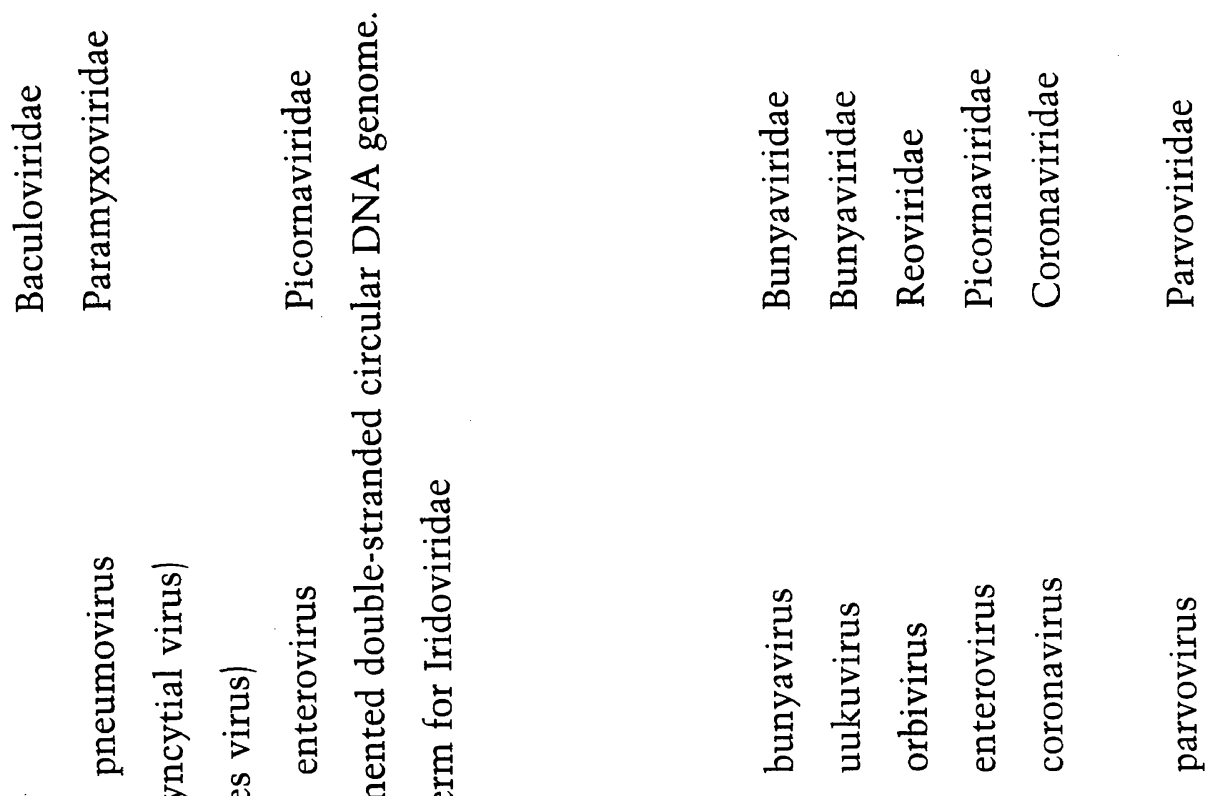

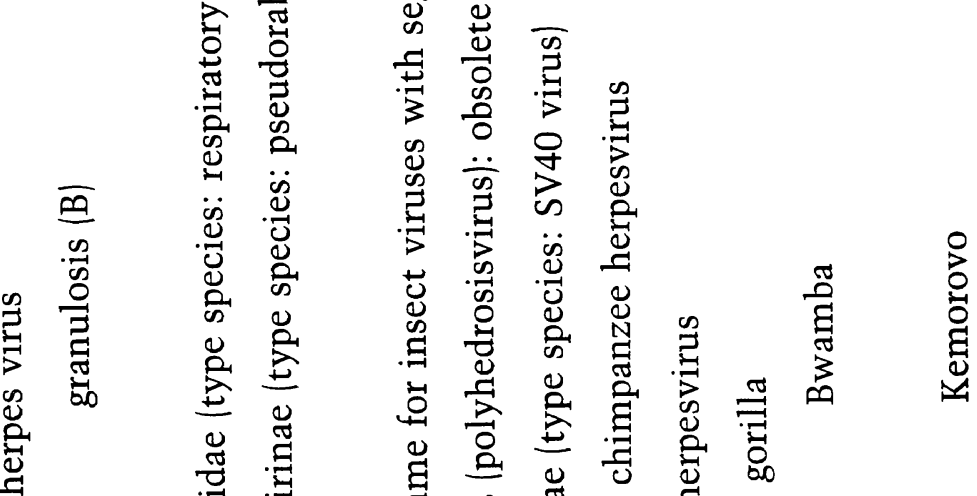

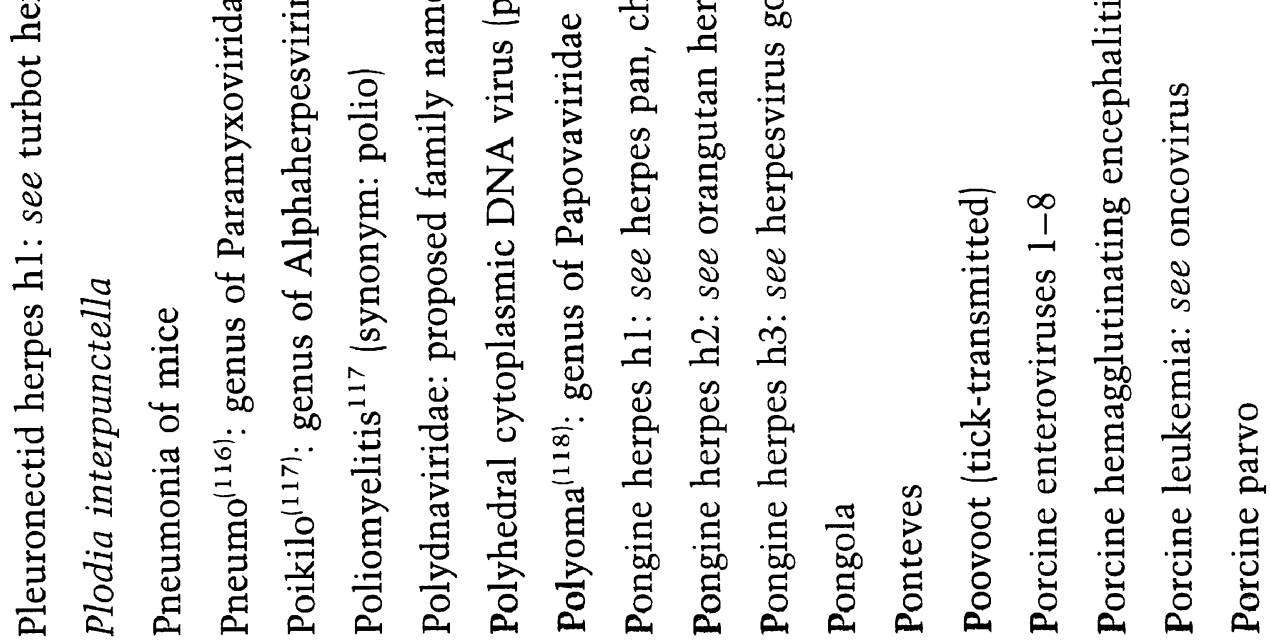




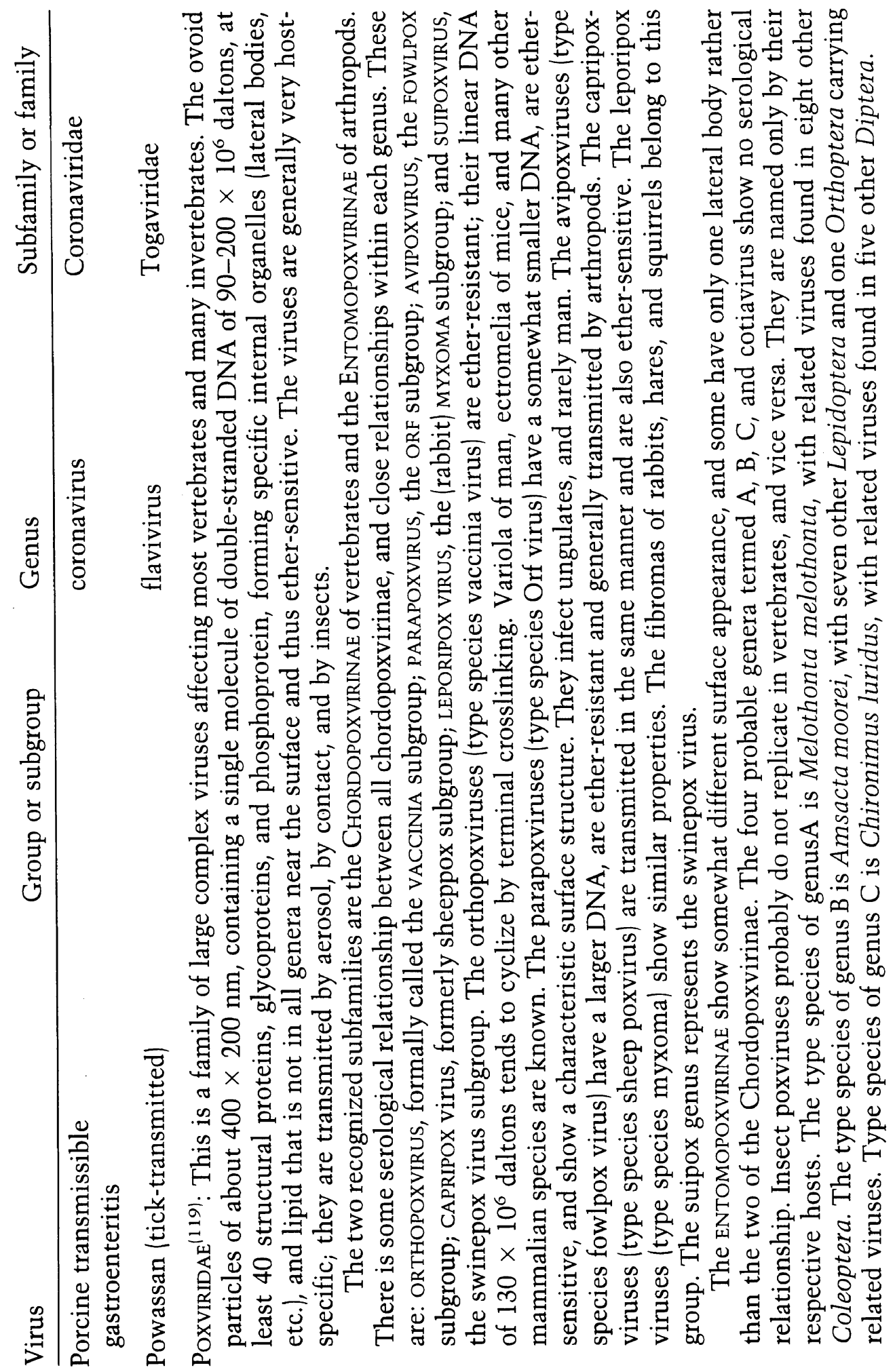



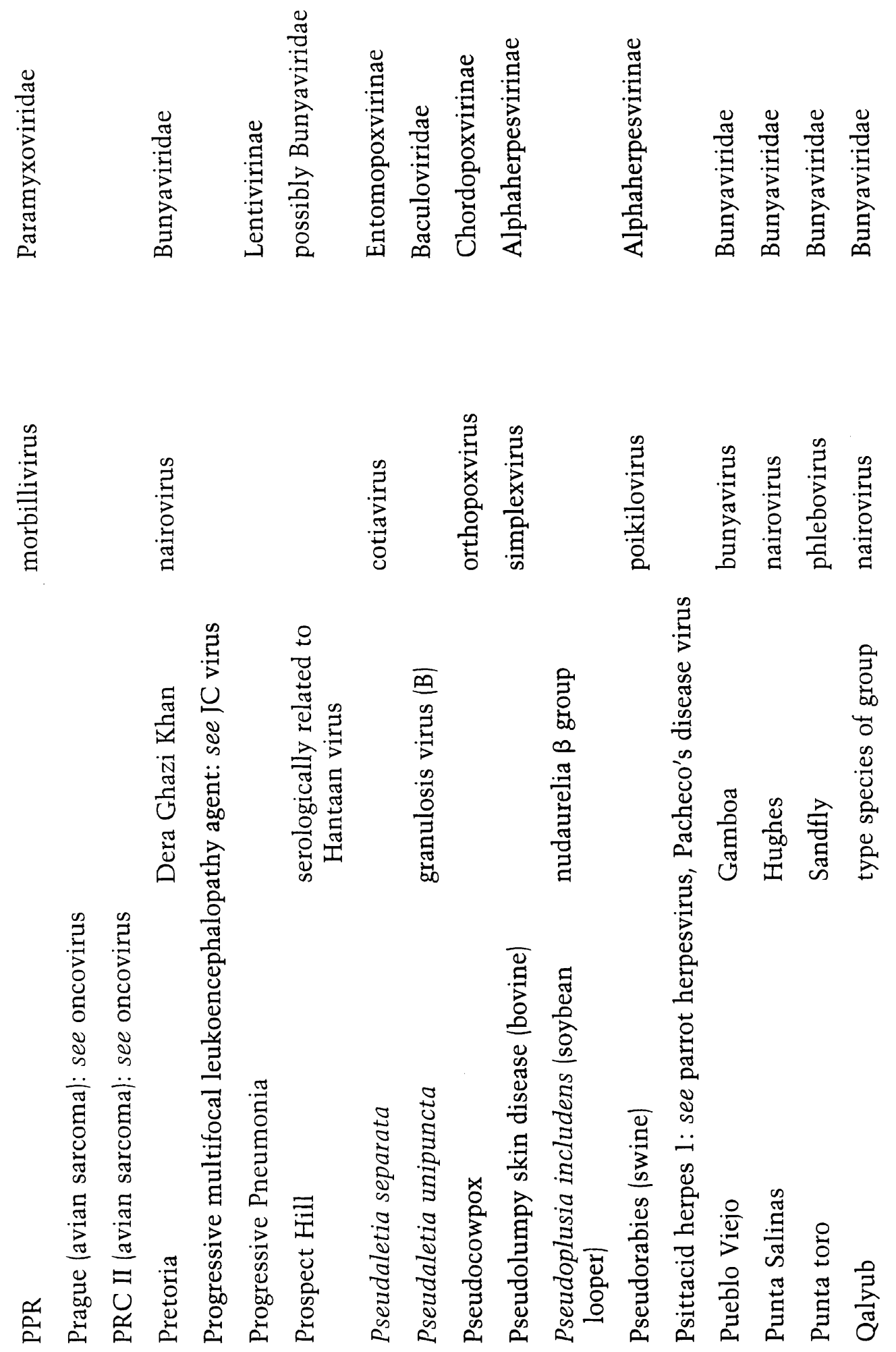


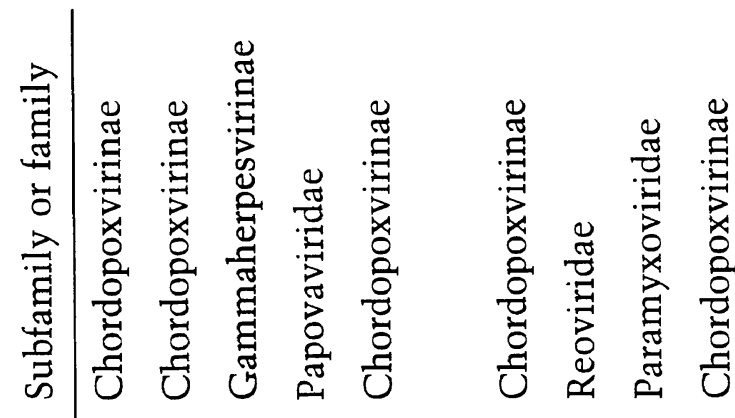

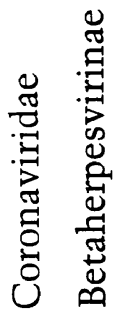

|

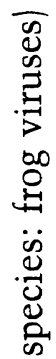

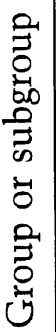

茵

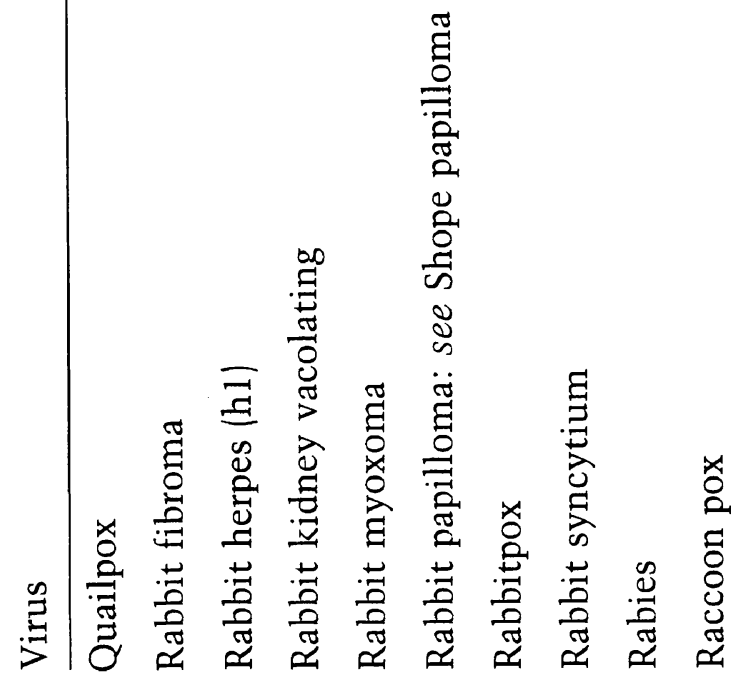

茫

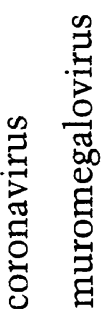
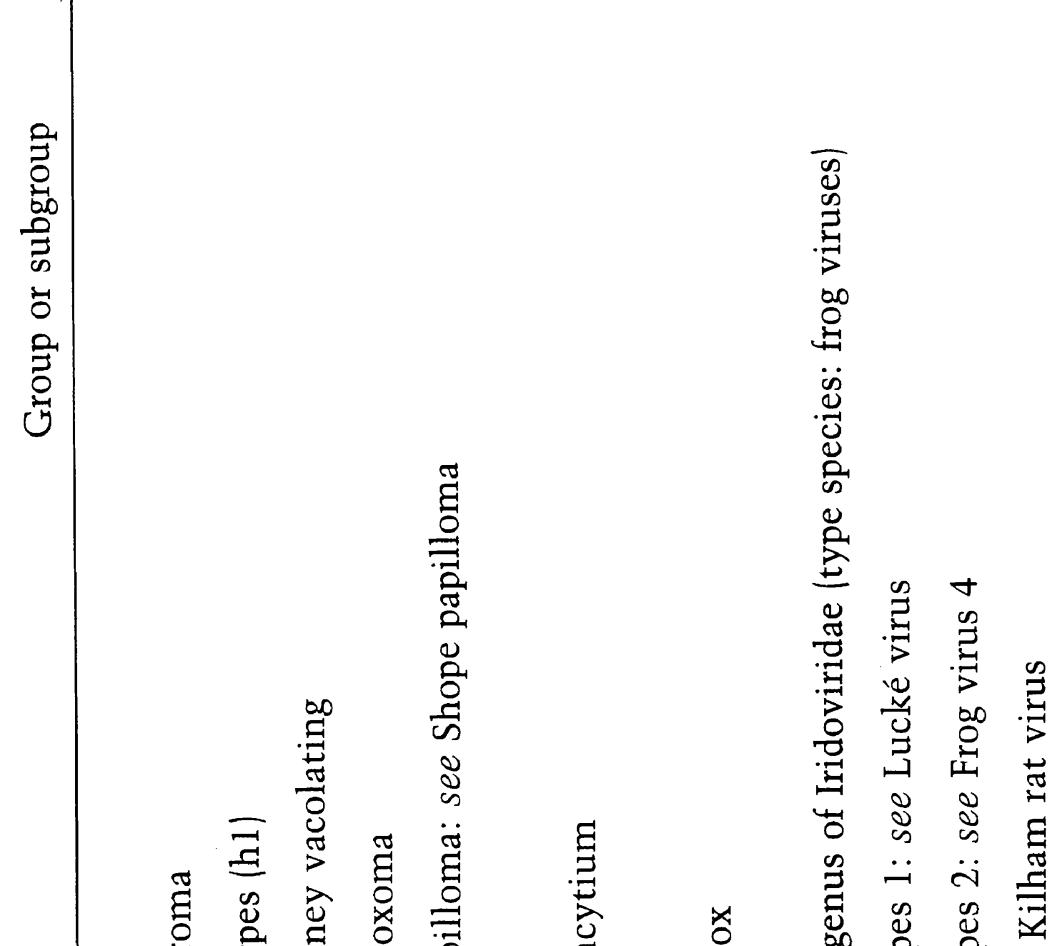

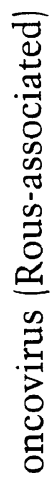

\&

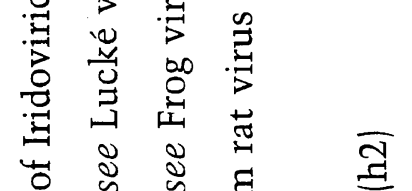

$\therefore 8$ 


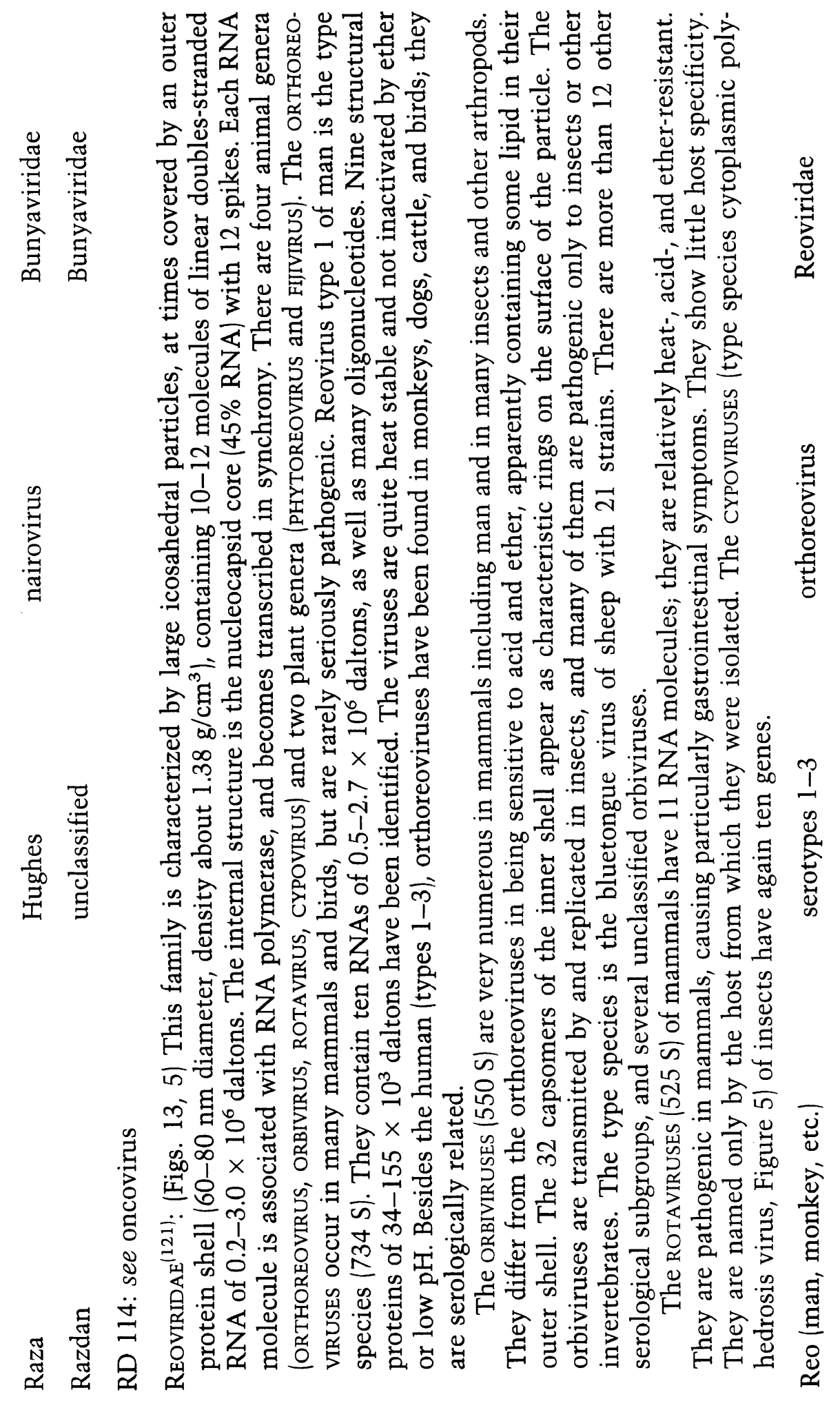


SECTION I

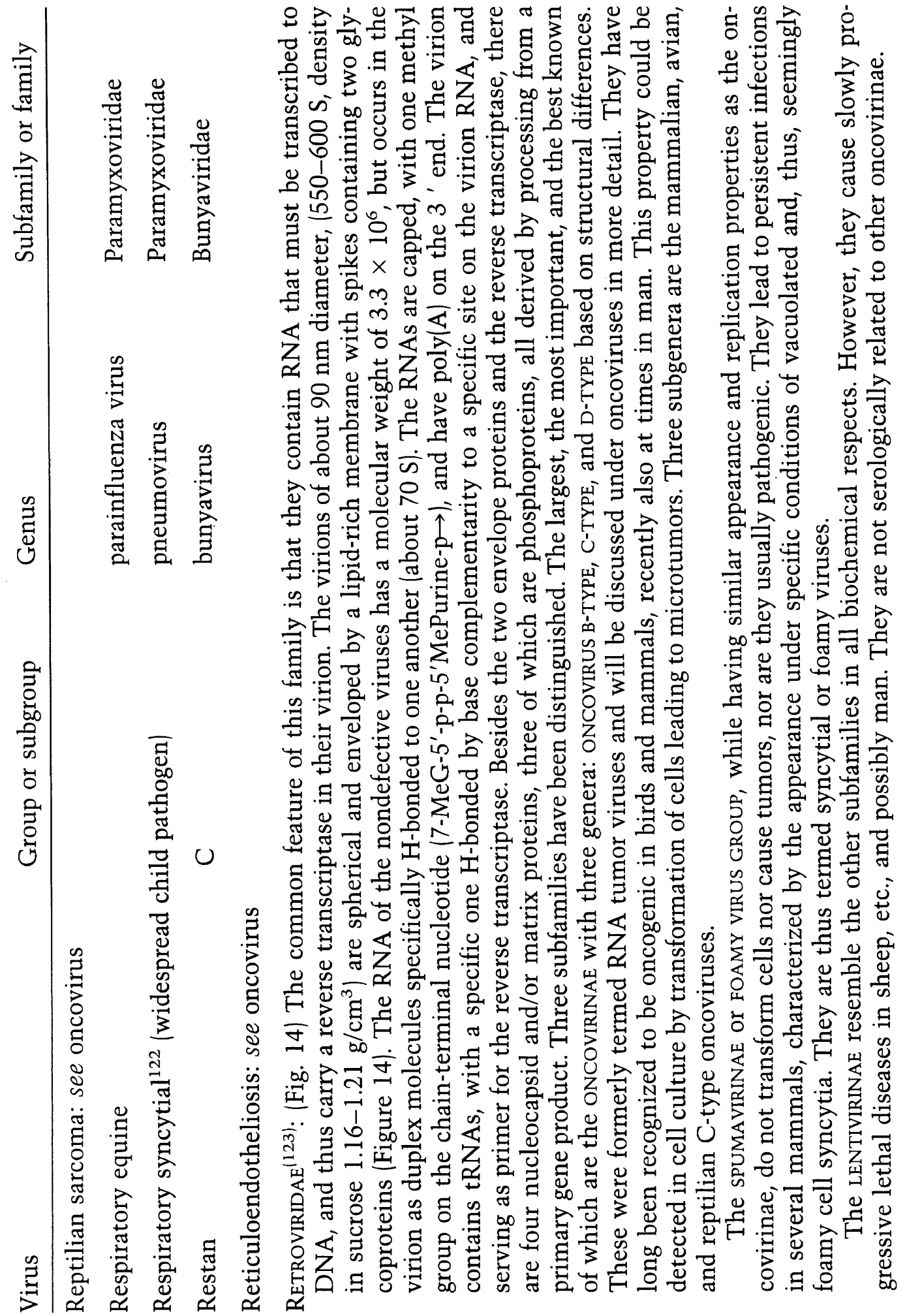




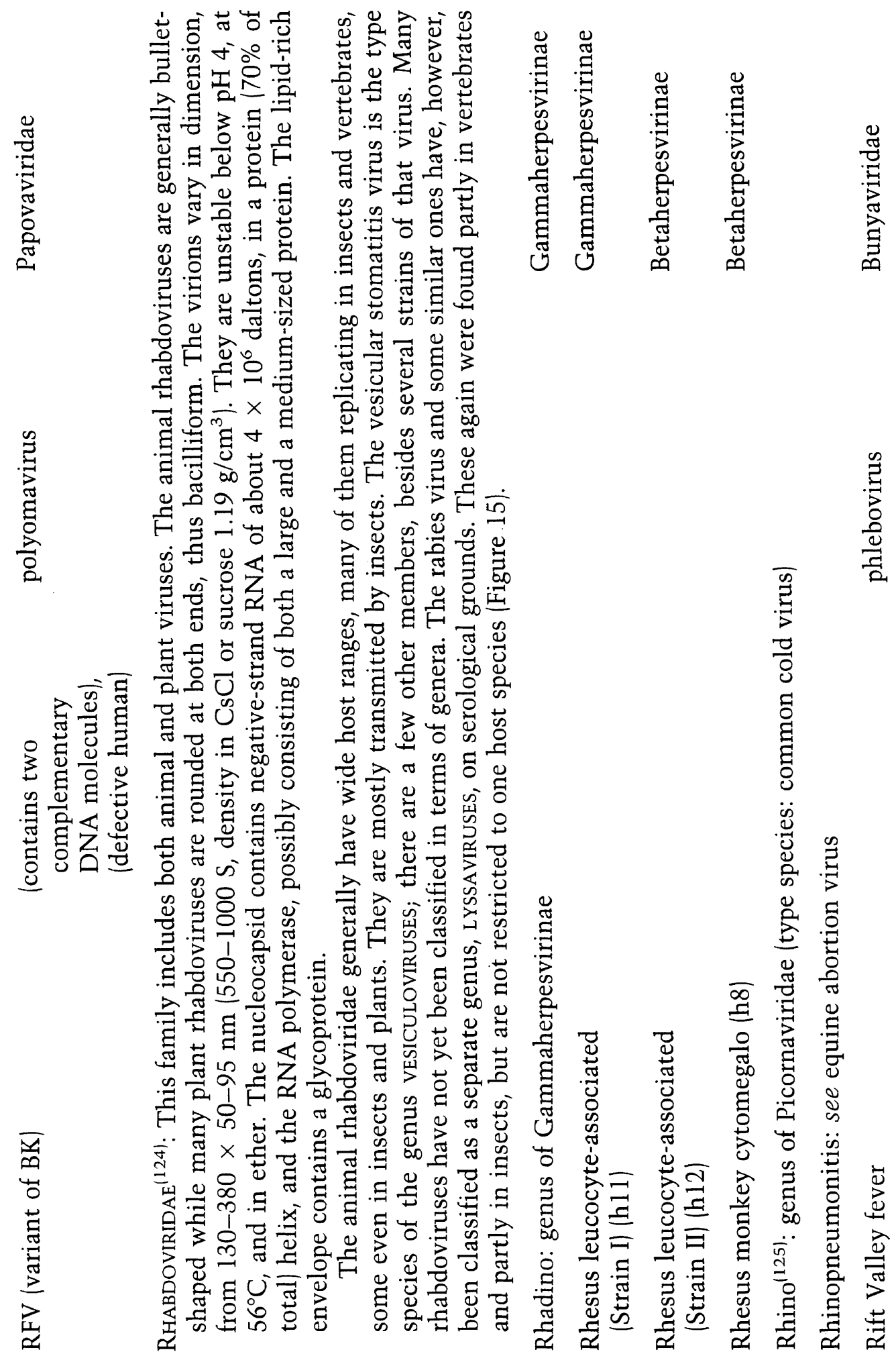



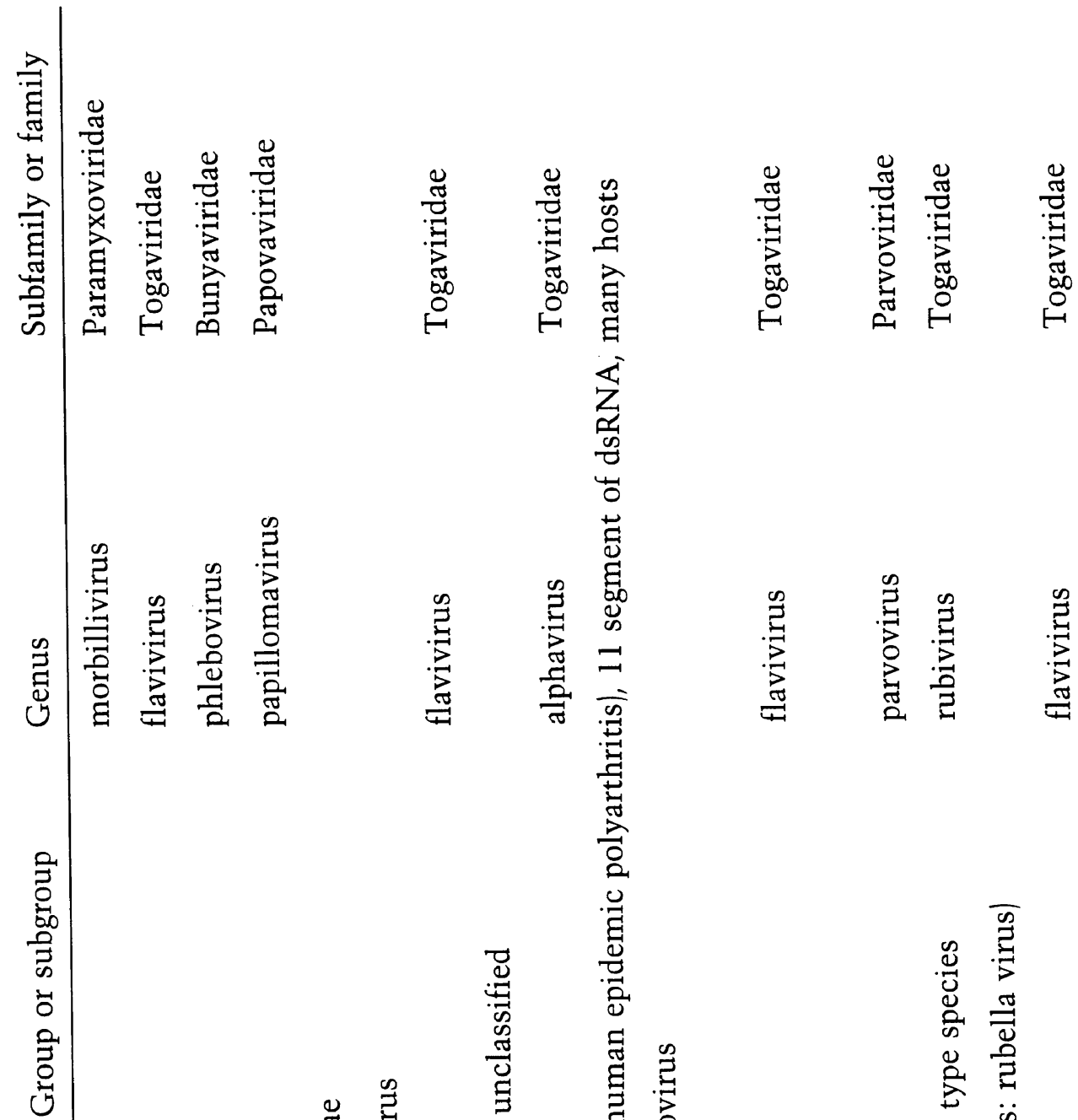


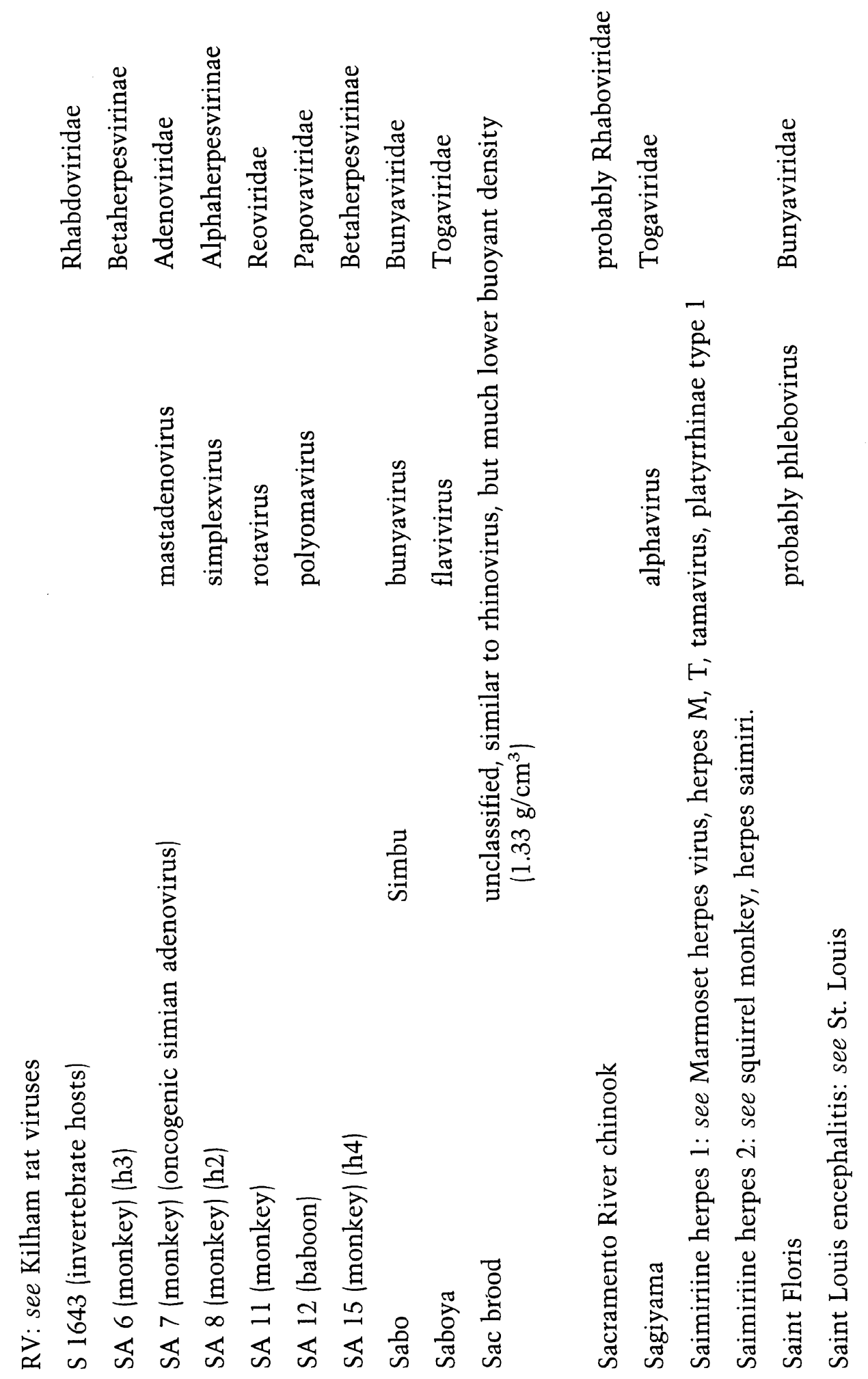



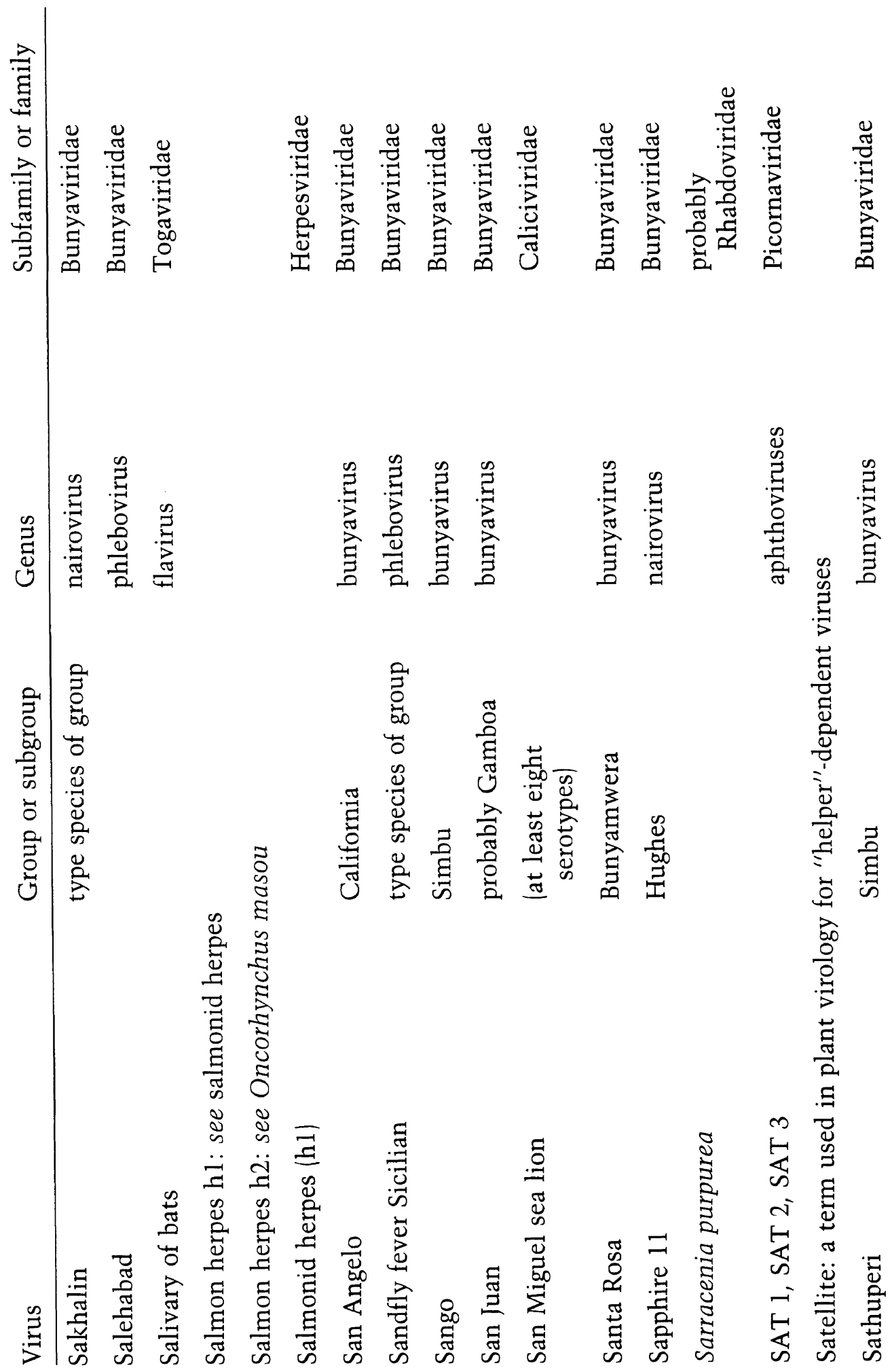

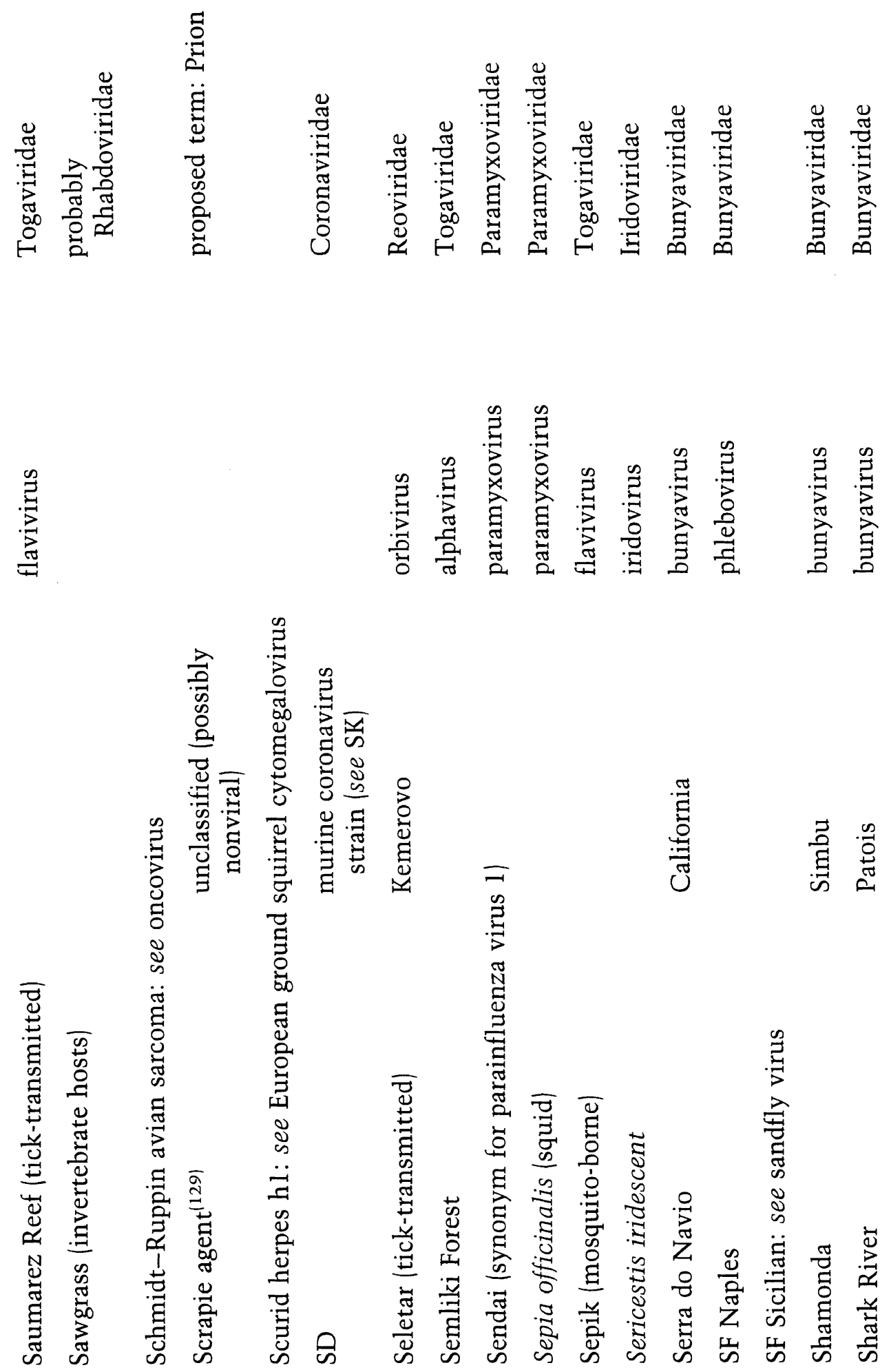

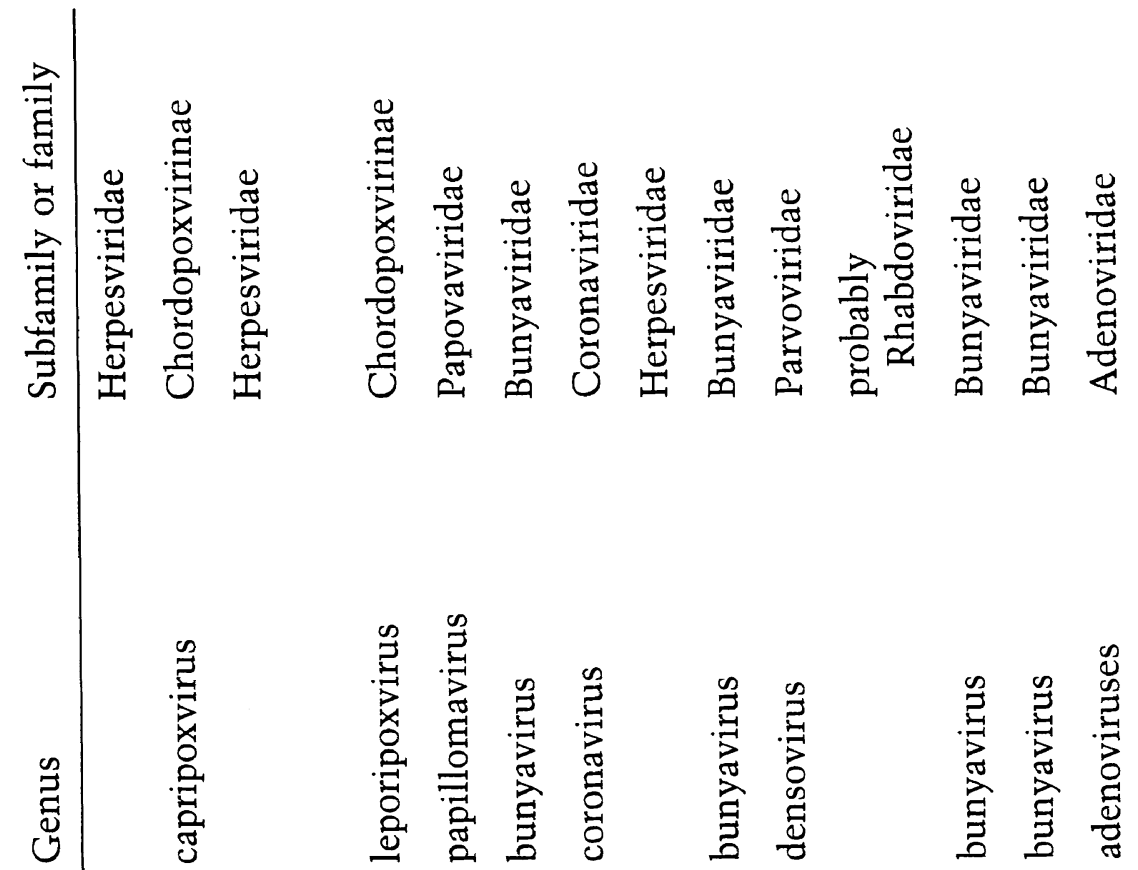

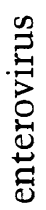

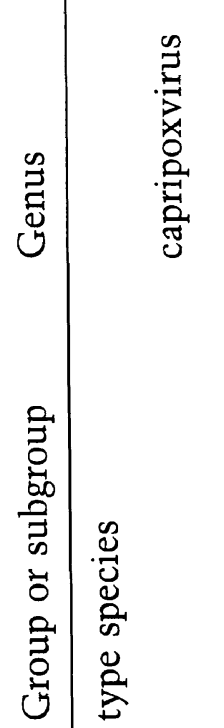

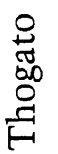

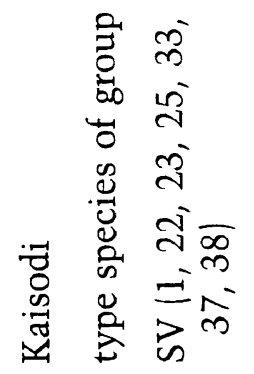

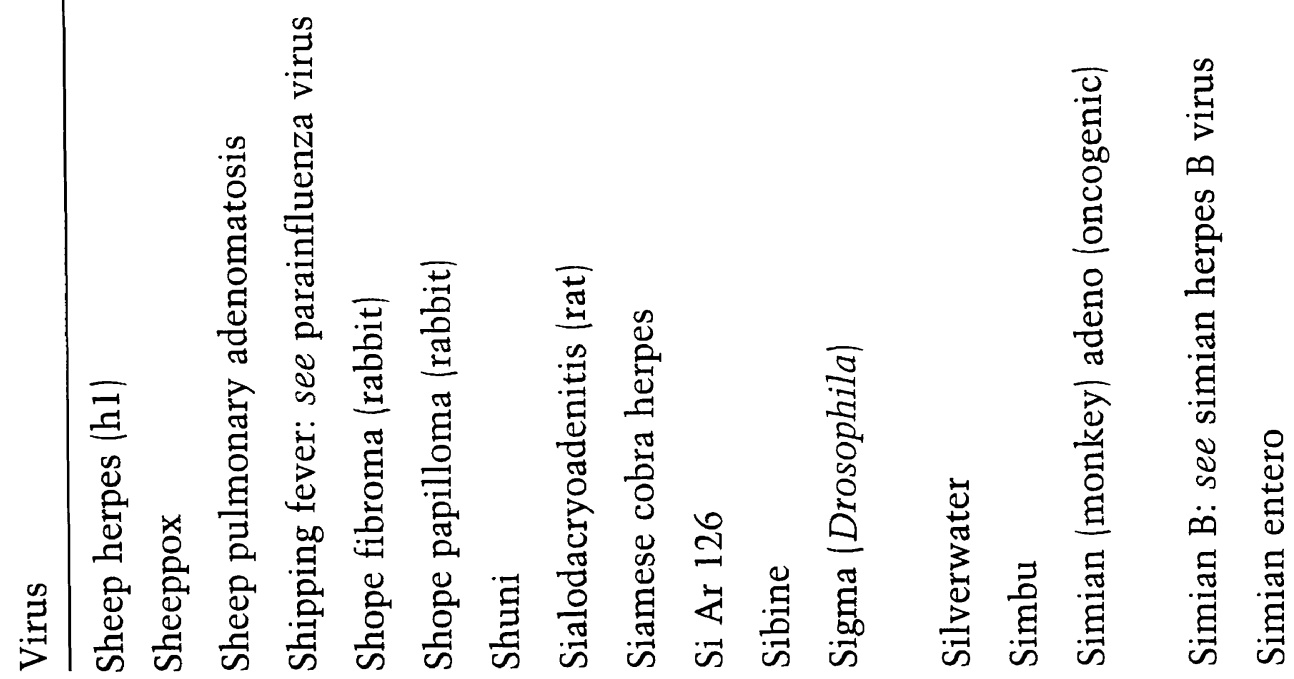



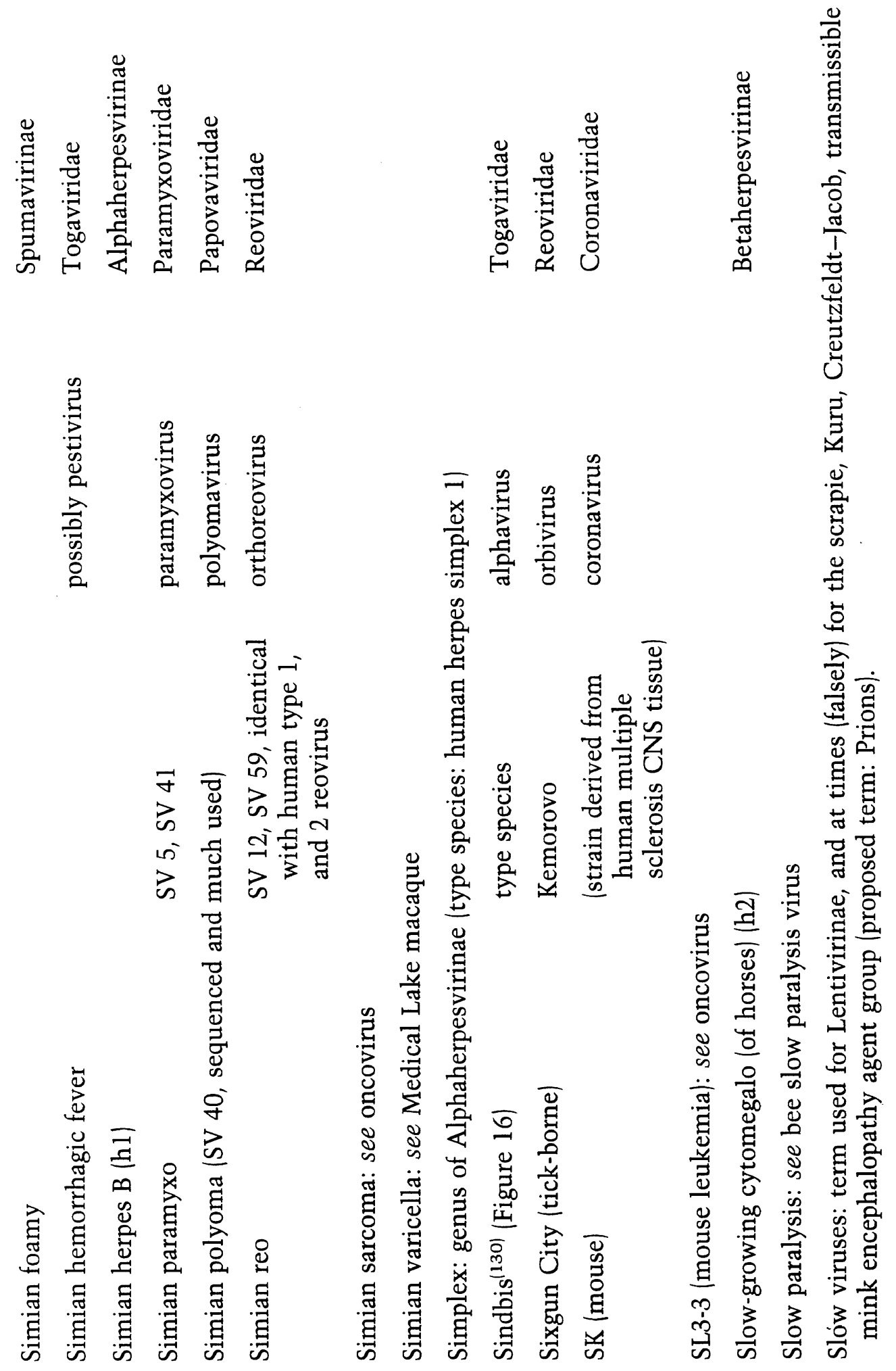


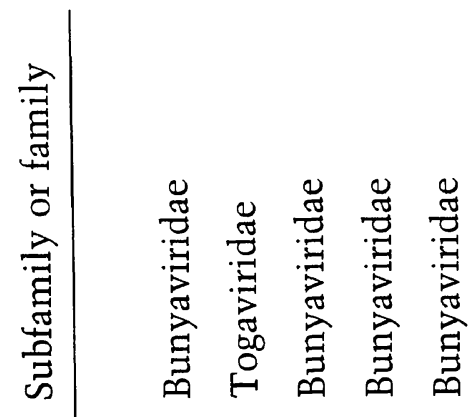

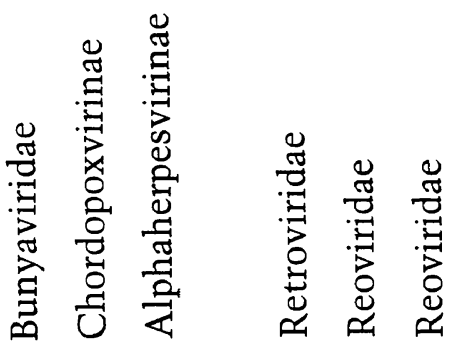

苞

疍

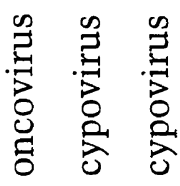

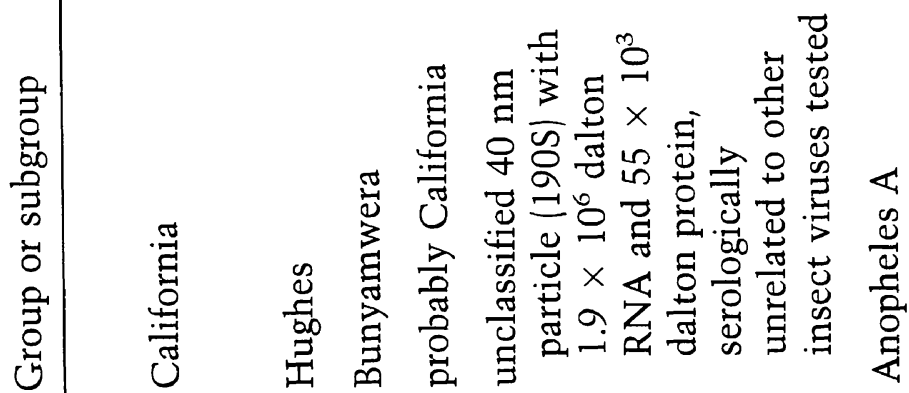

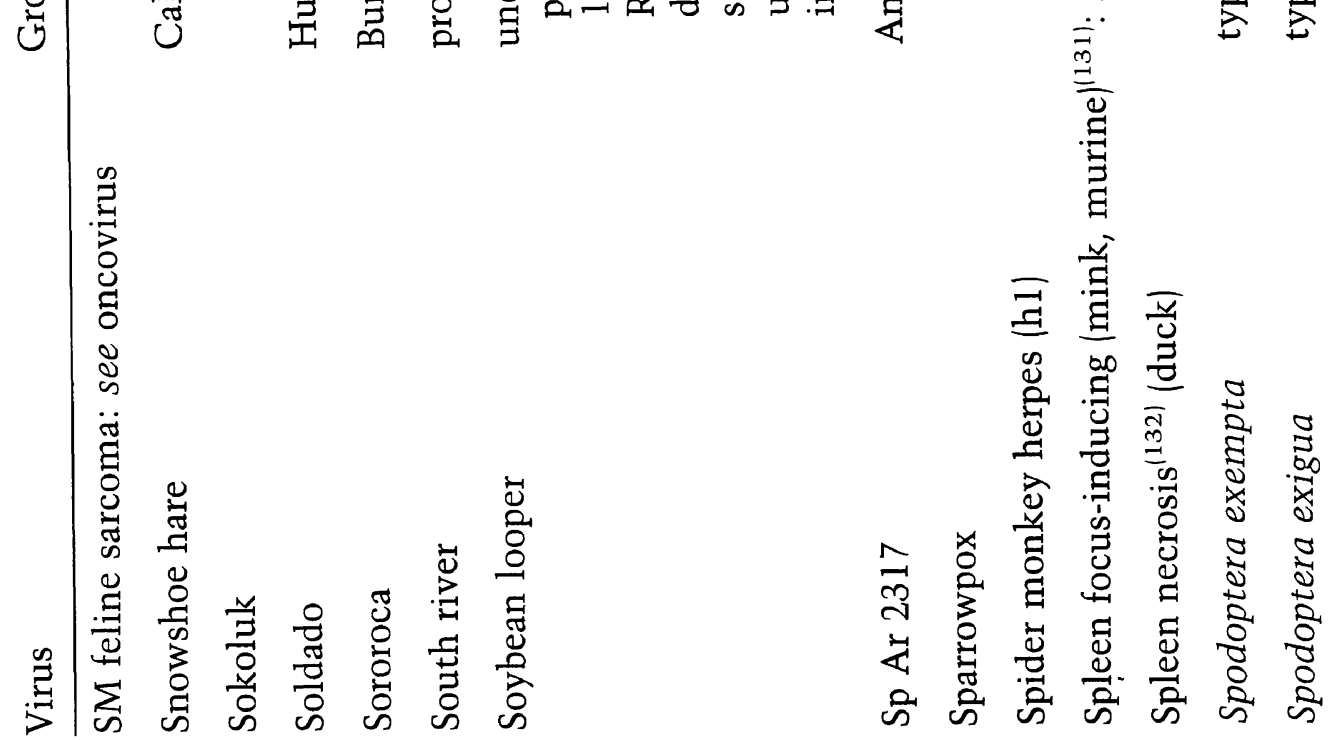



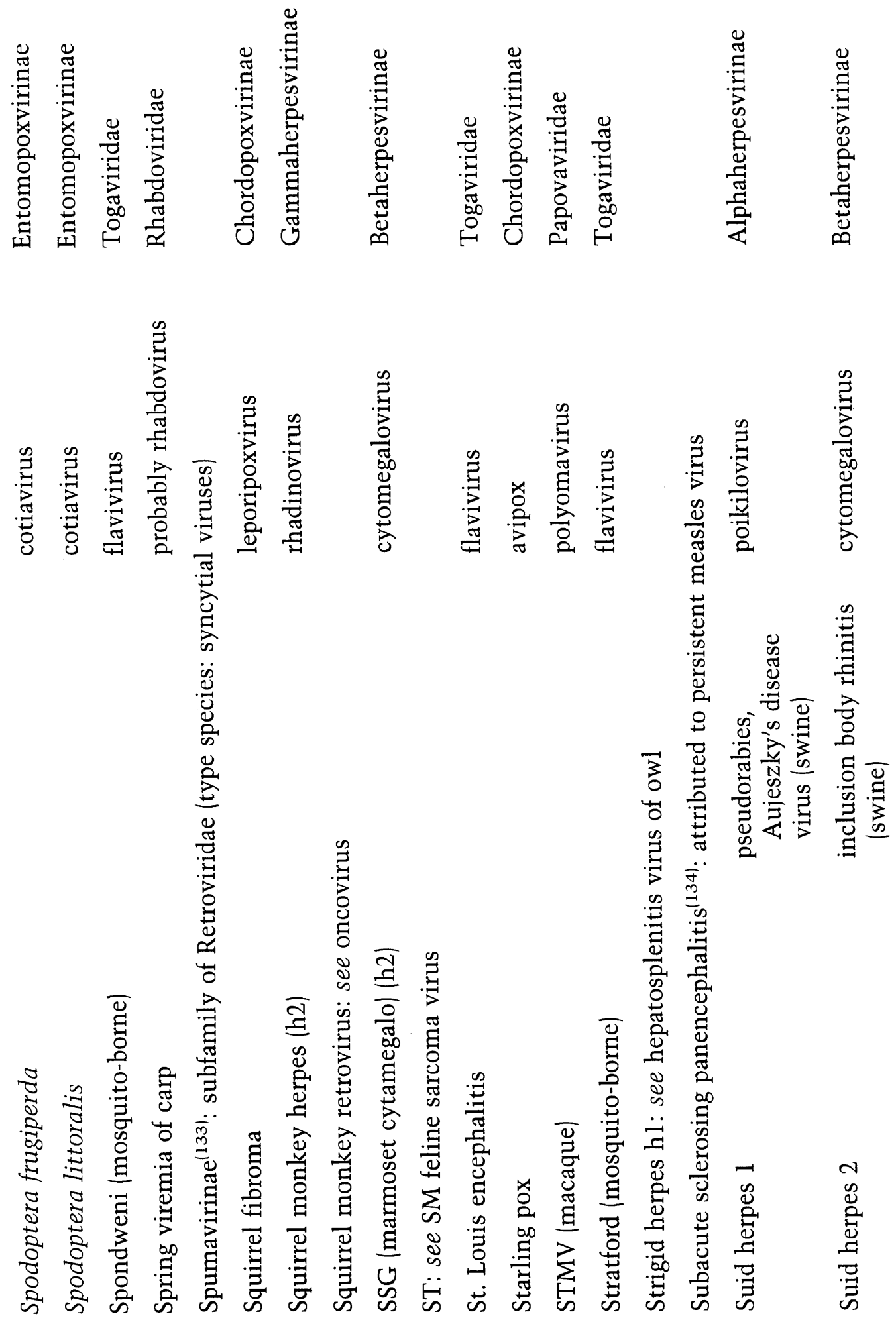


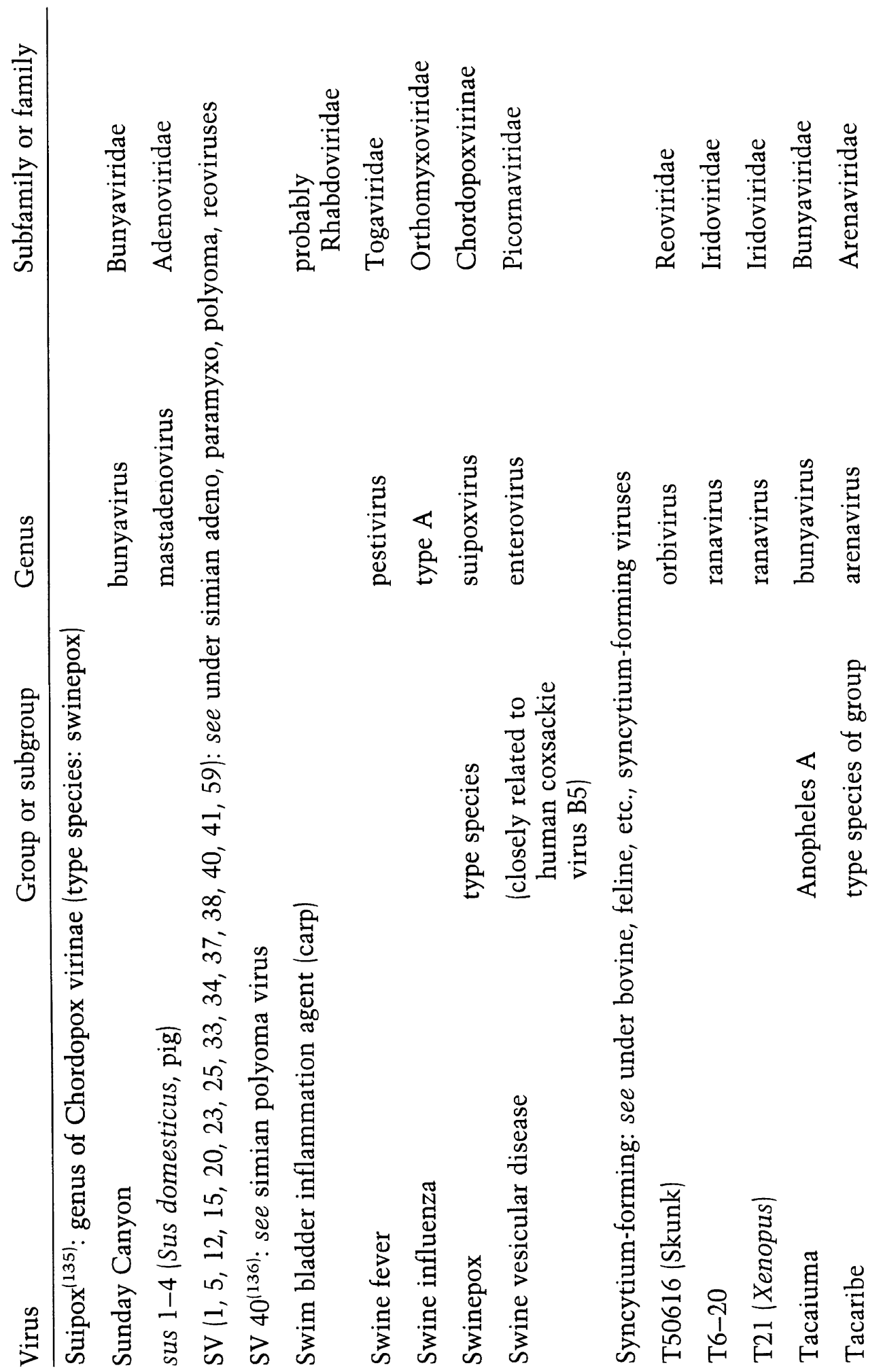



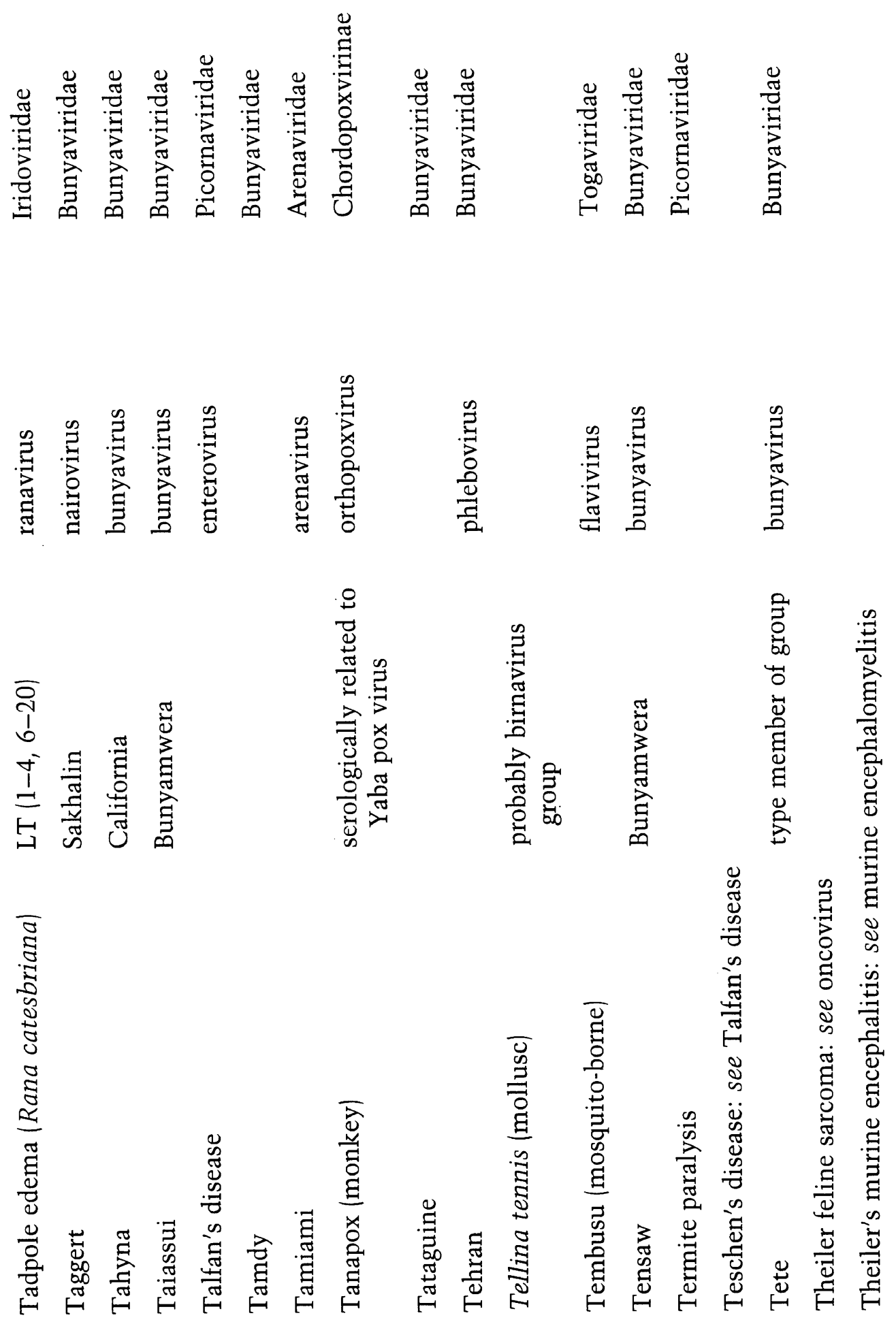


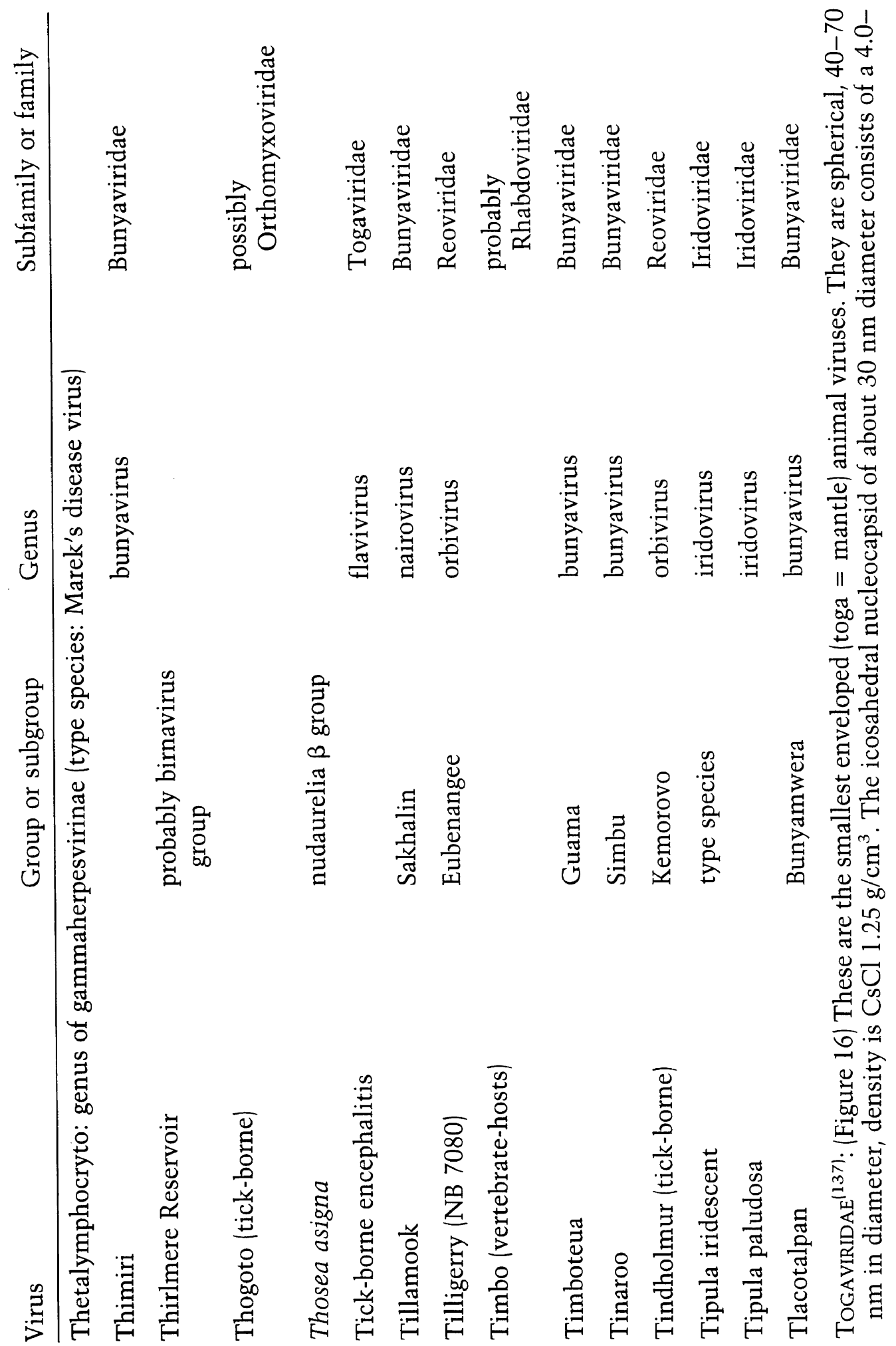




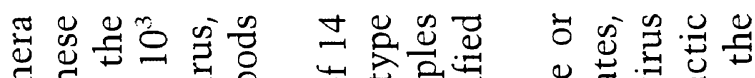

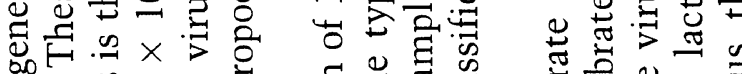

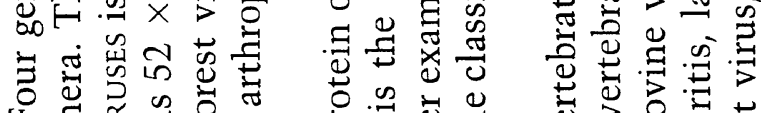

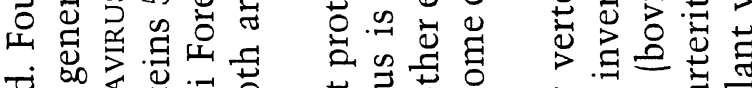

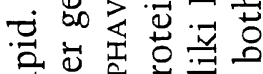

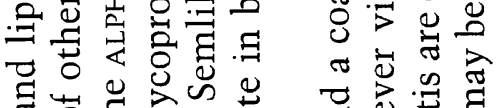
ते

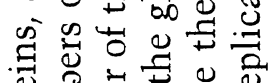

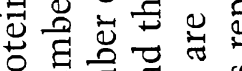

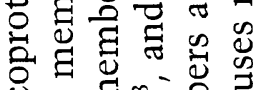

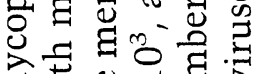
का

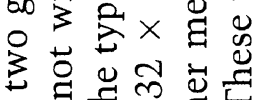
पै द्ञा

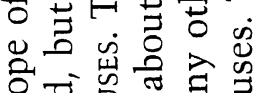

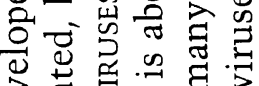

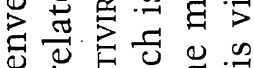
0 氖总 考䓵

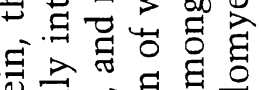

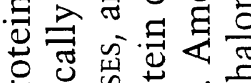
है.

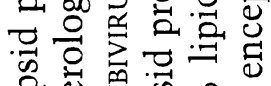

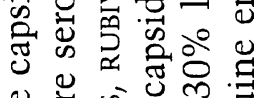

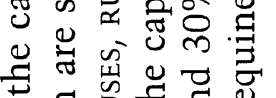
8 亭 든

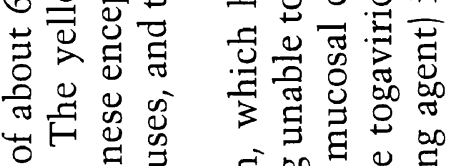

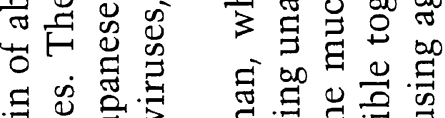

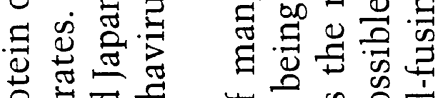

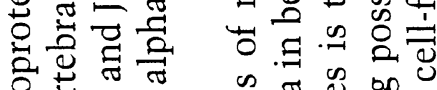

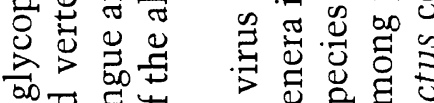
o.

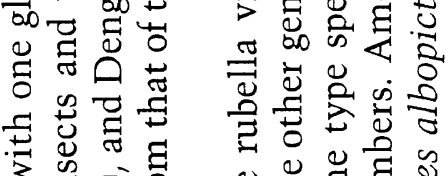

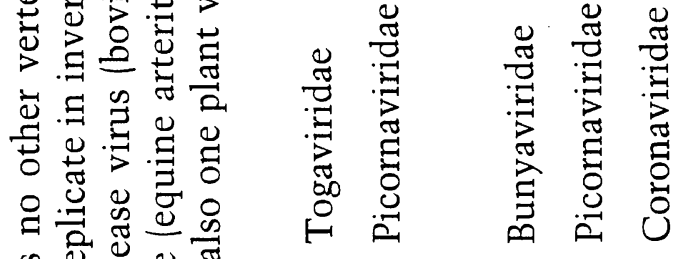

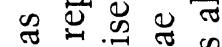

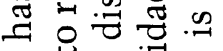
政

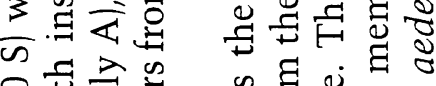

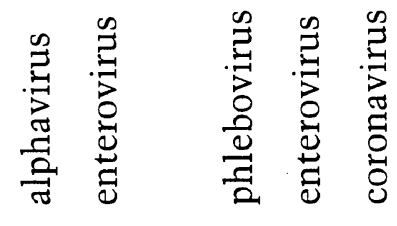

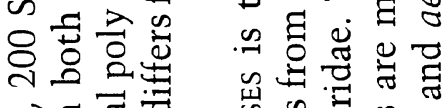

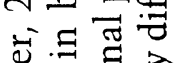

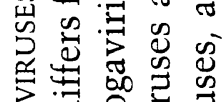

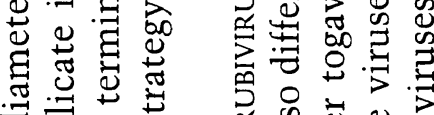
承:

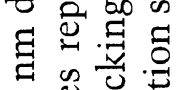

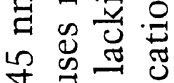

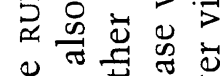

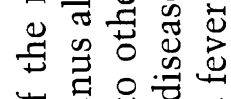

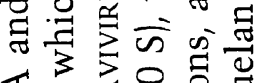

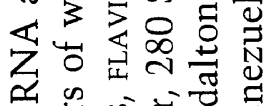

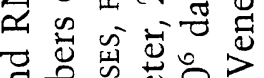

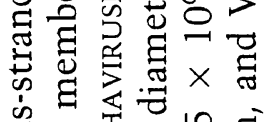

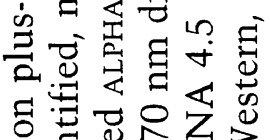
子

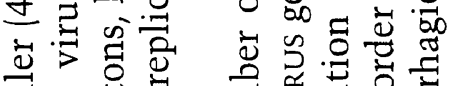
可语

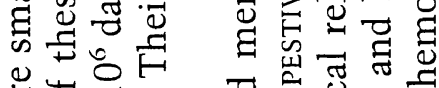

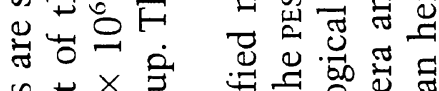

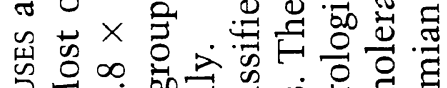

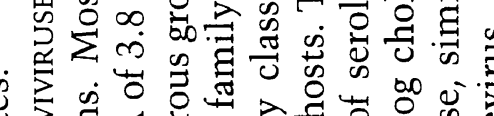

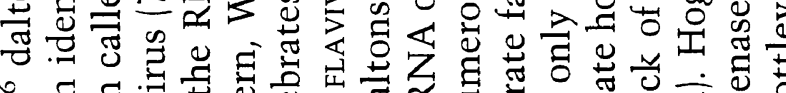

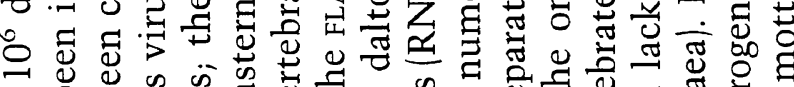

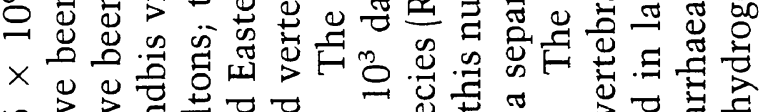

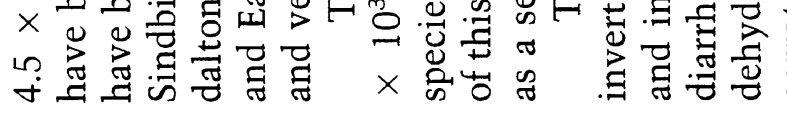




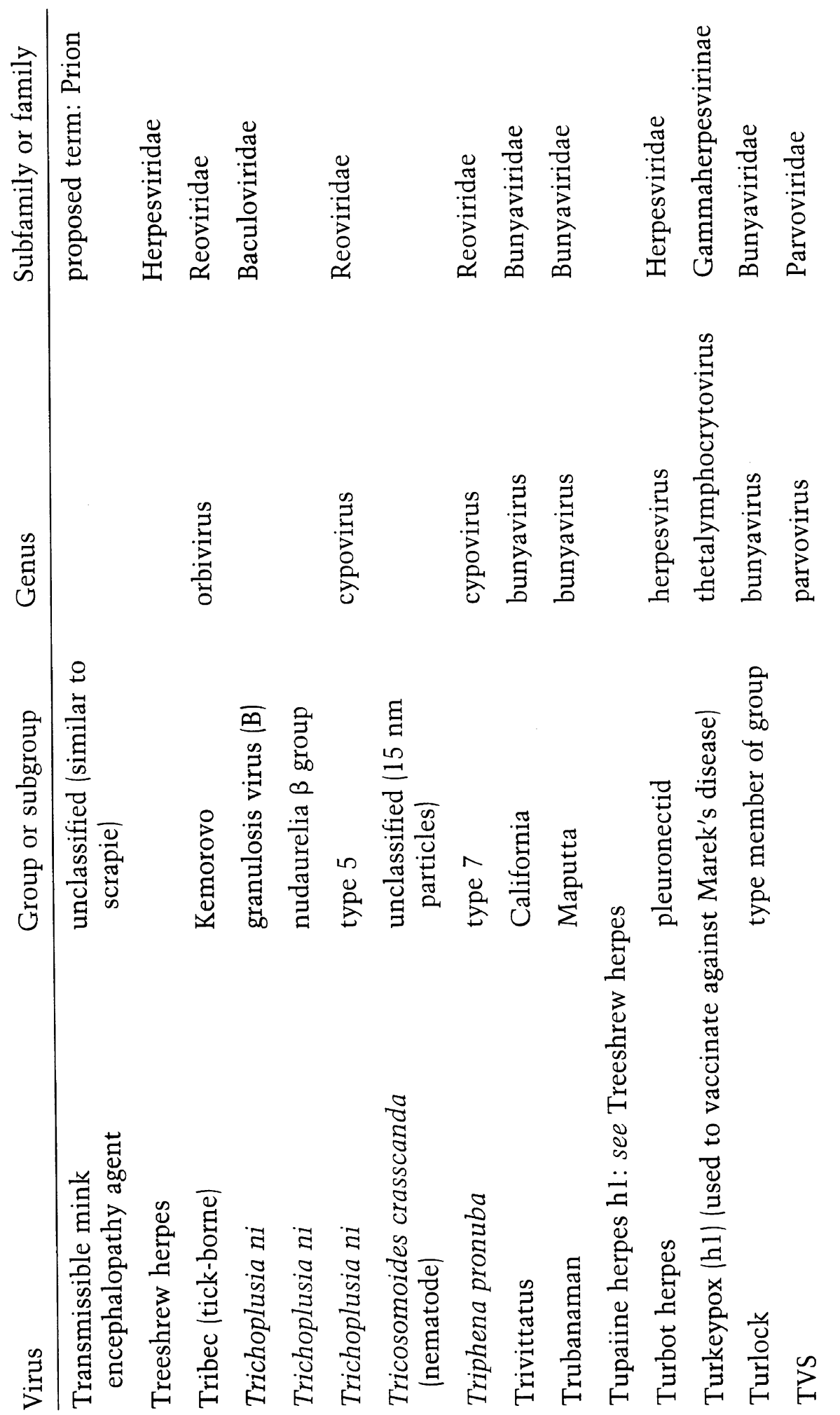



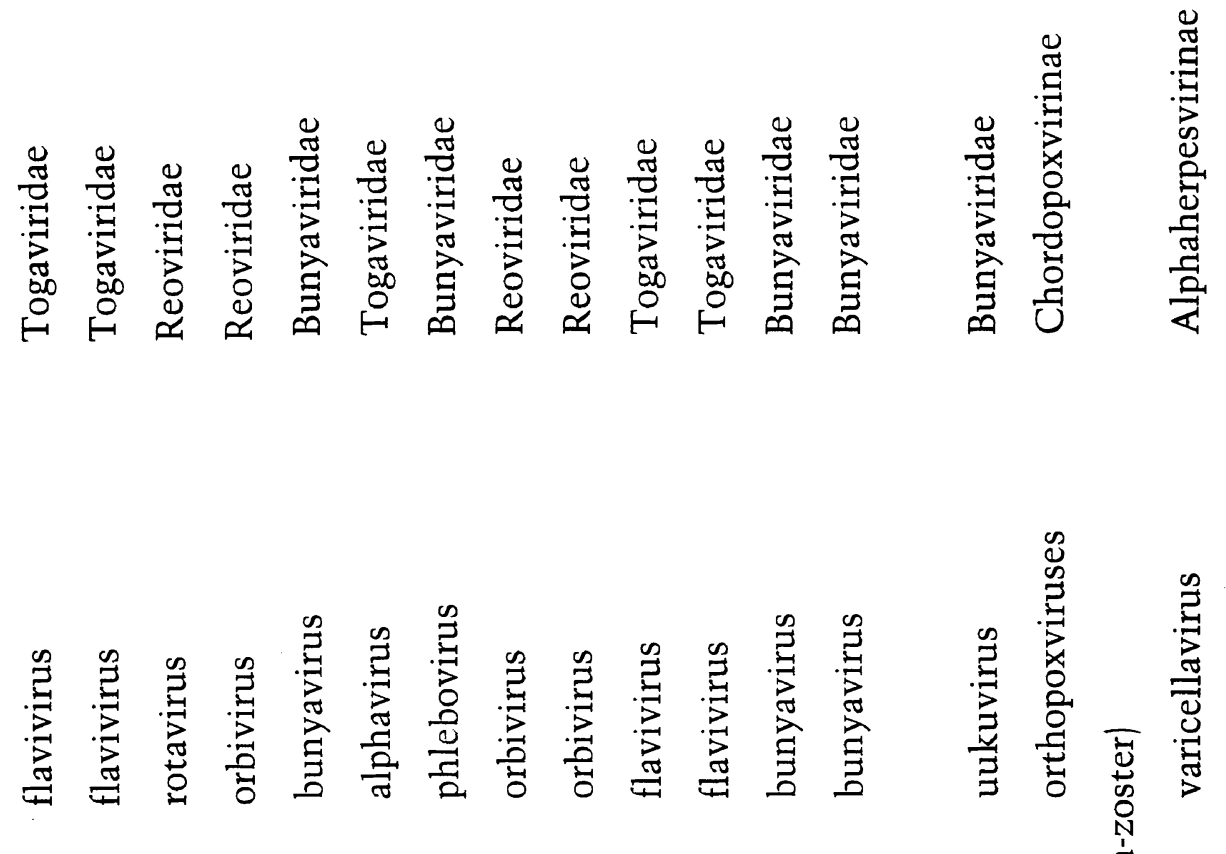

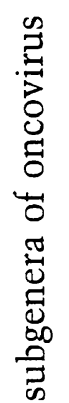

$\underset{\ddot{O}}{\stackrel{0}{\Xi}}$

官

苔 步

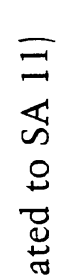

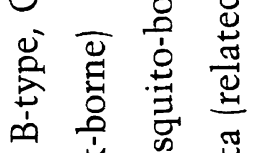

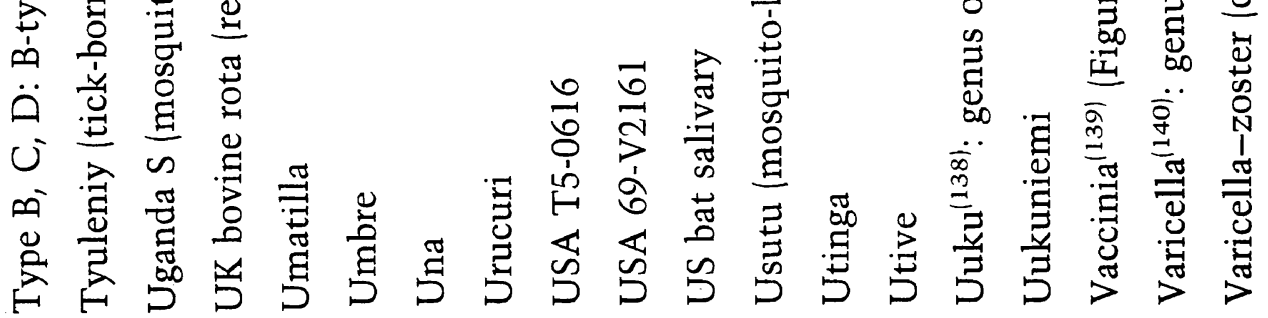

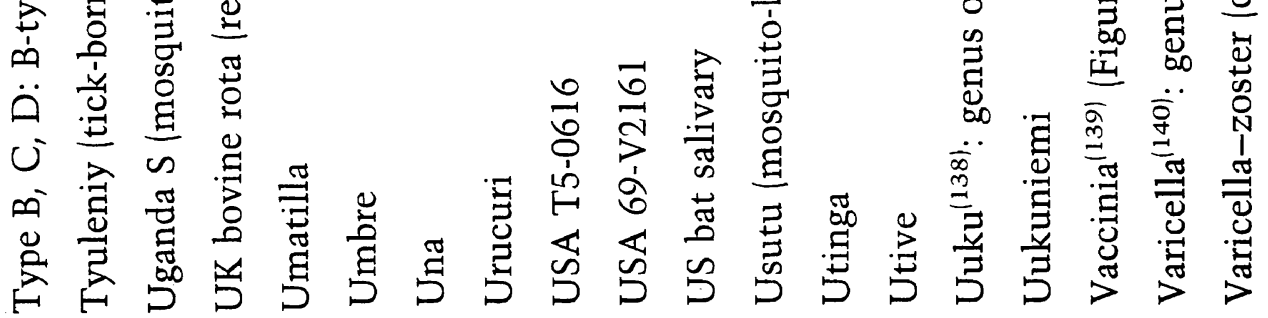



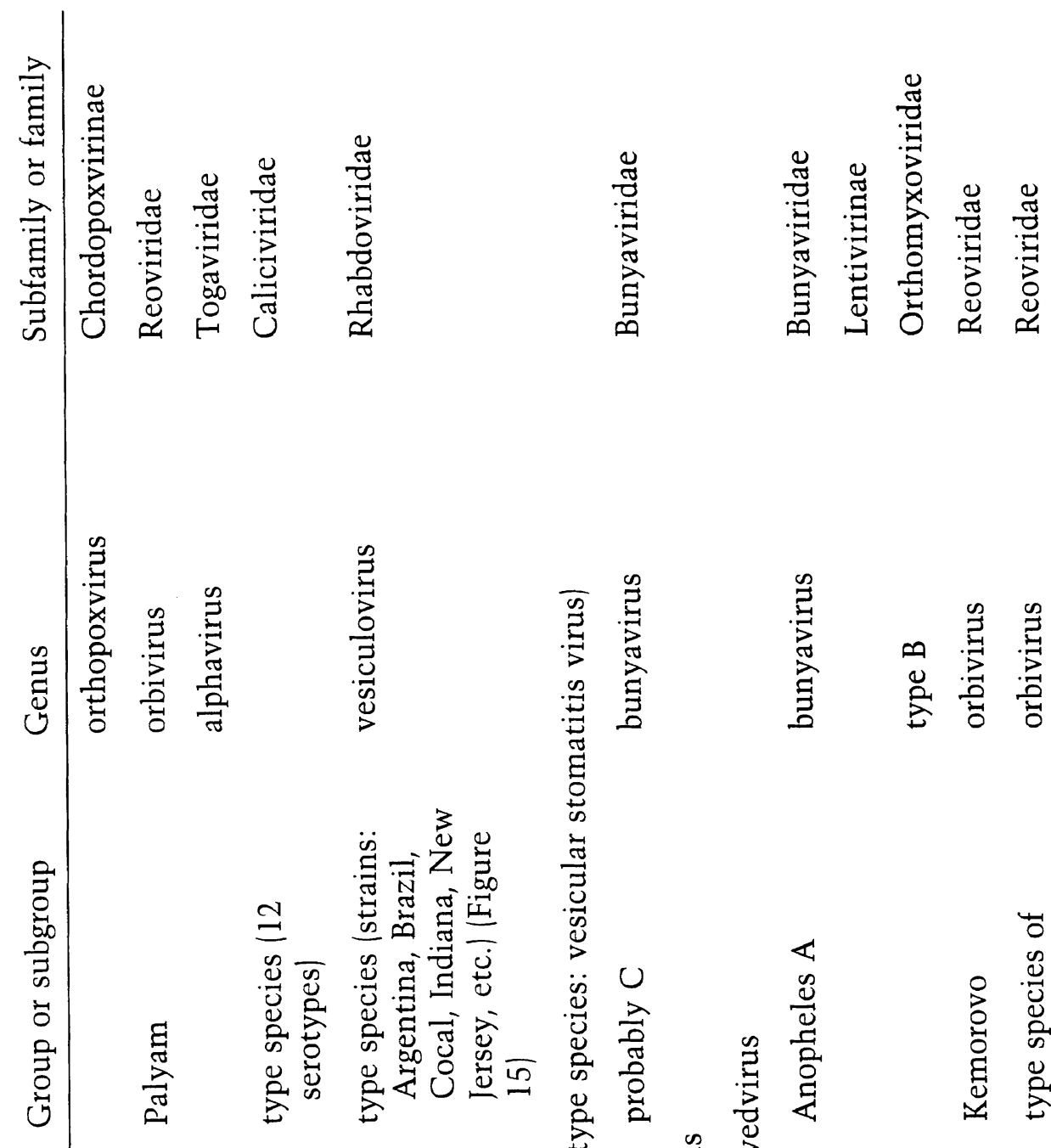

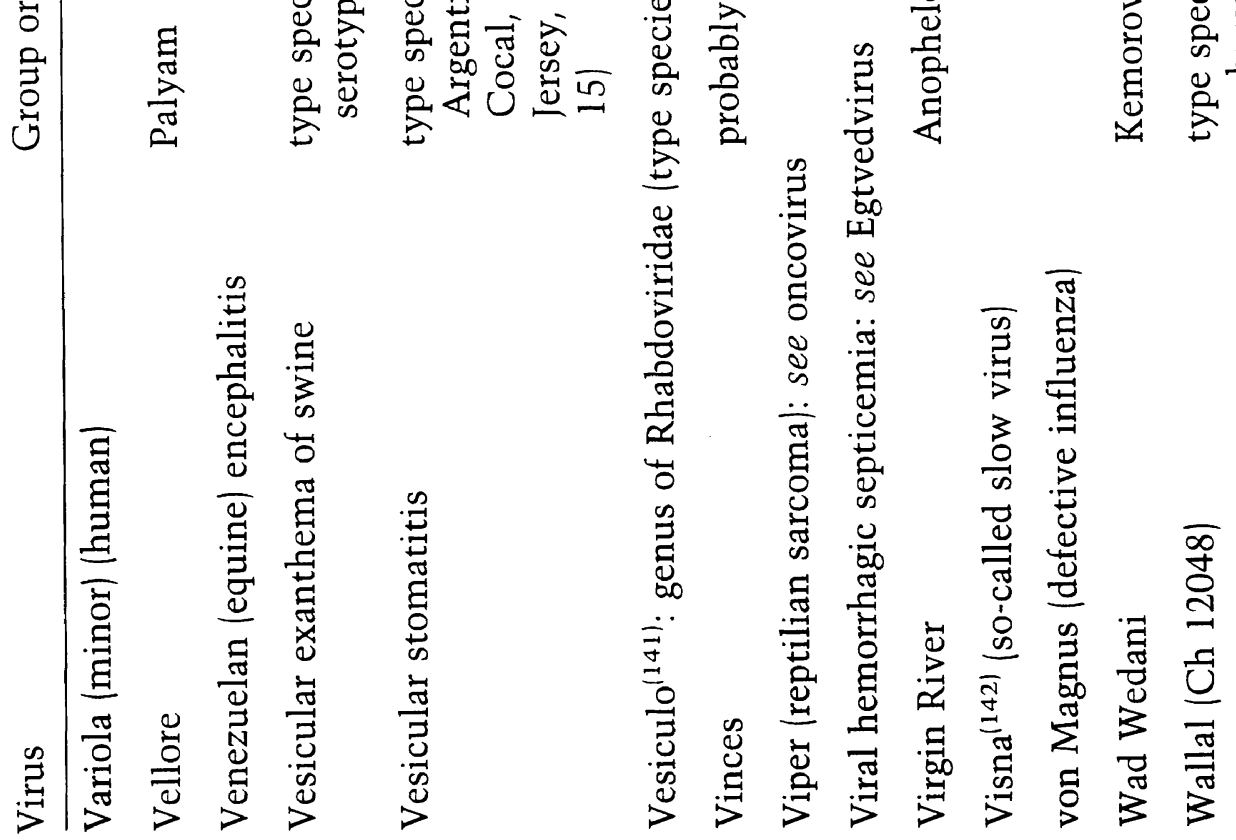




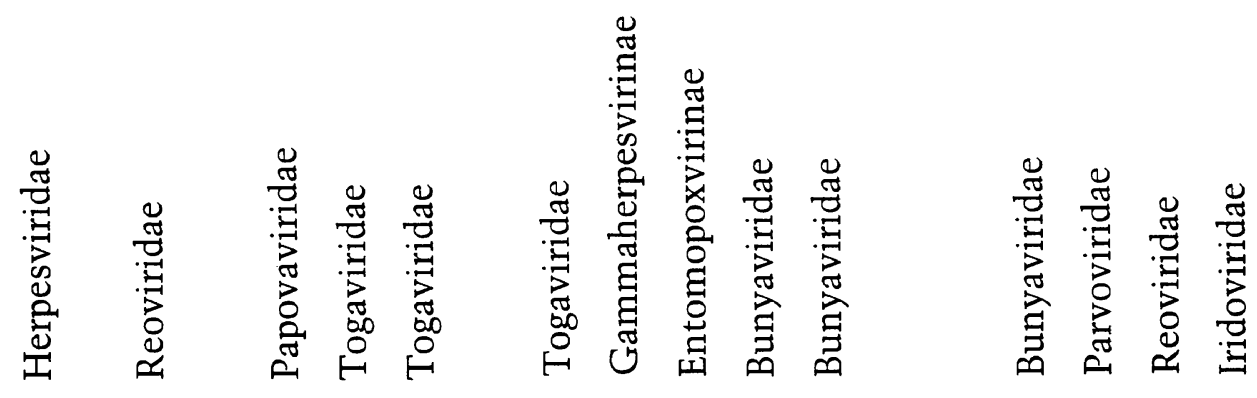

营
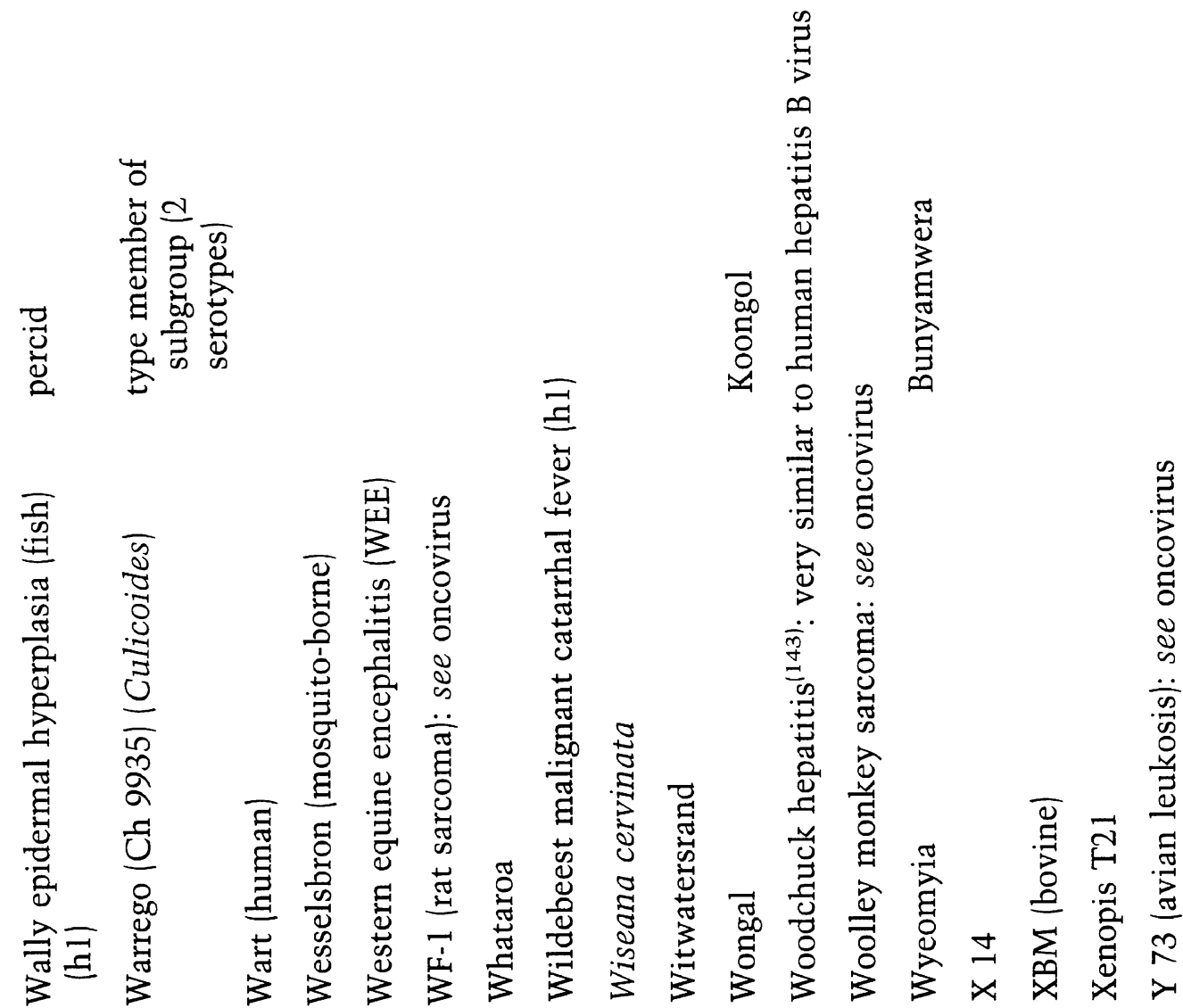


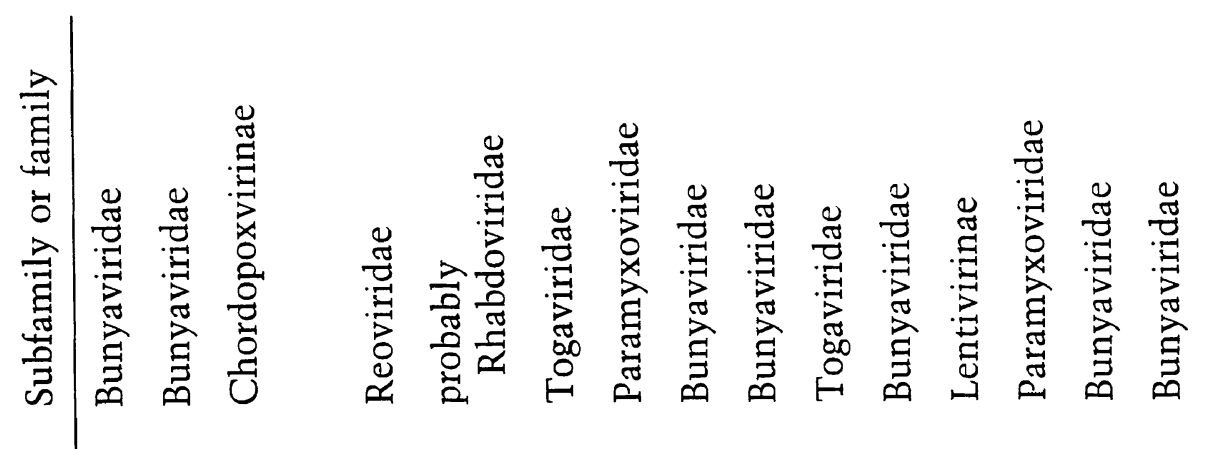

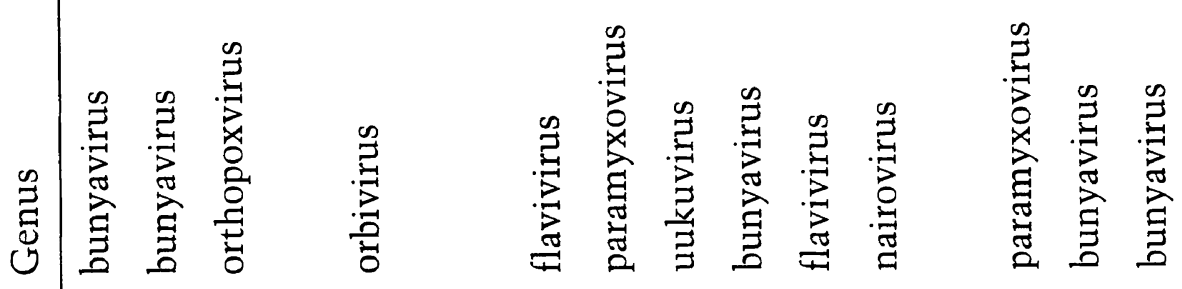

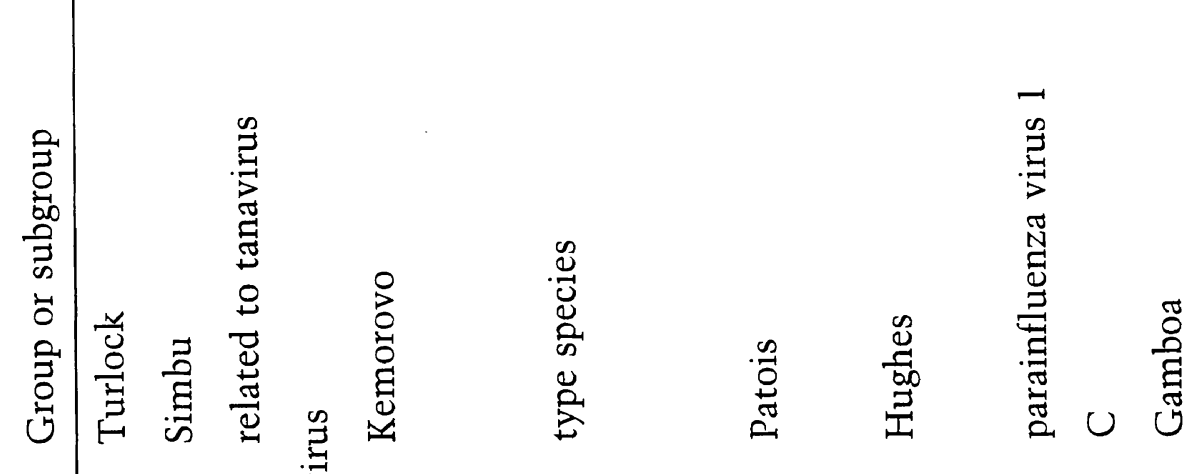

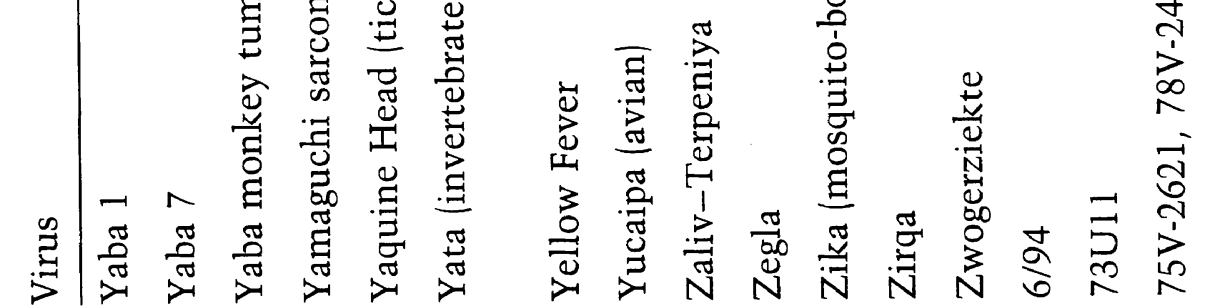




\section{References (Section I)}

1. Goff, P., Gilboa, E., Witte, O. N., and Baltimore, I., 1980, Structure of the Abelson murine leukemia virus genome and the homologous cellular gene: Studies with cloned viral DNA, Cell 22:727.

2. Grose, C., and Horwitz, M. S., 1976, Characterization of an enterovirus associated with acute infectious lymphocytosis. J. Gen. Virol. 30:347-355.

3a. Berns, K. I., and Hauswirth, W. W., 1979, Adeno-associated viruses, Adv. Virus Res. 25:407.

3b. Matthews, R. E. F., 1982, Classification and nomenclature of viruses, Intervirology 17:73.

4a. Philipson, L., and Lindberg, U., 1974, Reproduction of adenoviruses, in: Comprehensive Virology, Vol. 2 (H. Fraenkel-Conrat and R. R. Wagner, eds.), p. 143, Plenum Press, New York.

4b. Ginsberg, H. S., 1979, Adenovirus structural proteins, in: Comprehensive Virology, Vol. 13 (H. Fraenkel-Conrat and R. R. Wagner, eds.), p. 409, Plenum Press, New York.

4c. Ginsberg, H. S., and Young, C. S. H., 1979, Genetics of adenoviruses, in: Comprehensive Virology, Vol. 9 (H. Fraenkel-Conrat and R. R. Wagner, eds.), p. 27, Plenum Press, New York.

4d. Patch, C. T., Levine, A. S., and Lewis, A. M., Jr., 1979, The adenovirus-SV40 hybrid viruses, in: Comprehensive Virology, Vol. 13 (H. Fraenkel-Conrat and R. R. Wagner, eds.), p. 459, Plenum Press, New York.

4e. Matthews, R. E. F., 1982, Classification and nomenclature of viruses, Intervirology 17:59.

4f. Wigand et al., 1982, Adenoviridae: Second report, Intervirology 18:169.

5. Jurkovičová, M., Van Touw, J. H., Sussenbach, J. S., and Ter Schegget, J., 1979, Characterization of the nuclear polyhedrosis virus DNA of Adoxophyes orana and of Barathra brassicae, Virology 93:8.

6a. Salas, M. L., Kuznar, I., and Vinuela, E., 1981, Polyadenylation, methylation, and capping of the RNA synthesized in vitro by African swine fever virus, Virology 113:484.

6b. Goorha, R., and Granoff, A., 1979, Icosahedral cytoplasmic deoxyriboviruses, in: Comprehensive Virology, Vol. 14 (H. Fraenkel-Conrat and R. R. Wagner, eds.), p. 347, Plenum Press, New York.

7a. Shahrabadi, M. S., Cho, H. J., and Marusyk, R. G., 1977, Characterization of the protein and nucleic acid of Aleutian disease virus, $J$. Virol. 23:353.

7b. Porter, D. D., and Cho, H. J., 1980, Aleutian disease of mink: A model for persistent infection, in: Comprehensive Virology, Vol. 16 (H. Fraenkel-Conrat and R. R. Wagner eds.), p. 233, Plenum Press, New York.

8. Matthews, R. E. F., 1982, Classification and nomenclature of viruses, Intervirology 17:97.

9. Matthews, R. E. F., 1982, Classification and nomenclature of viruses, Intervirology $17: 48$. 
10. D'Arcy, C. J., Burnett, P. A., Hewings, A. D., and Goodman, R. M., 1981, Purification and characterization of a virus from the aphid Rhopalosiphum padi, Virology 112:346.

11. Matthews, R. E. F., 1982, Classification and nomenclature of viruses, Intervirology $17: 130$.

12a. Rawls, W. E., and Leung, W-C., 1979, Arenaviruses, in: Comprehensive Virology, Vol. 14 (H. Fraenkel-Conrat and R. R. Wagner eds.), p. 157, Plenum Press, New York.

12b. Matthews, R. E. F., 1982, Classification and nomenclature of viruses, Intervirology 17:119.

13. Bailey, L., and Woods, R. D., 1974, Three previously undescribed viruses for the honey bee, J. Gen. Virol. 25:175.

14. Herring, A. J., Gray, E. W., and Snodgrass, D. R., 1981, Identification and characterization of ovine astrovirus, J. Gen. Virol. 53:47.

15. Miller, L. K., and Dawes, K. P., 1979, Physical map of the DNA genome of Autographa californica nuclear polyhedrosis virus, J. Virol. 29(March):1044.

16. Davies, H. A., Dourmashkin, R. R., and MacNaughton, M. R., 1981, Ribonucleoprotein of avian infectious bronchitis virus, J. Gen. Virol. 53:67.

17a. Czernilofsky, A. P., Levinson, A. D., Varmus, H. E., and Bishop, J. M., 1980, Nucleotide sequence of an avian sarcoma virus oncogene $(s r c)$ and proposed amino acid sequence for gene product, Nature 287.

17b. Erikson, E., Collett, M. S., and Erikson, R. L., 1978, In vitro synthesis of a functional avian sarcoma virus transforming-gene product, Nature 274(August).

18. Matthews, R. E. F., 1982, Classification and nomenclature of viruses, Intervirology 17:43.

19a. Matthews, R. E. F., 1982, Classification and nomenclature of viruses, Intervirology 17:53.

19b. Tinsley, T. W., and Harrap, K. A., 1978, Viruses of invertebrates, in: Comprehensive Virology, Vol. 12 (H. Fraenkel-Conrat and R. R. Wagner, eds.), p. 1, Plenum Press, New York.

19c. Kelly, D. C., Lescott, T., Ayres, M. E., Carey, D., Coutts, A., and Harrap, K. A., 1981, Induction of a nonoccluded baculovirus persistently infecting Heliothis zea by $\mathrm{He}$ liothis armigera and Trichoplusia ni nuclear polyhedrosis viruses, Virology 112:174.

19d. Langridge, W. H. R., 1981, Biochemical properties of a persistent nonoccluded baculovirus isolated from Heliothis zea cells, Virology 112:770.

19e. Revet, B. M. J., and Guelpa, B., The genome of a baculovirus infecting Tipula paludosa (Meig) (diptera): A high molecular weight closed circular DNA of zero superhelix density.

19f. McCarthy, W. J., Mercer, W. E., and Murphy, T. F., 1978, Characterization of the DNA from four heliothis nuclear polyhedrosis virus isolates, Virology 90:374.

20a. Bailey, L., 1976, Viruses attacking the honey bee, Adv. Virus Res. 20:271.

20b. Bailey, L., Carpenter, J. M., and Woods, R. D., 1981, Properties of a filamentous virus of the honey bee (Apis mellifera), Virology 114:1.

20c. Bailey, L., Carpenter, J. M., Govier, D. A., and Woods, R. D., 1980, Bee virus X, $I$. Gen. Virol. 51:405.

20d. Longworth, J. F., 1978, Small isometric viruses of invertebrates, Adv. Virus Res. 23:103.

21. McPhee, D. A., and Westaway, E. G., 1981, Comparisons of belmont virus, a possible bunyavirus unique to Australia, with bunyamwera virus, J. Gen. Virol. 54:135.

22. Weiss, M., Steck, F., and Horzinek, M. C., 1983, Purification and partial characterization of a new enveloped RNA virus (berne virus), J. Gen. Virol. 64:1849.

23. Matthews, R. E. F., 1982, Classification and nomenclature of viruses, Intervirology 17:49.

24. MacDonald, R. D., and Gower, D. A., 1981, Geno and phenotypic divergence among three serotypes of aquatic birnaviruses (infectious pancreatic necrosis virus), Virology 114: 187. 
25. Wright, P. J., and Di Mayorca, G., 1975, Virion polypeptide composition of the human papovavirus BK: Comparison with simian virus 40 and polyoma virus, J. Virol. 15:828.

26. Friesen, P. D., and Rueckert, R. R., 1982, Black beetle virus: Messenger for protein $B$ is a subgenomic viral RNA, $J$. Virol. 42:986.

27. Della-Porta, A. J., and Brown, F., 1979, Physicochemical characterization of bovine ephemeral fever virus as a member of the family Rhabdoviridae, J. Gen. Virol. 44:99.

28. Ludwig, H., 1983, Bovine herpesviruses in: The Viruses, Vol. II (H. Fraenkel-Conrat, R. R. Wagner, and B. Roizman eds.), p. 135, Plenum Press, New York.

29. Buchman, T. G., and Roizman, B., 1978, Anatomy of bovine mammillitis DNA: Restriction endonuclease maps of four populations of molecules that differ in the relative orientation of their long and short components, J. Virol. 25:395.

30. Pritchett, R., Manning, J. S., and Zee, Y. C., and Characterization of bovine viral diarrhea virus RNA, J. Virol. 15:1342.

31a. Bishop, D. H. L., and Shope, R. E., 1979, Bunyaviridae, in: Comprehensive Virology, Vol. 14 (H. Fraenkel-Conrat and R. R. Wagner, eds.), p. 1, Plenum Press, New York.

31b. Matthews, R. E. F., 1982, Classification and nomenclature of viruses, Intervirology 17:115.

31c. Beaty, B. J., Holterman, M., Tabachnick, W., and Shope, R. E., 1981, Molecular basis of bunyavirus transmission by mosquitoes: Role of the middle-sized RNA segment, Science 211:1433.

31d. Beaty, B. J., Miller, B. R., Shope, R. E., Rozhon, E. J., and Bishop, D. H. L., 1982, Molecular basis of bunyavirus per os infection of mosquitoes: Role of the middlesized RNA segment, Proc. Natl. Acad. Sci. USA 79:1295.

31e. Obijeski, J. F., and Murphy, F. A., 1977, Bunyaviridae: Recent biochemical developments, J. Gen. Virol. 37:1.

32. Bridger, J. C., and Woode, G. N., 1976, Characterization of two particle types of calf rotavirus, J. Gen. Virol. 31:245.

33a. Schaffer, F. L., 1979, Caliciviruses, in: Comprehensive Virology, Vol. 14 (H. FraenkelConrat and R. R. Wagner, eds.l, p. 249, Plenum Press, New York.

33b. Schaffer, F. L. et al., 1980, Caliciviridae, Intervirology 14:1.

33c. Matthews, R. E. F., 1982, Classification and nomenclature of viruses, Intervirology 17:133.

34a. Goldman, N., Presser, I., and Sreevalsan, T., 1977, California encephalitis virus: Some biological and biochemical properties, Virology 76:352.

34b. Vorndam, A. V., and Trent, D. W., 1979, Oligosaccharides of California encephalitis viruses, Virology 95:1.

35. Parrish, C. R., and Carmichael, L. E., 1983, Antigenic structure and variation of canine parvovirus type-2, feline panleukopenia virus, and mink enteritis virus, Virology 129:401.

36. Roberson, N. M., McGuire, T. C., Klevjer-Anderson, P., Gorham, J. R., and Cheevers, W. P., 1982, Caprine arthritis-encephalitis virus is distinct from visna and progressive pneumonia viruses as measured by genome sequence homology, I. Virol. 44:755.

37. Matthews, R. E. F., 1982, The capripoxviruses, Intervirology 17:44.

38. Matthews, R. E. F., 1982, The cardioviruses, Intervirology 17:130.

39a. Dixon, R. A. F., and Farber, F. E., 1980, Channel catfish virus: Physicochemical properties of the viral genome and identification of viral polypeptides, Virology 103:267278.

39b. McAllister, P. E., 1979, Fish viruses and viral infections, in: Comprehensive Virology, Vol. 14 (H. Fraenkel-Conrat and R. R. Wagner, eds.), p. 401, Plenum Press, New York.

40. Matthews, R. E. F., 1982, The chloriridoviruses, Intervirology 17:57.

41. Matthews, R. E. F., 1982, The chordopoxvirinae, Intervirology 17:42.

42a. Matthews, R. E. F., 1982, The Coronaviridae, Intervirology 17:102.

42b. Robb, J. A., and Bond, C. W., 1979, Coronaviridae, in: Comprehensive Virology, Vol. 14 (H. Fraenkel-Conrat and R. R. Wagner, eds.), p. 193, Plenum Press, New York. 
42c. Tyrell, D. A. J., Alexander, D. J., Almeida, J. D., Cunningham, C. H., Easterday, B. C., Garwes, D. J., Hierholzer, J. C., Kapikian, A., MacNaughton, M. R., and McIntosh, K., 1978, Coronaviridae: Second report, Intervirology 10:321-328.

42d. Mahy, B. W. J., 1980, Coronavirus comes of age, Nature 288:536.

42e. Dennis, D. E., and Brian, D. A., 1982, RNA-dependent RNA polymerase activity in coronavirus-infected cells, 42:153-164.

42f. Siddell, S. G., et al. 1983, Coronaviridae, Intervirology 20:181.

43. Ueda, Y., Dumbell, K. R., Tsuruhara, T., and Tagaya, I., 1978, Studies on cotia-an unclassified poxvirus, J. Gen. Virol. 40:263-276.

44a. Wolinsky, J. S., and Johnson, R. T., 1980, Role of viruses in chronic neurological diseases, in: Comprehensive Virology, Vol. 16 (H. Fraenkel-Conrat and R. R. Wagner, eds.), p. 257, Plenum Press, New York.

44b. Manuelidis, L., and Manuelidis, E. E., 1981, Search for specific DNAs in CreutzfeldtJakob infectious brain fractions using "nick translation," Virology 109:435.

44c. Gajdusek, D. C., 1977, Unconventional viruses and the origin and disappearance of kuru, Science 197:943.

45a. Eaton, B. T., and Steacie, A. D., 1980, Cricket paralysis virus RNA has a $3^{\prime}$ terminal poly(A), J. Gen. Virol. 50:167.

45b. Scotti, P. D., and Longworth, J. F., 1980, The biology and ecology of strains of an insect small RNA virus complex, Adv. Virus Res. 26:117.

46a. Matthews, R. E. F., 1982, Classification and nomenclature of viruses, Intervirology 17:49.

46b. Rapp, F., 1980, Persistence and transmission of cytomegalovirus, in: Comprehensive Virology, Vol. 16 (H. Fraenkel-Conrat and R. R. Wagner, eds.), p. 193, Plenum Press, New York.

46c. Stinski, M. F., 1983, Molecular biology of cytomegaloviruses, in: The Viruses, The Herpesviruses, Vol. II (H. Fraenkel-Conrat, R. R. Wagner, and B. Roizman, eds.), p. 67, Plenum Press, New York.

46d. Rapp, F. 1983, The biology of cytomegaloviruses, in: The Viruses, The Herpesviruses, Vol. II (H. Fraenkel-Conrat, R. R. Wagner, and B. Roizman, eds.), p. 1, Plenum Press, New York.

47a. Matthews, R. E. F., 1982, Classification and nomenclature of viruses, Intervirology 17:86.

47b. Payne, C. C., and Mertens, P. P. C., 1983, Cytoplasmic polyhedrosis viruses, in: The Viruses, The Reoviridae (H. Fraenkel-Conrat, R. R. Wagner, and B. Roizman, eds.), p. 425, Plenum Press, New York.

48a. Kelly, D. C., and Bud, H. M., 1978, Densonucleosis virus DNA: Analysis of fine structure by electron microscopy and agarose gel electrophoresis, J. Gen. Virol. 40:33.

48b. Tijssen, P., and Kurstak, E., 1981, Biochemical, biophysical, and biological properties of densonucleosis virus (parvovirus). III. Common sequences of structural proteins, I. Virol. 37:17-23.

48c. Matthews, R. E. F., 1982, Classification and nomenclature of viruses, Intervirology 17:74.

49a. Teninges, D., 1979, Protein and RNA composition of the structural components of Drosophila X virus, J. Gen. Virol. 45:641.

49b. Teninges, D., Ohanessian, A., Richard-Molard, C., and Contamine, D., 1979, Isolation and biological properties of Drosophila X virus, J. Gen. Virol. 42:241.

49c. Scott, M. P., Fostel, J. M., and Pardue, M. L., 1980, A new type of virus from cultured Drosophila cells: Characterization and use in studies of the heat-shock response, Cell 22:929.

50. Tignor, G. H., Murphy, F. A., Clark, H. F., Shope, R. E., Madore, P., Bauer, S. P., Buckley, S. M., and Meredith, D. C., 1977, Duvenhage virus: Morphological, biochemical, histopathological and antigenic relationships to the rabies serogroup, $J$. Gen. Virol. 37:595.

51. Rosenwirth, B., and Eggers, H. J., 1978, Structure and replication of echovirus type 
12. 1. Analysis of the polypeptides and RNA of echovirus 12 particles, Eur. J. Biochem. 92:53.

52. Todd, D., and McNulty, M. S., 1978, Biochemical studies on a virus associated with egg drop syndrome 1976, I. Gen. Virol. 40:63.

53b. Olberding, K. P., and Frost, J. W., 1975, Electron microscopical observations of the structure of the virus of viral haemorrhagic septicaemia (VHS) of rainbow trout (Salmo gairdneri), J. Gen. Virol. 27:305.

54. Merregaert, J., van Emmelo, J., Devos, R., Porter, Al., Fellner, P., and Fiers, W., 1978, The 3'-terminal nucleotide sequence of encephalomyocarditis virus RNA, Eur. I. Biochem. 82:55.

56. Matthews, R. E. F., 1982, The entomopoxvirinae, Intervirology 17:44.

56a. Kieff, E., Dambaugh, T., King, W., Heller, M., Cheung, A., van Santen, V., Hummel, M., Beisel, C., and Fennewald, S., 1983, Biochemistry of Epstein-Barr virus, in: The Viruses, The Herpesviruses, Vol. I (H. Fraenkel-Conrat, R. R. Wagner, and B. Roizman, eds.), p. 105, Plenum Press, New York.

56b. Henle, W., and Henle, G., 1983, Immunology of Epstein-Barr virus, in: The Viruses, The Herpesviruses, Vol. I (H. Fraenkel-Conrat, R. R. Wagner, and B. Roizman, eds.), p. 200, Plenum Press, New York.

56c. de-Thé, G., 1983, Epidemiology of Epstein-Barr virus and associated diseases in man, in: The Viruses, The Herpesviruses, Vol. I (H. Fraenkel-Conrat, R. R. Wagner, and B. Roizman, eds.), p. 25, Plenum Press, New York.

57. Zeegers, J. J. W., Van der Zeljst, B. A. M., and Horznnek, M. C., 1976, The structural proteins of equine arteritis virus, Virology 73:200.

58. O'Callaghan, D. J., Gentry, G. A., and Randall, C. C., 1983, The equine herpesviruses, in: The Viruses, The Herpesviruses, Vol. II (H. Fraenkel-Conrat, R. R. Wagner, and B. Roizman, eds.), p. 215, Plenum Press, New York.

59a. Parekh, B., Issel, C. J., and Montelaro, R. C., 1980, Equine infectious anemia virus, a putative lentivirus, contains polypeptides analogous to prototype-C oncornaviruses, Virology 107:520-525.

59b. Summers J., Jones, S. E., and Anderson, M. J., 1983, Characterisation of the agent of erythrocyte aplasia permits its classification as a human parvovirus, I. Gen. Virol. $64: 2527$.

60. Clark, H. F., Lief, F. S., Lunger, P. D., Waters, D., Leloup, P., Foelsch, D. W., and Wyler, R. W., 1979, Fer de Lance virus (FDLV): A probable paramyxovirus isolated from a reptile, $J$. Gen. Virol. 44:405-418.

61. Kiley, M. P. et al., 1983, A taxonomic home for marburg and ebolavirus, Intervirology 18:24.

62. Matthews, R. E. F., 1982, Classification and nomenclature of viruses, Intervirology 17:98.

63a. Goorha, R., and Murti, K. G., 1982, The genome of frog virus 3, an animal DNA virus, is circularly permuted and terminally redundant, Proc. Natl. Acad. Sci. USA 79:248-252.

63b. Murti, K. G., Goorha, R., and Granoff, A., 1982, Structure of frog virus 3 genome: Size and arrangement of nucleotid sequences as determined by electron microscopy, Virology 116:275-283.

64. Matthews, R. E. F., 1982, Classification and nomenclature of viruses, Intervirology 17:50.

65. Ganem, D., Greenbaum, L., and Varmus, H. E., 1982, Virion DNA of ground squirrel hepatitis virus: Structural analysis and molecular cloning, $J$. Virol. 44:374-383.

66. Koller, R., and Goulian, M., 1981, Synthesis of parvovirus $\mathrm{H}-1$ replicative form from viral DNA by DNA polymerase $\gamma$, Proc. Natl. Acad. Sci. USA 78:6206-6210.

67. Foulke, R. S., Rosato, R. R., and French, G. R., 1981, Structural polypeptides of hazara virus, $J$. Gen. Virol. 53:169-172.

68. Bosslet, K., and Sauer, G., 1978, Biological properties and physical map of the genome of a new papovavirus, HD virus, J. Virol. 25:596-607. 
69. Pocock, D. H., 1978, Effect of sulphydryl reagents on the biological activities, polypeptide composition and morphology of haemagglutinating encephalomyelitis virus, J. Gen. Virol. 40:93-101.

70. Gust, I. D., Coulepis, A. G., Feinstone, S. M., Locarnini, S. A., Moritsugu, Y., Najera, R., and Siegl, G., 1983, Taxonomic classification of hepatitis A virus, Intervirology 20:1-7.

71a. Robinson, W. S., Viruses of human hepatitis A and B, in: Comprehensive Virology, Vol. 14 (H. Fraenkel-Conrat and R. R. Wagner, eds.), pp. 471-526, Plenum Press, New York.

71b. Melnick, j. L., 1983, Class of hepatitis A virus as entero type 72 and hepatitis B as hepadnavirus Type 1, Intervirology 18:103.

72a. Galibert, F., Mandart, E., Fitoussi, F., Tiollais, P., and Charnay, P., 1979, Nucleotide sequence of the hepatitis B virus genome (subtype ayw) cloned in E. coli, Nature 281:646.

72b. Pasek, M., Goto, T., Gilbert, W., Zink, B., Schaller, H., MacKay, P., Leadbetter, Gl., and Murray, K., 1979, Hepatitis B virus genes and their expression in E. coli, Nature 282:575.

73. Fleckenstein, B., and Desrosiers, R. C., 1983, Herpesvirus saimiri and Herpesvirus ateles, in: The Viruses, The Herpesviruses, Vol. I (H. Fraenkel-Conrat, R. R. Wagner, and B. Roizman, eds.), p. 253, Plenum Press, New York.

74a. Roizman, B., 1983, The familey Herpesviridae: General description, taxonomy, and classification, in: The Viruses, The Herpesviruses, Vol. I (H. Fraenkel-Conrat, R. R. Wagner, and B. Roizman, eds.), p. 1, Plenum Press, New York.

74b. Matthews, R. E. F., 1982, Classification and nomenclature of viruses, Intervirology $17: 47$.

75. Krell, P. J., and Stoltz, D. B., 1980, Virus-like particles in ovary of an ichneumonid wasp: Purification and preliminary characterization, Virology 101:408.

76. Robert-Guroff, M., Fahey, K. A., Maeda, M., Nakao, Y., Ito, Y., and Gallo, R. C., 1982, Identification of HTLV p19 specific natural human antibodies by competition with monoclonal antibody, Virology 122:297.

77. Langridge, W. H. R., 1981, Biochemical properties of a persistent nonoccluded baculovirus isolated from Heliothis zea cells, Virology 112:770.

78a. Compans, R. W., and Choppin, P. W., 1975, Reproduction of povaviruses, in: Comprehensive Virology, Vol. 4 (H. Fraenkel-Conrat and R. R. Wagner, eds.), p. 179, Plenum Press, New York.

78b. Laver, W. G., Air, G. M., Webster, R. G., and Markoff, L. J., 1982, Amino acid sequence changes in antigenic variants of type A influenza virus N2 neuraminidase, Virology 122:450.

78c. Webster, R. G., Laver, W. G., Air, G. M., and Schild, G. C., 1982, Molecular mechanisms of variation in influenza viruses, Nature 296:115.

78d. Kaptein, J. S., and Nayak, D. P., 1982, Complete nucleotide sequence of the polymerase 3 gene of human influenza virus A/WNS/33, 7 . Virol. 42:55.

78e. Winter, G., and Fields, S., 1982, Nucleotide sequence of human influenza A/PR/8/ 34 segment 2, Nucl. Acids Res. 10:2135.

79. Matthews, R. E. F., 1982, Classification and nomenclature of viruses, Intervirology 17:56.

80. Huang, C. H., 1982, Studies of Japanese encephalitis in China, Adv. Virus Res. 27:71.

81a. Martin, J. D., Padgett, B. L., and Walker, D. L., 1983, Characterization of tissue culture-induced heterogeneity in DNAs of independent isolates of JC virus, J. Gen. Virol. 64:2271.

81b. Walker, D. L., and Padgett, B. L., 1983, Progressive multifocal leukoencephalopathy, in: Comprehensive Virology, Vol. 18 (H. Fraenkel-Conrat and R. R. Wagner, eds.), p. 161, Plenum Press, New York.

82. Siddell, S. G., 1982, Coronavirus JHM: Tryptic peptide fingerprinting of virion proteins and intracellular polypeptides, J. Gen. Virol. 62:259. 
83. Pudney, M., Newman, J. F. E., and Brown, F., 1978, Characterization of kawino virus, an entero-like virus isolated from the mosquito Mansonia uniformis (diptera: culicidael, I. Gen. Virol. 40:433.

84. Scotti, P. D., Gibbs, A. J., and Wrigley, N. G., 1976, Kelp fly virus, J. Gen. Virol. 30:1.

85. Westaway, E. G., and Shew, M., 1977, Proteins and glycoproteins specified by the flavivirus kunjin, Virology 80:309.

86. Gentsch, J., Wynne, L. R., Clewley, J. P., Shope, R. E., and Bishop, D. H. L., 1977, Formation of recombinants between snowshoe hare and la crosse bunyaviruses, $I$. Virol. 24:893.

87. Matthews, R. E. F., 1982, Classification and nomenclature of viruses, Intervirology $17: 127$.

88a. Lehmann-Grube, F., Peralta, L. M., Bruns, M., and Lohler, J., 1983, Persistent infection of mice with the lymphocytic choriomeningitis virus, in: Comprehensive Virology, Vol. 18 (H. Fraenkel-Conrat and R. R. Wagner, eds.), p. 43, Plenum Press, New York.

88b. Oldstone, M. B. A., 1979, Immune responses, immune tolerance, and viruses, in: Comprehensive Virology, Vol. 15 (H. Fraenkel-Conrat and R. R. Wagner, eds.), p. 1, Plenum Press, New York.

88c. Buchmeier, M. J., Lewicki, H. A., Tomori, O., and Johnson, K. M., 1980, Monoclonal antibodies to lymphocytic choriomeningitis virus react with pathogenic arenaviruses, Nature 288:486.

89. Matthews, R. E. F., 1982, Classification and nomenclature of viruses, Intervirology $17: 111$.

90. Nonoyama, M., 1983, The molecular biology of Marek's disease herpesvirus, in: The Viruses, The Herpesviruses, Vol. I (H. Fraenkel-Conrat, R. R. Wagner, and B. Roizman, eds.), p. 333, Plenum Press, New York.

91. Matthews, R. E. F., 1982, The mastadenoviruses, Intervirology 17:60.

92. Perez-Bercoff, R., and Gander, M., 1977. The genomic RNA of mengovirus. I. Location of the poly(C) tract, Virology 80:426.

93a. Chattopadhyay, S. K., Cloyd, M. W., Linemeyer, D. L., Lander, M. R., Rands, E., and Lowy, D. R., 1982, Cellular origin and role of mink cell focus-forming viruses in murine thymic lymphomas, Nature 295:25.

93b. Chattopadhyay, S. K., Lander, M. R., Gupta, S., Rands, E., and Lowy, D. R., 1981, Origin of mink cytopathic focus-forming (MCF) viruses: Comparison with ecotropic and xenotropic murine leukemia virus genomes, Virology 113:465.

94. Cotmore, S. F., Sturzenbecker, L. J., and Tattersall, P., 1983, The autonomous parvovirus MVM encodes two nonstructural proteins in addition to its capsid polypeptides, Virology 129:333.

95. Matthews, R. E. F., 1978, Classification and nomenclature of viruses, Intervirology 17:105.

96. Stohlman, S. A., and Lai, M. M. C., 1979, Phosphoproteins of murine hepatitis viruses, I. Virol. 22:672.

97a. Yagi, M. J., and Compans, R. W., 1977, Structural components of mouse mammary tumor virus. I. Polypeptides of the virion, Virology 76:751.

97b. Ball, J. K., Dekaban, G. A., McCarter, J. A., and Loosmore, S. M., 1983, Molecular biological characterization of a highly leukaemogenic virus isolated from the mouse. III. Identity with mouse mammary tumour virus, J. Gen. Virol. 64:2177.

98. Örvell, C., 1978, Structural polypeptides of mumps virus, J. Gen. Virol. 41:527.

99a. Matthews, R. E. F., 1982, Classification and nomenclature of viruses, Intervirology 17:117.

99b. Casals, J., and Tignor, G. H., 1979, The nairovirus genus; serological interrelationships, Intervirology 14:144.

100. Newman, J. F. E., and Brown, F., 1977, Further physicochemical characterization of nodamura virus. Evidence that the divided genome occurs in a single component, $J$. Gen. Virol. 38:83.

101. Matthews, R. E. F., 1982, The Nodaviridae, Intervirology 17:167. 
102a. Matthews, R. E. F., 1982, Nudaurelia $\beta$ viruses, Intervirology 17:135.

102b. Juckes, I. R. M., 1979, Comparison of some biophysical properties of the nudaurelia $\beta$ and $\epsilon$ viruses, J. Gen. Virol. 42:89-94.

102c. Reinganum, C., Robertson, J. S., and Tinsley, T. W., 1978, A new group of RNA viruses from insects, J. Gen. Virol. 40:195-202.

103a. Matthews, R. E. F., 1982, The Oncovirinae, Intervirology 17:125.

103b. Vogt, P. K., 1977, Genetics of RNA tumor viruses, in: Comprehensive Virology, Vol. 9 (H. Fraenkel-Conrat and R. R. Wagner, eds.), p. 341, Plenum Press, New York.

103c. Hanafusa, H., 1977, Cell transformation by RNA tumor viruses, in: Comprehensive Virology, Vol. 10 (H. Fraenkel-Conrat and R. R. Wagner, eds.), p. 401, Plenum Press, New York.

103d. Duesberg, P., 1979, Transforming genes of retroviruses, Cold Spring Harbor Symposium on Quantitative Biology 45:13.

104a. Verwoerd, D. W., Huismans, H., and Erasmus, B. J., 1979, Orbiviruses, in: Comprehensive Virology, Vol. 14 (H. Fraenkel-Conrat and R. R. Wagner, eds.), p. 285, Plenum Press, New York.

104b. Gorman, B. M., Taylor, J., and Walker, P. J., 1983, Orbiviruses, in: The Viruses The Reoviridae (H. Fraenkel-Conrat, R. R. Wagner, and B. Roizman, eds.), p. 287, Plenum Press, New York.

104c. Matthews, R. E. F., 1982, The Orbiviruses, Intervirology 17:82.

105a. Matthews, R. E. F., 1982, The Orthomyxoviridae, Intervirology 17:106.

105b. Hightower, L. E., and Bratt, M. A., 1975, Genetics of orthomyxoviruses, in: Comprehensive Virology, Vol. 9 (H. Fraenkel-Conrat and R. R. Wagner, eds.), p. 535, Plenum Press, New York.

106. Matthews, R. E. F., 1982, The orthopoxviruses, Intervirology 17:42.

107. Matthews, R. E. F., 1982, The orthoreoviruses, Intervirology 17:81.

108. Payne, C. C., 1974, The isolation and characterization of a virus from Oryctes rhinoceros, J. Gen. Virol. 25:105.

109. Matthews, R. E. F., 1982, Classification and nomenclature of viruses, Intervirology 17:62.

110a. Salzman, N. P., and Khoury, G., 1979 Reproduction of papovaviruses, in: Comprehensive Virology, Vol. 3 (H. Fraenkel-Conrat and R. R. Wagner, eds.), p. 63, Plenum Press, New York.

110b. Finch, J. T., and Crawford, L. V., 1975, Structure of small DNA-containing animal viruses, in: Comprehensive Virology, Vol. 4 (H. Fraenkel-Conrat and R. R. Wagner, eds.), p. 119, Plenum Press, New York.

110c. Matthews, R. E. F., 1982, Classification and nomenclature of viruses, Intervirology 17:62.

111a. Choppin, P. W., and Compans, R. W., 1975, Reproduction of paramyxoviruses, in: Comprehensive Virology, Vol. 4 (H. Fraenkel-Conrat and R. R. Wagner, eds.), p. 95, Plenum Press, New York.

111b. Matthews, R. E. F., 1982, Classification and nomenclature of viruses, Intervirology 17:104.

111c. Kingsbury, D. W., Bratt, M. A., Choppin, W., Hanson, R. P., Hosaka, Y., ter Muelen, V., Norrby, E., Plowright, W., Rott, R., and Wunner, W. H., 1978, Paramyxoviridae, Intervirology 10:137.

111d. Matthews, H. E. F., 1982, Classification and nomenclature of viruses, Intervirology 17:43.

112a. Rose, J. A., 1974, Parvovirus reproduction, in: Comprehensive Virology, Vol. 3 (H. Fraenkel-Conrat and R. R. Wagner, eds.), p. 1, Plenum Press, New York.

112b. Bachmann, P. A., Hoggan, M. D., Kurstak, E., Melnikc, J. L., Pereira, H. G., Tattersall, P., and Vago, C., 1979, Parvoviridae: Second report, Intervirology 11:248.

112c. Matthews, H. E. F., 1982, Classification and nomenclature of viruses, Intervirology 17:72.

113. Matthews, H. E. F. 1982, Classification and nomenclature of viruses, Intervirology $17: 100$. 
114. Matthews, H. E. F., 1982, Classification and nomenclature of viruses, Intervirology 17:116.

115a. Crowell, R. L., and Landau, B. J., 1983, Receptors in the initiation of picornavirus infections, in: Comprehensive Virology, Vol. 18 (H. Fraenkel-Conrat and R. R. Wagner, eds.l, p. 1, Plenum Press, New York.

115b. Rueckert, R. R., 1975, On the structure and morphogenesis of picornaviruses, in: Comprehensive Virology, Vol. 5 (H. Fraenkel-Conrat and R. R. Wagner, eds.), p. 31, Plenum Press, New York.

115c. Cooper, P. D., 1977, Genetics of picornaviruses, in: Comprehensive Virology, Vol. 9 (H. Fraenkel-Conrat and R. R. Wagner, eds.), p. 133, Plenum Press, New York.

115d. Cooper, P. D., Agol, V. I., Bachrach, H. L., Brown, F., Ghendon, Y., Gibbs, A. J., Gillespie, J. H., Lonberg-Holm, K., Mandel, B., Melnick, J. L., Mohanty, S. B., Povey, R. C., Rueckert, R. R., Schaffer, F. L., and Tyrrell, D. A. J., 1978, Picornaviridae: Second report, Intervirology 10:165.

115e. Matthews, R. E. F., 1982, Classification and nomenclature of viruses, Intervirology 17:129.

116. Matthews, R. E. F., 1982, Classification and nomenclature of viruses, Intervirology 17:105.

117. Kitamura, N., Semler, B. L., Rothberg, P. G., Larsen, G. R., Adler, C. J., Dorner, A. J., Emini, E. A., Hanecak, R., Lee, J. J., van der Werf, S., Anderson, C. W., and Wimmer, E., 1981, Primary structure, gene organization and polypeptide expression of poliovirus RNA, Nature 291:547.

117a. Stoltz et al., 1984, Polydnaviridae: proposed family of insect viruses with segmented double-stranded circular DNA genome, Intervirology 21:1.

118a. Eckhart, W., 1977, Genetics of polyoma virus and simian virus 40, in: Comprehensive Virology, Vol. 9 (H. Fraenkel-Conrat and R. R. Wagner, eds.), p. 1, Plenum Press, New York.

118b. Matthews, R. E. F., 1982, Classification and nomenclature of viruses, Intervirology $17: 62$.

119a. Moss, B., 1974, Reproduction of poxviruses, in: Comprehensive Virology, Vol. 3 (H. Fraenkel-Conrat and R. R. Wagner, eds.), p. 405, Plenum Press, New York.

119b. Gafford, L. G., Mitchell, E. B., Jr., and Randall, C. C., 1978, Sedimentation characteristics and molecular weights of three poxvirus DNAs, Virology 89:229.

119c. Matthews, R. E. F., 1982, Classification and nomenclature of viruses, Intervirology $17: 42$.

120. Matthews, R. E. F., 1982, Classification and nomenclature of viruses, Intervirology 17:57.

121a. Sharpe, A. H., and Fields, B. N., 1983, Pathogenesis of reovirus infection, in: The Viruses, The Reoviridae (H. Fraenkel-Conrat, R. R. Wagner, and B. Roizman, eds.), p. 229, Plenum Press, New York.

121b. Joklik, W. K., 1983, The Viruses, The Reoviridae (H. Fraenkel-Conrat and R. R. Wagner, eds.), p. 1, Plenum Press, New York.

121c. Joklik, W. K., 1983, The reovirus particle, in: The Viruses, The Reoviridae (H. Fraenkel-Conrat and R. R. Wagner, eds.), p. 9, Plenum Press, New York.

121d. Shatkin, A. J., and Kozak, M., 1983, Biochemical aspects of reovirus transcription and translation, in: The Viruses, The Reoviridae (H. Fraenkel-Conrat and R. R. Wagner, eds.), p. 79, Plenum Press, New York.

121 e. Matthews, R. E. F., 1982, Classification and nomenclature of viruses, Intervirology 17:81.

122. Lambert, D. M., Pons, M. W., Mbuy, G. N., and Hasler, K. D., 1980, Nucleic acids of respiratory syncytial virus, $J$. Virol. 36:837.

123a. Matthews, R. E. H., 1982, Classification and nomenclature of viruses, Intervirology 17:124.

123b. Duesberg, P. H., 1983, Retroviral transforming genes in normal cells? Nature 304:219.

124a. Wagner, R. R., 1975, Reproduction of rhabdoviruses, in: Comprehensive Virology, Vol. 4 (H. Fraenkel-Conrat and R. R. Wagner, eds.), p. 1, Plenum Press, New York. 
124b. Pringle, C. R., 1977, Genetics of rhabdoviruses, in: Comprehensive Virology, Vol. 9 (H. Fraenkel-Conrat and R. R. Wagner, eds.), p. 239, Plenum Press, New York.

124c. Matthews, R. E. F., 1982, Classification and nomenclature of viruses, Intervirology 17:109

125. Matthews, R. E. F., 1982, Classification and nomenclature of viruses, Intervirology $17: 130$.

126a. Holmes, I. H., 1983, Rotaviruses, in: The Viruses, The Reoviridae (H. Fraenkel-Conrat, R. R. Wagner, and B. Roizman, eds.|, p. 359, Plenum Press, New York.

126b. Matthews, R. E. F., 1982, Classification and nomenclature of viruses, Intervirology 17:84.

127a. Keith, J., and Fraenkel-Conrat, H., 1975, Identification of the 5' end of rous sarcoma virus RNA, Proc. Natl. Acad. Sci. USA 72:3347.

127b. Hackett, P. B., Swanstrom, R., Varmus, H. E., and Bishop, J. M., 1982, The leader sequence of the subgenomic mRNA's of rous sarcoma virus is approximately 390 nucleotides, $J$. Virol. 41:527.

128. Van Alstyne, D., Drystal, G., Kettyls, G. D., and Bohn, E. M., 1981, The purification of rubella virus (RV) and determination of its polypeptide composition, Virology 108:491.

129a. Marsh, R. F., Malone, T. G., and Lancaster, R. P., 1978, Evidence for an essential DNA component in the scrapie agent, Nature 275:147.

129b. Cho, H. J., 1979, Requirement of a protein component for scrapie infection, Intervirology 14:213.

130. Monroe, S. S., Ou J-H., Rice, C. M., Schlesinger, S., Strauss, E. G., and Strauss, J. H., 1982, Sequence analysis of cDNAs derived from the RNA of sindbis virions and of defective interfering particles, $J$. Virol. 41:153.

131a. Schultz, A. M., Ruscetti, S. K., Scolnick, E. M., and Oroszlan, S., 1980, The env-gene of the spleen focus-forming virus lacks expression of p15(E) determinants, Virology 107:537.

131b. Linemeyer, D. L., Ruscetti, S. K., Scolnick, E. M., Evans, L. H., and Duesberg, P. H., 1981, Biological activity of the spleen focus-forming virus is encoded by a molecularly cloned subgenomic fragment of spleen focus-forming virus DNA, Proc. Natl. Acad. Sci. USA 78:1401.

132. O'Rear, J. J., and Temin, H. M., 1981, Mapping of alterations of noninfectious proviruses of spleen necrosis virus, I. Virol. 39:138.

133. Matthews, R. E. F., 1982, Classification and nomencalture of viruses, Intervirology 17:126.

134. ter Meulen, V., Stephenson, J. R., and Kreth, H. W., 1974, Subacute sclerosing panencephalitis, in: Comprehensive Virology, Vol. 18 (H. Fraenkel-Conrat and R. R. Wagner, eds.), p. 105, Plenum Press, New York.

135. Matthews, R. E. F., 1982, Classification and nomenclature of viruses, Intervirology 17:44.

136. Fiers, W., Contreras, R., Haegeman, G., Rogiers, R., Van de Voorde, A., Van Heuverswyn, H., Van Herreweghe, V., Volckaert, G., and Ysebaert, M., 1978, Complete nucleotide sequence of SV40 DNA, Nature 273:113.

137a. Matthews, R. E. F., 1982, Classification and nomenclature of viruses, Intervirology 17:97.

137b. Pfefferkorn, E. R., and Shapiro, D., 1974, Reproduction of togaviruses, in: Comprehensive Virology, Vol. 2 (H. Fraenkel-Conrat and R. R. Wagner, eds.), p. 171, Plenum Press, New York.

137c. Pfefferkorn, E. R., 1977, Genetics of togaviruses, in: Comprehensive Virology, Vol. 9 (H. Fraenkel-Conrat and R. R. Wagner, eds.), p. 209, Plenum Press, New York.

138a. Matthews, R. E. F., 1982, Classification and nomenclature of viruses, Intervirology 17:117.

138b. Hewlett, M. J., Pettersson, R. F., and Baltimore, D., 1977, Circular forms of uukuniemi virion RNA: An electron microscopic study, J. Virol. 21:1085. 
139a. Belle Isle, H., Venkatesan, S., and Moss, B., 1981, Cell-free translation of early and late mRNAs selected by hybridization to cloned DNA fragments derived from the left 14-million to 72-million daltons of the vaccinia virus genome, Virology 112:306.

139b. Wittek, R., Barbosa, E., Cooper, J. A., Garon, C. F., Chan, H., and Moss, B., 1980, Inverted terminal repetition in vaccinia virus DNA encodes early mRNAs, Nature 285:21.

140. Hyman, R. W., 1983, Molecular biology of varicella-zoster virus, in: The Viruses, The Herpesviruses, Vol. II (H. Fraenkel-Conrat, R. R. Wagner, and B. Roizman, eds.), p. 115, Plenum Press, New York.

141. Matthews, R. E. F., 1982, Classification and nomenclature of viruses, Intervirology 17:110.

142. Harris, J. D., Scott, J. V., Traynor, B., Brahic, M., Stowring, L., Ventura, P., Haase, A. T., and Peluso, R., 1981, Visna virus DNA: Discovery of a novel gapped structure, Virology 113:573.

143a. DeJean, A., Vitvitski, L., Brechot, C., Trepo, C., Tiollais, P., and Charnay, P., 1982, Presence and state of woodchuck hepatitis virus DNA in liver and serum of woodchucks: Further analogies with human hepatitis B virus, Virology 121:195.

143b. Galibert, F., Chen, T. N., and Mandart, E., 1982, Nucleotide sequence of a cloned woodchuck hepatitis virus genome: Comparison with the hepatitis B virus sequence, J. Virol. 41:51. 